\title{
Core Average (MOX/LEU) Nuclide Ratios for Sequoyah and Browns Ferry Reactors (Nonproprietary)
}

\section{April 2013}

Prepared by

Harold J. Smith

Bruce B. Bevard

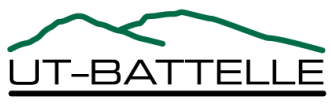


This report was prepared as an account of work sponsored by an agency of the United States Government. Neither the United States Government nor any agency thereof, nor any of their employees, makes any warranty, express or implied, or assumes any legal liability or responsibility for the accuracy, completeness, or usefulness of any information, apparatus, product, or process disclosed, or represents that its use would not infringe privately owned rights. Reference herein to any specific commercial product, process, or service by trade name, trademark, manufacturer, or otherwise, does not necessarily constitute or imply its endorsement, recommendation, or favoring by the United States Government or any agency thereof. The views and opinions of authors expressed herein do not necessarily state or reflect those of the United States Government or any agency thereof. 
Harold J. Smith

Bruce B. Bevard

Date Published: April 2013

\author{
Prepared by \\ OAK RIDGE NATIONAL LABORATORY \\ Oak Ridge, Tennessee 37831-6283 \\ managed by \\ UT-BATTELLE, LLC \\ for the \\ U.S. DEPARTMENT OF ENERGY \\ under contract DE-AC05-00OR22725
}





\section{TABLE OF CONTENTS}

Page

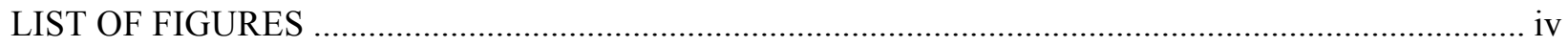

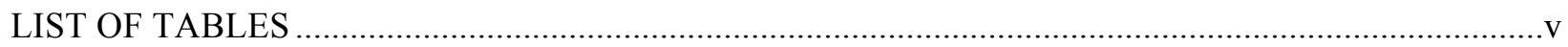

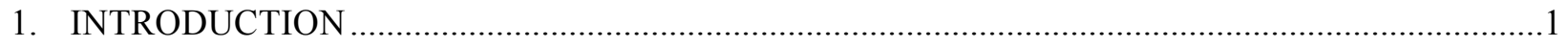

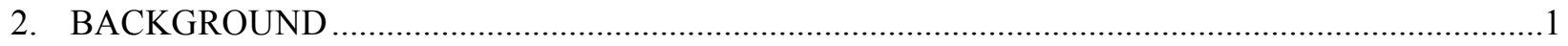

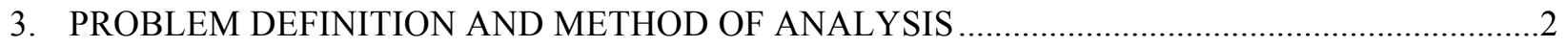

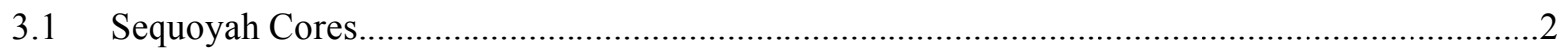

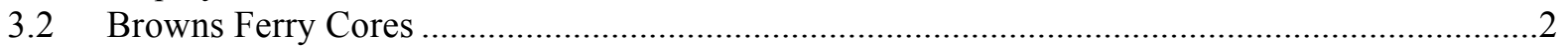

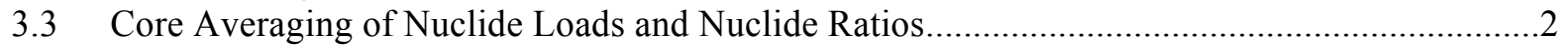

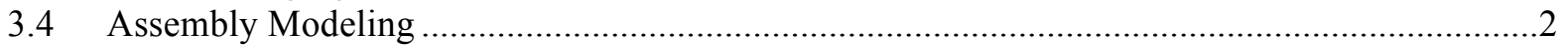

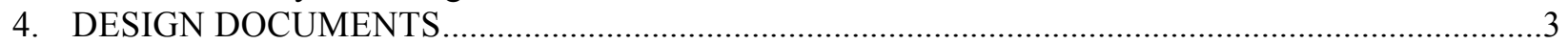

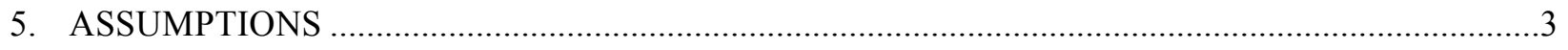

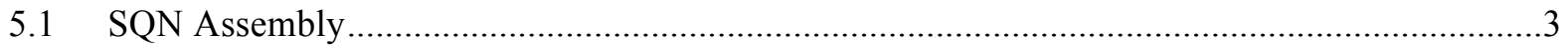

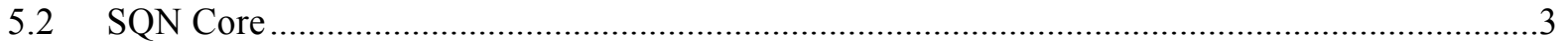

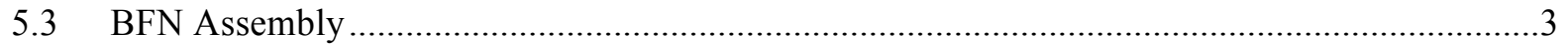

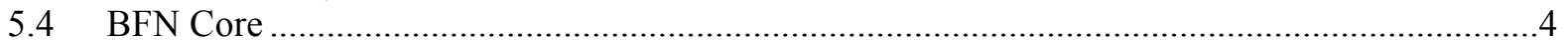

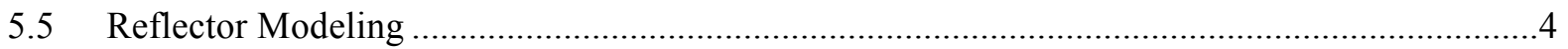

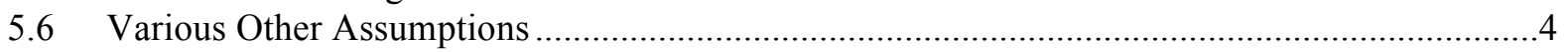

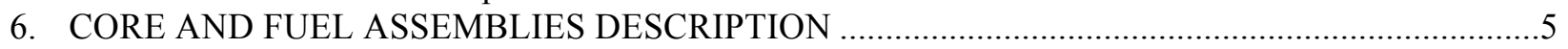

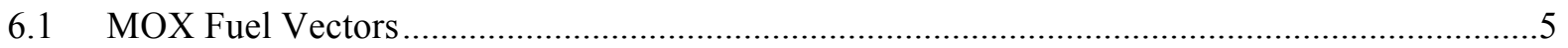

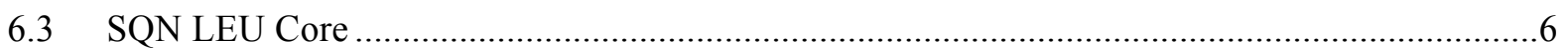

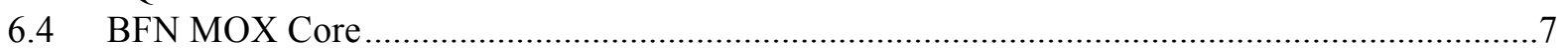

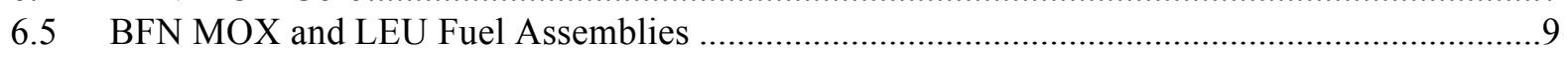

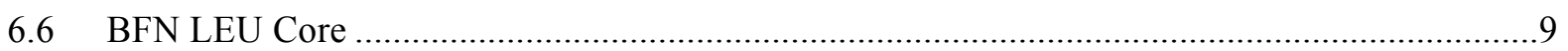

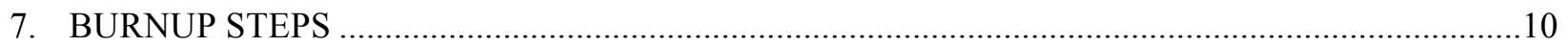

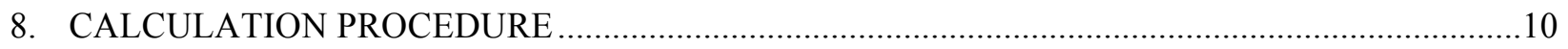

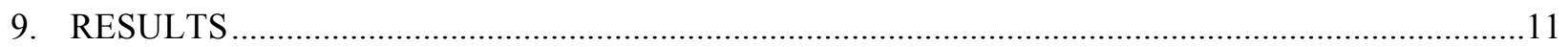

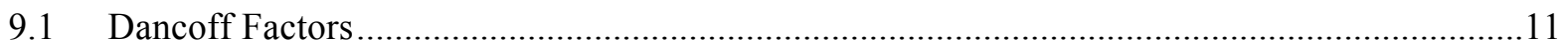

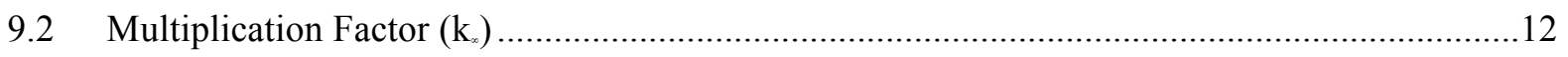

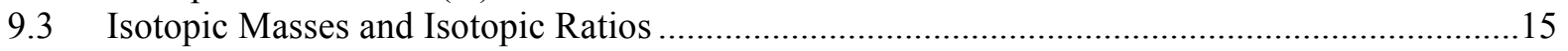

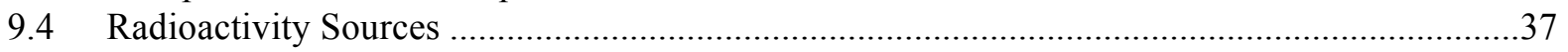

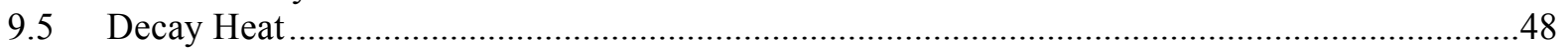

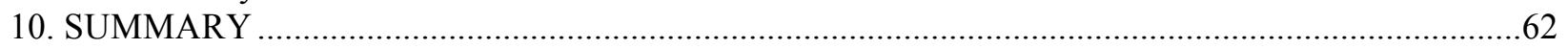

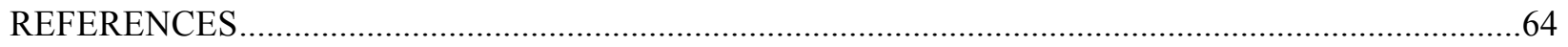




\section{LIST OF FIGURES}

1. SQN equilibrium cycle MOX core assembly identification map. …................................................5

2. SQN equilibrium LEU core assembly identification map. …........................................................

3. BFN MOX equilibrium core assay/enrichment and EOC burnup distribution....................................

4. BFN equilibrium EOC burnup distribution in LEU core (GWd/MTHM) .....................................

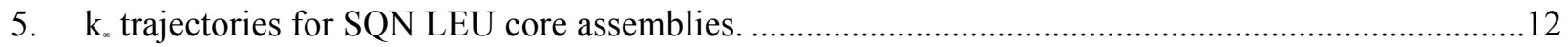

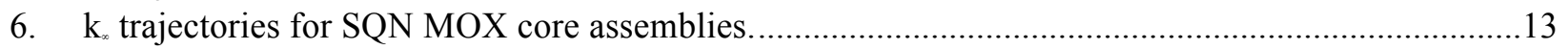

7. $\mathrm{k}_{x}$ trajectories for BFN LEU core assemblies - dominant zone.....................................................13

8. $\mathrm{k}_{x}$ trajectories for BFN LEU core assemblies - vanished zone.......................................................14

9. $\mathrm{k}_{\star}$ trajectories for BFN MOX core assemblies - dominant zone. ..................................................14

10. $\mathrm{k}_{\star}$ trajectories for BFN MOX core assemblies - vanished zone. …................................................15

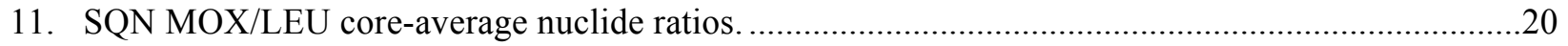

12. SQN \% differences of MOX/LEU nuclide ratios from DOE/EIS-0283 values.................................25

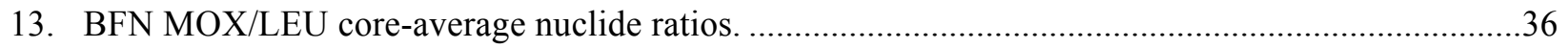

14. BFN \% differences of MOX/LEU nuclide ratios from DOE/EIS-0283 PWR values. .......................37

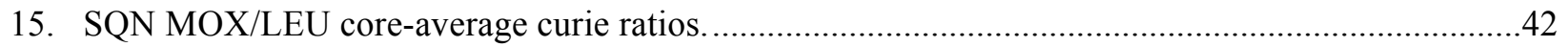

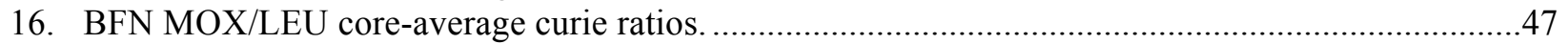

17. Comparison of assembly decay heat trajectories in the SQN equilibrium LEU core 100 years.

18. Comparison of assembly decay heat trajectories in the SQN equilibrium MOX core 100 years, part A.

19. Comparison of assembly decay heat trajectories in the SQN equilibrium MOX core 100 years, part B.

20. Comparison of decay heat in SQN equilibrium MOX and LEU cores -1 year...............................52

21. Comparison of "A" assembly decay heat trajectories in the BFN equilibrium LEU core 100 years.

22. Comparison of "B" assembly decay heat trajectories in the BFN equilibrium LEU core 100 years

23. Comparison of "C" assembly decay heat trajectories in the BFN equilibrium LEU core 100 years.

24. Comparison of "A" assembly decay heat trajectories in the BFN equilibrium MOX core 100 years.

25. Comparison of "B" assembly decay heat trajectories in the BFN equilibrium MOX core -

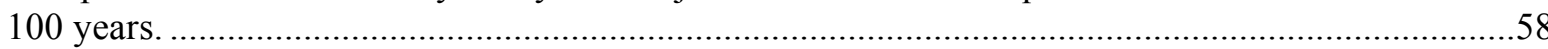

26. Comparison of "C" assembly decay heat trajectories in the BFN equilibrium MOX core 100 years.

27. Comparison of "D" assembly decay heat trajectories in the BFN equilibrium MOX core100 years.

28. Comparison of decay heat in BFN equilibrium MOX and LEU cores -1 year. 


\section{LIST OF TABLES}

Table

Page

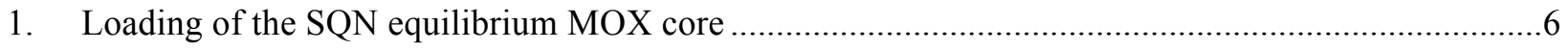

2. Loading of the SQN equilibrium LEU core

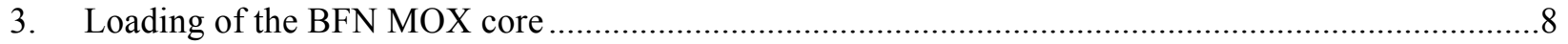

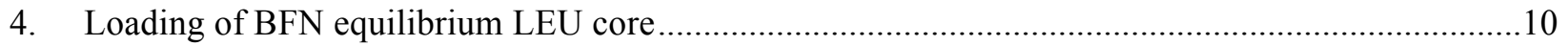

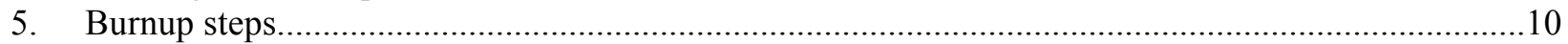

6. Summary of nuclide concentrations for the SQN equilibrium MOX and LEU cores .......................15

7. Comparison of SQN nuclide concentration ratios to Reference [3] ..............................................21

8. Summary of nuclide concentrations for the BFN equilibrium MOX and LEU cores ........................27

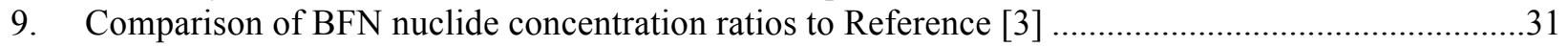

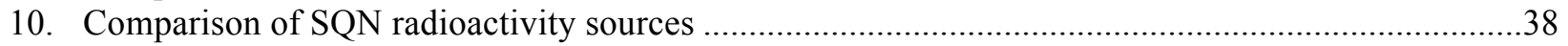

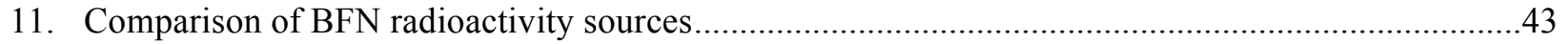

12. Decay heat (W/MTHM) for the fuel assemblies in the SQN LEU core ...........................................49

13. Decay heat (W/MTHM) for the fuel assemblies in the SQN MOX core - part A ...........................50

14. Decay heat (W/MTHM) for the fuel assemblies in the SQN MOX core - part B ...........................51

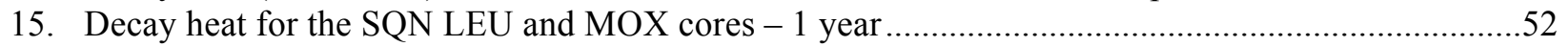

16. Decay heat (W/MTHM) for the fuel "A" assembly in the BFN LEU core .....................................54

17. Decay heat (W/MTHM) for the fuel "B" assembly in the BFN LEU core ......................................55

18. Decay heat (W/MTHM) for the fuel "C" assembly in the BFN LEU core ......................................56

19. Decay heat (W/MTHM) for the fuel "A" assembly in the BFN MOX core.......................................57

20. Decay heat (W/MTHM) for the fuel "B" assembly in the BFN MOX core .....................................58

21. Decay heat (W/MTHM) for the fuel "C" assembly in the BFN MOX core .....................................59

22. Decay heat (W/MTHM) for the fuel "D" assembly in the BFN MOX core .......................................60

23. Decay heat for the BFN equilibrium cores -1 year ..................................................................61 



\section{INTRODUCTION}

Oak Ridge National Laboratory (ORNL) was directed by the Department of Energy NN-26 to develop and run SCALE/TRITON [1] models for the generation of core-average nuclide inventories, in a MOX core $(61 \% \mathrm{LEU}+39 \% \mathrm{MOX})$ and a LEU core with assembly burnups as high as $\sim 60 \mathrm{GWd} / \mathrm{MTU}$, for a Sequoyah pressurized water reactor (PWR) and a Browns Ferry boiling water reactor (BWR). This study compares the nuclide ratios of a representative MOX core to a representative LEU core for both the Sequoyah (SQN) and Browns Ferry (BFN) reactors. Previous work [2, 3], supporting earlier Environmental Impact Statement (EIS) reports, considered only a PWR assembly.

The previous work $[2,3]$ determined the nuclide ratios assuming a LEU assembly with a uniform loading of $4.37 \mathrm{wt} \%$ U-235 enrichment fuel rods and a MOX assembly with a uniform loading of $4.37 \mathrm{wt} \%$ (PuAm) assay fuel rods. The intention in the current study is to utilize the mechanical design of the actual SQN and BFN assemblies with current proposed core enrichment loadings (LEU and MOX) at SQN and BFN. This deviates from the philosophy of the original studies that compared identical but hypothetical enrichment loadings and is deemed to be more appropriate in light of the current status of program development.

In the current work, the same weapons-grade (Pu-Am) vector is used in the MOX fuel for both SQN and BFN. Since the amount of $(\mathrm{Pu}-\mathrm{Am})$ in a fuel rod is not, strictly speaking, an enrichment, it is referred to in this report as an "assay."

The W17 HTP assembly configuration [4] is used in this work for the modeling of the SQN reactors. The assembly characteristics are close to but not identical to the values used for the previous calculations [2].

The SQN data reflects three different (Pu-Am) assays in the W17 HTP MOX assembly. The nominal average (Pu-Am) assay for this assembly is $4.35 \mathrm{wt} \%$ in the current study compared to the $4.37 \mathrm{wt} \%$ assay of the earlier study.

For the BFN reactors, the analysis uses the ATRIUM $10 \times 10$ fuel assembly design. This assembly is quite heterogeneous with respect to the number of $(\mathrm{LEU}+\mathrm{Gd})$ rods, the locations of these rods in the assembly, or the $\mathrm{Gd}_{2} \mathrm{O}_{3}$ content in (LEU+Gd) fuel used in these rods. A MOX assembly using only four (Pu-Am) assays was selected as the candidate assembly design for the BFN reactors.

\section{BACKGROUND}

The original work [2] was developed in 1995 using simple models and computational capabilities as available at that time. Since then, there have been substantial improvements in code capabilities and nuclear data. The computational analysis in the current report has been performed using only the SCALE nuclear analysis code system [1]. The current modeling and simulation capabilities in SCALE allow a full two-dimensional (2D) representation of the fuel assembly, an improved cross section self-shielding treatment, and the use of individual fuel rod Dancoff factors, whereas the models used in Reference [2] used a simplified one-dimensional (1D) representation of the fuel assembly and older versions of nuclear data. The current SCALE computational capabilities include an improved 238-group ENDF-B/VII cross section library that is based on the most recent cross section data evaluations, whereas a 27-group cross section library was used in Reference [2], which was based mostly on ENDF-B/IV cross sections with some ENDF-B/V cross sections data included. 


\section{PROBLEM DEFINITION AND METHOD OF ANALYSIS}

This section presents a description of the problem and an overview of the analysis process.

To obtain the nuclide ratios required for the EIS report, it was necessary to determine the average concentration of all required nuclides in each of the MOX and LEU equilibrium cores for each reactor type (SQN, BFN). The number and type of assemblies and the distribution of assembly burnups in the equilibrium cores were determined by fuel management studies [5, 6, 7 and 8].

\subsection{Sequoyah Cores}

The SQN equilibrium MOX core consists of 193 fuel assemblies that are a mix of LEU and MOX fuel assemblies. The weighted average burnup for the SQN MOX core is $37.10 \mathrm{GWd} / \mathrm{MTHM}$.

The SQN equilibrium LEU core consists of $100 \%$ LEU fuel assemblies with three enrichments. The weighted average burnup for the SQN LEU core is $38.06 \mathrm{GWd} / \mathrm{MTHM}$.

\subsection{Browns Ferry Cores}

The BFN equilibrium MOX core consists of 764 fuel assemblies that are a mix of LEU and MOX fuel assemblies. The average burnup for the BFN MOX core is $33.08 \mathrm{GWd} / \mathrm{MTHM}$.

The BFN equilibrium LEU core consists of $100 \%$ LEU fuel assemblies. The weighted average burnup for the BFN LEU core is $35.46 \mathrm{GWd} / \mathrm{MTHM}$.

\subsection{Core Averaging of Nuclide Loads and Nuclide Ratios}

The core (Pu-Am) assay/U-235 enrichment and burnup loading maps were obtained from the fuel management studies that defined the equilibrium MOX and LEU cores for each reactor type. Using these maps, it is possible to determine the number of each type of assembly in each type of core. As noted, each type of assembly can be characterized into two or three burnup classes (low, medium, high). The average burnup for each class was determined for each assembly type. Thus, it could be determined what fraction of the core loading is represented by each burnup class of each assembly. Depletion simulations were performed for each assembly type model to deplete to $\sim 60 \mathrm{GWd} / \mathrm{MTHM}$, thus covering the entire range of assembly burnups in the core. These data served to interpolate for calculating the nuclide concentrations that correspond to the average burnups of the burnup classes for each assembly type.

The nuclide concentrations thus determined were summed and weighted by the core fraction of that burnup class for each assembly type to produce a core-average value of the nuclide concentrations. The nuclide concentration ratios were determined from these weighted sums for the MOX and LEU cores. The core loads were determined by multiplying the concentrations (g/MTHM) by the MTHM per assembly and then by the number of assemblies in the core.

\subsection{Assembly Modeling}

Previous studies $[9,10]$ have highlighted the need to calculate fuel rod Dancoff factors independently of the standard TRITON/MIPLIB process for highly heterogeneous BWR fuel assemblies. The SCALE module MCDANCOFF was employed to calculate Dancoff factors for every fuel rod in the assembly. Dancoff factor maps were therefore generated for the ATRIUM 10 dominant and vanished lattices (short rods replaced by moderator) at void concentrations of $0 \%, 40 \%$, and $80 \%$. Using a typical void profile, an 
average void of $25 \%$ was determined for the dominant lattice and an average void of $70 \%$ was determined for the vanished lattice. The void profiles are almost identical for the BFN MOX and LEU assemblies. Hence, only the two void levels described above were used. These void concentrations were used in the depletion calculations of each type of lattice. This work was carried out using SCALE version 6.1, which permits the use of MCDANCOFF-calculated Dancoff factors.

This project used a format for TRITON input files that is based on naming fuels, used in the models, by their array positions, thus making the descriptions clear and explicit. This strategy leads to relatively large input files (>1000 lines) but promotes consistency and clear understanding of the model.

\section{DESIGN DOCUMENTS}

References [4 to 8] are the base documents used to develop the MOX and LEU models for this study. Reference [4] was not used directly. However, by means of a private communication with AREVA, the physical details of the W17 HTP assembly were confirmed. Reference [5] reports the results of a fuel cycle study to produce an equilibrium MOX core in the Sequoyah reactors; the assembly assay and enrichment loadings and end of cycle (EOC) burnup distribution were taken from this reference.

Reference [6] reports a fuel cycle study of an all LEU core using commercial-grade uranium (CGU) in the Sequoyah reactors; the assembly enrichments and EOC burnup distribution for the SQN equilibrium LEU core were taken from this reference.

Reference [7] describes a study of both an equilibrium LEU core and two variations of a MOX core in a Browns Ferry reactor. The information for the BFN equilibrium LEU core was taken from this document. The MOX cores in Reference [7] provided preliminary information, which led to a second MOX core study reported in Reference [8]. The core reported in this document [8] considered one variant of the core in which the MOX fuel assembly was re-designed to utilize only four MOX fuel rod assays. It is this core from Reference [8] that has been modeled in the current study and reported in the current document.

\section{ASSUMPTIONS}

\subsection{SQN Assembly}

The conceptual fuel management design studies $[5,6]$ used many combinations for the number and locations of ( $\mathrm{LEU}+\mathrm{Gd})$ rods and the $\mathrm{Gd}_{2} \mathrm{O}_{3}$ concentration in these rods. A decision was taken to model the SQN MOX and LEU assemblies with $20 \mathrm{LEU}+\mathrm{Gd}$ rods.

\subsection{SQN Core}

For a given assembly type and a given burnup class for that assembly, nuclide concentrations were determined at a burnup value that corresponded to the average burnup of the burnup class. These nuclide concentrations were used for all burnups in the considered burnup class for that assembly.

\subsection{BFN Assembly}

The conceptual fuel management design studies [7, 8] provided explicit assembly loadings for the MOX and LEU assemblies in each core. The BFN MOX assemblies are well represented by a single dominant lattice zone and a single vanished lattice zone. The average enrichments in each lattice are slightly different as a result of the enrichment changes due to vanishing rods. Plenum lattices (transition zones where the short rods disappear) were not modeled. The BFN LEU assemblies had three or four lattices (in 
addition to the natural uranium blankets). The major lattice in each of the dominant and vanished lattices was used to represent the entire assembly. The differences in the average enrichments were very small.

\subsection{BFN Core}

For a given assembly type and a given burnup class for that assembly, nuclide concentrations were determined at a burnup value that corresponded to the average burnup of the burnup class. These nuclide concentrations were used for all burnups in the considered burnup class for that assembly.

\subsection{Reflector Modeling}

These calculations consider infinite lattices of the specified fuel assembly. There is no reflector modeling included in the models used in this study.

\subsection{Various Other Assumptions}

During model development, various other assumptions were made. The assumptions are listed here with a short description/justification.

1. Lattice geometry - all data obtained from References [4 to 8] plus comments received on the Data Call.

2. All models are 2D.

3. Cross-section libraries-SCALE's ENDF/B-VII 238-group library was the basis for all calculations. SCALE/TRITON's "parm=weight" option was used to collapse the 238-group master library to a 49-group problem-dependent library at depletion time $\mathrm{T}=0$.

4. Resonance processing - all calculations use CENTRM/PMC [11] for resonance self-shielding. Nongadolinium-bearing fuel rods use a lattice cell treatment in CENTRM. The gadolinium-bearing fuel rods use a multiregion treatment; the fuel region in the fuel rod is represented as five equal-area rings for the purpose of better modeling the radial depletion of gadolinium as a function of the radial distance from the rod center. Internal studies have found that five equal-area rings provide reasonable results while reducing the required computer time.

5. Dancoff factors for fuel rods were calculated for BWR assemblies, using the SCALE/MCDANCOFF module for 0,40 , and $80 \%$ void concentrations.

6. Depletion - all different fuel mixtures were depleted individually. Non-gadolinium-bearing rods were depleted by rod power, while gadolinium-bearing rods were depleted by flux. To reduce computing time, the SCALE/TRITON “assign" function was used to group rods for resonance processing purposes. Tests have shown that the use of this technique results in less than $\sim 10 \mathrm{pcm}$ of bias in the multiplication factor $\left(\mathrm{k}_{\mathrm{inf}}\right)$ compared to a "CENTRM for every rod" approach. The option addnux $=4$ was used in all cases. The use of this option ensures that the cross sections for those isotopes ( 400) for which data exist in the 238-group ENDF/B-VII transport library are updated at each depletion step for use in the depletion solver.

7. Transport model-All fuel mixtures and structural material use $\mathrm{P}_{1}$ scattering, while all moderator/coolant mixtures use $\mathrm{P}_{2}$ scattering. Convergence criteria for the eigenvalue were set at $10^{-5}$. Coarse-mesh finite-difference acceleration was used on the global grid.

8. History calculations - BWR calculations were run with $25 \%$ average void in the dominant zone and $70 \%$ void in the vanished zone.

9. No control rods were defined for the PWR cases. All BWR cases were run with control blades removed. 


\section{CORE AND FUEL ASSEMBLIES DESCRIPTION}

\subsection{MOX Fuel Vectors}

The MOX fuel used for both the SQN and BFN models consists of weapons-grade (WG) plutoniumamericium mixed with depleted uranium. The $\mathrm{Pu}-\mathrm{Am}$ vector is proprietary but was based on fuel used in the Catawba reactor.

\subsection{SQN MOX Core}

The SQN MOX core consists of both MOX and LEU fuel assemblies. Figure 1 presents a $1 / 4$ core map for the SQN MOX equilibrium core, showing the assembly identifiers.

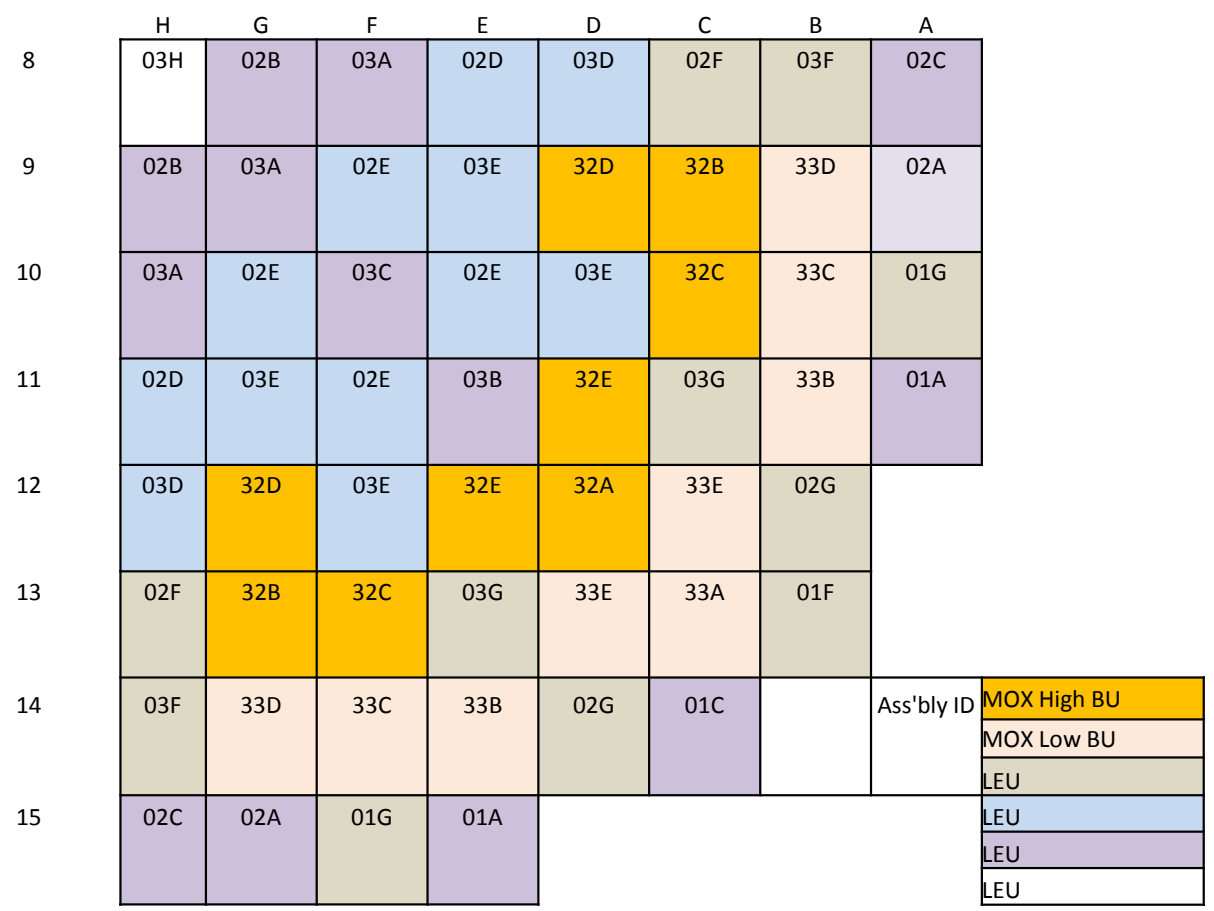

Figure 1. SQN equilibrium cycle MOX core assembly identification map.

Table 1 summarizes the categories of the assemblies by assembly type, burnup class, average burnups in each burnup class, and fraction of each assembly category in the MOX core. The color coding of the MOX assemblies represents the two burnup classes. The color coding of the LEU assemblies represents the various U-235 enrichments. 
Table 1. Loading of the SQN equilibrium MOX core

\begin{tabular}{||c||c||c||c||}
\hline $\begin{array}{c}\text { Assembly } \\
\text { type }\end{array}$ & $\begin{array}{c}\text { Burnup } \\
\text { class }\end{array}$ & $\begin{array}{c}\text { Number of } \\
\text { assemblies in core }\end{array}$ & $\begin{array}{c}\text { Fraction } \\
\text { in core }\end{array}$ \\
\hline \hline MOX & 1 & 36 & 0.1865 \\
\hline \hline & 2 & 36 & 0.1865 \\
\hline \hline LEU & 1 & 1 & 0.0052 \\
\hline \hline & 1 & 12 & 0.0622 \\
\hline \hline & 2 & 16 & 0.0829 \\
\hline \hline & 3 & 12 & 0.0622 \\
\hline \hline & 1 & 16 & 0.0829 \\
\hline \hline & 3 & 0 & 0.0829 \\
\hline \hline & 1 & 16 & 0.0622 \\
\hline \hline & 2 & 12 & 0.0415 \\
\hline \hline Total & 3 & 8 & 0.1451 \\
\hline
\end{tabular}

\subsection{SQN LEU Core}

Figure 2 presents a $1 / 4$ core map of the SQN equilibrium LEU core showing assembly identification numbers. Assemblies have been highlighted in different colors to clarify their location and distribution in the core.

The assemblies with higher U-235 enrichments are each categorized in three burnup classes. 


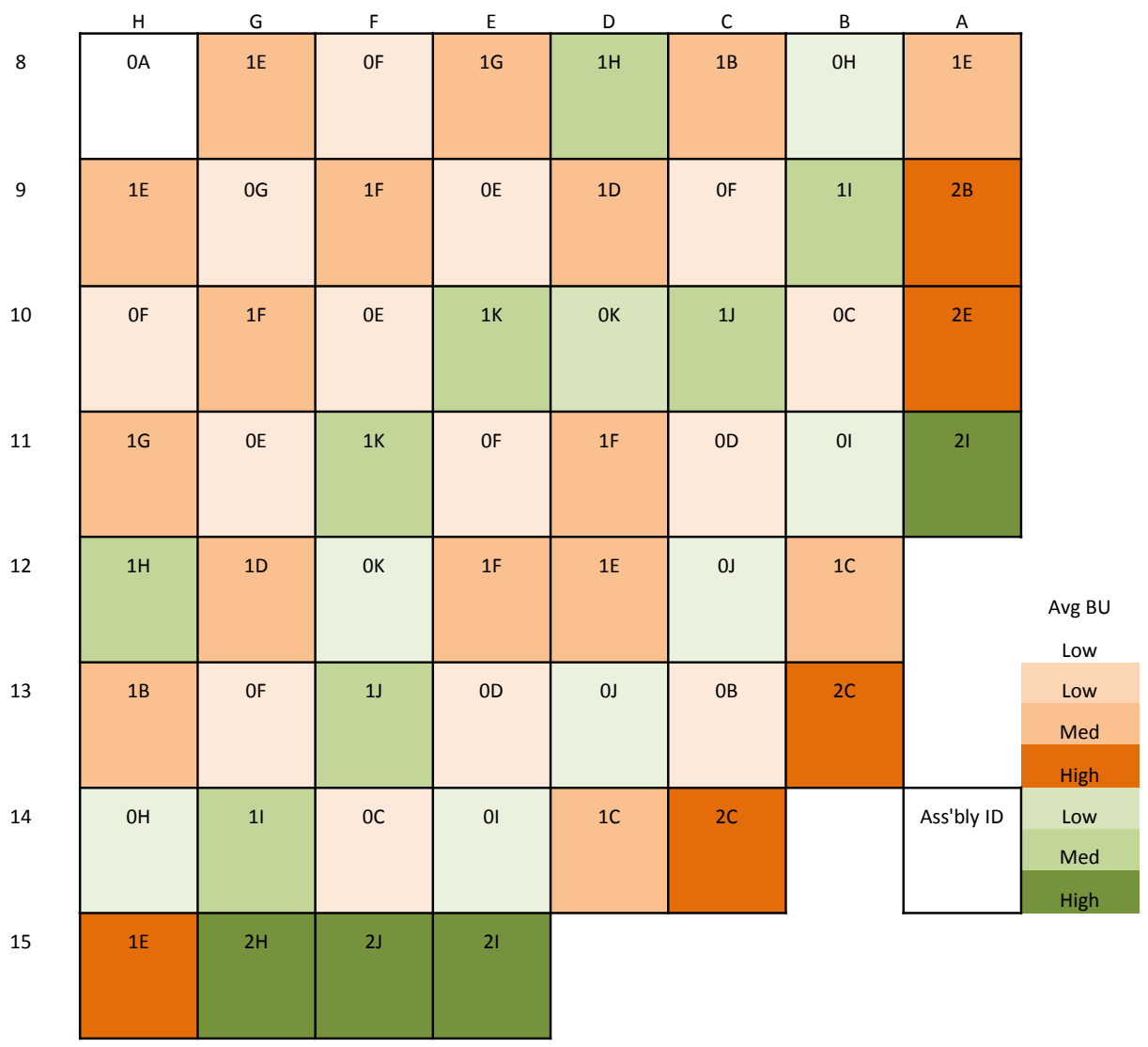

Figure 2. SQN equilibrium LEU core assembly identification map.

Table 2 summarizes the categories of assemblies by enrichment (color coded), burnup class, average burnup in burnup class, and fraction of each assembly category in the LEU core.

Table 2. Loading of the SQN equilibrium LEU core

\begin{tabular}{|c|c|c|c||}
\hline $\begin{array}{c}\text { Assembly } \\
\text { type }\end{array}$ & $\begin{array}{c}\text { Burnup } \\
\text { class }\end{array}$ & $\begin{array}{c}\text { Number of } \\
\text { assemblies in core }\end{array}$ & $\begin{array}{c}\text { Fraction in } \\
\text { core }\end{array}$ \\
\hline LEU & 1 & 1 & 0.0052 \\
\hline & 1 & 52 & 0.2694 \\
\hline & 2 & 28 & 0.1451 \\
\hline & 3 & 40 & 0.2073 \\
\hline & 1 & 28 & 0.1451 \\
\hline & 2 & 16 & 0.0829 \\
\hline & 3 & 28 & 0.1451 \\
\hline Total & & 193 & 1.0000 \\
\hline
\end{tabular}

\subsection{BFN MOX Core}

The BFN MOX core consists of both MOX and LEU fuel assemblies. Figure 3 presents a $1 / 4$ core map of the BFN equilibrium MOX core showing the assembly identifiers. Assembly identifiers contain either an "A," "B," "C," or "D." They have been highlighted in different colors to clarify their location and distribution in the core. Assemblies with " $A$ " or "B" in their identifiers are LEU assemblies. Assemblies with " $C$ " and " $D$ " in their identifiers are MOX assemblies. 


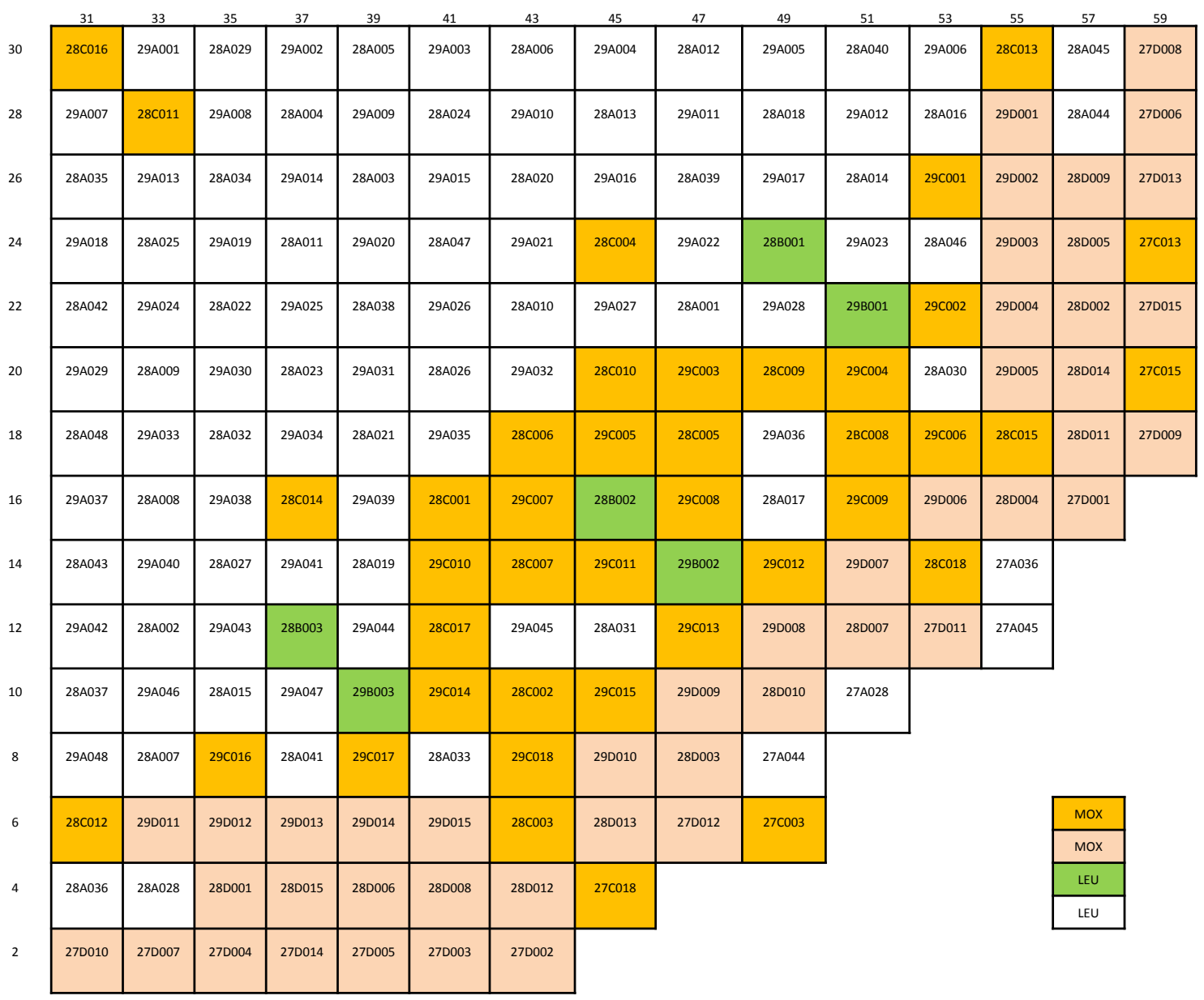

Figure 3. BFN MOX equilibrium core assay/enrichment and EOC burnup distribution.

Table 3 summarizes the categories of assemblies by assembly type, burnup class, average burnup in burnup class, and fraction of each assembly category in the MOX core. The color coding represents the assembly-average (Pu-Am) assay or the U-235 enrichment as applicable.

Table 3. Loading of the BFN MOX core

\begin{tabular}{|c|c|c|c||}
\hline $\begin{array}{c}\text { Assembly } \\
\text { type }\end{array}$ & $\begin{array}{c}\text { Burnup } \\
\text { class }\end{array}$ & $\begin{array}{c}\text { Number of } \\
\text { assemblies in core }\end{array}$ & $\begin{array}{c}\text { Fraction in } \\
\text { core }\end{array}$ \\
\hline \hline MOX & 1 & 72 & 0.0942 \\
\hline & 2 & 40 & 0.0524 \\
\hline & 3 & 48 & 0.0628 \\
\hline & 1 & 60 & 0.0785 \\
\hline & 2 & 52 & 0.0681 \\
\hline & 3 & 68 & 0.089 \\
\hline LEU & 1 & 12 & 0.0157 \\
\hline & 2 & 0 & 0 \\
\hline & 3 & 12 & 0.0157 \\
\hline & 1 & 192 & 0.2513 \\
\hline & 2 & 16 & 0.0209 \\
\hline & 3 & 192 & 0.2513 \\
\hline Total & & 764 & 1.0000 \\
\hline
\end{tabular}




\subsection{BFN MOX and LEU Fuel Assemblies}

The MOX and LEU assemblies in the BFN MOX core have a lower lattice (dominant) and an upper lattice (vanished). The MOX fuel assemblies have four MOX fuel rod assays. (LEU+Gd) rods have a variety of LEU enrichments, $\mathrm{Gd}_{2} \mathrm{O}_{3}$ concentrations, number and placements of Gd fuel rods. These variations were all modeled explicitly. The locations of the part-length rods are the same for all assemblies (MOX and LEU) in the BFN MOX core. Fuel assemblies typically have natural uranium fuel blankets at the top and bottom, as well as a plenum region where short rods are transitioning out. These regions are not modeled in this study.

\subsection{BFN LEU Core}

Figure 4 presents a $1 / 4$ core map of the BFN LEU equilibrium core showing the assembly identifiers which contain either an "A," "B," or "C". They have been highlighted in different colors to clarify their location and distribution in the core.

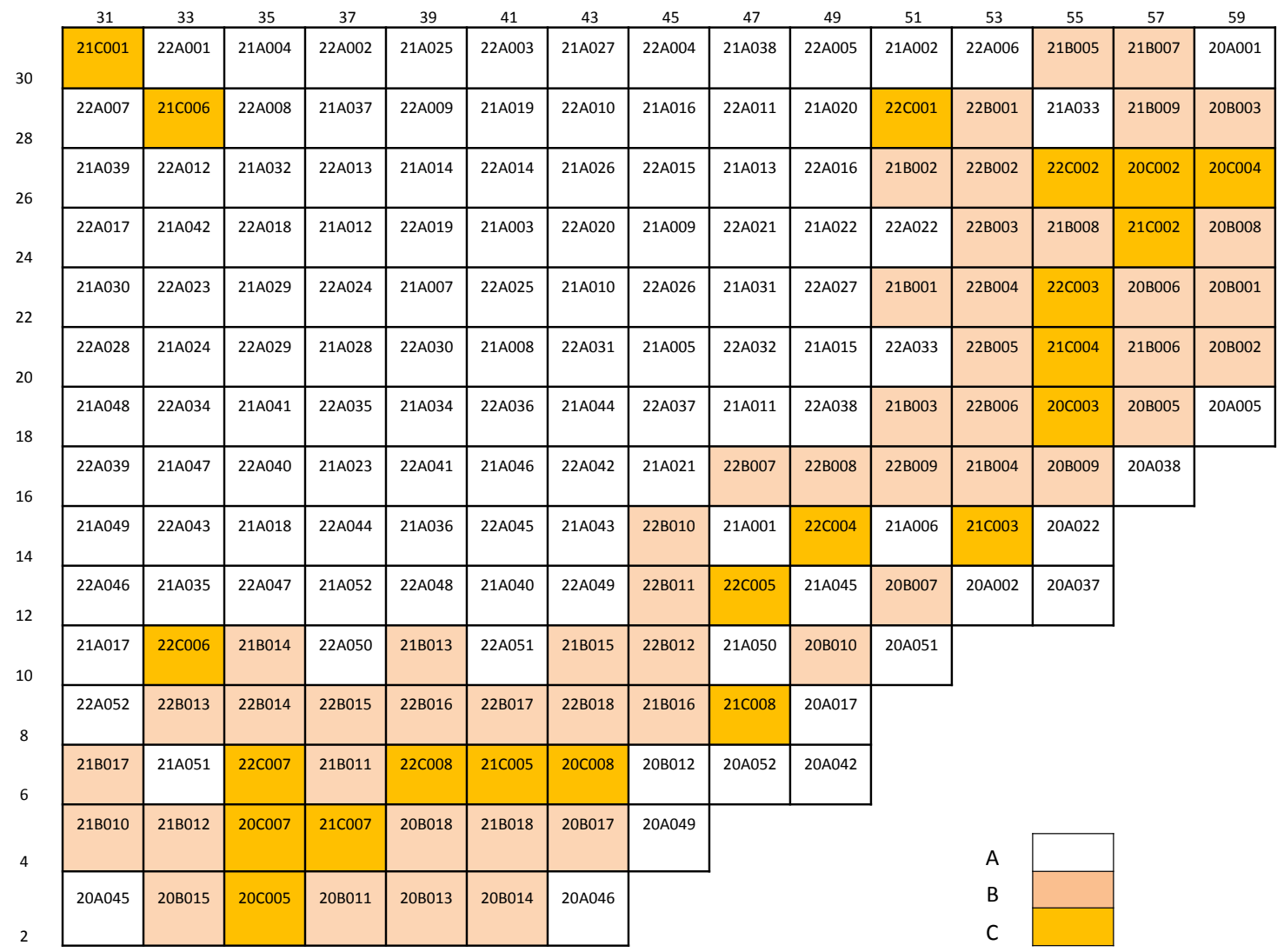

Figure 4. BFN equilibrium EOC burnup distribution in LEU core (GWd/MTHM).

Table 4 summarizes the categories of LEU assemblies by enrichments, average burnups in each burnup class, and the fraction in the core of each LEU assembly category. 
Table 4. Loading of BFN equilibrium LEU core

\begin{tabular}{||c|c|c|c||}
\hline $\begin{array}{c}\text { Assembly } \\
\text { type }\end{array}$ & $\begin{array}{c}\text { Burnup } \\
\text { classes }\end{array}$ & $\begin{array}{c}\text { Number of } \\
\text { assemblies in core }\end{array}$ & $\begin{array}{c}\text { Fraction in } \\
\text { core }\end{array}$ \\
\hline \hline LEU & 1 & 208 & 0.2723 \\
\hline & 2 & 20 & 0.0262 \\
\hline & 3 & 240 & 0.3141 \\
\hline & 1 & 72 & 0.0942 \\
\hline & 2 & 56 & 0.0733 \\
\hline & 3 & 80 & 0.1047 \\
\hline & 1 & 36 & 0.0471 \\
\hline & 2 & 24 & 0.0314 \\
\hline & 3 & 28 & 0.0367 \\
\hline Total & & 764 & 1.0000 \\
\hline
\end{tabular}

\section{BURNUP STEPS}

As previously mentioned, a maximum exposure of approximately $60 \mathrm{GWd} / \mathrm{MTHM}$ was chosen. The SQN fuel was depleted for 1700 days to reach a total burnup of $66.300 \mathrm{GWd} / \mathrm{MTHM}$. The BFN fuel was depleted for 2102 days to reach a total burnup of $60.958 \mathrm{GWd} / \mathrm{MTHM}$. The burnup steps used can be found in Table 5 .

Table 5. Burnup steps

\begin{tabular}{||c|c|c|c|c|c|c|c||}
\hline \multicolumn{3}{|c|}{ SQN } & \multicolumn{3}{c||}{ BFN } \\
\hline \hline $\begin{array}{c}\text { Time } \\
\text { (days) }\end{array}$ & $\begin{array}{c}\text { Burnup } \\
\text { (GWd/MTHM) }\end{array}$ & $\begin{array}{c}\text { Time } \\
\text { (days) }\end{array}$ & $\begin{array}{c}\text { Burnup } \\
\text { (GWd/MTHM) }\end{array}$ & $\begin{array}{c}\text { Time } \\
\text { (days) }\end{array}$ & $\begin{array}{c}\text { Burnup } \\
\text { (GWd/MTHM) }\end{array}$ & $\begin{array}{c}\text { Time } \\
\text { (days) }\end{array}$ & $\begin{array}{c}\text { Burnup } \\
\text { (GWd/MTHM) }\end{array}$ \\
\hline \hline 0 & 0.000 & 875 & 34.125 & 0 & 0.00 & 1052 & 30.508 \\
50 & 1.950 & 950 & 37.050 & 2 & 0.058 & 1202 & 34.858 \\
125 & 4.875 & 1025 & 39.975 & 92 & 2.668 & 1352 & 39.208 \\
200 & 7.800 & 1100 & 42.900 & 182 & 5.278 & 1502 & 43.558 \\
275 & 10.725 & 1175 & 45.825 & 272 & 7.888 & 1652 & 47.908 \\
350 & 13.650 & 1250 & 48.750 & 362 & 10.498 & 1802 & 52.258 \\
425 & 16.575 & 1325 & 51.675 & 452 & 13.108 & 1952 & 56.608 \\
500 & 19.500 & 1400 & 54.600 & 542 & 15.718 & 2102 & 60.958 \\
575 & 22.425 & 1475 & 57.525 & 632 & 18.328 & & \\
650 & 25.350 & 1550 & 60.450 & 722 & 20.938 & & \\
725 & 28.275 & 1625 & 63.375 & 812 & 23.548 & & \\
800 & 31.200 & 1700 & 66.300 & 902 & 26.158 & & \\
\hline
\end{tabular}

\section{CALCULATION PROCEDURE}

The computational analysis of the measurements was carried out using the two-dimensional (2D) depletion sequence T-DEPL of the TRITON module in the SCALE computer code system (version 6.1). The T-DEPL sequence in TRITON couples the 2D arbitrary polygonal mesh, discrete-ordinates transport code NEWT with the depletion and decay code ORIGEN-S in order to perform the burnup simulation. At each depletion step, the transport flux solution from NEWT is used to generate cross sections and assembly power distributions for the ORIGEN-S calculations; the isotopic composition data resulting 
from ORIGEN-S is employed in the subsequent transport calculation to obtain cross sections and power distributions for the next depletion step in an iterative manner throughout the irradiation history.

TRITON has the capability of simulating the depletion of multiple mixtures in a fuel assembly model. This is a very useful and powerful feature in a nuclide inventory analysis, as it allows a more appropriate representation of the local flux distribution and neutronic environment for a specific measured fuel rod in the assembly. The flux normalization in a TRITON calculation can be performed using, as a basis, the power in a specified mixture, the total power corresponding to multiple mixtures, or the assembly power. An average assembly power was used for these calculations.

Previous work $[9,10]$ has demonstrated the necessity to calculate individual fuel rod Dancoff factors when simulating highly heterogeneous BWR fuel assemblies. The built-in Dancoff factor routine in MIPLIB assumes a uniform distribution of uniform fuel rods, an assumption that is not appropriate in these cases. It was found that, for the ATRIUM 10 fuel assembly, the MCDANCOFF-calculated Dancoff factors were 25-30\% lower than SCALE/MIPLIB-calculated infinite lattice Dancoff factors for edge fuel rods and were up to $20 \%$ lower for some interior rods.

ORNL used the SCALE module MCDANCOFF and KENO-VI input files for the ATRIUM 10 assembly to calculate Dancoff factors for each pin in the dominant and vanished lattices for $0 \mathrm{wt} \%, 40 \mathrm{wt} \%$, and $80 \mathrm{wt} \%$ void concentrations.

The MCDANCOFF Dancoff factors are applied in the TRITON model during the CENTRM cross section processing step by using the dan2pitch(N) fuel option, where $\mathrm{N}$ is the fuel material identifier number whose Dancoff factor is being modified.

The W17 HTP assembly lattice is sufficiently homogeneous in a geometrical sense, and the Dancoff factors calculated by MIPLIB are acceptable.

All TRITON calculations employed the SCALE 238-group cross-section library based on ENDF/B-VII data collapsed to 49 groups ("weight" option in TRITON parm statement), for the pin-cell cross section treatment. Default values were used for the convergence parameters in the NEWT transport calculation.

\section{RESULTS}

\subsection{Dancoff Factors}

Dancoff factors were determined based on data obtained from SCALE/MCDANCOFF simulations as previously discussed in this report. The Dancoff factors corresponding to $25 \mathrm{vol} \%$ and $70 \mathrm{vol} \%$ void values were calculated from the fitting of the data calculated with MCDANCOFF for three pre-calculated sets at void values $(0,40$, and $80 \mathrm{vol} \%)$. To reduce the number of self-shielding calculations in the depletion simulation, a single value was used for the Dancoff factors for edge rods (the corner rods excluded), which was calculated as an average over the values for all edge rods; this assumption was used since there is little difference among the individual calculated Dancoff factors for the edge rods. The use of an average Dancoff factor for the corner rods was determined to be inappropriate, as the Dancoff factors for the corner rods are significantly different one from the other; therefore, individual Dancoff factors were used for the corner rods. 


\subsection{Multiplication Factor $\left(\mathbf{k}_{*}\right)$}

SQN

Figure 5 presents the infinite medium multiplication factor $\left(\mathrm{k}_{*}\right)$ trajectories for LEU fuel assemblies with different enrichments that are present in the SQN LEU equilibrium core. Figure 6 presents the $\mathrm{k}_{*}$ trajectories for the LEU and MOX fuel assemblies present in the SQN equilibrium MOX core. The rise in reactivity (up to approximately $8 \mathrm{GWd} / \mathrm{MTHM}$ in this case) is characteristic of assemblies that include gadolinia rods, resulting from the burnout of the gadolinium. Once the gadolinium has been consumed, the reactivity decreases steadily as fissile material is consumed at a rate greater than it is created.

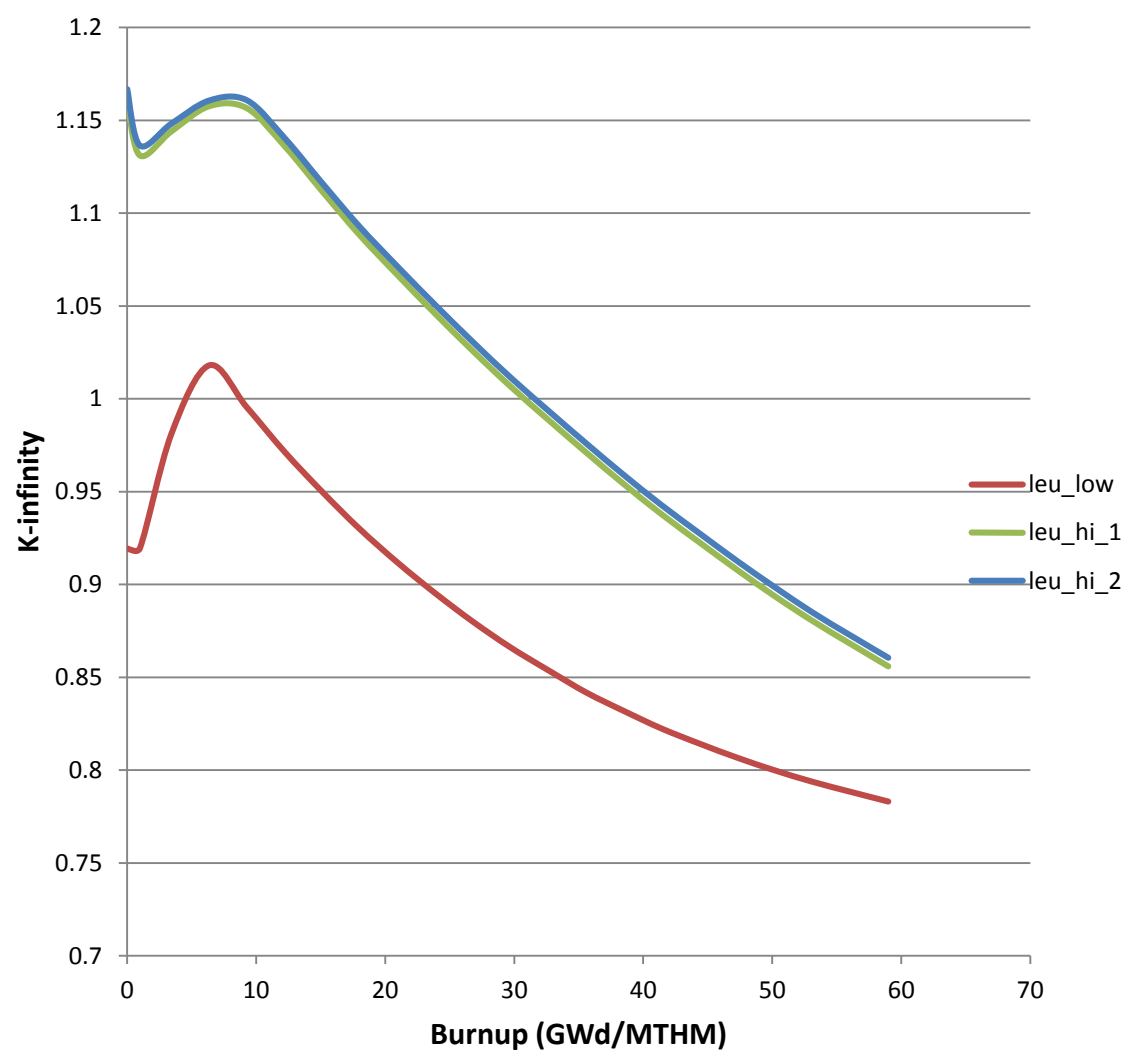

Figure 5. $k$, trajectories for SQN LEU core assemblies. 


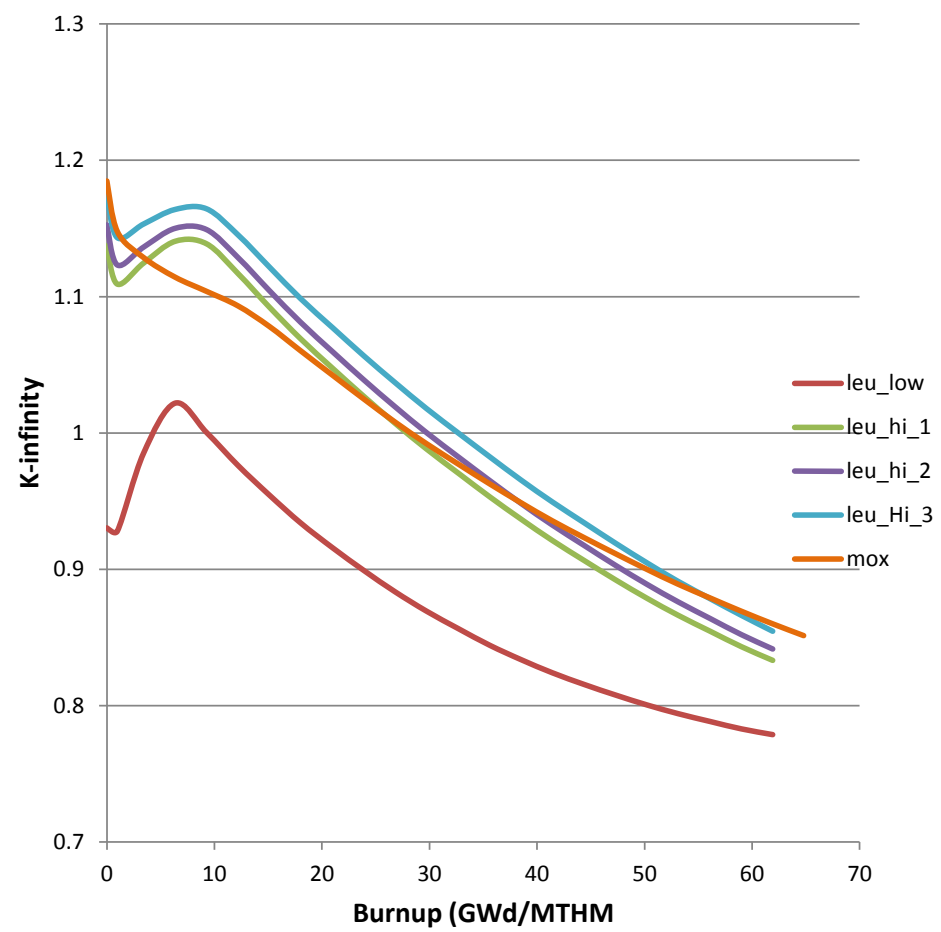

Figure 6. $k_{w}$ trajectories for SQN MOX core assemblies.

\section{BFN}

Figure 7 and Figure 8 present the $\mathrm{k}_{\mathrm{x}}$ trajectories of the dominant and vanished zones of the three LEU assemblies in the BFN equilibrium LEU core. The reactivity peaks occur at higher burnup than for the SQN assemblies due to higher loading of gadolinia.

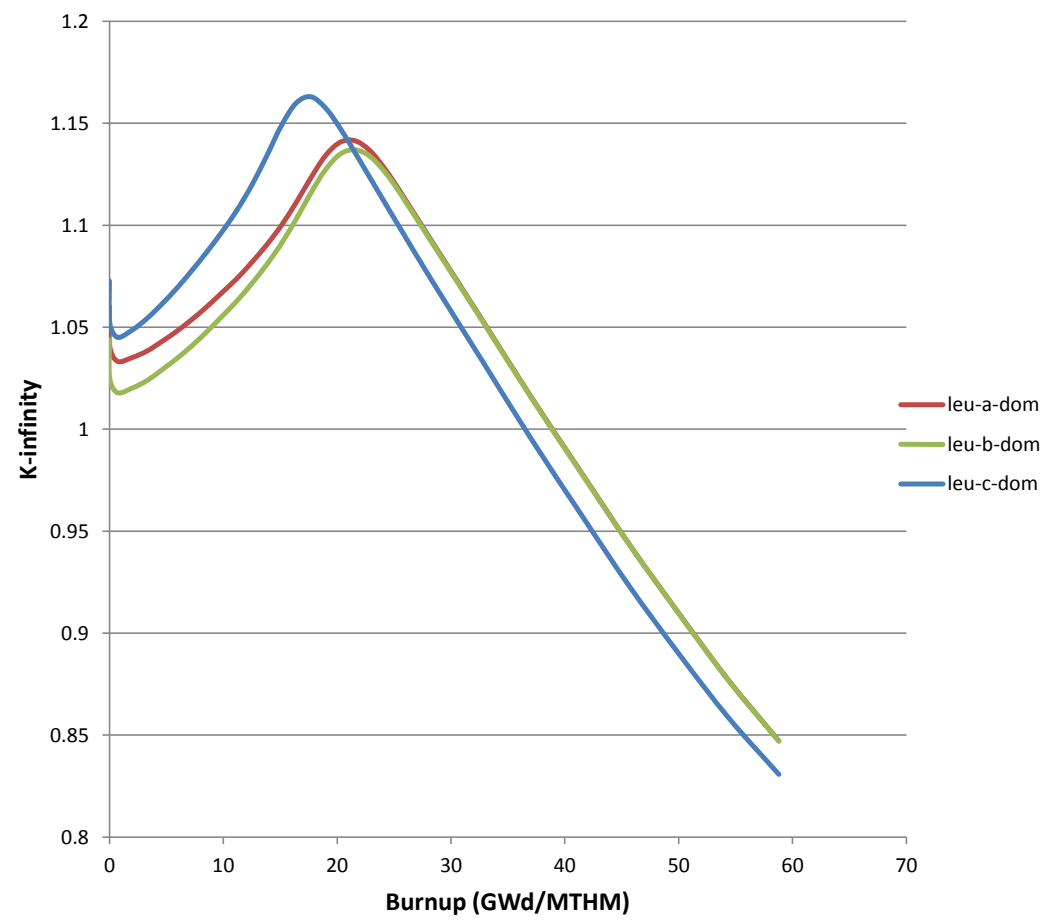

Figure 7. $k$, trajectories for BFN LEU core assemblies - dominant zone. 


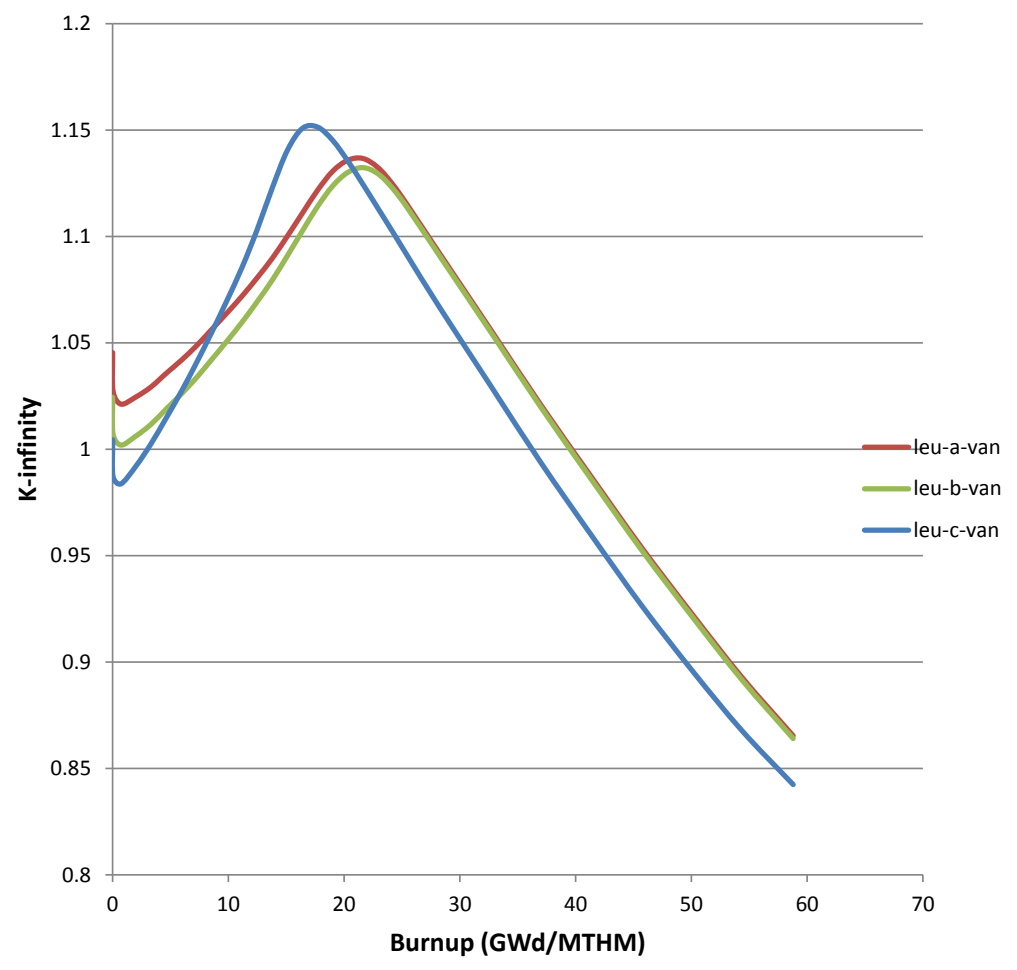

Figure 8. $k_{\text {w }}$ trajectories for BFN LEU core assemblies - vanished zone.

Figure 9 and Figure 10 present the $\mathrm{k}_{*}$ trajectories of the dominant and vanished zones of the two LEU assemblies and the two MOX assemblies in the BFN equilibrium MOX core.

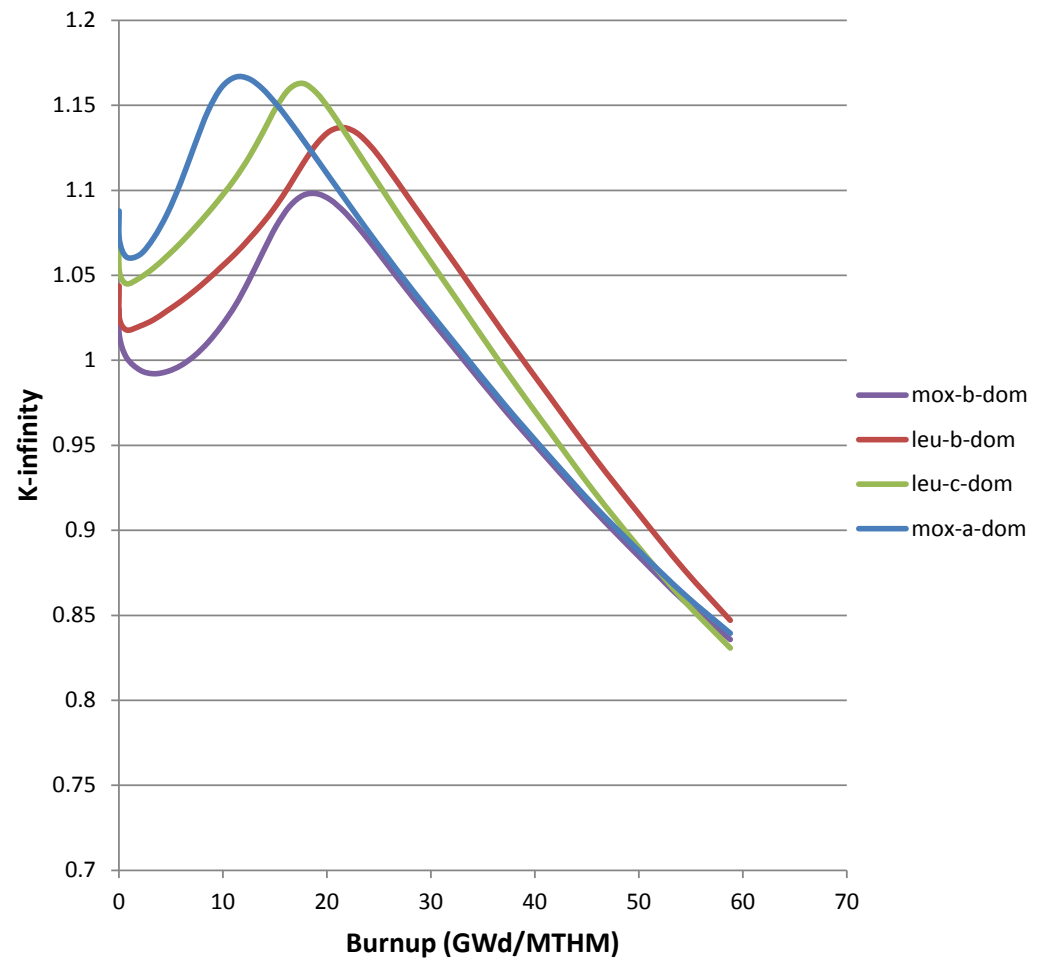

Figure 9. $k$. trajectories for BFN MOX core assemblies - dominant zone. 


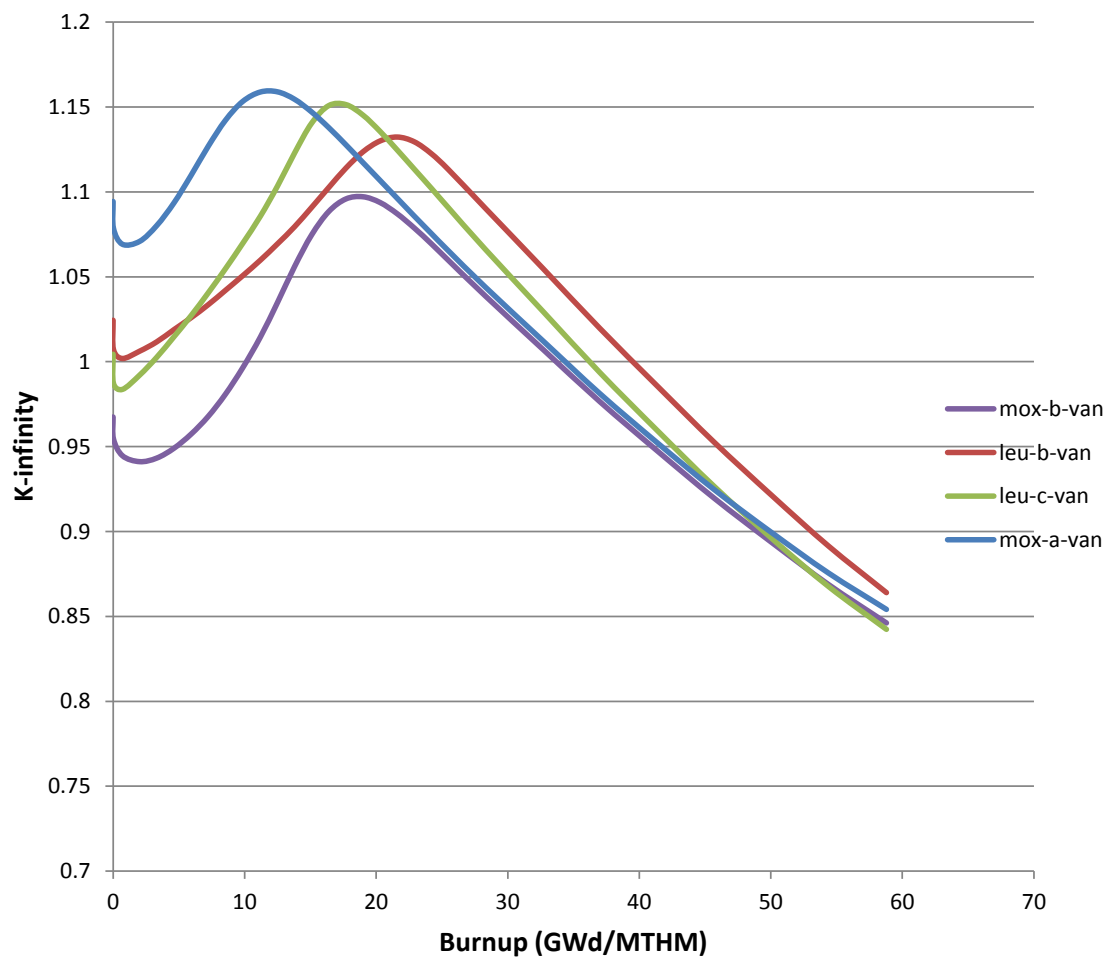

Figure 10. $k$, trajectories for BFN MOX core assemblies - vanished zone.

\subsection{Isotopic Masses and Isotopic Ratios}

\section{SQN}

Table 6 presents a summary of the total mass of each of the considered nuclides in the SQN MOX and LEU cores as well as the ratios of the nuclide contents in the MOX core and the LEU core. The total isotopic masses in a core were determined by multiplying the core-weighted-average isotopic mass per MTHM by the number of tonnes in each core (87.302 MTHM in the MOX core, 87.921 MTHM in the LEU core).

Table 6. Summary of nuclide concentrations for the SQN equilibrium MOX and LEU cores

\begin{tabular}{|c|c|c|c|c|c|c|c|}
\hline \multirow{4}{*}{ Isotope } & \multicolumn{4}{|c|}{ MOX core } & \multicolumn{2}{|c|}{ LEU core } & \multirow{4}{*}{ Ratios } \\
\hline & \multicolumn{3}{|c|}{ Core-weighted-average components } & \multirow[b]{2}{*}{ Total mass ${ }^{a}$} & \multirow{2}{*}{$\begin{array}{c}\text { Core-weighted } \\
\text { average of LEU } \\
\text { assemblies }\end{array}$} & \multirow[b]{2}{*}{ Total mass $^{a}$} & \\
\hline & $\begin{array}{c}\text { MOX } \\
\text { assemblies } \\
\end{array}$ & $\begin{array}{c}\text { LEU } \\
\text { assemblies }\end{array}$ & $\begin{array}{c}\text { (MOX+LEU) } \\
\text { assemblies }\end{array}$ & & & & \\
\hline & (g/MTHМ) & (g/MTHМ) & (g/MTHМ) & (g) & (g/MTHM) & (g) & \\
\hline $\operatorname{am} 241$ & $6.1300 \mathrm{E}+01$ & $2.8995 \mathrm{E}+01$ & $9.0294 \mathrm{E}+01$ & $7.8828 \mathrm{E}+03$ & $4.4701 \mathrm{E}+01$ & $3.9302 \mathrm{E}+03$ & 2.0199 \\
\hline $\operatorname{am} 242$ & $1.1532 \mathrm{E}-01$ & 7.5635E-02 & $1.9096 \mathrm{E}-01$ & $1.6671 \mathrm{E}+01$ & $1.1608 \mathrm{E}-01$ & $1.0206 \mathrm{E}+01$ & 1.6450 \\
\hline $\mathrm{am} 242 \mathrm{~m}$ & $1.0313 \mathrm{E}+00$ & $4.3809 \mathrm{E}-01$ & $1.4694 \mathrm{E}+00$ & $1.2828 \mathrm{E}+02$ & $6.7310 \mathrm{E}-01$ & $5.9180 \mathrm{E}+01$ & 2.1830 \\
\hline am243 & $9.3154 \mathrm{E}+01$ & $8.3508 \mathrm{E}+01$ & $1.7666 \mathrm{E}+02$ & $1.5423 \mathrm{E}+04$ & $1.2962 \mathrm{E}+02$ & $1.1396 \mathrm{E}+04$ & 1.3630 \\
\hline $\operatorname{am} 244$ & 5.2949E-03 & $5.3120 \mathrm{E}-03$ & $1.0607 \mathrm{E}-02$ & 9.2600E-01 & $8.2218 \mathrm{E}-03$ & $7.2287 \mathrm{E}-01$ & 1.2901 \\
\hline am245 & $1.3786 \mathrm{E}-06$ & $1.8349 \mathrm{E}-06$ & $3.2135 \mathrm{E}-06$ & 2.8054E-04 & $2.8557 \mathrm{E}-06$ & $2.5108 \mathrm{E}-04$ & 1.1253 \\
\hline ba $137 \mathrm{~m}$ & 7.6202E-05 & $1.4121 \mathrm{E}-04$ & $2.1741 \mathrm{E}-04$ & 1.8980E-02 & $2.2221 \mathrm{E}-04$ & $1.9537 \mathrm{E}-02$ & 0.9784 \\
\hline ba139 & $4.1130 \mathrm{E}-02$ & $7.2602 \mathrm{E}-02$ & $1.1373 \mathrm{E}-01$ & $9.9289 \mathrm{E}+00$ & $1.1616 \mathrm{E}-01$ & $1.0213 \mathrm{E}+01$ & 0.9791 \\
\hline ba140 & $8.7379 \mathrm{E}+00$ & $1.5551 \mathrm{E}+01$ & $2.4289 \mathrm{E}+01$ & $2.1205 \mathrm{E}+03$ & $2.4893 \mathrm{E}+01$ & $2.1886 \mathrm{E}+03$ & 0.9757 \\
\hline
\end{tabular}


Table 6 (continued)

\begin{tabular}{|c|c|c|c|c|c|c|c|}
\hline \multirow{4}{*}{ Isotope } & \multicolumn{4}{|c|}{ MOX core } & \multicolumn{2}{|c|}{ LEU core } & \multirow{4}{*}{ Ratios } \\
\hline & \multicolumn{3}{|c|}{ Core-weighted-average components } & \multirow[b]{2}{*}{ Total mass ${ }^{a}$} & \multirow{2}{*}{$\begin{array}{c}\text { Core-weighted } \\
\text { average of LEU } \\
\text { assemblies }\end{array}$} & \multirow[b]{2}{*}{ Total mass $^{a}$} & \\
\hline & $\begin{array}{c}\text { MOX } \\
\text { assemblies }\end{array}$ & $\begin{array}{c}\text { LEU } \\
\text { assemblies }\end{array}$ & $\begin{array}{c}\text { (MOX+LEU) } \\
\text { assemblies }\end{array}$ & & & & \\
\hline & (g/MTHM) & (g/MTHM) & (g/MTHM) & (g) & (g/MTHM) & (g) & \\
\hline ba141 & $8.1720 \mathrm{E}-03$ & $1.4570 \mathrm{E}-02$ & $2.2742 \mathrm{E}-02$ & $1.9854 \mathrm{E}+00$ & $2.3322 \mathrm{E}-02$ & $2.0505 \mathrm{E}+00$ & $\overline{0.9751}$ \\
\hline ba142 & $4.3390 \mathrm{E}-03$ & $7.9639 \mathrm{E}-03$ & & & & & 0.9637 \\
\hline br83 & $2.2175 \mathrm{E}-03$ & $4.8823 \mathrm{E}-03$ & & \begin{tabular}{|l|l|l}
$6.193 \mathrm{E}-01$ \\
\end{tabular} & $7.8858 \mathrm{E}-03$ & & 0.9003 \\
\hline br84 & $7.8295 \mathrm{E}-04$ & $1.8328 \mathrm{E}-03$ & $2.6157 \mathrm{E}-03$ & $2.2836 \mathrm{E}-01$ & $2.9658 \mathrm{E}-03$ & $2.6075 \mathrm{E}-01$ & 0.8820 \\
\hline br84m & $1.1391 \mathrm{E}-05$ & $1.5663 \mathrm{E}-05$ & $2.7054 \mathrm{E}-05$ & $2.3619 \mathrm{E}-03$ & $2.4757 \mathrm{E}-05$ & $2.1767 \mathrm{E}-03$ & 1.0928 \\
\hline br85 & $8.9117 \mathrm{E}-05$ & $2.2458 \mathrm{E}-04$ & $3.1370 \mathrm{E}-04$ & $2.7387 \mathrm{E}-02$ & $3.6453 \mathrm{E}-04$ & $3.2050 \mathrm{E}-02$ & 0.8606 \\
\hline br87 & $4.0578 \mathrm{E}-05$ & $1.0709 \mathrm{E}-04$ & $1.4767 \mathrm{E}-04$ & $1.2892 \mathrm{E}-02$ & $1.7402 \mathrm{E}-04$ & $1.5300 \mathrm{E}-02$ & 0.8486 \\
\hline cd112 & $9.2797 \mathrm{E}+00$ & $8.7546 \mathrm{E}+00$ & $1.8034 \mathrm{E}+01$ & $1.5744 \mathrm{E}+03$ & $1.3619 \mathrm{E}+01$ & $1.1974 \mathrm{E}+03$ & 1.3242 \\
\hline cd114 & $1.0056 \mathrm{E}+01$ & $1.0745 \mathrm{E}+01$ & $2.0802 \mathrm{E}+01$ & $1.8160 \mathrm{E}+03$ & $1.6747 \mathrm{E}+01$ & $1.4724 \mathrm{E}+03$ & 1.2421 \\
\hline ce140 & $4.5240 \mathrm{E}+02$ & $9.4872 \mathrm{E}+02$ & $1.4011 \mathrm{E}+03$ & $1.2232 \mathrm{E}+05$ & $1.4939 \mathrm{E}+03$ & & 0.9379 \\
\hline ce141 & $2.1070 \mathrm{E}+01$ & $3.7491 \mathrm{E}+01$ & & & $22 \mathrm{E}+01$ & & \\
\hline ce142 & $4.0173 \mathrm{E}+02$ & $8.5565 \mathrm{E}+02$ & & $1.0977 \mathrm{E}+05$ & $5 \mathrm{E}+03$ & & 0.9325 \\
\hline ce143 & $7.9138 \mathrm{E}-01$ & & & & & & \\
\hline ce144 & & & & & & & \\
\hline ce145 & & & & & & & \\
\hline $\mathrm{cm} 242$ & & & & & & & \\
\hline $\mathrm{cm} 2$ & & & & & & & \\
\hline $\mathrm{cm} 2$ & & & & & & & \\
\hline cm245 & & & & & & & \\
\hline cm246 & & & & & & & \\
\hline co58 & 5. & & & & & & \\
\hline \begin{tabular}{|l|}
$\operatorname{co} 60$ \\
\end{tabular} & 3. & & & & & & \\
\hline cs133 & +02 & 02 & & +05 & 03 & 05 & \\
\hline cs134 & $\mathrm{E}+01$ & 1. & 2 & $\mathrm{E}+04$ & $5 \mathrm{E}+02$ & $0 \mathrm{E}+04$ & 0.9632 \\
\hline cs135 & $67 \mathrm{E}+02$ & $\mathrm{E}+02$ & $E+02$ & $4.9248 \mathrm{E}+04$ & $5 \mathrm{E}+02$ & $473 E+04$ & 1.1409 \\
\hline cs136 & $91 \mathrm{E}-01$ & $6 \mathrm{E}-01$ & E-01 & $6.9890 \mathrm{E}+01$ & $7.1609 \mathrm{E}-01$ & $6.2959 \mathrm{E}+01$ & 1.1180 \\
\hline cs137 & $15 \mathrm{E}+02$ & $9.2049 \mathrm{E}+02$ & 03 & $359 \mathrm{E}+05$ & $85 \mathrm{E}+03$ & $1.2735 \mathrm{E}+05$ & 0.9773 \\
\hline cs138 & $1.7280 \mathrm{E}-02$ & $3.0422 \mathrm{E}-02$ & 02E-02 & $4.1645 \mathrm{E}+00$ & $4.8672 \mathrm{E}-02$ & $4.2793 \mathrm{E}+00$ & 0.9801 \\
\hline cs139 & 6E-03 & 176E-03 & $76 \mathrm{E}-02$ & $\mathrm{E}+00$ & $1.2673 \mathrm{E}-02$ & $1.1142 \mathrm{E}+00$ & 0.9766 \\
\hline cs140 & 4.0784E-04 & $7.6409 \mathrm{E}-04$ & 719E-03 & $1.0231 \mathrm{E}-01$ & $1.2253 \mathrm{E}-03$ & $1.0773 \mathrm{E}-01$ & 0.9565 \\
\hline cs141 & 302E-04 & $2.3821 \mathrm{E}-04$ & $123 \mathrm{E}-04$ & 3.2409E-02 & $3.8123 \mathrm{E}-04$ & $3.3518 \mathrm{E}-02$ & 0.9738 \\
\hline eu151 & $1.5693 \mathrm{E}-02$ & $1.0511 \mathrm{E}-02$ & $2.6203 \mathrm{E}-02$ & $2.2876 \mathrm{E}+00$ & $1.7013 \mathrm{E}-02$ & $1.4958 \mathrm{E}+00$ & 1.5402 \\
\hline eu153 & $5.8823 \mathrm{E}+01$ & $8.5814 \mathrm{E}+01$ & $1.4464 \mathrm{E}+02$ & $1.2627 \mathrm{E}+04$ & $1.3371 \mathrm{E}+02$ & $1.1756 \mathrm{E}+04$ & 1.0817 \\
\hline eu154 & $1.6079 \mathrm{E}+01$ & $2.0091 \mathrm{E}+01$ & $3.6170 \mathrm{E}+01$ & $3.1577 \mathrm{E}+03$ & $3.1108 \mathrm{E}+01$ & $2.7350 \mathrm{E}+03$ & 1.1627 \\
\hline eu155 & $4.4360 \mathrm{E}+00$ & $6.1459 \mathrm{E}+00$ & $1.0582 \mathrm{E}+01$ & $9.2382 \mathrm{E}+02$ & $9.5470 \mathrm{E}+00$ & $8.3938 \mathrm{E}+02$ & 1.1084 \\
\hline gd154 & $1.1160 \mathrm{E}+01$ & $1.7226 \mathrm{E}+01$ & $2.8386 \mathrm{E}+01$ & $2.4782 \mathrm{E}+03$ & $2.7593 \mathrm{E}+01$ & $2.4260 \mathrm{E}+03$ & 1.0288 \\
\hline gd155 & $1.3644 \mathrm{E}-01$ & $1.4415 \mathrm{E}-01$ & $2.8058 \mathrm{E}-01$ & $2.4495 \mathrm{E}+01$ & $2.2953 \mathrm{E}-01$ & $2.0180 \mathrm{E}+01$ & 1.2224 \\
\hline gd156 & & & & & & & 1.0173 \\
\hline gd157 & & & & & & & \\
\hline gd158 & & & & & & & \\
\hline \begin{tabular}{|l|}
$\mathrm{i} 129$ \\
\end{tabular} & & & & & & & 1.1350 \\
\hline i130 & 3. & & & & & & 1.0258 \\
\hline i131 & & $5.3226 \mathrm{E}+00$ & $3 \mathrm{E}+00$ & & $8.4738 \mathrm{E}+00$ & & 1.0236 \\
\hline i132 & $5.7863 \mathrm{E}-02$ & $9.3432 \mathrm{E}-02$ & $1.5130 \mathrm{E}-01$ & $1.3208 \mathrm{E}+01$ & $1.4885 \mathrm{E}-01$ & $1.3087 \mathrm{E}+01$ & 1.0164 \\
\hline i133 & $7.0339 \mathrm{E}-01$ & $1.1884 \mathrm{E}+00$ & $1.8918 \mathrm{E}+00$ & $1.6515 \mathrm{E}+02$ & $1.8975 \mathrm{E}+00$ & $1.6683 \mathrm{E}+02$ & 0.9970 \\
\hline
\end{tabular}


Table 6 (continued)

\begin{tabular}{|c|c|c|c|c|c|c|c|}
\hline \multirow{4}{*}{ Isotope } & \multicolumn{4}{|c|}{ MOX core } & \multicolumn{2}{|c|}{ LEU core } & \multirow{4}{*}{ Ratios } \\
\hline & \multicolumn{3}{|c|}{ Core-weighted-average components } & \multirow[b]{2}{*}{ Total mass ${ }^{a}$} & \multirow{2}{*}{$\begin{array}{c}\text { Core-weighted } \\
\text { average of LEU } \\
\text { assemblies }\end{array}$} & \multirow[b]{2}{*}{ Total mass $^{a}$} & \\
\hline & $\begin{array}{c}\text { MOX } \\
\text { assemblies }\end{array}$ & $\begin{array}{c}\text { LEU } \\
\text { assemblies }\end{array}$ & $\begin{array}{c}\text { (MOX+LEU) } \\
\text { assemblies }\end{array}$ & & & & \\
\hline & (g/MTHM) & (g/MTHM) & (g/MTHM) & (g) & (g/MTHM) & (g) & \\
\hline i134 & $3.2549 \mathrm{E}-02$ & $5.6243 \mathrm{E}-02$ & $8.8791 \mathrm{E}-02$ & $7.7516 \mathrm{E}+00$ & $8.9898 \mathrm{E}-02$ & $7.9039 \mathrm{E}+00$ & 0.9877 \\
\hline i135 & $2.1708 \mathrm{E}-01$ & $3.6386 \mathrm{E}-01$ & & & & & 1.0005 \\
\hline i136m & $1.0945 \mathrm{E}-04$ & $1.6951 \mathrm{E}-04$ & & & $2.6944 \mathrm{E}-04$ & $2.3690 \mathrm{E}-02$ & 1.0353 \\
\hline kr83m & $1.6814 \mathrm{E}-03$ & $3.7055 \mathrm{E}-03$ & $5.3870 \mathrm{E}-03$ & 4.7029E-01 & $5.9831 \mathrm{E}-03$ & 5.2604E-01 & 0.9004 \\
\hline kr85 & $7.0252 \mathrm{E}+00$ & $2.3627 \mathrm{E}+01$ & $3.0652 \mathrm{E}+01$ & $2.6760 \mathrm{E}+03$ & $3.7385 \mathrm{E}+01$ & $3.2869 \mathrm{E}+03$ & 0.8199 \\
\hline kr85m & $7.9709 \mathrm{E}-03$ & $2.0104 \mathrm{E}-02$ & $2.8075 \mathrm{E}-02$ & $2.4510 \mathrm{E}+00$ & $3.2631 \mathrm{E}-02$ & $2.8689 \mathrm{E}+00$ & 0.8604 \\
\hline kr87 & $4.3830 \mathrm{E}-03$ & $1.1203 \mathrm{E}-02$ & $1.5586 \mathrm{E}-02$ & $1.3607 \mathrm{E}+00$ & $1.8190 \mathrm{E}-02$ & $1.5992 \mathrm{E}+00$ & 0.8569 \\
\hline kr88 & $1.2716 \mathrm{E}-02$ & $3.3429 \mathrm{E}-02$ & 4.6145E-02 & $4.0285 \mathrm{E}+00$ & $5.4331 \mathrm{E}-02$ & $4.7768 \mathrm{E}+00$ & 0.8493 \\
\hline kr89 & $2.8267 \mathrm{E}-04$ & $7.7710 \mathrm{E}-04$ & & $9.2520 \mathrm{E}-02$ & $1.2647 \mathrm{E}-03$ & & 0.8380 \\
\hline kr90 & $4.6862 \mathrm{E}-05$ & $1.3857 \mathrm{E}-04$ & $1.8543 \mathrm{E}-04$ & 1.6189E-02 & $2.2595 \mathrm{E}-04$ & $1.9865 \mathrm{E}-02$ & 0.8207 \\
\hline la139 & $4.5092 \mathrm{E}+02$ & $9.2486 \mathrm{E}+02$ & & & & & \\
\hline la140 & $1.2379 \mathrm{E}+00$ & $2.2228 \mathrm{E}+00$ & 00 & $3.0212 \mathrm{E}+02$ & $558 \mathrm{E}+00$ & & 0.9733 \\
\hline \begin{tabular}{|l|} 
la141 \\
\end{tabular} & & & & & & & \\
\hline la142 & & & & & & & \\
\hline \begin{tabular}{|l} 
la143 \\
\end{tabular} & & & & & & & \\
\hline $\mathrm{mo} 10 \mathrm{c}$ & & & & & & & \\
\hline mo92 & & & & & & & \\
\hline mo94 & & & & & & & \\
\hline mo95 & 2.0 & & & & & & \\
\hline mo96 & & & & & & & \\
\hline $\begin{array}{l}\text { mo97 } \\
\text { mos }\end{array}$ & 28 & $\mathrm{E}+02$ & & & & & \\
\hline mo98 & +02 & & & +04 & & & \\
\hline mo99 & 1.4 & 00 & & +02 & & & \\
\hline nb95 & 1.4 & 2.7 & & +03 & $\mathrm{E}+01$ & 03 & 0.9404 \\
\hline nb97 & E-02 & $4.1477 \mathrm{E}-02$ & E-02 & +00 & $9 \mathrm{E}-02$ & $5.8370 \mathrm{E}+00$ & 0.9750 \\
\hline nb97m & E-07 & $6.7889 \mathrm{E}-07$ & E-06 & $666 \mathrm{E}-04$ & $2 \mathrm{E}-06$ & E-05 & 1.1449 \\
\hline nd142 & $73 \mathrm{E}+00$ & $1.5360 \mathrm{E}+01$ & $927 \mathrm{E}+01$ & $70 \mathrm{E}+03$ & $6 \mathrm{E}+01$ & $2.1195 \mathrm{E}+03$ & 0.8681 \\
\hline nd143 & $2.9757 \mathrm{E}+02$ & $6.0438 \mathrm{E}+02$ & $195 \mathrm{E}+02$ & $7.8742 \mathrm{E}+04$ & $95 \mathrm{E}+02$ & $8.3960 \mathrm{E}+04$ & 0.9445 \\
\hline nd144 & $2.6138 \mathrm{E}+02$ & $7.2314 \mathrm{E}+02$ & $52 \mathrm{E}+02$ & $8.5950 \mathrm{E}+04$ & $349 \mathrm{E}+03$ & $9.9779 \mathrm{E}+04$ & 0.8675 \\
\hline nd145 & $2.3041 \mathrm{E}+02$ & $5.0147 \mathrm{E}+02$ & $7.3188 \mathrm{E}+02$ & $6.3895 \mathrm{E}+04$ & $128 \mathrm{E}+02$ & $6.9570 \mathrm{E}+04$ & 0.9249 \\
\hline nd146 & $2.4484 \mathrm{E}+02$ & $5.3753 \mathrm{E}+02$ & $237 \mathrm{E}+02$ & $6.8302 \mathrm{E}+04$ & $610 \mathrm{E}+02$ & $7.4390 \mathrm{E}+04$ & 0.9247 \\
\hline nd147 & $3.0168 \mathrm{E}+00$ & $5.2619 \mathrm{E}+00$ & $8.2787 \mathrm{E}+00$ & $7.2275 \mathrm{E}+02$ & $139 \mathrm{E}+00$ & $7.3976 \mathrm{E}+02$ & 0.9839 \\
\hline nd148 & $1.4519 \mathrm{E}+02$ & $2.8070 \mathrm{E}+02$ & $4.2589 \mathrm{E}+02$ & $3.7181 \mathrm{E}+04$ & $4.4171 \mathrm{E}+02$ & $3.8836 \mathrm{E}+04$ & 0.9642 \\
\hline nd150 & $8.4858 \mathrm{E}+01$ & $1.3318 \mathrm{E}+02$ & $2.1804 \mathrm{E}+02$ & $1.9035 \mathrm{E}+04$ & $2.0906 \mathrm{E}+02$ & $1.8381 \mathrm{E}+04$ & 1.0429 \\
\hline np237 & $6.1198 \mathrm{E}+01$ & $3.5749 \mathrm{E}+02$ & $4.1869 \mathrm{E}+02$ & $3.6553 \mathrm{E}+04$ & $5.5991 \mathrm{E}+02$ & $4.9228 \mathrm{E}+04$ & 0.7478 \\
\hline np238 & $1.6589 \mathrm{E}-01$ & $1.1191 \mathrm{E}+00$ & $1.2850 \mathrm{E}+00$ & $1.1218 \mathrm{E}+02$ & $1.7405 \mathrm{E}+00$ & $1.5303 \mathrm{E}+02$ & 0.7383 \\
\hline np239 & $3.3225 \mathrm{E}+01$ & $5.9415 \mathrm{E}+01$ & $9.2641 \mathrm{E}+01$ & $8.0877 \mathrm{E}+03$ & $9.3324 \mathrm{E}+01$ & $8.2051 \mathrm{E}+03$ & 0.9927 \\
\hline np240 & & & & & & & 0.9709 \\
\hline pd105 & & & & & & & 1.2880 \\
\hline pd107 & & & & & & & 1.4052 \\
\hline pd108 & & & & & & $1.4066 \mathrm{E}+04$ & 1.4363 \\
\hline pd110 & 4. & & & & & & 1.4190 \\
\hline pm147 & $7.0233 \mathrm{E}+01$ & $1.3400 \mathrm{E}+02$ & $2.0423 \mathrm{E}+02$ & & & & 0.9580 \\
\hline pr143 & $7.5340 \mathrm{E}+00$ & $1.4231 \mathrm{E}+01$ & $2.1765 \mathrm{E}+01$ & $1.9001 \mathrm{E}+03$ & $2.2842 \mathrm{E}+01$ & $2.0083 \mathrm{E}+03$ & 0.9528 \\
\hline pr144 & $5.3155 \mathrm{E}-03$ & $1.1329 \mathrm{E}-02$ & $1.6645 \mathrm{E}-02$ & $1.4531 \mathrm{E}+00$ & $1.8007 \mathrm{E}-02$ & $1.5832 \mathrm{E}+00$ & 0.9243 \\
\hline
\end{tabular}


Table 6 (continued)

\begin{tabular}{|c|c|c|c|c|c|c|c|}
\hline \multirow{4}{*}{ Isotope } & \multicolumn{4}{|c|}{ MOX core } & \multicolumn{2}{|c|}{ LEU core } & \multirow{4}{*}{ Ratios } \\
\hline & \multicolumn{3}{|c|}{ Core-weighted-average components } & \multirow[b]{2}{*}{ Total mass $^{a}$} & \multirow{2}{*}{$\begin{array}{c}\text { Core-weighted } \\
\text { average of LEU } \\
\text { assemblies }\end{array}$} & \multirow[b]{2}{*}{ Total mass ${ }^{a}$} & \\
\hline & $\begin{array}{c}\text { MOX } \\
\text { assemblies }\end{array}$ & $\begin{array}{c}\text { LEU } \\
\text { assemblies }\end{array}$ & $\begin{array}{c}\text { (MOX+LEU) } \\
\text { assemblies }\end{array}$ & & & & \\
\hline & (g/MTHM) & (g/MTHM) & (g/MTHM) & (g) & (g/MTHM) & (g) & \\
\hline pr145 & $1.0107 \mathrm{E}-01$ & $1.8769 \mathrm{E}-01$ & $2.8876 \mathrm{E}-01$ & $.5209 \mathrm{E}+01$ & $30007 \mathrm{~F} 01$ & $2.6461 \mathrm{E}+01$ & 0.9594 \\
\hline pu237 & $1.8641 \mathrm{E}-04$ & $3.8721 \mathrm{E}-04$ & & & 60514504 & & 0.9479 \\
\hline pu238 & $4.2724 \mathrm{E}+01$ & $1.3320 \mathrm{E}+02$ & & $1.5358 \mathrm{E}+04$ & $2.0818 \mathrm{E}+02$ & $1.8303 \mathrm{E}+04$ & 0.8450 \\
\hline pu239 & $6.1164 \mathrm{E}+03$ & $4.0054 \mathrm{E}+03$ & $1.0122 \mathrm{E}+04$ & $8.8365 \mathrm{E}+05$ & $6.3346 \mathrm{E}+03$ & $5.5695 \mathrm{E}+05$ & 1.5979 \\
\hline pu240 & $2.9011 \mathrm{E}+03$ & $1.5056 \mathrm{E}+03$ & $4.4067 \mathrm{E}+03$ & $3.8471 \mathrm{E}+05$ & $2.3371 \mathrm{E}+03$ & $2.0548 \mathrm{E}+05$ & 1.8855 \\
\hline pu241 & $1.5081 \mathrm{E}+03$ & $9.5997 \mathrm{E}+02$ & $2.4680 \mathrm{E}+03$ & $2.1546 \mathrm{E}+05$ & $1.4833 \mathrm{E}+03$ & $1.3041 \mathrm{E}+05$ & 1.6639 \\
\hline pu242 & $3.9209 \mathrm{E}+02$ & $3.4373 \mathrm{E}+02$ & $7.3582 \mathrm{E}+02$ & $6.4239 \mathrm{E}+04$ & $5.3001 \mathrm{E}+02$ & $4.6599 \mathrm{E}+04$ & 1.3883 \\
\hline pu243 & $1.0501 \mathrm{E}-01$ & $1.0713 \mathrm{E}-01$ & $2.1214 \mathrm{E}-01$ & $1.8520 \mathrm{E}+01$ & $1.6384 \mathrm{E}-01$ & $1.4405 \mathrm{E}+01$ & 1.2947 \\
\hline pu244 & $2.6306 \mathrm{E}-02$ & $2.5456 \mathrm{E}-02$ & $5.1762 \mathrm{E}-02$ & $4.5189 \mathrm{E}+00$ & $4.0133 \mathrm{E}-02$ & $3.5285 \mathrm{E}+00$ & 1.2898 \\
\hline pu245 & $1.8115 \mathrm{E}-06$ & $1.7834 \mathrm{E}-06$ & 3.5949E-06 & 3.1384E-04 & $2.8135 \mathrm{E}-06$ & $2.4736 \mathrm{E}-04$ & 1.2778 \\
\hline rb86 & & $2.3476 \mathrm{E}-02$ & & & & & \\
\hline $\mathrm{rb88}$ & & 03 & & & & & \\
\hline rb89 & & & & & & & \\
\hline rb90 & 2 & & & & & & \\
\hline rb90r & & & & & & & \\
\hline \begin{tabular}{|l|} 
rb91 \\
\end{tabular} & & & & & & & \\
\hline rh1c & & & & & & & \\
\hline $\begin{array}{l}\text { rh10 } \\
\end{array}$ & & & & & & & \\
\hline \begin{tabular}{|l|} 
rh10 \\
\end{tabular} & & & & & & & \\
\hline rh106 & & & & & & & \\
\hline \begin{tabular}{|l|} 
rh107 \\
\end{tabular} & & & & & & & \\
\hline ru101 & 3 & 2 & & & & & \\
\hline ru102 & $E+02$ & +02 & & +04 & 02 & & 1.0304 \\
\hline \begin{tabular}{|l|} 
ru103 \\
\end{tabular} & $\mathrm{E}+01$ & 3.3 & 01 & +03 & 01 & 03 & 1.0965 \\
\hline ru104 & $\mathrm{E}+02$ & $\mathrm{E}+02$ & $\mathrm{E}+02$ & $7 E+04$ & $2 \mathrm{E}+02$ & $5.6245 \mathrm{E}+04$ & 1.1878 \\
\hline ru105 & $4 \mathrm{E}-02$ & E-01 & $\mathrm{E}-01$ & $\mathrm{E}+01$ & $65 \mathrm{E}-01$ & $7 \mathrm{E}+01$ & 1.1624 \\
\hline ru106 & $\mathrm{E}+02$ & $620 \mathrm{E}+02$ & 02 & $2.0498 \mathrm{E}+04$ & $\mathrm{E}+02$ & $1.5836 \mathrm{E}+04$ & 1.3036 \\
\hline ru107 & $8.1240 \mathrm{E}-04$ & $9.0834 \mathrm{E}-04$ & $07 \mathrm{E}-03$ & $1.5022 \mathrm{E}-01$ & $31 \mathrm{E}-03$ & $1.2424 \mathrm{E}-01$ & 1.2177 \\
\hline sb125 & $\mathrm{E}+00$ & $6.5719 \mathrm{E}+00$ & $27 \mathrm{E}+01$ & $E+03$ & $65 \mathrm{E}+01$ & $9.0250 \mathrm{E}+02$ & 1.1619 \\
\hline sb127 & $1.8097 \mathrm{E}-01$ & $2.4298 \mathrm{E}-01$ & $396 \mathrm{E}-01$ & $3.7012 \mathrm{E}+01$ & $3.8330 \mathrm{E}-01$ & $3.3700 \mathrm{E}+01$ & 1.1061 \\
\hline sb129 & $2.5298 \mathrm{E}-02$ & $3.5388 \mathrm{E}-02$ & E-02 & $5.2979 \mathrm{E}+00$ & $5.5926 \mathrm{E}-02$ & $4.9171 \mathrm{E}+00$ & 1.0851 \\
\hline sb130 & $3.2638 \mathrm{E}-03$ & $5.1681 \mathrm{E}-03$ & $318 \mathrm{E}-03$ & 7.3611E-01 & $8.2242 \mathrm{E}-03$ & $7.2308 \mathrm{E}-01$ & 1.0252 \\
\hline sb130n & $5.1785 \mathrm{E}-04$ & $8.5985 \mathrm{E}-04$ & 1.3777E-03 & $1.2027 \mathrm{E}-01$ & $1.3717 \mathrm{E}-03$ & $1.2060 \mathrm{E}-01$ & 1.0043 \\
\hline sb133 & 3.6134E-04 & $7.0257 \mathrm{E}-04$ & $1.0639 \mathrm{E}-03$ & $9.2881 \mathrm{E}-02$ & $1.1273 \mathrm{E}-03$ & 9.9112E-02 & 0.9438 \\
\hline se84 & $7.2983 \mathrm{E}-05$ & $1.7326 \mathrm{E}-04$ & $2.4624 \mathrm{E}-04$ & 2.1497E-02 & $2.8050 \mathrm{E}-04$ & $2.4662 \mathrm{E}-02$ & 0.8779 \\
\hline sm147 & $2.4059 \mathrm{E}+01$ & $5.1192 \mathrm{E}+01$ & $7.5251 \mathrm{E}+01$ & $6.5695 \mathrm{E}+03$ & $8.0481 \mathrm{E}+01$ & $7.0759 \mathrm{E}+03$ & 0.9350 \\
\hline sm148 & $5.2764 \mathrm{E}+01$ & $1.0460 \mathrm{E}+02$ & $1.5736 \mathrm{E}+02$ & $1.3738 \mathrm{E}+04$ & $1.6368 \mathrm{E}+02$ & $1.4391 \mathrm{E}+04$ & 0.9614 \\
\hline sm149 & $1.7680 \mathrm{E}+00$ & & & & & & 1.2164 \\
\hline sm150 & & & & & & & 0.9813 \\
\hline $\begin{array}{l}\mathrm{sm} 151 \\
\end{array}$ & & & & & & & 1.2268 \\
\hline $\begin{array}{l}\mathrm{sm} 152 \\
\end{array}$ & & & & & & & 1.0765 \\
\hline $\begin{array}{l}\mathrm{sm} 154 \\
\end{array}$ & $2.3564 \mathrm{E}+01$ & & & & & & 1.1936 \\
\hline sn130 & $1.7041 \mathrm{E}-04$ & $3.0586 \mathrm{E}-04$ & & & & $4.3031 \mathrm{E}-02$ & 0.9731 \\
\hline sr89 & $7.4778 \mathrm{E}+00$ & $2.0159 \mathrm{E}+01$ & $2.7637 \mathrm{E}+01$ & $2.4127 \mathrm{E}+03$ & $3.2752 \mathrm{E}+01$ & $2.8796 \mathrm{E}+03$ & 0.8438 \\
\hline sr90 & $1.1536 \mathrm{E}+02$ & $4.1317 \mathrm{E}+02$ & $5.2853 \mathrm{E}+02$ & $4.6142 \mathrm{E}+04$ & $6.5380 \mathrm{E}+02$ & $5.7483 \mathrm{E}+04$ & 0.8084 \\
\hline
\end{tabular}


Table 6 (continued)

\begin{tabular}{|c|c|c|c|c|c|c|c|}
\hline \multirow{4}{*}{ Isotope } & \multicolumn{4}{|c|}{ MOX core } & \multicolumn{2}{|c|}{ LEU core } & \multirow{4}{*}{ Ratios } \\
\hline & \multicolumn{3}{|c|}{ Core-weighted-average components } & \multirow[b]{2}{*}{ Total mass ${ }^{a}$} & \multirow{2}{*}{\begin{tabular}{|c|} 
Core-weighted \\
average of LEU \\
assemblies
\end{tabular}} & \multirow[b]{2}{*}{ Total mass $^{a}$} & \\
\hline & $\begin{array}{c}\text { MOX } \\
\text { assemblies }\end{array}$ & $\begin{array}{c}\text { LEU } \\
\text { assemblies }\end{array}$ & $\begin{array}{c}\text { (MOX+LEU) } \\
\text { assemblies }\end{array}$ & & & & \\
\hline & (g/MTHM) & (g/MTHM) & (g/MTHM) & (g) & (g/MTHM) & (g) & \\
\hline sr91 & $8.5443 \mathrm{E}-02$ & $2.0755 \mathrm{E}-01$ & $2.9300 \mathrm{E}-01$ & $2.5579 \mathrm{E}+01$ & 3.3644E-01 & $2.9580 \mathrm{E}+01$ & 0.8709 \\
\hline sr92 & $2.7899 \mathrm{E}-02$ & $.3120 \mathrm{E}-02$ & 9.1019E-02 & $7.9461 \mathrm{E}+00$ & $1.0204 \mathrm{E}-01$ & $8.9714 \mathrm{E}+00$ & 0.8920 \\
\hline sr93 & $1.5747 \mathrm{E}-03$ & $3.3319 \mathrm{E}-03$ & $4.9066 \mathrm{E}-03$ & 4.2835E-01 & $5.3734 \mathrm{E}-03$ & 4.7244E-01 & 0.9131 \\
\hline sr94 & $2.7056 \mathrm{E}-04$ & $5.6272 \mathrm{E}-04$ & $8.3327 \mathrm{E}-04$ & $7.2746 \mathrm{E}-02$ & $9.0692 \mathrm{E}-04$ & 7.9737E-02 & 0.9188 \\
\hline tc101 & $5.3720 \mathrm{E}-03$ & $8.7257 \mathrm{E}-03$ & & $1.2308 \mathrm{E}+00$ & & $1.2224 \mathrm{E}+00$ & \\
\hline tc99 & $3.1008 \mathrm{E}+02$ & $5.9221 \mathrm{E}+02$ & $9.0230 \mathrm{E}+02$ & $7.8772 \mathrm{E}+04$ & & & \\
\hline tc99m & $1.2038 \mathrm{E}-01$ & & & & & & \\
\hline te $125 \mathrm{~m}$ & & & & & & & \\
\hline te127 & & & & & & & \\
\hline te12 & & & & & & & \\
\hline te 129 & & & & & & & \\
\hline te 12 & & & & & & & \\
\hline te131 & & & & & & & \\
\hline te $131 \mathrm{~m}$ & & & & & & & \\
\hline te132 & & & & & & & \\
\hline te 133 & 3. & & & & & & \\
\hline te133n & & & & & & & \\
\hline \begin{tabular}{|l|} 
te134 \\
\end{tabular} & & & & & & & \\
\hline u234 & $6.9425 \mathrm{E}+00$ & $\mathrm{z}+02$ & 02 & 0 & $\mathrm{E}+02$ & $23 \mathrm{E}+04$ & 0.6340 \\
\hline u235 & $\mathrm{E}+02$ & +03 & & $\mathrm{E}+05$ & $\mathrm{E}+04$ & & 0.6410 \\
\hline $\mathrm{u} 236$ & +02 & $\mathrm{E}+03$ & & $\mathrm{E}+05$ & $\mathrm{E}+03$ & 4.77 & 0.6603 \\
\hline u237 & $\mathrm{E}+00$ & $\mathrm{E}+00$ & $41 \mathrm{E}+00$ & $\mathrm{E}+02$ & $7 \mathrm{E}+01$ & $74 \mathrm{E}+03$ & 0.7304 \\
\hline $\mathrm{u} 238$ & $3.4718 \mathrm{E}+05$ & $\mathrm{E}+05$ & -05 & $\mathrm{E}+07$ & $9.2777 \mathrm{E}+05$ & $70 \mathrm{E}+07$ & 1.0013 \\
\hline u239 & $2.3031 \mathrm{E}-01$ & $4.1195 \mathrm{E}-01$ & $26 \mathrm{E}-01$ & $5.6071 \mathrm{E}+01$ & $6.4698 \mathrm{E}-01$ & $5.6883 \mathrm{E}+01$ & 0.9927 \\
\hline xe131n & $6.5406 \mathrm{E}-02$ & $1.0342 \mathrm{E}-01$ & $1.6883 \mathrm{E}-01$ & $1.4739 \mathrm{E}+01$ & $32 \mathrm{E}-01$ & $1.4447 \mathrm{E}+01$ & 1.0274 \\
\hline \begin{tabular}{|l|} 
xe133 \\
\end{tabular} & $4.0965 \mathrm{E}+00$ & $6.9020 \mathrm{E}+00$ & $1.0998 \mathrm{E}+01$ & $9.6018 \mathrm{E}+02$ & $1.1017 \mathrm{E}+01$ & $9.6858 \mathrm{E}+02$ & 0.9984 \\
\hline xe133m & $2.4525 \mathrm{E}-02$ & $3.8395 \mathrm{E}-02$ & $6.2920 \mathrm{E}-02$ & $5.4930 \mathrm{E}+00$ & $6.0992 \mathrm{E}-02$ & $5.3625 \mathrm{E}+00$ & 1.0316 \\
\hline xe135 & $1.2827 \mathrm{E}-01$ & $1.4647 \mathrm{E}-01$ & 474E-01 & $2.3985 \mathrm{E}+01$ & $03 \mathrm{E}-01$ & $2.0840 \mathrm{E}+01$ & 1.1591 \\
\hline xe135m & $1.5262 \mathrm{E}-03$ & $2.2916 \mathrm{E}-03$ & $178 \mathrm{E}-03$ & 3.3330E-01 & $3.6386 \mathrm{E}-03$ & 3.1991E-01 & 1.0492 \\
\hline xe137 & $1.9687 \mathrm{E}-03$ & & & 4.6478E-01 & & 4.7120E-01 & 0.9934 \\
\hline xe138 & & & & $1.5918 \mathrm{E}+00$ & & & \\
\hline xe139 & & & & & & & \\
\hline xe140 & & & & & & & \\
\hline y90 & & & & & & & \\
\hline y91 & & & & & & & \\
\hline y91m & 4.2 & & & $1.2601 \mathrm{E}+00$ & & $72 \mathrm{E}+00$ & 0.8709 \\
\hline y92 & $3.7543 \mathrm{E}-02$ & $8.4970 \mathrm{E}-02$ & $1.2251 \mathrm{E}-01$ & $1.0696 \mathrm{E}+01$ & $1.3739 \mathrm{E}-01$ & $79 \mathrm{E}+01$ & 0.8917 \\
\hline y93 & $1.3404 \mathrm{E}-01$ & $2.8131 \mathrm{E}-01$ & $4.1535 \mathrm{E}-01$ & $3.6261 \mathrm{E}+01$ & $4.5358 \mathrm{E}-01$ & $3.9879 \mathrm{E}+01$ & 0.9157 \\
\hline y94 & $4.5847 \mathrm{E}-03$ & $9.2416 \mathrm{E}-03$ & $1.3826 \mathrm{E}-02$ & $1.2071 \mathrm{E}+00$ & $1.4876 \mathrm{E}-02$ & $1.3079 \mathrm{E}+00$ & 0.9294 \\
\hline y95 & $2.7975 \mathrm{E}-03$ & $5.3961 \mathrm{E}-03$ & $8.1936 \mathrm{E}-03$ & \begin{tabular}{|l|}
$7.1531 \mathrm{E}-01$ \\
\end{tabular} & $8.6700 \mathrm{E}-03$ & $7.6228 \mathrm{E}-01$ & 0.9450 \\
\hline y96 & $1.3655 \mathrm{E}-05$ & $2.8094 \mathrm{E}-05$ & $4.1749 \mathrm{E}-05$ & 3.6448E-03 & $4.5217 \mathrm{E}-05$ & $3.9755 \mathrm{E}-03$ & 0.9233 \\
\hline zr95 & $2.5797 \mathrm{E}+01$ & $5.0229 \mathrm{E}+01$ & $7.6027 \mathrm{E}+01$ & $6.6373 \mathrm{E}+03$ & $8.0693 \mathrm{E}+01$ & $7.0946 \mathrm{E}+03$ & 0.9422 \\
\hline zr97 & $3.2069 \mathrm{E}-01$ & $5.7382 \mathrm{E}-01$ & $8.9451 \mathrm{E}-01$ & 7.8092E+01 & $9.1857 \mathrm{E}-01$ & $8.0762 \mathrm{E}+01$ & 0.9738 \\
\hline
\end{tabular}

${ }^{a}$ Core totals based on 87.302 MTHM in MOX core, 87.921 MTHM in LEU core. 
Figure 11 graphically presents the MOX core/LEU core nuclide ratio data given in Table 6 . The large difference that is apparent for Mo-92 is related to the quite different fission yields of this isotope for plutonium isotopes and U-235.
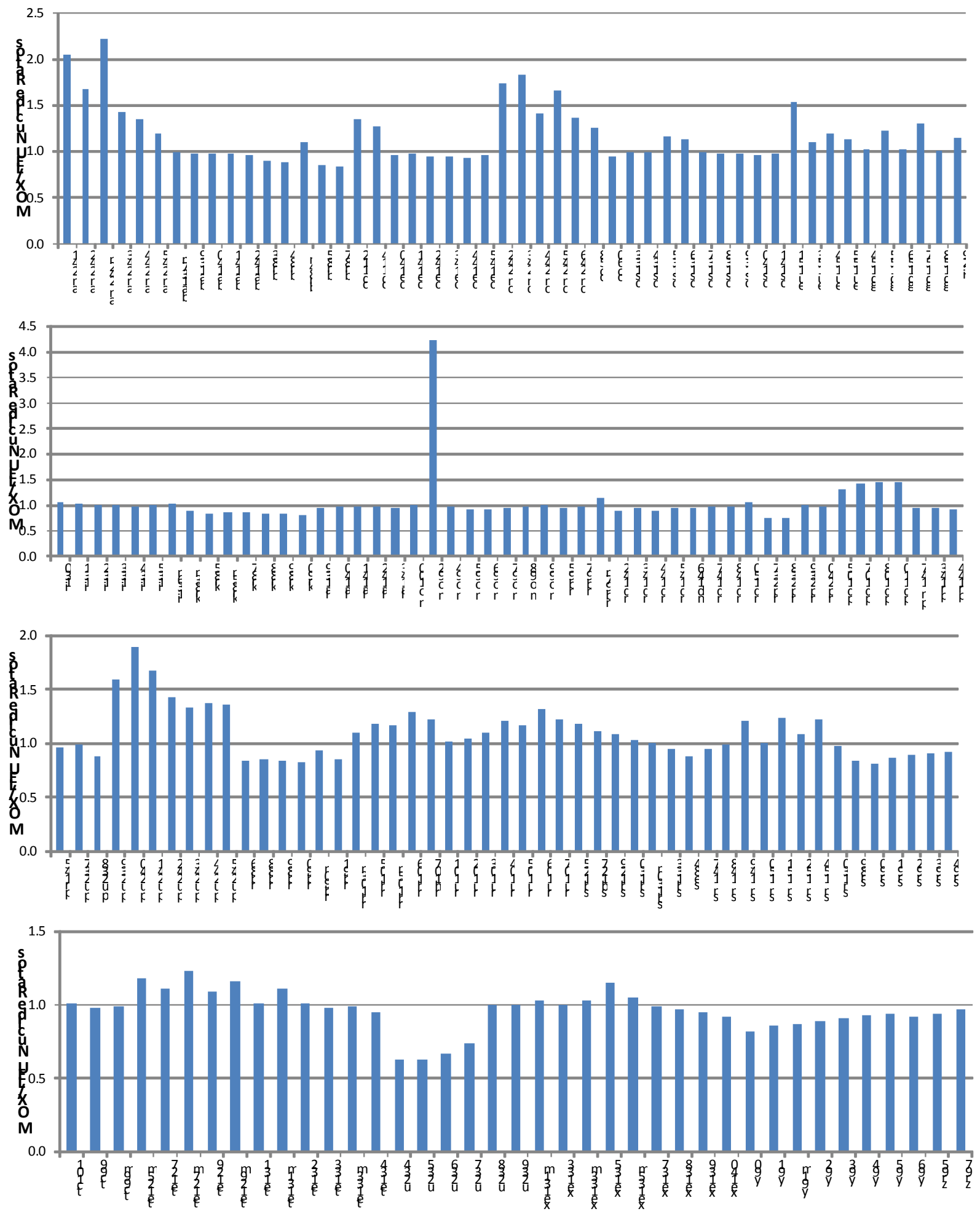

Figure 11. SQN MOX/LEU core-average nuclide ratios. 
Table 7 compares the SQN MOX core/LEU core nuclide ratios for the current study to the corresponding values reported in Reference [3]. The percentage difference is calculated as

$$
100 *\left(1-\mathrm{R}_{\mathrm{i}-\text { current }} / \mathrm{R}_{\mathrm{i} \text {-EIS-0283 }}\right) \text {. }
$$

Table 7. Comparison of SQN nuclide concentration ratios to Reference [3]

\begin{tabular}{|c|c|c|c|c|}
\hline \multirow{2}{*}{ Nuclide } & \multirow{2}{*}{$\begin{array}{c}\text { Current study } \\
\text { MOX/LEU ratios }\end{array}$} & \multicolumn{2}{|c|}{ EIS-0283 [3] } & \multirow{2}{*}{ Percentage difference } \\
\hline & & Isotopes & MOX/LEU ratios & \\
\hline am241 & 2.0199 & Americium-241 & 2.06 & 1.9 \\
\hline $\operatorname{am} 242$ & 1.6450 & & & \\
\hline am $242 \mathrm{~m}$ & 2.1830 & & & \\
\hline am243 & 1.3630 & & & \\
\hline am244 & 1.2901 & & & \\
\hline $\operatorname{am} 245$ & 1.1253 & & & \\
\hline ba137m & 0.9784 & & & \\
\hline ba139 & 0.9791 & Barium-139 & 0.97 & -0.9 \\
\hline ba140 & 0.9757 & Barium-140 & 0.98 & 0.4 \\
\hline ba141 & 0.9751 & & & \\
\hline ba142 & 0.9637 & & & \\
\hline br83 & 0.9003 & & & \\
\hline br84 & 0.8820 & & & \\
\hline br84m & 1.0928 & & & \\
\hline br85 & 0.8606 & & & \\
\hline br87 & 0.8486 & & & \\
\hline $\operatorname{cd} 112$ & 1.3242 & & & \\
\hline $\operatorname{cd} 114$ & 1.2421 & & & \\
\hline ce140 & 0.9379 & & & \\
\hline ce141 & 0.9757 & Cerium-141 & 0.98 & 0.4 \\
\hline ce142 & 0.9325 & & & \\
\hline ce143 & 0.9529 & Cerium-143 & 0.95 & -0.3 \\
\hline ce144 & 0.9245 & Cerium-144 & 0.91 & $\begin{array}{l}-1.6 \\
\end{array}$ \\
\hline ce145 & 0.9593 & & & \\
\hline $\mathrm{cm} 242$ & 1.6821 & Curium-242 & 1.43 & -17.6 \\
\hline $\mathrm{cm} 243$ & 1.7532 & & & \\
\hline $\mathrm{cm} 244$ & 1.3350 & Curium-244 & 0.94 & -42.0 \\
\hline $\mathrm{cm} 245$ & 1.5338 & & & \\
\hline $\mathrm{cm} 246$ & 1.2347 & & & \\
\hline $\operatorname{co5} 5$ & 1.3232 & Cobalt-58 & 0.86 & -53.9 \\
\hline co60 & 0.9244 & Cobalt-60 & 0.72 & -28.4 \\
\hline cs133 & 0.9765 & & & \\
\hline cs134 & 0.9632 & Cesium-134 & 0.85 & -13.3 \\
\hline cs135 & 1.1409 & & & \\
\hline cs136 & 1.1180 & Cesium-136 & 1.09 & -2.6 \\
\hline cs 137 & 0.9773 & Cesium-137 & 0.91 & -7.4 \\
\hline cs138 & 0.9801 & & & \\
\hline cs139 & 0.9766 & & & \\
\hline $\operatorname{cs} 140$ & 0.9565 & & & \\
\hline cs141 & 0.9738 & & & \\
\hline eu151 & 1.5402 & & & \\
\hline eu153 & 1.0817 & & & \\
\hline eu154 & 1.1627 & & & \\
\hline
\end{tabular}


Table 7 (continued)

\begin{tabular}{|c|c|c|c|c|}
\hline \multirow{2}{*}{ Nuclide } & \multirow{2}{*}{$\begin{array}{c}\text { Current study } \\
\text { MOX/LEU ratios }\end{array}$} & \multicolumn{2}{|c|}{ EIS-0283 [3] } & \multirow{2}{*}{ Percentage difference } \\
\hline & & Isotopes & MOX/LEU ratios & \\
\hline eu155 & 1.1084 & & & \\
\hline $\operatorname{gd} 154$ & 1.0288 & & & \\
\hline gd155 & 1.2224 & & & \\
\hline $\operatorname{gd} 156$ & 1.0173 & & & \\
\hline $\operatorname{gd} 157$ & 1.3042 & & & \\
\hline gd158 & 1.0139 & & & \\
\hline i129 & 1.1350 & & & \\
\hline $\mathrm{i} 130$ & 1.0258 & & & \\
\hline $\mathrm{i} 131$ & 1.0236 & Iodine-131 & 1.03 & 0.6 \\
\hline$\overline{\mathrm{i} 132}$ & 1.0164 & Iodine-132 & 1.02 & 0.3 \\
\hline $\mathrm{i} 133$ & 0.9970 & Iodine-133 & 1 & 0.3 \\
\hline i134 & 0.9877 & Iodine-134 & 0.98 & -0.8 \\
\hline$\overline{\mathrm{i} 135}$ & 1.0005 & Iodine- 135 & 1 & $\begin{array}{l}-0.1 \\
\end{array}$ \\
\hline i136m & 1.0353 & & & \\
\hline $\mathrm{kr} 83 \mathrm{~m}$ & 0.9004 & Krypton-83m & 0.89 & -1.2 \\
\hline kr85 & 0.8199 & Krypton-85 & 0.78 & -5.1 \\
\hline $\mathrm{kr} 85 \mathrm{~m}$ & 0.8604 & Krypton- $85 \mathrm{~m}$ & 0.86 & 0.0 \\
\hline kr87 & 0.8569 & Krypton-87 & 0.85 & -0.8 \\
\hline $\mathrm{kr} 88$ & 0.8493 & Krypton- 88 & 0.84 & -1.1 \\
\hline kr89 & 0.8380 & & & \\
\hline kr90 & 0.8207 & & & \\
\hline la139 & 0.9444 & & & \\
\hline la140 & 0.9733 & Lanthanum-140 & 0.97 & -0.3 \\
\hline la141 & 0.9759 & Lanthanum-141 & 0.97 & -0.6 \\
\hline la142 & 0.9680 & Lanthanum-142 & 0.97 & 0.2 \\
\hline la143 & 0.9533 & & & \\
\hline mo100 & 0.9761 & & & \\
\hline mo92 & 3.9709 & & & \\
\hline mo94 & 0.9359 & & & \\
\hline mo95 & 0.9047 & & & \\
\hline mo96 & 0.8758 & & & \\
\hline mo97 & 0.9439 & & & \\
\hline mo98 & 0.9619 & & & \\
\hline mo99 & 0.9936 & Molybdenum-99 & 0.99 & -0.4 \\
\hline nb95 & 0.9404 & Niobium-95 & 0.94 & 0.0 \\
\hline nb97 & 0.9750 & & & \\
\hline nb97m & 1.1449 & & & \\
\hline nd142 & 0.8681 & & & \\
\hline nd143 & 0.9445 & & & \\
\hline nd144 & 0.8675 & & & \\
\hline nd145 & 0.9249 & & & \\
\hline nd146 & 0.9247 & & & \\
\hline nd148 & 0.9642 & & & \\
\hline nd150 & 1.0429 & & & \\
\hline np237 & 0.7478 & & & \\
\hline np238 & 0.7383 & & & \\
\hline np239 & 0.9927 & Neptunium-239 & 0.99 & -0.3 \\
\hline
\end{tabular}


Table 7 (continued)

\begin{tabular}{|c|c|c|c|c|}
\hline \multirow{2}{*}{ Nuclide } & \multirow{2}{*}{$\begin{array}{c}\text { Current study } \\
\text { MOX/LEU ratios }\end{array}$} & \multicolumn{2}{|c|}{ EIS-0283 [3] } & \multirow{2}{*}{ Percentage difference } \\
\hline & & Isotopes & MOX/LEU ratios & \\
\hline np240 & 0.9709 & & & \\
\hline $\mathrm{pd} 105$ & 1.2880 & & & \\
\hline pd107 & 1.4052 & & & \\
\hline pd108 & 1.4363 & & & \\
\hline pd110 & 1.4190 & & & \\
\hline pm147 & 0.9580 & & & \\
\hline pr143 & 0.9528 & Praseodymium-143 & 0.95 & -0.3 \\
\hline pr144 & 0.9243 & & & \\
\hline pr145 & 0.9594 & & & \\
\hline pu237 & 0.9479 & & & \\
\hline pu238 & 0.8450 & Plutonium-238 & 0.76 & -11.2 \\
\hline pu239 & 1.5979 & Plutonium-239 & 2.06 & 22.4 \\
\hline pu240 & 1.8855 & Plutonium-240 & 2.2 & 14.3 \\
\hline pu241 & 1.6639 & Plutonium-241 & 1.79 & 7.0 \\
\hline pu242 & 1.3883 & & & \\
\hline pu243 & 1.2947 & & & \\
\hline pu244 & 1.2898 & & & \\
\hline pu245 & 1.2778 & & & \\
\hline $\mathrm{rb} 86$ & 0.8240 & Rubidium-86 & 0.77 & -7.0 \\
\hline $\mathrm{rb} 88$ & 0.8529 & & & \\
\hline $\mathrm{rb89}$ & 0.8489 & & & \\
\hline rb90 & 0.8276 & & & \\
\hline $\mathrm{rb} 90 \mathrm{~m}$ & 0.9442 & & & \\
\hline $\mathrm{rb91}$ & 0.8603 & & & \\
\hline rh103m & 1.0966 & & & \\
\hline rh105 & 1.1726 & Rhodium-105 & 1.19 & 1.5 \\
\hline rh105m & 1.1626 & & & \\
\hline rh106 & 1.2713 & & & \\
\hline rh107 & 1.2175 & & & \\
\hline ru101 & 0.9946 & & & \\
\hline ru102 & 1.0304 & & & \\
\hline ru103 & 1.0965 & Ruthenium-103 & 1.11 & 1.2 \\
\hline ru104 & 1.1878 & & & \\
\hline ru105 & 1.1624 & Ruthenium-105 & 1.18 & 1.5 \\
\hline ru106 & 1.3036 & Ruthenium-106 & 1.28 & -1.8 \\
\hline ru107 & 1.2177 & & & \\
\hline sb125 & 1.1619 & & & \\
\hline sb127 & 1.1061 & Antimony-127 & 1.15 & 3.8 \\
\hline sb129 & 1.0851 & Antimony-129 & 1.07 & -1.4 \\
\hline sb130 & 1.0252 & & & \\
\hline sb133 & 0.9438 & & & \\
\hline se84 & 0.8779 & & & \\
\hline sm147 & 0.9350 & & & \\
\hline $\mathrm{sm} 148$ & 0.9614 & & & \\
\hline sm149 & 1.2164 & & & \\
\hline sm150 & 0.9813 & & & \\
\hline sm151 & 1.2268 & & & \\
\hline $\operatorname{sm} 152$ & 1.0765 & & & \\
\hline
\end{tabular}


Table 7 (continued)

\begin{tabular}{|c|c|c|c|c|}
\hline \multirow{2}{*}{ Nuclide } & \multirow{2}{*}{$\begin{array}{c}\text { Current study } \\
\text { MOX/LEU ratios }\end{array}$} & \multicolumn{2}{|c|}{ EIS-0283 [3] } & \multirow{2}{*}{ Percentage difference } \\
\hline & & Isotopes & MOX/LEU ratios & \\
\hline sm154 & 1.1936 & & & \\
\hline sn130 & 0.9731 & & & \\
\hline sr89 & 0.8438 & Strontium-89 & 0.83 & -1.7 \\
\hline sr90 & 0.8084 & Strontium-90 & 0.75 & -7.8 \\
\hline $\operatorname{sr} 91$ & 0.8709 & Strontium-91 & 0.86 & -1.3 \\
\hline sr92 & 0.8920 & Strontium-92 & 0.89 & -0.2 \\
\hline sr93 & 0.9131 & & & \\
\hline sr94 & 0.9188 & & & \\
\hline tc 101 & 1.0140 & & & \\
\hline tc99 & 0.9672 & & & \\
\hline tc99m & 0.9936 & Technetium-99m & 0.99 & -0.4 \\
\hline te $125 \mathrm{~m}$ & 1.1693 & & & \\
\hline te127 & 1.1097 & Tellurium-127 & 1.16 & 4.3 \\
\hline te $127 \mathrm{~m}$ & 1.2293 & Tellurium-127m & 1.2 & -2.4 \\
\hline te 129 & 1.0852 & Tellurium-129 & 1.08 & -0.5 \\
\hline te $129 \mathrm{~m}$ & 1.1520 & Tellurium-129m & 1.09 & -5.7 \\
\hline te131 & 1.0115 & & & \\
\hline te $131 \mathrm{~m}$ & 1.1108 & Tellurium-131m & 1.11 & -0.1 \\
\hline te132 & 1.0124 & Tellurium-132 & 1.01 & -0.2 \\
\hline te 133 & 0.9807 & & & \\
\hline te $133 \mathrm{~m}$ & 0.9884 & & & \\
\hline te134 & 0.9557 & & & \\
\hline u234 & 0.6340 & & & \\
\hline u235 & 0.6410 & & & \\
\hline $\mathrm{u} 236$ & 0.6603 & & & \\
\hline $\mathrm{u} 237$ & 0.7304 & & & \\
\hline $\mathrm{u} 238$ & 1.0013 & & & \\
\hline $\mathrm{u} 239$ & 0.9927 & & & \\
\hline xe $131 \mathrm{~m}$ & 1.0274 & Xenon- $131 \mathrm{~m}$ & 1.02 & -0.7 \\
\hline xe133 & 0.9984 & Xenon-133 & 1 & 0.2 \\
\hline xe133m & 1.0316 & Xenon-133m & 1.01 & -2.1 \\
\hline xe135 & 1.1591 & Xenon-135 & 1.28 & 9.4 \\
\hline xe135m & 1.0492 & Xenon-135m & 1.04 & -0.9 \\
\hline xe137 & 0.9934 & & & \\
\hline xe138 & 0.9735 & Xenon-138 & 0.96 & -1.4 \\
\hline xe139 & 0.9479 & & & \\
\hline xe140 & 0.9203 & & & \\
\hline y91 & 0.8650 & Yttrium-91 & 0.85 & -1.8 \\
\hline $\mathrm{y} 91 \mathrm{~m}$ & 0.8709 & & & \\
\hline $\mathrm{y} 92$ & 0.8917 & Yttrium-92 & 0.89 & -0.2 \\
\hline y93 & 0.9157 & Yttrium-93 & 0.91 & -0.6 \\
\hline y94 & 0.9294 & & & \\
\hline y95 & 0.9450 & & & \\
\hline y96 & 0.9233 & & & \\
\hline $\mathrm{zr} 95$ & 0.9422 & Zirconium-95 & 0.94 & -0.2 \\
\hline zr97 & 0.9738 & Zirconium-97 & 0.98 & 0.6 \\
\hline
\end{tabular}


Figure 12 presents the percentage differences of the MOX/LEU ratios of the current study to those of Reference [3] given in Table 7.
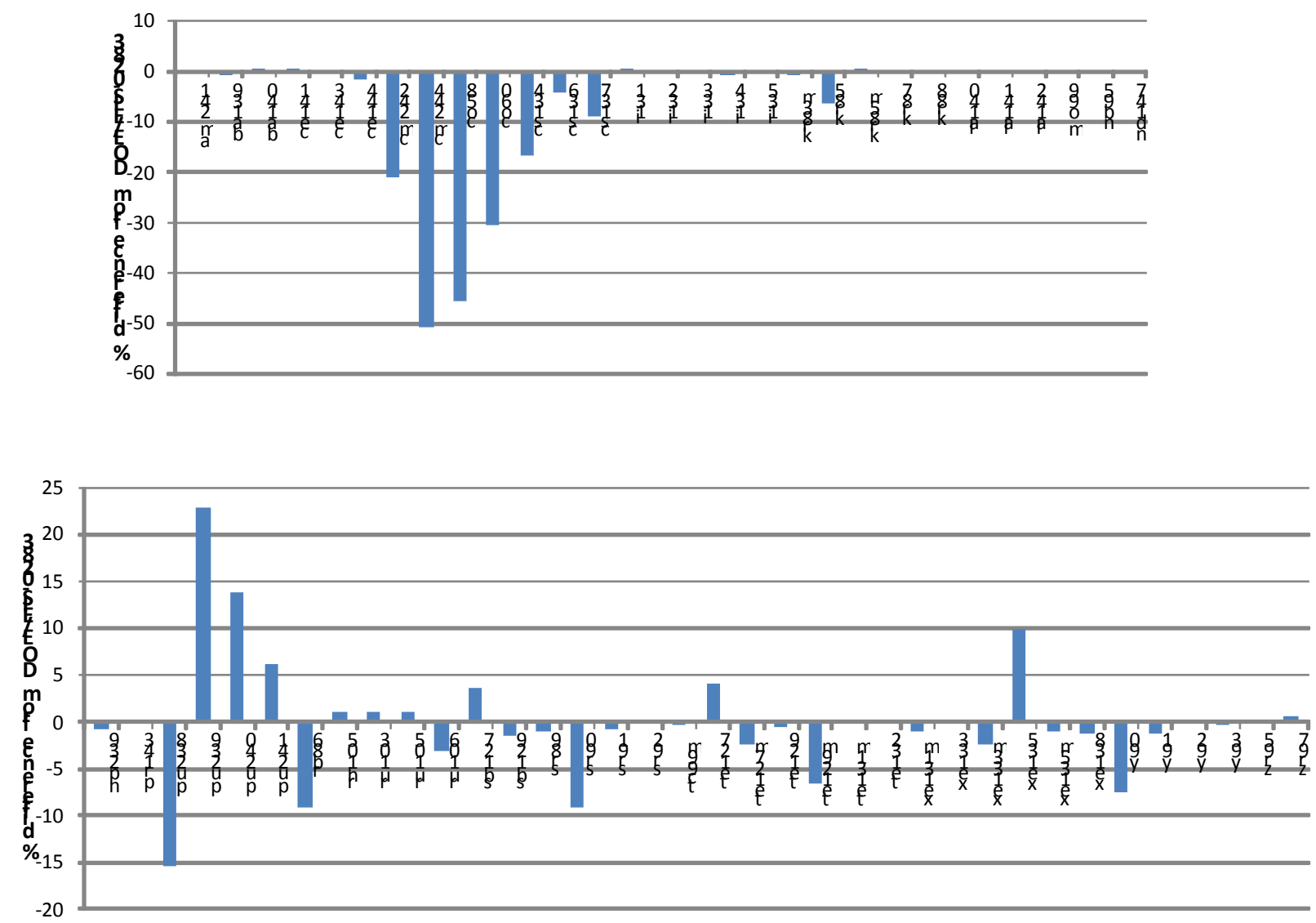

Figure 12. SQN \% differences of MOX/LEU nuclide ratios from DOE/EIS-0283 values.

BFN 
Table 8 presents a summary of the total mass of each of the considered nuclides in the BFN MOX and LEU cores as well as the ratios of the nuclide contents in the MOX core and the LEU core, respectively. The total isotopic masses were determined by multiplying the core-weighted-average isotopic mass per MTHM by the number of tonnes in each core (137.00 MTHM in MOX core, 138.67 MTHM in LEU core). 
Table 8. Summary of nuclide concentrations for the BFN equilibrium MOX and LEU cores

\begin{tabular}{|c|c|c|c|c|c|c|c|}
\hline \multirow{4}{*}{ Nuclide } & \multicolumn{4}{|c|}{ MOX core } & \multicolumn{2}{|c|}{ LEU core } & \multirow{4}{*}{ Ratios } \\
\hline & \multicolumn{3}{|c|}{ Core-weighted-average components } & \multirow[b]{2}{*}{ Total mass $^{a}$} & \multirow{2}{*}{$\begin{array}{c}\text { Core-weighted- } \\
\text { average of LEU } \\
\text { assemblies }\end{array}$} & \multirow[b]{2}{*}{ Total mass ${ }^{a}$} & \\
\hline & $\begin{array}{c}\text { MOX } \\
\text { assemblies }\end{array}$ & $\begin{array}{c}\text { LEU } \\
\text { assemblies }\end{array}$ & $\begin{array}{l}\text { MOX+LEU } \\
\text { assemblies }\end{array}$ & & & & \\
\hline & (g/MTHM) & (g/MTHM) & (g/MTHM) & (g) & (g/MTHM) & (g) & \\
\hline $\operatorname{am} 241$ & $5.2603 \mathrm{E}+01$ & $2.1374 \mathrm{E}+01$ & $7.3977 \mathrm{E}+01$ & $1.0135 \mathrm{E}+04$ & $3.8521 \mathrm{E}+01$ & $5.3417 \mathrm{E}+03$ & 1.9204 \\
\hline $\mathrm{am} 242$ & $8.8702 \mathrm{E}-02$ & $4.4986 \mathrm{E}-02$ & $1.3369 \mathrm{E}-01$ & $1.8315 \mathrm{E}+01$ & $8.3278 \mathrm{E}-02$ & $1.1548 \mathrm{E}+01$ & 1.6053 \\
\hline $\mathrm{am} 242 \mathrm{~m}$ & $7.0833 \mathrm{E}-01$ & $2.7508 \mathrm{E}-01$ & $9.8341 \mathrm{E}-01$ & $1.3473 \mathrm{E}+02$ & $4.9651 \mathrm{E}-01$ & $6.8851 \mathrm{E}+01$ & 1.9807 \\
\hline $\mathrm{am} 243$ & $7.9083 \mathrm{E}+01$ & $5.0140 \mathrm{E}+01$ & $1.2922 \mathrm{E}+02$ & $1.7704 \mathrm{E}+04$ & $1.0139 \mathrm{E}+02$ & $1.4060 \mathrm{E}+04$ & 1.2745 \\
\hline $\mathrm{am} 244$ & $3.2949 \mathrm{E}-03$ & $2.2371 \mathrm{E}-03$ & $5.5320 \mathrm{E}-03$ & 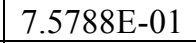 & $4.5720 \mathrm{E}-03$ & $6.3399 \mathrm{E}-01$ & 1.2100 \\
\hline $\operatorname{am} 245$ & $9.3161 \mathrm{E}-07$ & $7.4226 \mathrm{E}-07$ & $1.6739 \mathrm{E}-06$ & \begin{tabular}{|l|}
$2.2932 \mathrm{E}-04$ \\
\end{tabular} & $1.5506 \mathrm{E}-06$ & $2.1502 \mathrm{E}-04$ & 1.0795 \\
\hline ba $137 \mathrm{~m}$ & $8.1237 \mathrm{E}-05$ & $1.0982 \mathrm{E}-04$ & $1.9106 \mathrm{E}-04$ & $2.6175 \mathrm{E}-02$ & $2.0549 \mathrm{E}-04$ & $2.8495 \mathrm{E}-02$ & 0.9298 \\
\hline ba139 & $3.7098 \mathrm{E}-02$ & 4.8494E-02 & $8.5592 \mathrm{E}-02$ & $1.1726 \mathrm{E}+01$ & $8.7061 \mathrm{E}-02$ & $1.2073 \mathrm{E}+01$ & 0.9831 \\
\hline ba140 & $7.9257 \mathrm{E}+00$ & $1.0432 \mathrm{E}+01$ & $1.8358 \mathrm{E}+01$ & $2.5151 \mathrm{E}+03$ & $1.8722 \mathrm{E}+01$ & $2.5961 \mathrm{E}+03$ & 0.9806 \\
\hline ba141 & $7.3904 \mathrm{E}-03$ & $9.7545 \mathrm{E}-03$ & $1.7145 \mathrm{E}-02$ & $2.3488 \mathrm{E}+00$ & 1.7499E-02 & $2.4266 \mathrm{E}+00$ & 0.9797 \\
\hline ba142 & $3.9761 \mathrm{E}-03$ & $5.3737 \mathrm{E}-03$ & $9.3498 \mathrm{E}-03$ & $1.2809 \mathrm{E}+00$ & $9.6311 \mathrm{E}-03$ & $1.3355 \mathrm{E}+00$ & 0.9708 \\
\hline br83 & $2.1949 \mathrm{E}-03$ & $3.4307 \mathrm{E}-03$ & $5.6256 \mathrm{E}-03$ & 7.7070E-01 & $6.1026 \mathrm{E}$ & $8.4624 \mathrm{E}-01$ & 0.9218 \\
\hline br84 & $7.9773 \mathrm{E}-04$ & $1.3019 \mathrm{E}-03$ & $2.0996 \mathrm{E}-03$ & $2.8765 \mathrm{E}-01$ & $2.3120 \mathrm{E}-03$ & $3.2060 \mathrm{E}-01$ & 0.9081 \\
\hline br84m & $9.2891 \mathrm{E}-06$ & $9.6909 \mathrm{E}-06$ & $1.8980 \mathrm{E}-05$ & $2.6003 \mathrm{E}-03$ & $1.7601 \mathrm{E}-05$ & $2.4407 \mathrm{E}-03$ & 1.0783 \\
\hline br85 & $9.4292 \mathrm{E}-05$ & $1.6220 \mathrm{E}-04$ & $2.5650 \mathrm{E}-04$ & $3.5140 \mathrm{E}-02$ & $2.8727 \mathrm{E}-04$ & $3.9835 \mathrm{E}-02$ & 0.8929 \\
\hline br87 & $4.3931 \mathrm{E}-05$ & 7.7779E-05 & $1.2171 \mathrm{E}-04$ & $1.6674 \mathrm{E}-02$ & $1.3767 \mathrm{E}-04$ & $1.9091 \mathrm{E}-02$ & 0.8841 \\
\hline cd112 & $8.7109 \mathrm{E}+00$ & $6.2509 \mathrm{E}+00$ & $1.4962 \mathrm{E}+01$ & $2.0498 \mathrm{E}+03$ & $1.1975 \mathrm{E}+01$ & $1.6605 \mathrm{E}+03$ & 1.2495 \\
\hline cd114 & $9.7000 \mathrm{E}+00$ & $7.8800 \mathrm{E}+00$ & $1.7580 \mathrm{E}+01$ & $2.4085 \mathrm{E}+03$ & $1.4999 \mathrm{E}+01$ & $2.0799 \mathrm{E}+03$ & 1.1721 \\
\hline ce140 & $4.8596 \mathrm{E}+02$ & $7.2799 \mathrm{E}+02$ & $1.2139 \mathrm{E}+03$ & $1.6631 \mathrm{E}+05$ & $1.3654 \mathrm{E}+03$ & $1.8934 \mathrm{E}+05$ & 0.8891 \\
\hline ce141 & $1.9163 \mathrm{E}+01$ & $2.5230 \mathrm{E}+01$ & $4.4394 \mathrm{E}+01$ & $6.0819 \mathrm{E}+03$ & $4.5290 \mathrm{E}+01$ & $6.2803 \mathrm{E}+03$ & 0.9802 \\
\hline ce142 & $4.4660 \mathrm{E}+02$ & $6.7742 \mathrm{E}+02$ & $1.1240 \mathrm{E}+03$ & $1.5399 \mathrm{E}+05$ & $1.2668 \mathrm{E}+03$ & $1.7567 \mathrm{E}+05$ & 0.8873 \\
\hline ce143 & $7.3392 \mathrm{E}-01$ & $1.0159 \mathrm{E}+00$ & $1.7498 \mathrm{E}+00$ & $2.3972 \mathrm{E}+02$ & $1.8183 \mathrm{E}+00$ & $2.5214 \mathrm{E}+02$ & 0.9624 \\
\hline ce144 & $1.2431 \mathrm{E}+02$ & $1.8473 \mathrm{E}+02$ & $3.0904 \mathrm{E}+02$ & $4.2339 \mathrm{E}+04$ & $3.3262 \mathrm{E}+02$ & $4.6125 \mathrm{E}+04$ & 0.9291 \\
\hline ce145 & $7.7893 \mathrm{E}-04$ & $1.0627 \mathrm{E}-03$ & $1.8416 \mathrm{E}-03$ & $2.5230 \mathrm{E}-01$ & $1.9037 \mathrm{E}-03$ & $2.6399 \mathrm{E}-01$ & 0.9674 \\
\hline $\mathrm{cm} 242$ & $1.3315 \mathrm{E}+01$ & $6.5644 \mathrm{E}+00$ & $1.9879 \mathrm{E}+01$ & $2.7235 \mathrm{E}+03$ & $1.2865 \mathrm{E}+01$ & $1.7839 \mathrm{E}+03$ & 1.5453 \\
\hline $\mathrm{cm} 243$ & $3.2214 \mathrm{E}-01$ & $1.5148 \mathrm{E}-01$ & $4.7362 \mathrm{E}-01$ & $6.4886 \mathrm{E}+01$ & $3.0569 \mathrm{E}-01$ & $4.2390 \mathrm{E}+01$ & 1.5494 \\
\hline $\mathrm{cm} 244$ & $2.7704 \mathrm{E}+01$ & $1.8041 \mathrm{E}+01$ & $4.5745 \mathrm{E}+01$ & $6.2671 \mathrm{E}+03$ & $3.7766 \mathrm{E}+01$ & $5.2370 \mathrm{E}+03$ & 1.2113 \\
\hline $\mathrm{cm} 245$ & $1.7595 \mathrm{E}+00$ & $9.1022 \mathrm{E}-01$ & $2.6697 \mathrm{E}+00$ & $3.6575 \mathrm{E}+02$ & $1.9531 \mathrm{E}+00$ & $2.7084 \mathrm{E}+02$ & 1.3669 \\
\hline $\mathrm{cm} 246$ & $2.1005 \mathrm{E}-01$ & $1.2748 \mathrm{E}-01$ & $3.3753 \mathrm{E}-01$ & $4.6241 \mathrm{E}+01$ & $2.8514 \mathrm{E}-01$ & $3.9540 \mathrm{E}+01$ & 1.1837 \\
\hline $\operatorname{co5} 5$ & $4.4325 \mathrm{E}-18$ & $2.8874 \mathrm{E}-18$ & $7.3199 \mathrm{E}-18$ & $1.0028 \mathrm{E}-15$ & $5.7435 \mathrm{E}-18$ & $7.9646 \mathrm{E}-16$ & 1.2745 \\
\hline co60 & $3.9713 \mathrm{E}-15$ & $5.8200 \mathrm{E}-15$ & $9.7914 \mathrm{E}-15$ & $1.3414 \mathrm{E}-12$ & $1.0913 \mathrm{E}-14$ & $1.5133 \mathrm{E}-12$ & 0.8972 \\
\hline $\operatorname{cs} 133$ & $4.9492 \mathrm{E}+02$ & $6.7293 \mathrm{E}+02$ & $1.1678 \mathrm{E}+03$ & $1.6000 \mathrm{E}+05$ & $1.2530 \mathrm{E}+03$ & $1.7375 \mathrm{E}+05$ & 0.9320 \\
\hline cs134 & $4.5495 \mathrm{E}+01$ & $6.4332 \mathrm{E}+01$ & $1.0983 \mathrm{E}+02$ & $1.5046 \mathrm{E}+04$ & $1.2371 \mathrm{E}+02$ & $1.7155 \mathrm{E}+04$ & 0.8878 \\
\hline cs135 & $2.5167 \mathrm{E}+02$ & $2.7667 \mathrm{E}+02$ & $5.2834 \mathrm{E}+02$ & $7.2382 \mathrm{E}+04$ & $5.1289 \mathrm{E}+02$ & $7.1122 \mathrm{E}+04$ & 1.0301 \\
\hline $\operatorname{cs} 136$ & $2.5114 \mathrm{E}-01$ & $2.7618 \mathrm{E}-01$ & $5.2733 \mathrm{E}-01$ & $7.2244 \mathrm{E}+01$ & & $7.2181 \mathrm{E}+01$ & \\
\hline $\operatorname{cs} 137$ & $5.2924 \mathrm{E}+02$ & $7.1719 \mathrm{E}+02$ & $1.2464 \mathrm{E}+03$ & $1.7076 \mathrm{E}+05$ & $1.3418 \mathrm{E}+03$ & $1.8607 \mathrm{E}+05$ & 0.9289 \\
\hline cs138 & $1.5570 \mathrm{E}-02$ & $2.0310 \mathrm{E}-02$ & $3.5879 \mathrm{E}-02$ & $4.9155 \mathrm{E}+00$ & $3.6466 \mathrm{E}-02$ & $5.0568 \mathrm{E}+00$ & 0.9839 \\
\hline cs139 & $4.0318 \mathrm{E}-03$ & $5.2978 \mathrm{E}-03$ & $9.3296 \mathrm{E}-03$ & $1.2782 \mathrm{E}+00$ & $9.5080 \mathrm{E}-03$ & $1.3185 \mathrm{E}+00$ & 0.9812 \\
\hline $\operatorname{cs} 140$ & $3.7757 \mathrm{E}-04$ & $5.1775 \mathrm{E}-04$ & $8.9533 \mathrm{E}-04$ & $1.2266 \mathrm{E}-01$ & $9.2749 \mathrm{E}-04$ & $1.2862 \mathrm{E}-01$ & 0.9653 \\
\hline $\operatorname{cs} 141$ & $1.2068 \mathrm{E}-04$ & $1.5934 \mathrm{E}-04$ & $2.8001 \mathrm{E}-04$ & $3.8362 \mathrm{E}-02$ & $2.8602 \mathrm{E}-04$ & $3.9662 \mathrm{E}-02$ & 0.9790 \\
\hline eu151 & $1.3950 \mathrm{E}-02$ & $9.9766 \mathrm{E}-03$ & $2.3927 \mathrm{E}-02$ & $3.2780 \mathrm{E}+00$ & $1.7396 \mathrm{E}-02$ & $2.4122 \mathrm{E}+00$ & 1.3755 \\
\hline eu153 & $5.9751 \mathrm{E}+01$ & $6.4757 \mathrm{E}+01$ & $1.2451 \mathrm{E}+02$ & $1.7058 \mathrm{E}+04$ & $1.2222 \mathrm{E}+02$ & $1.6949 \mathrm{E}+04$ & 1.0187 \\
\hline eu154 & $1.2690 \mathrm{E}+01$ & $1.2492 \mathrm{E}+01$ & $2.5182 \mathrm{E}+01$ & $3.4499 \mathrm{E}+03$ & $2.3821 \mathrm{E}+01$ & $3.3033 \mathrm{E}+03$ & 1.0571 \\
\hline eu155 & $4.3371 \mathrm{E}+00$ & $4.4466 \mathrm{E}+00$ & $8.7837 \mathrm{E}+00$ & $1.2034 \mathrm{E}+03$ & $8.4894 \mathrm{E}+00$ & $1.1772 \mathrm{E}+03$ & 1.0347 \\
\hline $\operatorname{gd} 154$ & $7.1885 \mathrm{E}+01$ & $1.3033 \mathrm{E}+02$ & $2.0222 \mathrm{E}+02$ & $2.7704 \mathrm{E}+04$ & $2.2476 \mathrm{E}+02$ & $3.1167 \mathrm{E}+04$ & 0.8997 \\
\hline
\end{tabular}


Table 8 (continued)

\begin{tabular}{|c|c|c|c|c|c|c|c|}
\hline \multirow{4}{*}{ Nuclide } & \multicolumn{4}{|c|}{ MOX core } & \multicolumn{2}{|c|}{ LEU core } & \multirow{4}{*}{ Ratios } \\
\hline & \multicolumn{3}{|c|}{ Core-weighted-average components } & \multirow[b]{2}{*}{ Total mass ${ }^{a}$} & \multirow{2}{*}{$\begin{array}{c}\text { Core-weighted } \\
\text { average of LEU } \\
\text { assemblies } \\
\end{array}$} & \multirow[b]{2}{*}{ Total mass ${ }^{a}$} & \\
\hline & $\begin{array}{c}\text { MOX } \\
\text { assemblies }\end{array}$ & \begin{tabular}{c|c|} 
LEU \\
assemblies
\end{tabular} & $\begin{array}{c}\text { MOX+LEU } \\
\text { assemblies }\end{array}$ & & & & \\
\hline & (g/MTHM) & (g/MTHM) & (g/MTHM) & (g) & (g/MTHM) & (g) & \\
\hline $\operatorname{gd} 155$ & $1.7776 \mathrm{E}+00$ & $-5.9321 \mathrm{E}-01$ & $1.1844 \mathrm{E}+00$ & $1.6226 \mathrm{E}+02$ & $2.6641 \mathrm{E}+00$ & $3.6943 \mathrm{E}+02$ & 0.4446 \\
\hline $\operatorname{gd} 156$ & $1.3487 \mathrm{E}+03$ & $2.4527 \mathrm{E}+03$ & $3.8014 \mathrm{E}+03$ & $5.2080 \mathrm{E}+05$ & $4.2674 \mathrm{E}+03$ & $5.9176 \mathrm{E}+05$ & 0.8908 \\
\hline $\operatorname{gd} 157$ & $4.9087 \mathrm{E}-01$ & $5.0099 \mathrm{E}-01$ & $9.9186 \mathrm{E}-01$ & $1.3588 \mathrm{E}+02$ & $9.1033 \mathrm{E}-01$ & $1.2624 \mathrm{E}+02$ & 1.0896 \\
\hline $\operatorname{gd} 158$ & $1.5922 \mathrm{E}+03$ & $2.8955 \mathrm{E}+03$ & $4.4877 \mathrm{E}+03$ & $6.1482 \mathrm{E}+05$ & $5.0379 \mathrm{E}+03$ & $6.9860 \mathrm{E}+05$ & 0.8908 \\
\hline $\mathrm{i} 129$ & $8.8725 \mathrm{E}+01$ & $8.7245 \mathrm{E}+01$ & $1.7597 \mathrm{E}+02$ & $2.4108 \mathrm{E}+04$ & $1.6422 \mathrm{E}+02$ & $2.2772 \mathrm{E}+04$ & 1.0716 \\
\hline i130 & $3.8230 \mathrm{E}-03$ & $4.3250 \mathrm{E}-03$ & $8.1480 \mathrm{E}-03$ & $1.1163 \mathrm{E}+00$ & $8.3406 \mathrm{E}-03$ & $1.1566 \mathrm{E}+00$ & 0.9769 \\
\hline i131 & $2.9091 \mathrm{E}+00$ & $3.4693 \mathrm{E}+00$ & $6.3784 \mathrm{E}+00$ & $8.7384 \mathrm{E}+02$ & $6.2565 \mathrm{E}+00$ & $8.6758 \mathrm{E}+02$ & 1.0195 \\
\hline i132 & $5.0342 \mathrm{E}-02$ & 6.0943E-02 & $1.1129 \mathrm{E}-01$ & $1.5246 \mathrm{E}+01$ & $1.0985 \mathrm{E}-01$ & $1.5234 \mathrm{E}+01$ & 1.0130 \\
\hline i133 & $6.2245 \mathrm{E}-01$ & $7.8404 \mathrm{E}-01$ & $1.4065 \mathrm{E}+00$ & $1.9269 \mathrm{E}+02$ & $1.4099 \mathrm{E}+00$ & $1.9551 \mathrm{E}+02$ & 0.9976 \\
\hline $\mathrm{i} 134$ & $2.9091 \mathrm{E}-02$ & 3.7337E-02 & $6.6427 \mathrm{E}-02$ & $9.1006 \mathrm{E}+00$ & $6.7094 \mathrm{E}-02$ & $9.3039 \mathrm{E}+00$ & 0.9901 \\
\hline $\mathrm{i} 135$ & $1.9161 \mathrm{E}-01$ & $2.3933 \mathrm{E}-01$ & 4.3094E-01 & $5.9039 \mathrm{E}+01$ & $4.3076 \mathrm{E}-01$ & $5.9733 \mathrm{E}+01$ & 1.0004 \\
\hline $\mathrm{i} 136 \mathrm{~m}$ & $9.3852 \mathrm{E}-05$ & $1.0895 \mathrm{E}-04$ & $2.0280 \mathrm{E}-04$ & \begin{tabular}{|l|}
$2.7784 \mathrm{E}-02$ \\
\end{tabular} & $1.9694 \mathrm{E}-04$ & $2.7310 \mathrm{E}-02$ & 1.0298 \\
\hline kr83m & $1.6622 \mathrm{E}-03$ & $2.5984 \mathrm{E}-03$ & $4.2606 \mathrm{E}-03$ & 5.8370E-01 & $4.6236 \mathrm{E}-03$ & $6.4116 \mathrm{E}-01$ & 0.9215 \\
\hline kr85 & $8.9377 \mathrm{E}+00$ & $1.8858 \mathrm{E}+01$ & $2.7796 \mathrm{E}+01$ & $3.8080 \mathrm{E}+03$ & $3.4888 \mathrm{E}+01$ & $4.8379 \mathrm{E}+03$ & 0.7967 \\
\hline $\mathrm{kr} 85 \mathrm{~m}$ & $8.4324 \mathrm{E}-03$ & $1.4512 \mathrm{E}-02$ & $2.2944 \mathrm{E}-02$ & $3.1433 \mathrm{E}+00$ & $2.5707 \mathrm{E}-02$ & $3.5648 \mathrm{E}+00$ & 0.8925 \\
\hline kr87 & $4.6699 \mathrm{E}-03$ & $8.1054 \mathrm{E}-03$ & $1.2775 \mathrm{E}-02$ & $1.7502 \mathrm{E}+00$ & $1.4352 \mathrm{E}-02$ & $1.9902 \mathrm{E}+00$ & 0.8902 \\
\hline $\mathrm{kr} 88$ & $1.3750 \mathrm{E}-02$ & $2.4313 \mathrm{E}-02$ & $3.8063 \mathrm{E}-02$ & $5.2147 \mathrm{E}+00$ & 4.3019E-02 & $5.9655 \mathrm{E}+00$ & 0.8848 \\
\hline kr89 & $3.1275 \mathrm{E}-04$ & $5.6934 \mathrm{E}-04$ & $8.8208 \mathrm{E}-04$ & $1.2085 \mathrm{E}-01$ & $1.0061 \mathrm{E}-03$ & $1.3951 \mathrm{E}-01$ & 0.8767 \\
\hline kr90 & $5.3914 \mathrm{E}-05$ & $1.0259 \mathrm{E}-04$ & $1.5651 \mathrm{E}-04$ & $2.1442 \mathrm{E}-02$ & 1.8103E-04 & $2.5103 \mathrm{E}-02$ & 0.8646 \\
\hline la139 & $4.9730 \mathrm{E}+02$ & $7.3189 \mathrm{E}+02$ & $1.2292 \mathrm{E}+03$ & $1.6840 \mathrm{E}+05$ & $1.3687 \mathrm{E}+03$ & $1.8980 \mathrm{E}+05$ & 0.8981 \\
\hline la140 & $1.0808 \mathrm{E}+00$ & $1.4282 \mathrm{E}+00$ & $2.5090 \mathrm{E}+00$ & $3.4374 \mathrm{E}+02$ & $2.5714 \mathrm{E}+00$ & $3.5658 \mathrm{E}+02$ & 0.9757 \\
\hline la141 & $9.5970 \mathrm{E}-02$ & $1.2640 \mathrm{E}-01$ & $2.2237 \mathrm{E}-01$ & $3.0465 \mathrm{E}+01$ & $2.2677 \mathrm{E}-01$ & $3.1446 \mathrm{E}+01$ & 0.9806 \\
\hline la142 & $3.5544 \mathrm{E}-02$ & 4.7632E-02 & $8.3176 \mathrm{E}-02$ & $1.1395 \mathrm{E}+01$ & $8.5373 \mathrm{E}-02$ & $1.1839 \mathrm{E}+01$ & 0.9743 \\
\hline la143 & $5.2223 \mathrm{E}-03$ & $7.2202 \mathrm{E}-03$ & $1.2443 \mathrm{E}-02$ & $1.7046 \mathrm{E}+00$ & $1.2924 \mathrm{E}-02$ & $1.7921 \mathrm{E}+00$ & 0.9628 \\
\hline mo100 & $4.0885 \mathrm{E}+02$ & $5.5658 \mathrm{E}+02$ & $9.6543 \mathrm{E}+02$ & $1.3226 \mathrm{E}+05$ & $1.0428 \mathrm{E}+03$ & $1.4460 \mathrm{E}+05$ & 0.9258 \\
\hline mo92 & $2.1739 \mathrm{E}-12$ & $3.7620 \mathrm{E}-13$ & $2.5501 \mathrm{E}-12$ & 3.4936E-10 & $7.6605 \mathrm{E}-13$ & $1.0623 \mathrm{E}-10$ & 3.3289 \\
\hline mo94 & $3.7144 \mathrm{E}-03$ & $5.7401 \mathrm{E}-03$ & $9.4545 \mathrm{E}-03$ & $1.2953 \mathrm{E}+00$ & $1.1334 \mathrm{E}-02$ & $1.5716 \mathrm{E}+00$ & 0.8342 \\
\hline mo95 & $2.4872 \mathrm{E}+02$ & $4.0807 \mathrm{E}+02$ & $6.5679 \mathrm{E}+02$ & $8.9980 \mathrm{E}+04$ & $7.6397 \mathrm{E}+02$ & $1.0594 \mathrm{E}+05$ & 0.8597 \\
\hline mo96 & $1.2387 \mathrm{E}+01$ & $2.2699 \mathrm{E}+01$ & $3.5086 \mathrm{E}+01$ & $4.8067 \mathrm{E}+03$ & $4.4642 \mathrm{E}+01$ & $6.1905 \mathrm{E}+03$ & 0.7859 \\
\hline mo97 & $3.2889 \mathrm{E}+02$ & $4.8467 \mathrm{E}+02$ & $8.1356 \mathrm{E}+02$ & $1.1146 \mathrm{E}+05$ & $9.0643 \mathrm{E}+02$ & $1.2569 \mathrm{E}+05$ & 0.8975 \\
\hline mo98 & $3.4919 \mathrm{E}+02$ & $4.9209 \mathrm{E}+02$ & $8.4128 \mathrm{E}+02$ & $1.1526 \mathrm{E}+05$ & $9.2170 \mathrm{E}+02$ & $1.2781 \mathrm{E}+05$ & 0.9127 \\
\hline mo99 & $1.3221 \mathrm{E}+00$ & $1.6769 \mathrm{E}+00$ & $2.9990 \mathrm{E}+00$ & $4.1086 \mathrm{E}+02$ & $3.0162 \mathrm{E}+00$ & $4.1826 \mathrm{E}+02$ & 0.9943 \\
\hline nb95 & $1.3273 \mathrm{E}+01$ & $1.8828 \mathrm{E}+01$ & $3.2101 \mathrm{E}+01$ & $4.3978 \mathrm{E}+03$ & $3.3691 \mathrm{E}+01$ & $4.6719 \mathrm{E}+03$ & 0.9528 \\
\hline nb97 & $2.0997 \mathrm{E}-02$ & $2.7723 \mathrm{E}-02$ & $4.8721 \mathrm{E}-02$ & $6.6747 \mathrm{E}+00$ & 4.9742E-02 & $6.8977 \mathrm{E}+00$ & 0.9795 \\
\hline nb97m & $4.2725 \mathrm{E}-07$ & $4.0498 \mathrm{E}-07$ & $8.3223 \mathrm{E}-07$ & $1.1402 \mathrm{E}-04$ & $7.3950 \mathrm{E}-07$ & $1.0255 \mathrm{E}-04$ & 1.1254 \\
\hline nd142 & $6.6077 \mathrm{E}+00$ & $1.2061 \mathrm{E}+01$ & $1.8668 \mathrm{E}+01$ & $2.5576 \mathrm{E}+03$ & $2.3814 \mathrm{E}+01$ & $3.3023 \mathrm{E}+03$ & 0.7839 \\
\hline nd143 & $3.2077 \mathrm{E}+02$ & $4.7401 \mathrm{E}+02$ & $7.9478 \mathrm{E}+02$ & $1.0889 \mathrm{E}+05$ & $8.6990 \mathrm{E}+02$ & $1.2063 \mathrm{E}+05$ & 0.9136 \\
\hline nd144 & $3.3804 \mathrm{E}+02$ & $6.1566 \mathrm{E}+02$ & $9.5370 \mathrm{E}+02$ & $1.3066 \mathrm{E}+05$ & $1.1788 \mathrm{E}+03$ & $1.6347 \mathrm{E}+05$ & 0.8090 \\
\hline nd145 & $2.6227 \mathrm{E}+02$ & $4.0556 \mathrm{E}+02$ & $6.6783 \mathrm{E}+02$ & $9.1493 \mathrm{E}+04$ & $7.5358 \mathrm{E}+02$ & $1.0450 \mathrm{E}+05$ & 0.8862 \\
\hline nd146 & $2.7112 \mathrm{E}+02$ & $4.2043 \mathrm{E}+02$ & $6.9156 \mathrm{E}+02$ & $9.4743 \mathrm{E}+04$ & $7.9115 \mathrm{E}+02$ & $1.0971 \mathrm{E}+05$ & 0.8741 \\
\hline nd147 & $2.7168 \mathrm{E}+00$ & $3.5162 \mathrm{E}+00$ & $6.2330 \mathrm{E}+00$ & $8.5392 \mathrm{E}+02$ & $6.3202 \mathrm{E}+00$ & $8.7642 \mathrm{E}+02$ & 0.9862 \\
\hline nd148 & $1.5755 \mathrm{E}+02$ & $2.2034 \mathrm{E}+02$ & $3.7789 \mathrm{E}+02$ & $5.1772 \mathrm{E}+04$ & $4.1269 \mathrm{E}+02$ & $5.7228 \mathrm{E}+04$ & 0.9157 \\
\hline nd150 & $8.7902 \mathrm{E}+01$ & $1.0301 \mathrm{E}+02$ & $1.9091 \mathrm{E}+02$ & $2.6155 \mathrm{E}+04$ & $1.9365 \mathrm{E}+02$ & $2.6854 \mathrm{E}+04$ & 0.9858 \\
\hline np237 & $8.8554 \mathrm{E}+01$ & $2.3836 \mathrm{E}+02$ & $3.2691 \mathrm{E}+02$ & $4.4787 \mathrm{E}+04$ & $4.4805 \mathrm{E}+02$ & $6.2131 \mathrm{E}+04$ & 0.7296 \\
\hline np238 & $1.8909 \mathrm{E}-01$ & $5.5154 \mathrm{E}-01$ & $7.4062 \mathrm{E}-01$ & $1.0147 \mathrm{E}+02$ & $1.0576 \mathrm{E}+00$ & $1.4665 \mathrm{E}+02$ & 0.7003 \\
\hline
\end{tabular}


Table 8 (continued)

\begin{tabular}{|c|c|c|c|c|c|c|c|}
\hline \multirow{4}{*}{ Nuclide } & \multicolumn{4}{|c|}{ MOX core } & \multicolumn{2}{|c|}{ LEU core } & \multirow{4}{*}{ Ratios } \\
\hline & \multicolumn{3}{|c|}{ Core-weighted-average components } & \multirow[b]{2}{*}{ Total mass ${ }^{a}$} & \multirow{2}{*}{\begin{tabular}{|c|} 
Core-weighted \\
average of LEU \\
assemblies
\end{tabular}} & \multirow[b]{2}{*}{ Total mass ${ }^{a}$} & \\
\hline & $\begin{array}{c}\text { MOX } \\
\text { assemblies }\end{array}$ & \begin{tabular}{c|c|} 
LEU \\
assemblies
\end{tabular} & $\begin{array}{c}\text { MOX+LEU } \\
\text { assemblies }\end{array}$ & & & & \\
\hline & (g/MTHM) & (g/MTHM) & (g/MTHM) & (g) & (g/MTHM) & (g) & \\
\hline np239 & $2.6209 \mathrm{E}+01$ & $3.2915 \mathrm{E}+01$ & $5.9124 \mathrm{E}+01$ & $8.1000 \mathrm{E}+03$ & $6.0452 \mathrm{E}+01$ & $8.3828 \mathrm{E}+03$ & 0.9780 \\
\hline np240 & $2.3042 \mathrm{E}-04$ & $2.9642 \mathrm{E}-04$ & 5.2684E-04 & 7.2177E-02 & $5.5588 \mathrm{E}-04$ & $7.7084 \mathrm{E}-02$ & 0.9478 \\
\hline pd105 & $2.7603 \mathrm{E}+02$ & $2.1126 \mathrm{E}+02$ & $4.8729 \mathrm{E}+02$ & $6.6759 \mathrm{E}+04$ & $4.0095 \mathrm{E}+02$ & $5.5600 \mathrm{E}+04$ & 1.2153 \\
\hline pd107 & $1.7339 \mathrm{E}+02$ & $1.1079 \mathrm{E}+02$ & $2.8418 \mathrm{E}+02$ & $3.8932 \mathrm{E}+04$ & $2.1244 \mathrm{E}+02$ & $2.9460 \mathrm{E}+04$ & 1.3377 \\
\hline pd108 & $1.1830 \mathrm{E}+02$ & $7.2303 \mathrm{E}+01$ & $1.9060 \mathrm{E}+02$ & $2.6113 \mathrm{E}+04$ & $1.3917 \mathrm{E}+02$ & $1.9298 \mathrm{E}+04$ & 1.3696 \\
\hline pd110 & $3.8356 \mathrm{E}+01$ & $2.3991 \mathrm{E}+01$ & $6.2347 \mathrm{E}+01$ & $8.5416 \mathrm{E}+03$ & $4.6206 \mathrm{E}+01$ & $6.4073 \mathrm{E}+03$ & 1.3493 \\
\hline pm147 & $7.9916 \mathrm{E}+01$ & $1.1105 \mathrm{E}+02$ & $1.9096 \mathrm{E}+02$ & $2.6162 \mathrm{E}+04$ & $2.0119 \mathrm{E}+02$ & $2.7899 \mathrm{E}+04$ & 0.9492 \\
\hline pr143 & $7.1210 \mathrm{E}+00$ & $9.8881 \mathrm{E}+00$ & $1.7009 \mathrm{E}+01$ & $2.3302 \mathrm{E}+03$ & $1.7663 \mathrm{E}+01$ & $2.4493 \mathrm{E}+03$ & 0.9630 \\
\hline pr144 & $5.2607 \mathrm{E}-03$ & $7.8192 \mathrm{E}-03$ & $1.3080 \mathrm{E}-02$ & $1.7919 \mathrm{E}+00$ & $1.4082 \mathrm{E}-02$ & $1.9528 \mathrm{E}+00$ & 0.9288 \\
\hline pr145 & $9.3006 \mathrm{E}-02$ & $1.2683 \mathrm{E}-01$ & 2.1984E-01 & $3.0118 \mathrm{E}+01$ & $2.2723 \mathrm{E}-01$ & $3.1510 \mathrm{E}+01$ & 0.9675 \\
\hline pu237 & $1.2811 \mathrm{E}-04$ & $1.6906 \mathrm{E}-04$ & $2.9717 \mathrm{E}-04$ & $4.0712 \mathrm{E}-02$ & $3.3431 \mathrm{E}-04$ & $4.6359 \mathrm{E}-02$ & 0.8889 \\
\hline pu238 & $4.6780 \mathrm{E}+01$ & $8.2018 \mathrm{E}+01$ & $1.2880 \mathrm{E}+02$ & $1.7645 \mathrm{E}+04$ & $1.6036 \mathrm{E}+02$ & $2.2238 \mathrm{E}+04$ & 0.8032 \\
\hline pu239 & $4.6387 \mathrm{E}+03$ & $2.7180 \mathrm{E}+03$ & $7.3567 \mathrm{E}+03$ & $1.0079 \mathrm{E}+06$ & $4.8744 \mathrm{E}+03$ & $6.7593 \mathrm{E}+05$ & 1.5093 \\
\hline pu240 & $2.6745 \mathrm{E}+03$ & $1.1439 \mathrm{E}+03$ & $3.8184 \mathrm{E}+03$ & $5.2312 \mathrm{E}+05$ & $2.1364 \mathrm{E}+03$ & $2.9625 \mathrm{E}+05$ & 1.7873 \\
\hline pu241 & $1.0848 \mathrm{E}+03$ & $5.6609 \mathrm{E}+02$ & $1.6509 \mathrm{E}+03$ & $2.2617 \mathrm{E}+05$ & $1.0537 \mathrm{E}+03$ & $1.4612 \mathrm{E}+05$ & 1.5667 \\
\hline pu242 & $3.9196 \mathrm{E}+02$ & $2.3681 \mathrm{E}+02$ & $6.2877 \mathrm{E}+02$ & $8.6142 \mathrm{E}+04$ & $4.6668 \mathrm{E}+02$ & $6.4714 \mathrm{E}+04$ & 1.3473 \\
\hline pu243 & $7.1116 \mathrm{E}-02$ & 4.8849E-02 & $1.1996 \mathrm{E}-01$ & $1.6435 \mathrm{E}+01$ & $9.6600 \mathrm{E}-02$ & $1.3396 \mathrm{E}+01$ & 1.2419 \\
\hline pu244 & $1.9123 \mathrm{E}-02$ & $1.2844 \mathrm{E}-02$ & $3.1967 \mathrm{E}-02$ & $4.3795 \mathrm{E}+00$ & $2.6812 \mathrm{E}-02$ & $3.7180 \mathrm{E}+00$ & 1.1923 \\
\hline pu245 & $8.4290 \mathrm{E}-07$ & $5.9575 \mathrm{E}-07$ & $1.4387 \mathrm{E}-06$ & $1.9710 \mathrm{E}-04$ & $1.2594 \mathrm{E}-06$ & $1.7465 \mathrm{E}-04$ & 1.1423 \\
\hline $\mathrm{rb} 86$ & $6.1961 \mathrm{E}-03$ & $1.2866 \mathrm{E}-02$ & $1.9062 \mathrm{E}-02$ & $2.6115 \mathrm{E}+00$ & $2.4375 \mathrm{E}-02$ & $3.3800 \mathrm{E}+00$ & 0.7821 \\
\hline $\mathrm{rb} 88$ & $1.4705 \mathrm{E}-03$ & $2.5784 \mathrm{E}-03$ & $4.0488 \mathrm{E}-03$ & $5.5469 \mathrm{E}-01$ & $4.5638 \mathrm{E}-03$ & $6.3287 \mathrm{E}-01$ & 0.8872 \\
\hline $\mathrm{rb89}$ & $1.6541 \mathrm{E}-03$ & $2.9297 \mathrm{E}-03$ & $4.5838 \mathrm{E}-03$ & $6.2798 \mathrm{E}-01$ & $5.1827 \mathrm{E}-03$ & $7.1868 \mathrm{E}-01$ & 0.8845 \\
\hline rb90 & $2.8049 \mathrm{E}-04$ & $5.2445 \mathrm{E}-04$ & 8.0494E-04 & $1.1028 \mathrm{E}-01$ & $9.2601 \mathrm{E}-04$ & $1.2841 \mathrm{E}-01$ & 0.8693 \\
\hline rb90m & $1.1089 \mathrm{E}-04$ & $1.5719 \mathrm{E}-04$ & $2.6809 \mathrm{E}-04$ & $3.6728 \mathrm{E}-02$ & $2.8058 \mathrm{E}-04$ & $3.8907 \mathrm{E}-02$ & 0.9555 \\
\hline rb91 & $1.3604 \mathrm{E}-04$ & $2.3405 \mathrm{E}-04$ & $3.7010 \mathrm{E}-04$ & 5.0703E-02 & $4.1464 \mathrm{E}-04$ & $5.7498 \mathrm{E}-02$ & 0.8926 \\
\hline rh103m & $1.9585 \mathrm{E}-02$ & $2.0234 \mathrm{E}-02$ & $3.9819 \mathrm{E}-02$ & $5.4551 \mathrm{E}+00$ & $3.6850 \mathrm{E}-02$ & $5.1099 \mathrm{E}+00$ & 1.0806 \\
\hline rh105 & $5.4787 \mathrm{E}-01$ & $4.9235 \mathrm{E}-01$ & $1.0402 \mathrm{E}+00$ & $1.4251 \mathrm{E}+02$ & $9.0504 \mathrm{E}-01$ & $1.2550 \mathrm{E}+02$ & 1.1494 \\
\hline rh105m & $5.5626 \mathrm{E}-05$ & $5.0555 \mathrm{E}-05$ & $1.0618 \mathrm{E}-04$ & $1.4547 \mathrm{E}-02$ & $9.2953 \mathrm{E}-05$ & $1.2890 \mathrm{E}-02$ & 1.1423 \\
\hline rh106 & $9.7651 \mathrm{E}-05$ & 7.4154E-05 & $1.7181 \mathrm{E}-04$ & $2.3537 \mathrm{E}-02$ & $1.3884 \mathrm{E}-04$ & $1.9252 \mathrm{E}-02$ & 1.2375 \\
\hline rh107 & $3.6697 \mathrm{E}-03$ & $3.0121 \mathrm{E}-03$ & $6.6818 \mathrm{E}-03$ & $9.1540 \mathrm{E}-01$ & $5.5848 \mathrm{E}-03$ & $7.7445 \mathrm{E}-01$ & 1.1964 \\
\hline ru101 & $3.5366 \mathrm{E}+02$ & $4.6096 \mathrm{E}+02$ & $8.1462 \mathrm{E}+02$ & $1.1160 \mathrm{E}+05$ & $8.6287 \mathrm{E}+02$ & $1.1965 \mathrm{E}+05$ & 0.9441 \\
\hline ru102 & $3.8465 \mathrm{E}+02$ & $4.6312 \mathrm{E}+02$ & $8.4777 \mathrm{E}+02$ & $1.1614 \mathrm{E}+05$ & $8.7205 \mathrm{E}+02$ & $1.2093 \mathrm{E}+05$ & 0.9722 \\
\hline ru103 & $1.9958 \mathrm{E}+01$ & $2.0618 \mathrm{E}+01$ & $4.0576 \mathrm{E}+01$ & $5.5589 \mathrm{E}+03$ & $3.7552 \mathrm{E}+01$ & $5.2073 \mathrm{E}+03$ & 1.0805 \\
\hline ru104 & $3.4229 \mathrm{E}+02$ & $3.0510 \mathrm{E}+02$ & $6.4738 \mathrm{E}+02$ & $8.8692 \mathrm{E}+04$ & $5.7804 \mathrm{E}+02$ & $8.0157 \mathrm{E}+04$ & 1.1200 \\
\hline ru105 & $7.3526 \mathrm{E}-02$ & $6.6824 \mathrm{E}-02$ & $1.4035 \mathrm{E}-01$ & $1.9228 \mathrm{E}+01$ & $1.2288 \mathrm{E}-01$ & $1.7039 \mathrm{E}+01$ & 1.1422 \\
\hline ru106 & $9.8207 \mathrm{E}+01$ & $7.2287 \mathrm{E}+01$ & $1.7049 \mathrm{E}+02$ & $2.3358 \mathrm{E}+04$ & $1.3521 \mathrm{E}+02$ & $1.8750 \mathrm{E}+04$ & 1.2610 \\
\hline ru107 & $6.2471 \mathrm{E}-04$ & $5.1258 \mathrm{E}-04$ & $1.1373 \mathrm{E}-03$ & $1.5581 \mathrm{E}-01$ & $9.5049 \mathrm{E}-04$ & $1.3180 \mathrm{E}-01$ & 1.1965 \\
\hline sb125 & $4.9491 \mathrm{E}+00$ & $4.6088 \mathrm{E}+00$ & $9.5579 \mathrm{E}+00$ & $1.3094 \mathrm{E}+03$ & $8.5818 \mathrm{E}+00$ & $1.1900 \mathrm{E}+03$ & 1.1137 \\
\hline sb127 & $1.4642 \mathrm{E}-01$ & $1.4874 \mathrm{E}-01$ & $2.9516 \mathrm{E}-01$ & $4.0437 \mathrm{E}+01$ & $2.7085 \mathrm{E}-01$ & $3.7559 \mathrm{E}+01$ & 1.0898 \\
\hline $\mathrm{sb} 129$ & $2.0749 \mathrm{E}-02$ & $2.1904 \mathrm{E}-02$ & 4.2654E-02 & $5.8436 \mathrm{E}+00$ & $3.9805 \mathrm{E}-02$ & $5.5197 \mathrm{E}+00$ & 1.0716 \\
\hline sb130 & $2.8105 \mathrm{E}-03$ & 3.3397E-03 & $6.1502 \mathrm{E}-03$ & $8.4258 \mathrm{E}-01$ & $6.0265 \mathrm{E}-03$ & $8.3570 \mathrm{E}-01$ & 1.0205 \\
\hline sb130m & $4.5466 \mathrm{E}-04$ & $5.6433 \mathrm{E}-04$ & $1.0190 \mathrm{E}-03$ & $1.3960 \mathrm{E}-01$ & $1.0156 \mathrm{E}-03$ & $1.4083 \mathrm{E}-01$ & 1.0033 \\
\hline sb133 & $3.4010 \mathrm{E}-04$ & $4.7835 \mathrm{E}-04$ & $8.1845 \mathrm{E}-04$ & 1.1213E-01 & $8.5673 \mathrm{E}-04$ & $1.1880 \mathrm{E}-01$ & 0.9553 \\
\hline se84 & 7.4863E-05 & $1.2338 \mathrm{E}-04$ & $1.9824 \mathrm{E}-04$ & $2.7159 \mathrm{E}-02$ & $2.1900 \mathrm{E}-04$ & $3.0369 \mathrm{E}-02$ & 0.9052 \\
\hline sm147 & $3.4525 \mathrm{E}+01$ & $5.2892 \mathrm{E}+01$ & $8.7418 \mathrm{E}+01$ & $1.1976 \mathrm{E}+04$ & $9.9655 \mathrm{E}+01$ & $1.3819 \mathrm{E}+04$ & 0.8772 \\
\hline
\end{tabular}


Table 8 (continued)

\begin{tabular}{|c|c|c|c|c|c|c|c|}
\hline \multirow{4}{*}{ Nuclide } & \multicolumn{4}{|c|}{ MOX core } & \multicolumn{2}{|c|}{ LEU core } & \multirow{4}{*}{ Ratios } \\
\hline & \multicolumn{3}{|c|}{ Core-weighted-average components } & \multirow[b]{2}{*}{ Total mass ${ }^{a}$} & \multirow{2}{*}{$\begin{array}{c}\text { Core-weighted } \\
\text { average of LEU } \\
\text { assemblies } \\
\end{array}$} & \multirow[b]{2}{*}{ Total mass $^{a}$} & \\
\hline & $\begin{array}{c}\text { MOX } \\
\text { assemblies }\end{array}$ & $\begin{array}{c}\text { LEU } \\
\text { assemblies }\end{array}$ & $\begin{array}{c}\text { MOX+LEU } \\
\text { assemblies }\end{array}$ & & & & \\
\hline & (g/MTHM) & (g/MTHM) & (g/MTHМ) & (g) & (g/MTHM) & (g) & \\
\hline sm148 & $5.1403 \mathrm{E}+01$ & $7.5439 \mathrm{E}+01$ & $1.2684 \mathrm{E}+02$ & $1.7377 \mathrm{E}+04$ & $1.4554 \mathrm{E}+02$ & $2.0181 \mathrm{E}+04$ & 0.8716 \\
\hline sm149 & $1.2403 \mathrm{E}+00$ & $1.2190 \mathrm{E}+00$ & $2.4593 \mathrm{E}+00$ & $3.3693 \mathrm{E}+02$ & $2.1511 \mathrm{E}+00$ & $2.9830 \mathrm{E}+02$ & 1.1433 \\
\hline sm150 & $1.2462 \mathrm{E}+02$ & $1.6601 \mathrm{E}+02$ & $2.9063 \mathrm{E}+02$ & $3.9816 \mathrm{E}+04$ & $3.1161 \mathrm{E}+02$ & $4.3211 \mathrm{E}+04$ & 0.9327 \\
\hline sm151 & $6.9904 \mathrm{E}+00$ & $6.5826 \mathrm{E}+00$ & $1.3573 \mathrm{E}+01$ & $1.8595 \mathrm{E}+03$ & $1.1825 \mathrm{E}+01$ & $1.6398 \mathrm{E}+03$ & 1.1478 \\
\hline sm152 & $5.7794 \mathrm{E}+01$ & $6.3214 \mathrm{E}+01$ & $1.2101 \mathrm{E}+02$ & $1.6578 \mathrm{E}+04$ & $1.1698 \mathrm{E}+02$ & $1.6221 \mathrm{E}+04$ & 1.0345 \\
\hline sm154 & $2.2657 \mathrm{E}+01$ & $1.9829 \mathrm{E}+01$ & $4.2486 \mathrm{E}+01$ & $5.8206 \mathrm{E}+03$ & $3.7643 \mathrm{E}+01$ & $5.2199 \mathrm{E}+03$ & 1.1287 \\
\hline sn130 & $1.5453 \mathrm{E}-04$ & $2.0440 \mathrm{E}-04$ & $3.5893 \mathrm{E}-04$ & 4.9173E-02 & $3.6689 \mathrm{E}-04$ & $5.0876 \mathrm{E}-02$ & 0.9783 \\
\hline sr89 & $8.1448 \mathrm{E}+00$ & $1.4559 \mathrm{E}+01$ & $2.2704 \mathrm{E}+01$ & $3.1104 \mathrm{E}+03$ & $2.5787 \mathrm{E}+01$ & $3.5759 \mathrm{E}+03$ & 0.8804 \\
\hline sr90 & $1.5161 \mathrm{E}+02$ & $3.3491 \mathrm{E}+02$ & $4.8652 \mathrm{E}+02$ & $6.6653 \mathrm{E}+04$ & $6.2138 \mathrm{E}+02$ & $8.6167 \mathrm{E}+04$ & 0.7830 \\
\hline sr91 & $8.8731 \mathrm{E}-02$ & $1.4877 \mathrm{E}-01$ & $2.3750 \mathrm{E}-01$ & $3.2537 \mathrm{E}+01$ & $2.6383 \mathrm{E}-01$ & $3.6585 \mathrm{E}+01$ & 0.9002 \\
\hline sr92 & $2.8004 \mathrm{E}-02$ & 4.4634E-02 & $7.2638 \mathrm{E}-02$ & $9.9514 \mathrm{E}+00$ & 7.9322E-02 & $1.1000 \mathrm{E}+01$ & 0.9157 \\
\hline sr93 & $1.5326 \mathrm{E}-03$ & $2.3239 \mathrm{E}-03$ & $3.8565 \mathrm{E}-03$ & 5.2834E-01 & $4.1397 \mathrm{E}-03$ & $5.7405 \mathrm{E}-01$ & 0.9316 \\
\hline sr94 & $2.6147 \mathrm{E}-04$ & $3.9117 \mathrm{E}-04$ & $6.5264 \mathrm{E}-04$ & 8.9411E-02 & $6.9723 \mathrm{E}-04$ & $9.6684 \mathrm{E}-02$ & 0.9360 \\
\hline tc101 & $4.6757 \mathrm{E}-03$ & $5.6775 \mathrm{E}-03$ & $1.0353 \mathrm{E}-02$ & $1.4184 \mathrm{E}+00$ & $1.0239 \mathrm{E}-02$ & $1.4199 \mathrm{E}+00$ & 1.0111 \\
\hline tc99 & $3.4380 \mathrm{E}+02$ & $4.7711 \mathrm{E}+02$ & $8.2091 \mathrm{E}+02$ & $1.1246 \mathrm{E}+05$ & $8.8889 \mathrm{E}+02$ & $1.2326 \mathrm{E}+05$ & 0.9235 \\
\hline tc99m & $1.0672 \mathrm{E}-01$ & $1.3546 \mathrm{E}-01$ & $2.4218 \mathrm{E}-01$ & $3.3179 \mathrm{E}+01$ & $2.4360 \mathrm{E}-01$ & $3.3780 \mathrm{E}+01$ & 0.9942 \\
\hline te $125 \mathrm{~m}$ & $6.2694 \mathrm{E}-02$ & $5.7838 \mathrm{E}-02$ & $1.2053 \mathrm{E}-01$ & $1.6513 \mathrm{E}+01$ & $1.0807 \mathrm{E}-01$ & $1.4987 \mathrm{E}+01$ & 1.1153 \\
\hline te127 & $1.4559 \mathrm{E}-02$ & $1.4733 \mathrm{E}-02$ & $2.9291 \mathrm{E}-02$ & $4.0129 \mathrm{E}+00$ & $2.6793 \mathrm{E}-02$ & $3.7154 \mathrm{E}+00$ & 1.0932 \\
\hline te $127 \mathrm{~m}$ & $1.2261 \mathrm{E}-01$ & $1.0901 \mathrm{E}-01$ & $2.3163 \mathrm{E}-01$ & $3.1733 \mathrm{E}+01$ & $1.9585 \mathrm{E}$ & $2.7158 \mathrm{E}+01$ & 1.1827 \\
\hline te129 & $5.4782 \mathrm{E}-03$ & $5.7818 \mathrm{E}-03$ & $1.1260 \mathrm{E}-02$ & $1.5426 \mathrm{E}+00$ & $1.0507 \mathrm{E}-02$ & $1.4570 \mathrm{E}+00$ & 1.0716 \\
\hline te $129 \mathrm{~m}$ & $1.7969 \mathrm{E}-03$ & $1.6971 \mathrm{E}-03$ & 3.4940E-03 & & & & 1.1135 \\
\hline te131 & $5.4951 \mathrm{E}-03$ & $6.7128 \mathrm{E}-03$ & $1.2208 \mathrm{E}-02$ & $1.6725 \mathrm{E}+00$ & $1.2097 \mathrm{E}-02$ & $1.6775 \mathrm{E}+00$ & 1.0092 \\
\hline te $131 \mathrm{~m}$ & $7.2840 \mathrm{E}-02$ & $7.3480 \mathrm{E}-02$ & $1.4632 \mathrm{E}-01$ & $2.0046 \mathrm{E}+01$ & $1.3375 \mathrm{E}-01$ & $1.8547 \mathrm{E}+01$ & 1.0940 \\
\hline te 132 & $1.6312 \mathrm{E}+00$ & $1.9907 \mathrm{E}+00$ & $3.6219 \mathrm{E}+00$ & $4.9620 \mathrm{E}+02$ & $3.5875 \mathrm{E}+00$ & $4.9748 \mathrm{E}+02$ & 1.0096 \\
\hline te133 & $3.4882 \mathrm{E}-03$ & $4.5369 \mathrm{E}-03$ & $8.0251 \mathrm{E}-03$ & $1.0994 \mathrm{E}+00$ & $8.1496 \mathrm{E}-03$ & $1.1301 \mathrm{E}+00$ & 0.9847 \\
\hline te $133 \mathrm{~m}$ & $1.1405 \mathrm{E}-02$ & $1.4634 \mathrm{E}-02$ & $2.6039 \mathrm{E}-02$ & $3.5674 \mathrm{E}+00$ & $2.6286 \mathrm{E}-02$ & $3.6450 \mathrm{E}+00$ & 0.9906 \\
\hline te134 & $1.7267 \mathrm{E}-02$ & $2.3715 \mathrm{E}-02$ & 4.0982E-02 & $5.6145 \mathrm{E}+00$ & $4.2472 \mathrm{E}-02$ & $5.8896 \mathrm{E}+00$ & 0.9649 \\
\hline $\mathrm{u} 234$ & $3.7476 \mathrm{E}+01$ & $1.3726 \mathrm{E}+02$ & $1.7474 \mathrm{E}+02$ & $2.3940 \mathrm{E}+04$ & $2.4126 \mathrm{E}+02$ & $3.3455 \mathrm{E}+04$ & 0.7243 \\
\hline $\mathrm{u} 235$ & $3.2192 \mathrm{E}+03$ & $8.5584 \mathrm{E}+03$ & $1.1778 \mathrm{E}+04$ & $1.6135 \mathrm{E}+06$ & $1.4540 \mathrm{E}+04$ & $2.0163 \mathrm{E}+06$ & 0.8100 \\
\hline $\mathrm{u} 236$ & $6.8441 \mathrm{E}+02$ & $2.8704 \mathrm{E}+03$ & $3.5548 \mathrm{E}+03$ & $4.8700 \mathrm{E}+05$ & $5.2598 \mathrm{E}+03$ & $7.2937 \mathrm{E}+05$ & 0.6758 \\
\hline u237 & $1.4114 \mathrm{E}+00$ & $3.9391 \mathrm{E}+00$ & $5.3505 \mathrm{E}+00$ & $7.3302 \mathrm{E}+02$ & $7.2892 \mathrm{E}+00$ & $1.0108 \mathrm{E}+03$ & 0.7340 \\
\hline u238 & $4.1715 \mathrm{E}+05$ & $5.1769 \mathrm{E}+05$ & $9.3484 \mathrm{E}+05$ & $1.2807 \mathrm{E}+08$ & $9.3199 \mathrm{E}+05$ & $1.2924 \mathrm{E}+08$ & 1.0031 \\
\hline u239 & $1.8153 \mathrm{E}-01$ & $2.2799 \mathrm{E}-01$ & $4.0953 \mathrm{E}-01$ & $5.6105 \mathrm{E}+01$ & $4.1871 \mathrm{E}-01$ & $5.8063 \mathrm{E}+01$ & 0.9781 \\
\hline xe131m & $5.6262 \mathrm{E}-02$ & $6.6714 \mathrm{E}-02$ & $1.2298 \mathrm{E}-01$ & $1.6848 \mathrm{E}+01$ & $1.2084 \mathrm{E}-01$ & $1.6756 \mathrm{E}+01$ & 1.0177 \\
\hline xe133 & $3.6495 \mathrm{E}+00$ & $4.5764 \mathrm{E}+00$ & $8.2259 \mathrm{E}+00$ & $1.1270 \mathrm{E}+03$ & $8.2472 \mathrm{E}+00$ & $1.1436 \mathrm{E}+03$ & 0.9974 \\
\hline xe133m & $2.1242 \mathrm{E}-02$ & $2.4825 \mathrm{E}-02$ & $4.6067 \mathrm{E}-02$ & $6.3112 \mathrm{E}+00$ & $4.5179 \mathrm{E}-02$ & $6.2650 \mathrm{E}+00$ & 1.0196 \\
\hline xe135 & $9.9267 \mathrm{E}-02$ & $1.0289 \mathrm{E}-01$ & $2.0216 \mathrm{E}-01$ & $2.7696 \mathrm{E}+01$ & $1.8139 \mathrm{E}-01$ & $2.5153 \mathrm{E}+01$ & 1.1145 \\
\hline xe135m & $1.2870 \mathrm{E}-03$ & $1.4602 \mathrm{E}-03$ & $2.7472 \mathrm{E}-03$ & \begin{tabular}{|c|}
$3.7637 \mathrm{E}-01$ \\
\end{tabular} & $2.6402 \mathrm{E}-03$ & $3.6612 \mathrm{E}-01$ & 1.0405 \\
\hline xe137 & $1.7504 \mathrm{E}-03$ & $2.2190 \mathrm{E}-03$ & 3.9694E-03 & 5.4380E-01 & $3.9905 \mathrm{E}-03$ & $5.5337 \mathrm{E}-01$ & 0.9947 \\
\hline xe138 & $5.9330 \mathrm{E}-03$ & $7.8416 \mathrm{E}-03$ & $1.3775 \mathrm{E}-02$ & $1.8871 \mathrm{E}+00$ & $1.4073 \mathrm{E}-02$ & $1.9515 \mathrm{E}+00$ & 0.9788 \\
\hline xe139 & $1.9658 \mathrm{E}-04$ & $2.7446 \mathrm{E}-04$ & 4.7103E-04 & 6.4531E-02 & 4.9129E-04 & $6.8127 \mathrm{E}-02$ & 0.9588 \\
\hline xe140 & $4.3420 \mathrm{E}-05$ & $6.4439 \mathrm{E}-05$ & $1.0786 \mathrm{E}-04$ & $1.4777 \mathrm{E}-02$ & $1.1510 \mathrm{E}-04$ & $1.5962 \mathrm{E}-02$ & 0.9371 \\
\hline y90 & $3.9511 \mathrm{E}-02$ & $8.7707 \mathrm{E}-02$ & $1.2722 \mathrm{E}-01$ & $1.7429 \mathrm{E}+01$ & $1.6291 \mathrm{E}-01$ & $2.2591 \mathrm{E}+01$ & 0.7809 \\
\hline y91 & $1.3158 \mathrm{E}+01$ & $2.2292 \mathrm{E}+01$ & $3.5449 \mathrm{E}+01$ & $4.8565 \mathrm{E}+03$ & $3.9577 \mathrm{E}+01$ & $5.4881 \mathrm{E}+03$ & 0.8957 \\
\hline $\mathrm{y} 91 \mathrm{~m}$ & $4.3713 \mathrm{E}-03$ & $7.3277 \mathrm{E}-03$ & $1.1699 \mathrm{E}-02$ & $1.6028 \mathrm{E}+00$ & $1.2994 \mathrm{E}-02$ & $1.8019 \mathrm{E}+00$ & 0.9003 \\
\hline
\end{tabular}


Table 8 (continued)

\begin{tabular}{|c|c|c|c|c|c|c|c|}
\hline \multirow{4}{*}{ Nuclide } & \multicolumn{4}{|c|}{ MOX core } & \multicolumn{2}{|c|}{ LEU core } & \multirow{4}{*}{ Ratios } \\
\hline & \multicolumn{3}{|c|}{ Core-weighted-average components } & \multirow[b]{2}{*}{ Total mass ${ }^{a}$} & \multirow{2}{*}{$\begin{array}{l}\text { Core-weighted } \\
\text { average of LEU } \\
\text { assemblies }\end{array}$} & \multirow[b]{2}{*}{ Total mass ${ }^{a}$} & \\
\hline & $\begin{array}{c}\text { MOX } \\
\text { assemblies }\end{array}$ & $\begin{array}{c}\text { LEU } \\
\text { assemblies }\end{array}$ & $\begin{array}{c}\text { MOX+LEU } \\
\text { assemblies }\end{array}$ & & & & \\
\hline & (g/МТНМ) & (g/МTHМ) & (g/МТНМ) & (g) & (g/МТНМ) & (g) & \\
\hline y92 & $3.7689 \mathrm{E}-02$ & $6.0075 \mathrm{E}-02$ & $9.7763 \mathrm{E}-02$ & $1.3394 \mathrm{E}+01$ & $1.0678 \mathrm{E}-01$ & $1.4808 \mathrm{E}+01$ & 0.9155 \\
\hline y93 & $1.2995 \mathrm{E}-01$ & 1.9589E-01 & $3.2585 \mathrm{E}-01$ & $4.4641 \mathrm{E}+01$ & $3.4903 \mathrm{E}-01$ & $4.8400 \mathrm{E}+01$ & 0.9336 \\
\hline y94 & $4.3668 \mathrm{E}-03$ & $6.3801 \mathrm{E}-03$ & $1.0747 \mathrm{E}-02$ & $1.4723 \mathrm{E}+00$ & $1.1385 \mathrm{E}-02$ & $1.5788 \mathrm{E}+00$ & 0.9439 \\
\hline y95 & $2.6141 \mathrm{E}-03$ & $3.6862 \mathrm{E}-03$ & $6.3003 \mathrm{E}-03$ & $8.6314 \mathrm{E}-01$ & $6.5889 \mathrm{E}-03$ & $9.1368 \mathrm{E}-01$ & 0.9562 \\
\hline y96 & $1.3131 \mathrm{E}-05$ & $1.9392 \mathrm{E}-05$ & $3.2523 \mathrm{E}-05$ & $4.4557 \mathrm{E}-03$ & $3.4621 \mathrm{E}-05$ & 4.8009E-03 & 0.9394 \\
\hline zr95 & $2.4245 \mathrm{E}+01$ & $3.4297 \mathrm{E}+01$ & $5.8542 \mathrm{E}+01$ & $8.0203 \mathrm{E}+03$ & $6.1353 \mathrm{E}+01$ & $8.5078 \mathrm{E}+03$ & 0.9542 \\
\hline zr97 & $2.9000 \mathrm{E}-01$ & $3.8380 \mathrm{E}-01$ & $6.7380 \mathrm{E}-01$ & $9.2310 \mathrm{E}+01$ & $6.8861 \mathrm{E}-01$ & $9.5489 \mathrm{E}+01$ & 0.9759 \\
\hline
\end{tabular}

${ }^{a}$ Core totals based on 137.00 MTHM in MOX core, 138.67 MTHM in LEU core.

Table 9 compares the BFN MOX core/LEU core nuclide ratios for the current study to those reported in Reference [3]. The percentage difference is calculated as

$$
100 *\left(1-\mathrm{R}_{\mathrm{i} \text {-current }} / \mathrm{R}_{\mathrm{i} \text {-EIS-0283 }}\right) \text {. }
$$

Table 9. Comparison of BFN nuclide concentration ratios to Reference [3]

\begin{tabular}{|c|c|c|c|c|}
\hline \multirow{2}{*}{ Nuclide } & \multirow{2}{*}{$\begin{array}{c}\text { Current study } \\
\text { MOX/LEU ratios }\end{array}$} & \multicolumn{2}{|c|}{ EIS-0283 [3] } & \multirow{2}{*}{$\begin{array}{l}\text { Percentage } \\
\text { difference }\end{array}$} \\
\hline & & Nuclide & MOX/LEU ratios & \\
\hline $\operatorname{am} 241$ & 1.9204 & Americium-241 & 2.06 & 6.8 \\
\hline $\mathrm{am} 242$ & 1.6053 & & & \\
\hline $\mathrm{am} 242 \mathrm{~m}$ & 1.9807 & & & \\
\hline $\operatorname{am} 243$ & 1.2745 & & & \\
\hline am244 & 1.2100 & & & \\
\hline am245 & 1.0795 & & & \\
\hline ba137m & 0.9298 & & & \\
\hline ba139 & 0.9831 & Barium-139 & 0.97 & -1.4 \\
\hline ba140 & 0.9806 & Barium-140 & 0.98 & -0.1 \\
\hline ba141 & 0.9797 & Praeseodymium-222 & & \\
\hline ba142 & 0.9708 & & & \\
\hline br83 & 0.9218 & & & \\
\hline br84 & 0.9081 & & & \\
\hline br84m & 1.0783 & & & \\
\hline br85 & 0.8929 & & & \\
\hline br87 & 0.8841 & & & \\
\hline $\operatorname{cd} 112$ & 1.2495 & & & \\
\hline $\operatorname{cd} 114$ & 1.1721 & & & \\
\hline ce140 & 0.8891 & & & \\
\hline ce141 & 0.9802 & Cerium-141 & 0.98 & 0.0 \\
\hline ce142 & 0.8873 & & & \\
\hline ce143 & 0.9624 & Cerium-143 & 0.95 & -1.3 \\
\hline ce144 & 0.9291 & Cerium-144 & 0.91 & -2.1 \\
\hline ce145 & 0.9674 & & & \\
\hline $\mathrm{cm} 242$ & 1.5453 & Curium-242 & 1.43 & -8.1 \\
\hline $\mathrm{cm} 243$ & 1.5494 & & & \\
\hline $\mathrm{cm} 244$ & 1.2113 & Curium-244 & 0.94 & -28.9 \\
\hline
\end{tabular}


Table 9 (continued)

\begin{tabular}{|c|c|c|c|c|}
\hline \multirow{2}{*}{ Nuclide } & \multirow{2}{*}{$\begin{array}{c}\text { Current study } \\
\text { MOX/LEU ratios }\end{array}$} & \multicolumn{2}{|c|}{ EIS-0283 [3] } & \multirow{2}{*}{$\begin{array}{l}\text { Percentage } \\
\text { difference }\end{array}$} \\
\hline & & Nuclide & MOX/LEU ratios & \\
\hline $\mathrm{cm} 245$ & 1.3669 & & & \\
\hline $\mathrm{cm} 246$ & 1.1837 & & & \\
\hline $\operatorname{co5} 5$ & 1.2745 & Cobalt-58 & 0.86 & -48.2 \\
\hline $\operatorname{co6} 60$ & 0.8972 & Cobalt-60 & 0.72 & -24.6 \\
\hline cs133 & 0.9320 & & & \\
\hline $\operatorname{cs} 134$ & 0.8878 & Cesium-134 & 0.85 & -4.4 \\
\hline $\operatorname{cs} 135$ & 1.0301 & & & \\
\hline $\operatorname{cs} 136$ & 1.0131 & Cesium-136 & 1.09 & 7.1 \\
\hline cs137 & 0.9289 & Cesium-137 & 0.91 & -2.1 \\
\hline $\operatorname{cs} 138$ & 0.9839 & & & \\
\hline cs139 & 0.9812 & & & \\
\hline cs 140 & 0.9653 & & & \\
\hline cs141 & 0.9790 & & & \\
\hline eu151 & 1.3755 & & & \\
\hline eu153 & 1.0187 & & & \\
\hline eu154 & 1.0571 & & & \\
\hline eu155 & 1.0347 & & & \\
\hline $\operatorname{gd} 154$ & 0.8997 & & & \\
\hline $\operatorname{gd} 155$ & 0.4446 & & & \\
\hline $\operatorname{gd} 156$ & 0.8908 & & & \\
\hline $\operatorname{gd} 157$ & 1.0896 & & & \\
\hline $\operatorname{gd} 158$ & 0.8908 & & & \\
\hline i129 & 1.0716 & & & \\
\hline $\mathrm{i} 130$ & 0.9769 & & & \\
\hline i131 & 1.0195 & Iodine-131 & 1.03 & 1.0 \\
\hline i132 & 1.0130 & Iodine-132 & 1.02 & 0.7 \\
\hline i133 & 0.9976 & Iodine-133 & 1 & 0.2 \\
\hline $\mathrm{i} 134$ & 0.9901 & Iodine-134 & 0.98 & -1.0 \\
\hline $\mathrm{i} 135$ & 1.0004 & Iodine-135 & 1 & 0.0 \\
\hline $\mathrm{i} 136 \mathrm{~m}$ & 1.0298 & & & \\
\hline $\mathrm{kr} 83 \mathrm{~m}$ & 0.9215 & Krypton- $83 \mathrm{~m}$ & 0.89 & -3.5 \\
\hline $\mathrm{kr} 85$ & 0.7967 & Krypton- 85 & 0.78 & -2.1 \\
\hline $\mathrm{kr} 85 \mathrm{~m}$ & 0.8925 & Krypton- $85 \mathrm{~m}$ & 0.86 & -3.8 \\
\hline kr87 & 0.8902 & Krypton-87 & 0.85 & -4.7 \\
\hline kr88 & 0.8848 & Krypton 88 & 0.84 & -5.3 \\
\hline kr89 & 0.8767 & & & \\
\hline $\mathrm{kr90}$ & 0.8646 & & & \\
\hline la139 & 0.8981 & & & \\
\hline la140 & 0.9757 & Lanthanum-140 & 0.97 & -0.6 \\
\hline la141 & 0.9806 & Lanthanum-141 & 0.97 & -1.1 \\
\hline la142 & 0.9743 & Lanthanum-142 & 0.97 & -0.4 \\
\hline la143 & 0.9628 & & & \\
\hline mo100 & 0.9258 & & & \\
\hline mo92 & 3.3289 & & & \\
\hline mo94 & 0.8342 & & & \\
\hline mo95 & 0.8597 & & & \\
\hline mo96 & 0.7859 & & & \\
\hline mo97 & 0.8975 & & & \\
\hline mo98 & 0.9127 & & & \\
\hline
\end{tabular}


Table 9 (continued)

\begin{tabular}{|c|c|c|c|c|}
\hline \multirow{2}{*}{ Nuclide } & \multirow{2}{*}{$\begin{array}{c}\text { Current study } \\
\text { MOX/LEU ratios }\end{array}$} & \multicolumn{2}{|c|}{ EIS-0283 [3] } & \multirow{2}{*}{$\begin{array}{r}\text { Percentage } \\
\text { difference }\end{array}$} \\
\hline & & Nuclide & MOX/LEU ratios & \\
\hline mo99 & 0.9943 & Molybdenum-99 & 0.99 & -0.4 \\
\hline nb95 & 0.9528 & Niobium-95 & 0.94 & -1.4 \\
\hline nb97 & 0.9795 & & & \\
\hline $\mathrm{nb} 97 \mathrm{~m}$ & 1.1254 & & & \\
\hline nd142 & 0.7839 & & & \\
\hline nd143 & 0.9136 & & & \\
\hline nd144 & 0.8090 & & & \\
\hline nd145 & 0.8862 & & & \\
\hline nd146 & 0.8741 & & & \\
\hline nd147 & 0.9862 & Neodymium-147 & 0.98 & -0.6 \\
\hline nd148 & 0.9157 & & & \\
\hline nd150 & 0.9858 & & & \\
\hline np237 & 0.7296 & & & \\
\hline np238 & 0.7003 & & & \\
\hline np239 & 0.9780 & Neptunium-239 & 0.99 & 1.2 \\
\hline np240 & 0.9478 & & & \\
\hline $\mathrm{pd} 105$ & 1.2153 & & & \\
\hline pd107 & 1.3377 & & & \\
\hline pd108 & 1.3696 & & & \\
\hline $\mathrm{pd} 110$ & 1.3493 & & & \\
\hline pm147 & 0.9492 & & & \\
\hline pr143 & 0.9630 & Praseodymium-143 & 0.95 & -1.4 \\
\hline pr144 & 0.9288 & & & \\
\hline pr145 & 0.9675 & & & \\
\hline pu237 & 0.8889 & & & \\
\hline pu238 & 0.8032 & Plutonium-238 & 0.76 & -5.7 \\
\hline pu239 & 1.5093 & Plutonium-239 & 2.06 & 26.7 \\
\hline $\mathrm{pu} 240$ & 1.7873 & Plutonium-240 & 2.2 & 18.8 \\
\hline pu241 & 1.5667 & Plutonium-241 & 1.79 & 12.5 \\
\hline $\mathrm{pu} 242$ & 1.3473 & & & \\
\hline pu243 & 1.2419 & & & \\
\hline pu244 & 1.1923 & & & \\
\hline pu245 & 1.1423 & & & \\
\hline $\mathrm{rb} 86$ & 0.7821 & Rubidium-86 & 0.77 & -1.6 \\
\hline $\mathrm{rb} 88$ & 0.8872 & & & \\
\hline rb89 & 0.8845 & & & \\
\hline rb90 & 0.8693 & & & \\
\hline rb90m & 0.9555 & & & \\
\hline rb91 & 0.8926 & & & \\
\hline rh103m & 1.0806 & & & \\
\hline rh105 & 1.1494 & Rhodium-105 & 1.19 & 3.4 \\
\hline rh105m & 1.1423 & & & \\
\hline rh106 & 1.2375 & & & \\
\hline rh107 & 1.1964 & & & \\
\hline ru101 & 0.9441 & & & \\
\hline ru102 & 0.9722 & & & \\
\hline ru103 & 1.0805 & Ruthenium-103 & 1.11 & 2.7 \\
\hline ru104 & 1.1200 & & & \\
\hline ru105 & 1.1422 & Ruthenium-105 & 1.18 & 3.2 \\
\hline
\end{tabular}


Table 9 (continued)

\begin{tabular}{|c|c|c|c|c|}
\hline \multirow{2}{*}{ Nuclide } & \multirow{2}{*}{$\begin{array}{c}\text { Current study } \\
\text { MOX/LEU ratios }\end{array}$} & \multicolumn{2}{|c|}{ EIS-0283 [3] } & \multirow{2}{*}{$\begin{array}{l}\text { Percentage } \\
\text { difference }\end{array}$} \\
\hline & & Nuclide & MOX/LEU ratios & \\
\hline ru106 & 1.2610 & Ruthenium-106 & 1.28 & 1.5 \\
\hline ru107 & 1.1965 & & & \\
\hline $\mathrm{sb} 125$ & 1.1137 & & & \\
\hline sb127 & 1.0898 & Antimony-127 & 1.15 & 5.2 \\
\hline sb129 & 1.0716 & Antimony-129 & 1.07 & -0.1 \\
\hline sb130 & 1.0205 & & & \\
\hline sb130m & 1.0033 & & & \\
\hline sb133 & 0.9553 & & & \\
\hline se84 & 0.9052 & & & \\
\hline sm147 & 0.8772 & & & \\
\hline $\operatorname{sm} 148$ & 0.8716 & & & \\
\hline sm149 & 1.1433 & & & \\
\hline $\operatorname{sm} 150$ & 0.9327 & & & \\
\hline sm151 & 1.1478 & & & \\
\hline sm152 & 1.0345 & & & \\
\hline $\operatorname{sm} 154$ & 1.1287 & & & \\
\hline sn130 & 0.9783 & & & \\
\hline sr89 & 0.8804 & Strontium-89 & 0.83 & -6.1 \\
\hline sr90 & 0.7830 & Strontium-90 & 0.75 & -4.4 \\
\hline sr91 & 0.9002 & Strontium-91 & 0.86 & -4.7 \\
\hline sr92 & 0.9157 & Strontium-92 & 0.89 & -2.9 \\
\hline sr93 & 0.9316 & & & \\
\hline sr94 & 0.9360 & & & \\
\hline tc101 & 1.0111 & & & \\
\hline tc99 & 0.9235 & & & \\
\hline tc99m & 0.9942 & Technetium-99m & 0.99 & -0.4 \\
\hline te $125 \mathrm{~m}$ & 1.1153 & & & \\
\hline te127 & 1.0932 & Tellurium-127 & 1.16 & 5.8 \\
\hline te $127 \mathrm{~m}$ & 1.1827 & Tellurium- $127 \mathrm{~m}$ & 1.2 & 1.4 \\
\hline te 129 & 1.0716 & Tellurium-129 & 1.08 & 0.8 \\
\hline te $129 \mathrm{~m}$ & 1.1135 & Tellurium-129m & 1.09 & -2.2 \\
\hline te131 & 1.0092 & & & \\
\hline te $131 \mathrm{~m}$ & 1.0940 & Tellurium-131m & 1.11 & 1.4 \\
\hline te 132 & 1.0096 & Tellurium-132 & 1.01 & 0.0 \\
\hline te133 & 0.9847 & & & \\
\hline te $133 \mathrm{~m}$ & 0.9906 & & & \\
\hline te 134 & 0.9649 & & & \\
\hline u234 & 0.7243 & & & \\
\hline u235 & 0.8100 & & & \\
\hline u236 & 0.6758 & & & \\
\hline u237 & 0.7340 & & & \\
\hline $\mathrm{u} 238$ & 1.0031 & & & \\
\hline u239 & 0.9781 & & & \\
\hline xe131m & 1.0177 & Xenon-131m & 1.02 & 0.2 \\
\hline xe133 & 0.9974 & Xenon-133 & 1 & 0.3 \\
\hline xe133m & 1.0196 & Xenon-133m & 1.01 & -1.0 \\
\hline xe135 & 1.1145 & Xenon-135 & 1.28 & 12.9 \\
\hline xe135m & 1.0405 & Xenon-135m & 1.04 & -0.1 \\
\hline xe137 & 0.9947 & & & \\
\hline
\end{tabular}


Table 9 (continued)

\begin{tabular}{|c|c|c|c|c|}
\hline \multirow{2}{*}{ Nuclide } & \multirow{2}{*}{$\begin{array}{c}\text { Current study } \\
\text { MOX/LEU ratios }\end{array}$} & \multicolumn{2}{|c|}{ EIS-0283 [3] } & \multirow{2}{*}{$\begin{array}{l}\text { Percentage } \\
\text { difference }\end{array}$} \\
\hline & & Nuclide & MOX/LEU ratios & \\
\hline xe138 & 0.9788 & Xenon-138 & 0.96 & -2.0 \\
\hline xe139 & 0.9588 & & & \\
\hline xe140 & 0.9371 & & & \\
\hline y90 & 0.7809 & Yttrium-90 & 0.76 & -2.7 \\
\hline y91 & 0.8957 & Yttrium-91 & 0.85 & -5.4 \\
\hline y91m & 0.9003 & & & \\
\hline y92 & 0.9155 & Yttrium-92 & 0.89 & -2.9 \\
\hline y93 & 0.9336 & Yttrium-93 & 0.91 & -2.6 \\
\hline y94 & 0.9439 & & & \\
\hline y95 & 0.9562 & & & \\
\hline y96 & 0.9394 & & & \\
\hline zr95 & 0.9542 & Zirconium-95 & 0.94 & -1.5 \\
\hline zr97 & 0.9759 & Zirconium-97 & 0.98 & 0.4 \\
\hline
\end{tabular}


Figure 13 graphically presents the MOX core/LEU core nuclide ratio data given in Table 9. The large difference that is apparent for Mo-92 is related to the quite different fission yields of this isotope for plutonium isotopes and U-235.
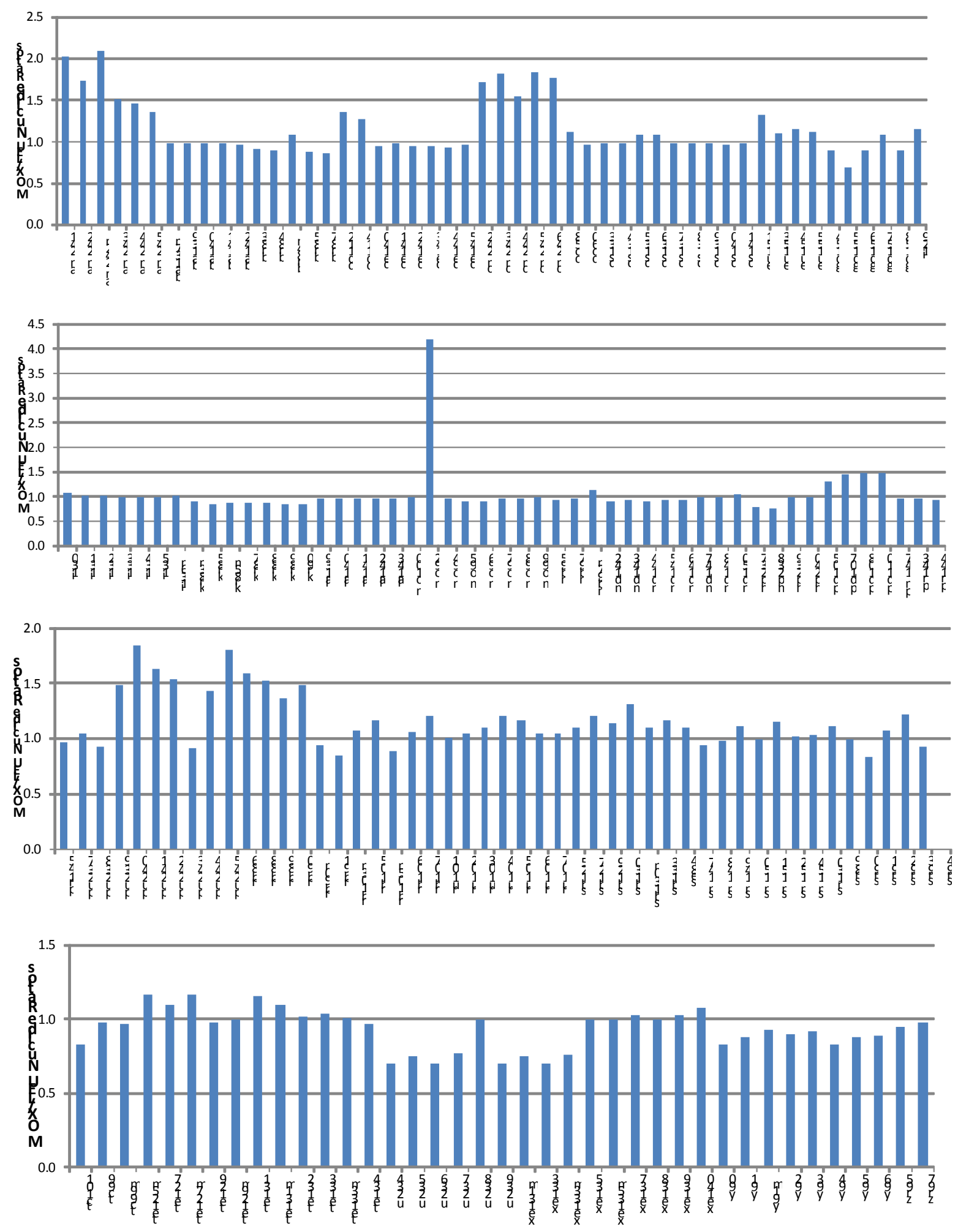

Figure 13. BFN MOX/LEU core-average nuclide ratios. 
Figure 14 presents the percentage differences of the MOX/LEU ratios of the current study to the PWR values of Reference [3] given in Table 9.
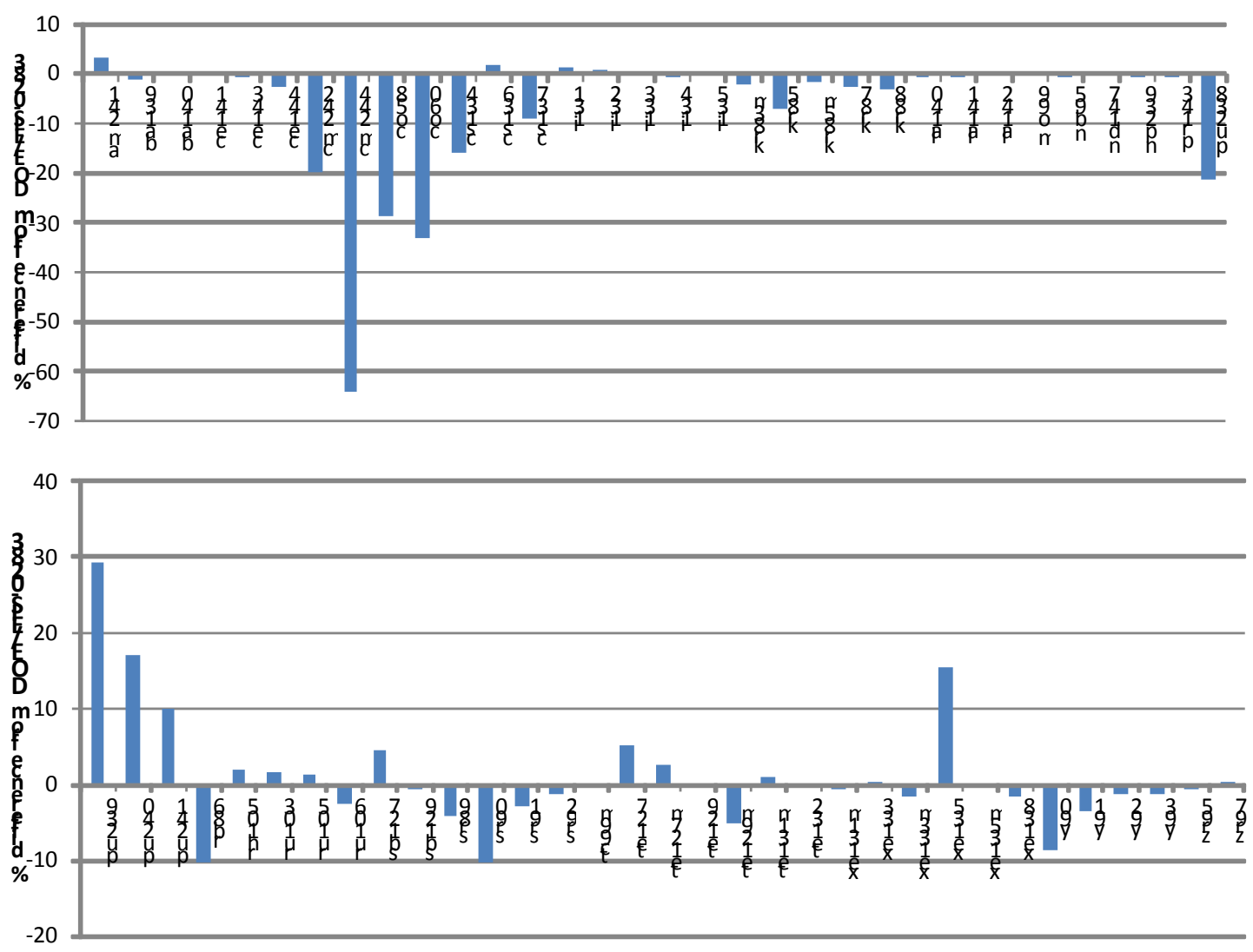

Figure 14. BFN \% differences of MOX/LEU nuclide ratios from DOE/EIS-0283 PWR values.

\subsection{Radioactivity Sources}

\section{SQN}

Table 10 presents a comparison of the contribution of each nuclide to the radioactivity source in the SQN equilibrium MOX and LEU cores. The core-average activity in curies/MTHM as output by SCALE/ORIGEN has been multiplied by the number of metric tonnes of heavy metal in each core to determine the core total activity. There are 87.921 MTHM in the LEU core and 87.302 MTHM in the MOX core. 
Table 10. Comparison of SQN radioactivity sources

\begin{tabular}{|c|c|c|c|c|}
\hline \multirow{2}{*}{ Nuclide } & \multicolumn{2}{|c|}{ MOX core } & \multicolumn{2}{|c|}{ LEU core } \\
\hline & Curies/MTHM & Total curies & Curies/MTHМ & Total curies \\
\hline am241 & $3.20 \mathrm{E}+02$ & $2.79 \mathrm{E}+04$ & $1.53 \mathrm{E}+02$ & $1.35 \mathrm{E}+04$ \\
\hline am242 & $1.63 \mathrm{E}+05$ & $1.42 \mathrm{E}+07$ & $9.39 \mathrm{E}+04$ & $8.25 \mathrm{E}+06$ \\
\hline $\mathrm{am} 242 \mathrm{~m}$ & $1.59 \mathrm{E}+01$ & $1.39 \mathrm{E}+03$ & $7.05 \mathrm{E}+00$ & $6.20 \mathrm{E}+02$ \\
\hline $\operatorname{am} 243$ & $4.13 \mathrm{E}+01$ & $3.60 \mathrm{E}+03$ & $2.59 \mathrm{E}+01$ & $2.28 \mathrm{E}+03$ \\
\hline am244 & $1.62 \mathrm{E}+04$ & $1.42 \mathrm{E}+06$ & $1.05 \mathrm{E}+04$ & $9.20 \mathrm{E}+05$ \\
\hline am245 & $2.64 \mathrm{E}+01$ & $2.30 \mathrm{E}+03$ & $1.78 \mathrm{E}+01$ & $1.57 \mathrm{E}+03$ \\
\hline ba137m & $1.24 \mathrm{E}+05$ & $1.08 \mathrm{E}+07$ & $1.20 \mathrm{E}+05$ & $1.05 \mathrm{E}+07$ \\
\hline ba139 & $1.85 \mathrm{E}+06$ & $1.61 \mathrm{E}+08$ & $1.89 \mathrm{E}+06$ & $1.66 \mathrm{E}+08$ \\
\hline ba140 & $1.77 \mathrm{E}+06$ & $1.54 \mathrm{E}+08$ & $1.82 \mathrm{E}+06$ & $1.60 \mathrm{E}+08$ \\
\hline ba141 & $1.65 \mathrm{E}+06$ & $1.44 \mathrm{E}+08$ & $1.70 \mathrm{E}+06$ & $1.50 \mathrm{E}+08$ \\
\hline ba142 & $1.53 \mathrm{E}+06$ & $1.33 \mathrm{E}+08$ & $1.60 \mathrm{E}+06$ & $1.40 \mathrm{E}+08$ \\
\hline br83 & $1.10 \mathrm{E}+05$ & $9.58 \mathrm{E}+06$ & $1.24 \mathrm{E}+05$ & $1.09 \mathrm{E}+07$ \\
\hline br84 & $1.80 \mathrm{E}+05$ & $1.57 \mathrm{E}+07$ & $2.09 \mathrm{E}+05$ & $1.84 \mathrm{E}+07$ \\
\hline br84m & $1.02 \mathrm{E}+04$ & $8.89 \mathrm{E}+05$ & $9.24 \mathrm{E}+03$ & $8.12 \mathrm{E}+05$ \\
\hline br85 & $2.33 \mathrm{E}+05$ & $2.04 \mathrm{E}+07$ & $2.78 \mathrm{E}+05$ & $2.45 \mathrm{E}+07$ \\
\hline br87 & $3.35 \mathrm{E}+05$ & $2.92 \mathrm{E}+07$ & $4.06 \mathrm{E}+05$ & $3.57 \mathrm{E}+07$ \\
\hline $\operatorname{cd} 112$ & $0.00 \mathrm{E}+00$ & $0.00 \mathrm{E}+00$ & $0.00 \mathrm{E}+00$ & $0.00 \mathrm{E}+00$ \\
\hline $\operatorname{cd} 114$ & $0.00 \mathrm{E}+00$ & $0.00 \mathrm{E}+00$ & $0.00 \mathrm{E}+00$ & $0.00 \mathrm{E}+00$ \\
\hline ce140 & $0.00 \mathrm{E}+00$ & $0.00 \mathrm{E}+00$ & $0.00 \mathrm{E}+00$ & $0.00 \mathrm{E}+00$ \\
\hline ce141 & $1.66 \mathrm{E}+06$ & $1.45 \mathrm{E}+08$ & $1.71 \mathrm{E}+06$ & $1.50 \mathrm{E}+08$ \\
\hline ce142 & $0.00 \mathrm{E}+00$ & $0.00 \mathrm{E}+00$ & $0.00 \mathrm{E}+00$ & $0.00 \mathrm{E}+00$ \\
\hline ce143 & $1.50 \mathrm{E}+06$ & $1.31 \mathrm{E}+08$ & $1.59 \mathrm{E}+06$ & $1.40 \mathrm{E}+08$ \\
\hline ce144 & $1.25 \mathrm{E}+06$ & $1.09 \mathrm{E}+08$ & $1.35 \mathrm{E}+06$ & $1.19 \mathrm{E}+08$ \\
\hline ce145 & $1.04 \mathrm{E}+06$ & $9.04 \mathrm{E}+07$ & $1.09 \mathrm{E}+06$ & $9.55 \mathrm{E}+07$ \\
\hline $\mathrm{cm} 242$ & $9.35 \mathrm{E}+04$ & $8.16 \mathrm{E}+06$ & $5.15 \mathrm{E}+04$ & $4.53 \mathrm{E}+06$ \\
\hline $\mathrm{cm} 243$ & $3.99 \mathrm{E}+01$ & $3.48 \mathrm{E}+03$ & $2.02 \mathrm{E}+01$ & $1.78 \mathrm{E}+03$ \\
\hline $\mathrm{cm} 244$ & $7.38 \mathrm{E}+03$ & $6.44 \mathrm{E}+05$ & $4.23 \mathrm{E}+03$ & $3.72 \mathrm{E}+05$ \\
\hline $\mathrm{cm} 245$ & $1.32 \mathrm{E}+00$ & $1.15 \mathrm{E}+02$ & $6.20 \mathrm{E}-01$ & $5.45 \mathrm{E}+01$ \\
\hline $\mathrm{cm} 246$ & $2.80 \mathrm{E}-01$ & $2.45 \mathrm{E}+01$ & $1.18 \mathrm{E}-01$ & $1.04 \mathrm{E}+01$ \\
\hline $\operatorname{co5} 58$ & $2.93 \mathrm{E}-13$ & $2.56 \mathrm{E}-11$ & $2.21 \mathrm{E}-13$ & $1.95 \mathrm{E}-11$ \\
\hline $\operatorname{co60}$ & $1.34 \mathrm{E}-11$ & $1.17 \mathrm{E}-09$ & $1.36 \mathrm{E}-11$ & 1.19E-09 \\
\hline cs133 & $0.00 \mathrm{E}+00$ & $0.00 \mathrm{E}+00$ & $0.00 \mathrm{E}+00$ & $0.00 \mathrm{E}+00$ \\
\hline cs 134 & $2.27 \mathrm{E}+05$ & $1.98 \mathrm{E}+07$ & $2.11 \mathrm{E}+05$ & $1.86 \mathrm{E}+07$ \\
\hline cs 135 & $6.82 \mathrm{E}-01$ & $5.95 \mathrm{E}+01$ & $5.69 \mathrm{E}-01$ & $5.01 \mathrm{E}+01$ \\
\hline $\operatorname{cs} 136$ & $6.22 \mathrm{E}+04$ & $5.43 \mathrm{E}+06$ & $5.23 \mathrm{E}+04$ & $4.60 \mathrm{E}+06$ \\
\hline $\operatorname{cs} 137$ & $1.30 \mathrm{E}+05$ & $1.14 \mathrm{E}+07$ & $1.26 \mathrm{E}+05$ & $1.11 \mathrm{E}+07$ \\
\hline cs138 & $1.94 \mathrm{E}+06$ & $1.69 \mathrm{E}+08$ & $1.99 \mathrm{E}+06$ & $1.75 \mathrm{E}+08$ \\
\hline cs139 & $1.80 \mathrm{E}+06$ & $1.57 \mathrm{E}+08$ & $1.85 \mathrm{E}+06$ & $1.63 \mathrm{E}+08$ \\
\hline cs 140 & $1.47 \mathrm{E}+06$ & $1.29 \mathrm{E}+08$ & $1.55 \mathrm{E}+06$ & $1.36 \mathrm{E}+08$ \\
\hline cs141 & $1.19 \mathrm{E}+06$ & $1.04 \mathrm{E}+08$ & $1.23 \mathrm{E}+06$ & $1.08 \mathrm{E}+08$ \\
\hline eu151 & $0.00 \mathrm{E}+00$ & $0.00 \mathrm{E}+00$ & $0.00 \mathrm{E}+00$ & $0.00 \mathrm{E}+00$ \\
\hline eu153 & $0.00 \mathrm{E}+00$ & $0.00 \mathrm{E}+00$ & $0.00 \mathrm{E}+00$ & $0.00 \mathrm{E}+00$ \\
\hline eu154 & $1.05 \mathrm{E}+04$ & $9.18 \mathrm{E}+05$ & $8.41 \mathrm{E}+03$ & $7.39 \mathrm{E}+05$ \\
\hline eu155 & $5.55 \mathrm{E}+03$ & $4.85 \mathrm{E}+05$ & $4.64 \mathrm{E}+03$ & $4.08 \mathrm{E}+05$ \\
\hline $\operatorname{gd} 154$ & $0.00 \mathrm{E}+00$ & $0.00 \mathrm{E}+00$ & $0.00 \mathrm{E}+00$ & $0.00 \mathrm{E}+00$ \\
\hline $\operatorname{gd} 155$ & $0.00 \mathrm{E}+00$ & $0.00 \mathrm{E}+00$ & $0.00 \mathrm{E}+00$ & $0.00 \mathrm{E}+00$ \\
\hline $\operatorname{gd} 156$ & $0.00 \mathrm{E}+00$ & $0.00 \mathrm{E}+00$ & $0.00 \mathrm{E}+00$ & $0.00 \mathrm{E}+00$ \\
\hline $\operatorname{gd} 157$ & $0.00 \mathrm{E}+00$ & $0.00 \mathrm{E}+00$ & $0.00 \mathrm{E}+00$ & $0.00 \mathrm{E}+00$ \\
\hline $\operatorname{gd} 158$ & $0.00 \mathrm{E}+00$ & $0.00 \mathrm{E}+00$ & $0.00 \mathrm{E}+00$ & $0.00 \mathrm{E}+00$ \\
\hline
\end{tabular}


Table 10 (continued

\begin{tabular}{|c|c|c|c|c|}
\hline \multirow{2}{*}{ Nuclide } & \multicolumn{2}{|c|}{ MOX core } & \multicolumn{2}{|c|}{ LEU core } \\
\hline & Curies/MTHM & Total curies & Curies/MTHM & Total curies \\
\hline i129 & $3.85 \mathrm{E}-02$ & $3.36 \mathrm{E}+00$ & $3.19 \mathrm{E}-02$ & $2.80 \mathrm{E}+00$ \\
\hline i130 & $2.30 \mathrm{E}+04$ & $2.00 \mathrm{E}+06$ & $2.04 \mathrm{E}+04$ & $1.79 \mathrm{E}+06$ \\
\hline i131 & $1.08 \mathrm{E}+06$ & $9.43 \mathrm{E}+07$ & $1.05 \mathrm{E}+06$ & $9.26 \mathrm{E}+07$ \\
\hline i132 & $1.57 \mathrm{E}+06$ & $1.37 \mathrm{E}+08$ & $1.54 \mathrm{E}+06$ & $1.35 \mathrm{E}+08$ \\
\hline i133 & $2.14 \mathrm{E}+06$ & $1.87 \mathrm{E}+08$ & $2.15 \mathrm{E}+06$ & $1.89 \mathrm{E}+08$ \\
\hline i134 & $2.37 \mathrm{E}+06$ & $2.07 \mathrm{E}+08$ & $2.40 \mathrm{E}+06$ & $2.11 \mathrm{E}+08$ \\
\hline $\mathrm{i} 135$ & $2.05 \mathrm{E}+06$ & $1.79 \mathrm{E}+08$ & $2.05 \mathrm{E}+06$ & $1.80 \mathrm{E}+08$ \\
\hline $\mathrm{i} 136 \mathrm{~m}$ & $4.96 \mathrm{E}+05$ & $4.33 \mathrm{E}+07$ & $4.77 \mathrm{E}+05$ & $4.19 \mathrm{E}+07$ \\
\hline kr83m & $1.09 \mathrm{E}+05$ & $9.54 \mathrm{E}+06$ & $1.24 \mathrm{E}+05$ & $1.09 \mathrm{E}+07$ \\
\hline kr85 & $1.25 \mathrm{E}+04$ & $1.09 \mathrm{E}+06$ & $1.46 \mathrm{E}+04$ & $1.29 \mathrm{E}+06$ \\
\hline kr85m & $2.25 \mathrm{E}+05$ & $1.97 \mathrm{E}+07$ & $2.69 \mathrm{E}+05$ & $2.36 \mathrm{E}+07$ \\
\hline kr87 & $4.30 \mathrm{E}+05$ & $3.75 \mathrm{E}+07$ & $5.15 \mathrm{E}+05$ & $4.53 \mathrm{E}+07$ \\
\hline kr88 & $5.63 \mathrm{E}+05$ & $4.91 \mathrm{E}+07$ & $6.82 \mathrm{E}+05$ & $5.99 \mathrm{E}+07$ \\
\hline kr89 & $6.90 \mathrm{E}+05$ & $6.02 \mathrm{E}+07$ & $8.48 \mathrm{E}+05$ & $7.46 \mathrm{E}+07$ \\
\hline kr90 & $6.95 \mathrm{E}+05$ & $6.07 \mathrm{E}+07$ & $8.77 \mathrm{E}+05$ & $7.71 \mathrm{E}+07$ \\
\hline la139 & $0.00 \mathrm{E}+00$ & $0.00 \mathrm{E}+00$ & $0.00 \mathrm{E}+00$ & $0.00 \mathrm{E}+00$ \\
\hline la140 & $1.92 \mathrm{E}+06$ & $1.68 \mathrm{E}+08$ & $1.98 \mathrm{E}+06$ & $1.74 \mathrm{E}+08$ \\
\hline la141 & $1.67 \mathrm{E}+06$ & $1.45 \mathrm{E}+08$ & $1.72 \mathrm{E}+06$ & $1.51 \mathrm{E}+08$ \\
\hline la142 & $1.59 \mathrm{E}+06$ & $1.38 \mathrm{E}+08$ & $1.65 \mathrm{E}+06$ & $1.45 \mathrm{E}+08$ \\
\hline la143 & $1.49 \mathrm{E}+06$ & $1.30 \mathrm{E}+08$ & $1.58 \mathrm{E}+06$ & $1.39 \mathrm{E}+08$ \\
\hline mo100 & $5.31 \mathrm{E}-13$ & $4.63 \mathrm{E}-11$ & $5.11 \mathrm{E}-13$ & $4.49 \mathrm{E}-11$ \\
\hline mo92 & $0.00 \mathrm{E}+00$ & $0.00 \mathrm{E}+00$ & $0.00 \mathrm{E}+00$ & $0.00 \mathrm{E}+00$ \\
\hline mo94 & $0.00 \mathrm{E}+00$ & $0.00 \mathrm{E}+00$ & $0.00 \mathrm{E}+00$ & $0.00 \mathrm{E}+00$ \\
\hline mo95 & $0.00 \mathrm{E}+00$ & $0.00 \mathrm{E}+00$ & $0.00 \mathrm{E}+00$ & $0.00 \mathrm{E}+00$ \\
\hline mo96 & $0.00 \mathrm{E}+00$ & $0.00 \mathrm{E}+00$ & $0.00 \mathrm{E}+00$ & $0.00 \mathrm{E}+00$ \\
\hline mo97 & $0.00 \mathrm{E}+00$ & $0.00 \mathrm{E}+00$ & $0.00 \mathrm{E}+00$ & $0.00 \mathrm{E}+00$ \\
\hline mo98 & $0.00 \mathrm{E}+00$ & $0.00 \mathrm{E}+00$ & $0.00 \mathrm{E}+00$ & $0.00 \mathrm{E}+00$ \\
\hline mo99 & $1.94 \mathrm{E}+06$ & $1.69 \mathrm{E}+08$ & $1.95 \mathrm{E}+06$ & $1.71 \mathrm{E}+08$ \\
\hline nb95 & $1.62 \mathrm{E}+06$ & $1.41 \mathrm{E}+08$ & $1.74 \mathrm{E}+06$ & $1.53 \mathrm{E}+08$ \\
\hline nb97 & $1.73 \mathrm{E}+06$ & $1.51 \mathrm{E}+08$ & $1.79 \mathrm{E}+06$ & $1.57 \mathrm{E}+08$ \\
\hline nb97m & $2.73 \mathrm{E}+03$ & $2.38 \mathrm{E}+05$ & $2.36 \mathrm{E}+03$ & $2.07 \mathrm{E}+05$ \\
\hline nd142 & $0.00 \mathrm{E}+00$ & $0.00 \mathrm{E}+00$ & $0.00 \mathrm{E}+00$ & $0.00 \mathrm{E}+00$ \\
\hline nd143 & $0.00 \mathrm{E}+00$ & $0.00 \mathrm{E}+00$ & $0.00 \mathrm{E}+00$ & $0.00 \mathrm{E}+00$ \\
\hline nd144 & 1.19E-09 & $1.04 \mathrm{E}-07$ & $1.23 \mathrm{E}-09$ & $1.08 \mathrm{E}-07$ \\
\hline nd145 & $0.00 \mathrm{E}+00$ & $0.00 \mathrm{E}+00$ & $0.00 \mathrm{E}+00$ & $0.00 \mathrm{E}+00$ \\
\hline nd146 & $0.00 \mathrm{E}+00$ & $0.00 \mathrm{E}+00$ & $0.00 \mathrm{E}+00$ & $0.00 \mathrm{E}+00$ \\
\hline nd147 & $6.69 \mathrm{E}+05$ & $5.84 \mathrm{E}+07$ & $6.81 \mathrm{E}+05$ & $5.99 \mathrm{E}+07$ \\
\hline nd148 & $0.00 \mathrm{E}+00$ & $0.00 \mathrm{E}+00$ & $0.00 \mathrm{E}+00$ & $0.00 \mathrm{E}+00$ \\
\hline nd150 & $7.03 \mathrm{E}-14$ & $6.14 \mathrm{E}-12$ & $6.31 \mathrm{E}-14$ & $5.55 \mathrm{E}-12$ \\
\hline np237 & $3.20 \mathrm{E}-01$ & $2.79 \mathrm{E}+01$ & $3.94 \mathrm{E}-01$ & $3.47 \mathrm{E}+01$ \\
\hline np238 & $3.72 \mathrm{E}+05$ & $3.24 \mathrm{E}+07$ & $4.51 \mathrm{E}+05$ & $3.97 \mathrm{E}+07$ \\
\hline np239 & $2.18 \mathrm{E}+07$ & $1.91 \mathrm{E}+09$ & $2.17 \mathrm{E}+07$ & $1.90 \mathrm{E}+09$ \\
\hline np240 & $1.50 \mathrm{E}+04$ & $1.31 \mathrm{E}+06$ & $1.49 \mathrm{E}+04$ & $1.31 \mathrm{E}+06$ \\
\hline pd105 & $0.00 \mathrm{E}+00$ & $0.00 \mathrm{E}+00$ & $0.00 \mathrm{E}+00$ & $0.00 \mathrm{E}+00$ \\
\hline pd107 & $1.89 \mathrm{E}-01$ & $1.65 \mathrm{E}+01$ & $1.25 \mathrm{E}-01$ & $1.10 \mathrm{E}+01$ \\
\hline pd108 & $0.00 \mathrm{E}+00$ & $0.00 \mathrm{E}+00$ & $0.00 \mathrm{E}+00$ & $0.00 \mathrm{E}+00$ \\
\hline pd110 & $0.00 \mathrm{E}+00$ & $0.00 \mathrm{E}+00$ & $0.00 \mathrm{E}+00$ & $0.00 \mathrm{E}+00$ \\
\hline pm147 & $1.90 \mathrm{E}+05$ & $1.66 \mathrm{E}+07$ & $1.98 \mathrm{E}+05$ & $1.74 \mathrm{E}+07$ \\
\hline pr143 & $1.45 \mathrm{E}+06$ & $1.27 \mathrm{E}+08$ & $1.54 \mathrm{E}+06$ & $1.35 \mathrm{E}+08$ \\
\hline
\end{tabular}


Table 10 (continued

\begin{tabular}{|c|c|c|c|c|}
\hline \multirow{2}{*}{ Nuclide } & \multicolumn{2}{|c|}{ MOX core } & \multicolumn{2}{|c|}{ LEU core } \\
\hline & Curies/MTHM & Total curies & Curies/MTHM & Total curies \\
\hline pr144 & $1.26 \mathrm{E}+06$ & $1.10 \mathrm{E}+08$ & $1.36 \mathrm{E}+06$ & $1.20 \mathrm{E}+08$ \\
\hline pr145 & $1.04 \mathrm{E}+06$ & $9.05 \mathrm{E}+07$ & $1.09 \mathrm{E}+06$ & $9.56 \mathrm{E}+07$ \\
\hline pu237 & $8.37 \mathrm{E}+00$ & $7.30 \mathrm{E}+02$ & $7.38 \mathrm{E}+00$ & $6.49 \mathrm{E}+02$ \\
\hline pu238 & $3.54 \mathrm{E}+03$ & $3.09 \mathrm{E}+05$ & $3.57 \mathrm{E}+03$ & $3.14 \mathrm{E}+05$ \\
\hline pu239 & $6.30 \mathrm{E}+02$ & $5.50 \mathrm{E}+04$ & $3.93 \mathrm{E}+02$ & $3.46 \mathrm{E}+04$ \\
\hline pu240 & $1.02 \mathrm{E}+03$ & $8.95 \mathrm{E}+04$ & $5.31 \mathrm{E}+02$ & $4.67 \mathrm{E}+04$ \\
\hline pu241 & $2.63 \mathrm{E}+05$ & $2.30 \mathrm{E}+07$ & $1.54 \mathrm{E}+05$ & $1.35 \mathrm{E}+07$ \\
\hline $\mathrm{pu} 242$ & $3.22 \mathrm{E}+00$ & $2.81 \mathrm{E}+02$ & $2.09 \mathrm{E}+00$ & $1.84 \mathrm{E}+02$ \\
\hline pu243 & $6.16 \mathrm{E}+05$ & $5.38 \mathrm{E}+07$ & $4.27 \mathrm{E}+05$ & $3.75 \mathrm{E}+07$ \\
\hline pu244 & $1.25 \mathrm{E}-06$ & $1.09 \mathrm{E}-04$ & $7.35 \mathrm{E}-07$ & $6.46 \mathrm{E}-05$ \\
\hline pu245 & $6.00 \mathrm{E}+00$ & $5.24 \mathrm{E}+02$ & $3.43 \mathrm{E}+00$ & $3.01 \mathrm{E}+02$ \\
\hline $\mathrm{rb} 86$ & $2.68 \mathrm{E}+03$ & $2.34 \mathrm{E}+05$ & $3.00 \mathrm{E}+03$ & $2.64 \mathrm{E}+05$ \\
\hline $\mathrm{rb} 88$ & $5.76 \mathrm{E}+05$ & $5.03 \mathrm{E}+07$ & $6.95 \mathrm{E}+05$ & $6.11 \mathrm{E}+07$ \\
\hline rb89 & $7.53 \mathrm{E}+05$ & $6.58 \mathrm{E}+07$ & $9.13 \mathrm{E}+05$ & $8.03 \mathrm{E}+07$ \\
\hline rb90 & $7.37 \mathrm{E}+05$ & $6.43 \mathrm{E}+07$ & $9.20 \mathrm{E}+05$ & $8.09 \mathrm{E}+07$ \\
\hline rb90m & $1.67 \mathrm{E}+05$ & $1.46 \mathrm{E}+07$ & $1.79 \mathrm{E}+05$ & $1.57 \mathrm{E}+07$ \\
\hline $\mathrm{rb91}$ & $9.37 \mathrm{E}+05$ & $8.18 \mathrm{E}+07$ & $1.12 \mathrm{E}+06$ & $9.83 \mathrm{E}+07$ \\
\hline rh103m & $1.86 \mathrm{E}+06$ & $1.62 \mathrm{E}+08$ & $1.67 \mathrm{E}+06$ & $1.47 \mathrm{E}+08$ \\
\hline rh105 & $1.33 \mathrm{E}+06$ & $1.16 \mathrm{E}+08$ & $1.11 \mathrm{E}+06$ & $9.77 \mathrm{E}+07$ \\
\hline rh105m & $4.02 \mathrm{E}+05$ & $3.51 \mathrm{E}+07$ & $3.39 \mathrm{E}+05$ & $2.98 \mathrm{E}+07$ \\
\hline rh106 & $8.99 \mathrm{E}+05$ & $7.85 \mathrm{E}+07$ & $6.76 \mathrm{E}+05$ & $5.94 \mathrm{E}+07$ \\
\hline rh107 & $8.41 \mathrm{E}+05$ & $7.35 \mathrm{E}+07$ & $6.73 \mathrm{E}+05$ & $5.92 \mathrm{E}+07$ \\
\hline ru101 & $0.00 \mathrm{E}+00$ & $0.00 \mathrm{E}+00$ & $0.00 \mathrm{E}+00$ & $0.00 \mathrm{E}+00$ \\
\hline ru102 & $0.00 \mathrm{E}+00$ & $0.00 \mathrm{E}+00$ & $0.00 \mathrm{E}+00$ & $0.00 \mathrm{E}+00$ \\
\hline ru103 & $1.88 \mathrm{E}+06$ & $1.64 \mathrm{E}+08$ & $1.69 \mathrm{E}+06$ & $1.49 \mathrm{E}+08$ \\
\hline ru104 & $0.00 \mathrm{E}+00$ & $0.00 \mathrm{E}+00$ & $0.00 \mathrm{E}+00$ & $0.00 \mathrm{E}+00$ \\
\hline ru105 & $1.43 \mathrm{E}+06$ & $1.24 \mathrm{E}+08$ & $1.20 \mathrm{E}+06$ & $1.06 \mathrm{E}+08$ \\
\hline ru106 & $8.11 \mathrm{E}+05$ & $7.08 \mathrm{E}+07$ & $5.94 \mathrm{E}+05$ & $5.22 \mathrm{E}+07$ \\
\hline ru107 & $8.29 \mathrm{E}+05$ & $7.24 \mathrm{E}+07$ & $6.63 \mathrm{E}+05$ & $5.83 \mathrm{E}+07$ \\
\hline sb125 & $1.30 \mathrm{E}+04$ & $1.13 \mathrm{E}+06$ & $1.06 \mathrm{E}+04$ & $9.36 \mathrm{E}+05$ \\
\hline sb127 & $1.15 \mathrm{E}+05$ & $1.00 \mathrm{E}+07$ & $1.02 \mathrm{E}+05$ & $9.00 \mathrm{E}+06$ \\
\hline sb129 & $3.38 \mathrm{E}+05$ & $2.95 \mathrm{E}+07$ & $3.09 \mathrm{E}+05$ & $2.72 \mathrm{E}+07$ \\
\hline sb130 & $3.10 \mathrm{E}+05$ & $2.70 \mathrm{E}+07$ & $3.01 \mathrm{E}+05$ & $2.65 \mathrm{E}+07$ \\
\hline sb130m & $3.16 \mathrm{E}+05$ & $2.76 \mathrm{E}+07$ & $3.15 \mathrm{E}+05$ & $2.77 \mathrm{E}+07$ \\
\hline sb133 & $5.98 \mathrm{E}+05$ & $5.22 \mathrm{E}+07$ & $6.38 \mathrm{E}+05$ & $5.61 \mathrm{E}+07$ \\
\hline se84 & $1.74 \mathrm{E}+05$ & $1.52 \mathrm{E}+07$ & $2.03 \mathrm{E}+05$ & $1.78 \mathrm{E}+07$ \\
\hline sm147 & $1.82 \mathrm{E}-06$ & $1.59 \mathrm{E}-04$ & $1.85 \mathrm{E}-06$ & $1.62 \mathrm{E}-04$ \\
\hline sm148 & $6.08 \mathrm{E}-11$ & $5.30 \mathrm{E}-09$ & $5.65 \mathrm{E}-11$ & 4.97E-09 \\
\hline sm149 & $0.00 \mathrm{E}+00$ & $0.00 \mathrm{E}+00$ & $0.00 \mathrm{E}+00$ & $0.00 \mathrm{E}+00$ \\
\hline sm150 & $0.00 \mathrm{E}+00$ & $0.00 \mathrm{E}+00$ & $0.00 \mathrm{E}+00$ & $0.00 \mathrm{E}+00$ \\
\hline sm151 & $4.81 \mathrm{E}+02$ & $4.20 \mathrm{E}+04$ & $3.86 \mathrm{E}+02$ & $3.39 \mathrm{E}+04$ \\
\hline sm152 & $0.00 \mathrm{E}+00$ & $0.00 \mathrm{E}+00$ & $0.00 \mathrm{E}+00$ & $0.00 \mathrm{E}+00$ \\
\hline sm154 & $0.00 \mathrm{E}+00$ & $0.00 \mathrm{E}+00$ & $0.00 \mathrm{E}+00$ & $0.00 \mathrm{E}+00$ \\
\hline sn130 & $1.85 \mathrm{E}+05$ & $1.61 \mathrm{E}+07$ & $1.90 \mathrm{E}+05$ & $1.67 \mathrm{E}+07$ \\
\hline sr89 & $7.79 \mathrm{E}+05$ & $6.80 \mathrm{E}+07$ & $9.51 \mathrm{E}+05$ & $8.36 \mathrm{E}+07$ \\
\hline sr90 & $7.66 \mathrm{E}+04$ & $6.69 \mathrm{E}+06$ & $9.03 \mathrm{E}+04$ & $7.93 \mathrm{E}+06$ \\
\hline sr91 & $1.02 \mathrm{E}+06$ & $8.93 \mathrm{E}+07$ & $1.20 \mathrm{E}+06$ & $1.06 \mathrm{E}+08$ \\
\hline sr92 & $1.14 \mathrm{E}+06$ & $9.98 \mathrm{E}+07$ & $1.31 \mathrm{E}+06$ & $1.15 \mathrm{E}+08$ \\
\hline sr93 & $1.32 \mathrm{E}+06$ & $1.15 \mathrm{E}+08$ & $1.46 \mathrm{E}+06$ & $1.29 \mathrm{E}+08$ \\
\hline
\end{tabular}


Table 10 (continued)

\begin{tabular}{|c|c|c|c|c|}
\hline \multirow{2}{*}{ Nuclide } & \multicolumn{2}{|c|}{ MOX core } & \multicolumn{2}{|c|}{ LEU core } \\
\hline & Curies/MTHM & Total curies & Curies/MTHM & Total curies \\
\hline sr94 & $1.31 \mathrm{E}+06$ & $1.14 \mathrm{E}+08$ & $1.45 \mathrm{E}+06$ & $1.27 \mathrm{E}+08$ \\
\hline tc101 & $1.85 \mathrm{E}+06$ & $1.61 \mathrm{E}+08$ & $1.82 \mathrm{E}+06$ & $1.60 \mathrm{E}+08$ \\
\hline tc99 & $1.62 \mathrm{E}+01$ & $1.42 \mathrm{E}+03$ & $1.60 \mathrm{E}+01$ & $1.40 \mathrm{E}+03$ \\
\hline tc99m & $1.71 \mathrm{E}+06$ & $1.49 \mathrm{E}+08$ & $1.73 \mathrm{E}+06$ & $1.52 \mathrm{E}+08$ \\
\hline te $125 \mathrm{~m}$ & $2.84 \mathrm{E}+03$ & $2.48 \mathrm{E}+05$ & $2.30 \mathrm{E}+03$ & $2.02 \mathrm{E}+05$ \\
\hline te 127 & $1.12 \mathrm{E}+05$ & $9.77 \mathrm{E}+06$ & $9.98 \mathrm{E}+04$ & $8.77 \mathrm{E}+06$ \\
\hline te $127 \mathrm{~m}$ & $3.05 \mathrm{E}+03$ & $2.66 \mathrm{E}+05$ & $2.48 \mathrm{E}+03$ & $2.18 \mathrm{E}+05$ \\
\hline te129 & $3.39 \mathrm{E}+05$ & $2.96 \mathrm{E}+07$ & $3.09 \mathrm{E}+05$ & $2.72 \mathrm{E}+07$ \\
\hline te $129 \mathrm{~m}$ & $1.65 \mathrm{E}+02$ & $1.44 \mathrm{E}+04$ & $1.38 \mathrm{E}+02$ & $1.22 \mathrm{E}+04$ \\
\hline te131 & $9.53 \mathrm{E}+05$ & $8.32 \mathrm{E}+07$ & $9.42 \mathrm{E}+05$ & $8.28 \mathrm{E}+07$ \\
\hline te $131 \mathrm{~m}$ & $1.70 \mathrm{E}+05$ & $1.49 \mathrm{E}+07$ & $1.52 \mathrm{E}+05$ & $1.33 \mathrm{E}+07$ \\
\hline te132 & $1.52 \mathrm{E}+06$ & $1.33 \mathrm{E}+08$ & $1.50 \mathrm{E}+06$ & $1.32 \mathrm{E}+08$ \\
\hline te 133 & $1.21 \mathrm{E}+06$ & $1.05 \mathrm{E}+08$ & $1.23 \mathrm{E}+06$ & $1.08 \mathrm{E}+08$ \\
\hline te $133 \mathrm{~m}$ & $8.85 \mathrm{E}+05$ & $7.73 \mathrm{E}+07$ & $8.98 \mathrm{E}+05$ & $7.90 \mathrm{E}+07$ \\
\hline te 134 & $1.79 \mathrm{E}+06$ & $1.56 \mathrm{E}+08$ & $1.88 \mathrm{E}+06$ & $1.66 \mathrm{E}+08$ \\
\hline u234 & $8.46 \mathrm{E}-01$ & $7.38 \mathrm{E}+01$ & $1.40 \mathrm{E}+00$ & $1.23 \mathrm{E}+02$ \\
\hline $\mathrm{u} 235$ & $1.72 \mathrm{E}-02$ & $1.50 \mathrm{E}+00$ & $2.95 \mathrm{E}-02$ & $2.59 \mathrm{E}+00$ \\
\hline $\mathrm{u} 236$ & $2.37 \mathrm{E}-01$ & $2.07 \mathrm{E}+01$ & $3.52 \mathrm{E}-01$ & $3.09 \mathrm{E}+01$ \\
\hline $\mathrm{u} 237$ & $7.42 \mathrm{E}+05$ & $6.48 \mathrm{E}+07$ & $9.82 \mathrm{E}+05$ & $8.63 \mathrm{E}+07$ \\
\hline $\mathrm{u} 238$ & $3.12 \mathrm{E}-01$ & $2.72 \mathrm{E}+01$ & $3.12 \mathrm{E}-01$ & $2.74 \mathrm{E}+01$ \\
\hline u239 & $2.19 \mathrm{E}+07$ & $1.91 \mathrm{E}+09$ & $2.17 \mathrm{E}+07$ & $1.91 \mathrm{E}+09$ \\
\hline $\mathrm{xe} 131 \mathrm{~m}$ & $1.43 \mathrm{E}+04$ & $1.25 \mathrm{E}+06$ & $1.38 \mathrm{E}+04$ & $1.22 \mathrm{E}+06$ \\
\hline xe133 & $2.06 \mathrm{E}+06$ & $1.80 \mathrm{E}+08$ & $2.06 \mathrm{E}+06$ & $1.82 \mathrm{E}+08$ \\
\hline xe133m & $2.84 \mathrm{E}+04$ & $2.48 \mathrm{E}+06$ & $2.74 \mathrm{E}+04$ & $2.41 \mathrm{E}+06$ \\
\hline xe135 & $6.90 \mathrm{E}+05$ & $6.03 \mathrm{E}+07$ & $6.02 \mathrm{E}+05$ & $5.30 \mathrm{E}+07$ \\
\hline xe135m & $3.49 \mathrm{E}+05$ & $3.05 \mathrm{E}+07$ & $3.32 \mathrm{E}+05$ & $2.92 \mathrm{E}+07$ \\
\hline xe137 & $1.91 \mathrm{E}+06$ & $1.67 \mathrm{E}+08$ & $1.93 \mathrm{E}+06$ & $1.69 \mathrm{E}+08$ \\
\hline xe138 & $1.76 \mathrm{E}+06$ & $1.53 \mathrm{E}+08$ & $1.81 \mathrm{E}+06$ & $1.59 \mathrm{E}+08$ \\
\hline xe139 & $1.24 \mathrm{E}+06$ & $1.09 \mathrm{E}+08$ & $1.32 \mathrm{E}+06$ & $1.16 \mathrm{E}+08$ \\
\hline xe140 & $8.08 \mathrm{E}+05$ & $7.06 \mathrm{E}+07$ & $8.88 \mathrm{E}+05$ & $7.81 \mathrm{E}+07$ \\
\hline y90 & $7.93 \mathrm{E}+04$ & $6.92 \mathrm{E}+06$ & $9.34 \mathrm{E}+04$ & $8.21 \mathrm{E}+06$ \\
\hline y91 & $1.05 \mathrm{E}+06$ & $9.17 \mathrm{E}+07$ & $1.24 \mathrm{E}+06$ & $1.09 \mathrm{E}+08$ \\
\hline $\mathrm{y} 91 \mathrm{~m}$ & $5.86 \mathrm{E}+05$ & $5.12 \mathrm{E}+07$ & $6.89 \mathrm{E}+05$ & $6.06 \mathrm{E}+07$ \\
\hline y92 & $1.16 \mathrm{E}+06$ & $1.01 \mathrm{E}+08$ & $1.32 \mathrm{E}+06$ & $1.16 \mathrm{E}+08$ \\
\hline y93 & $1.35 \mathrm{E}+06$ & $1.18 \mathrm{E}+08$ & $1.50 \mathrm{E}+06$ & $1.32 \mathrm{E}+08$ \\
\hline y94 & $1.46 \mathrm{E}+06$ & $1.27 \mathrm{E}+08$ & $1.59 \mathrm{E}+06$ & $1.40 \mathrm{E}+08$ \\
\hline y95 & $1.56 \mathrm{E}+06$ & $1.36 \mathrm{E}+08$ & $1.67 \mathrm{E}+06$ & $1.47 \mathrm{E}+08$ \\
\hline y96 & $9.08 \mathrm{E}+05$ & $7.93 \mathrm{E}+07$ & $9.96 \mathrm{E}+05$ & $8.75 \mathrm{E}+07$ \\
\hline zr95 & $1.61 \mathrm{E}+06$ & $1.41 \mathrm{E}+08$ & $1.73 \mathrm{E}+06$ & $1.52 \mathrm{E}+08$ \\
\hline zr97 & $1.72 \mathrm{E}+06$ & $1.50 \mathrm{E}+08$ & $1.77 \mathrm{E}+06$ & $1.56 \mathrm{E}+08$ \\
\hline
\end{tabular}


Figure 15 graphically presents the MOX/LEU curie ratios of the total core data presented in Table 10.
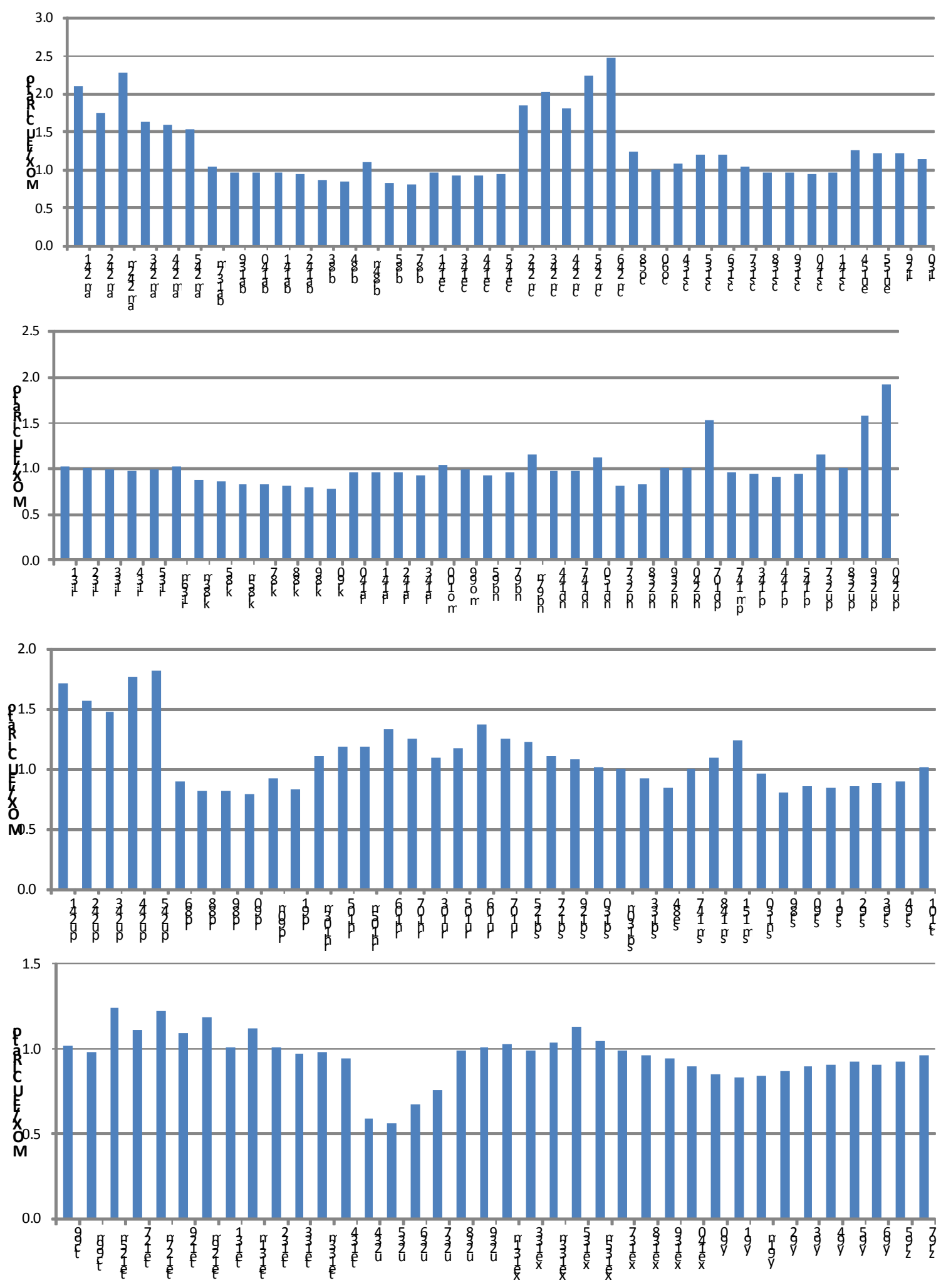

Figure 15. SQN MOX/LEU core-average curie ratios. 


\section{BFN}

Table 11 presents a comparison of the contribution of each nuclide to the radioactivity source in the BFN equilibrium MOX and LEU cores. The activity in curies/MTHM as output by SCALE/ORIGEN has been multiplied by the number of metric tonnes of heavy metal in each core to determine the core total activity. There are 138.67 MTHM in the LEU core and 137.00 MTHM in the MOX core.

Table 11. Comparison of BFN radioactivity sources

\begin{tabular}{|c|c|c|c|c|}
\hline \multirow{2}{*}{ Nuclide } & \multicolumn{2}{|c|}{ MOX core } & \multicolumn{2}{|c|}{ LEU core } \\
\hline & Curies/MTHM & Total curies & Curies/MTHM & Total curies \\
\hline am241 & $2.54 \mathrm{E}+02$ & $3.48 \mathrm{E}+04$ & $1.39 \mathrm{E}+02$ & $1.92 \mathrm{E}+04$ \\
\hline am242 & $1.08 \mathrm{E}+05$ & $1.48 \mathrm{E}+07$ & $7.01 \mathrm{E}+04$ & $9.72 \mathrm{E}+06$ \\
\hline am $242 m$ & $1.03 \mathrm{E}+01$ & $1.41 \mathrm{E}+03$ & $5.46 \mathrm{E}+00$ & $7.57 \mathrm{E}+02$ \\
\hline am243 & $2.58 \mathrm{E}+01$ & $3.53 \mathrm{E}+03$ & $2.03 \mathrm{E}+01$ & $2.81 \mathrm{E}+03$ \\
\hline $\operatorname{am} 244$ & $7.03 \mathrm{E}+03$ & $9.63 \mathrm{E}+05$ & $5.82 \mathrm{E}+03$ & $8.06 \mathrm{E}+05$ \\
\hline $\operatorname{am} 245$ & $1.04 \mathrm{E}+01$ & $1.43 \mathrm{E}+03$ & $9.68 \mathrm{E}+00$ & $1.34 \mathrm{E}+03$ \\
\hline ba137m & $1.03 \mathrm{E}+05$ & $1.41 \mathrm{E}+07$ & $1.11 \mathrm{E}+05$ & $1.53 \mathrm{E}+07$ \\
\hline ba139 & $1.39 \mathrm{E}+06$ & $1.91 \mathrm{E}+08$ & $1.42 \mathrm{E}+06$ & $1.97 \mathrm{E}+08$ \\
\hline ba140 & $1.34 \mathrm{E}+06$ & $1.84 \mathrm{E}+08$ & $1.37 \mathrm{E}+06$ & $1.90 \mathrm{E}+08$ \\
\hline ba141 & $1.25 \mathrm{E}+06$ & $1.72 \mathrm{E}+08$ & $1.28 \mathrm{E}+06$ & $1.77 \mathrm{E}+08$ \\
\hline ba142 & $1.17 \mathrm{E}+06$ & $1.60 \mathrm{E}+08$ & $1.20 \mathrm{E}+06$ & $1.67 \mathrm{E}+08$ \\
\hline br83 & $8.86 \mathrm{E}+04$ & $1.21 \mathrm{E}+07$ & $9.60 \mathrm{E}+04$ & $1.33 \mathrm{E}+07$ \\
\hline br84 & $1.48 \mathrm{E}+05$ & $2.03 \mathrm{E}+07$ & $1.63 \mathrm{E}+05$ & $2.26 \mathrm{E}+07$ \\
\hline br84m & $7.08 \mathrm{E}+03$ & $9.71 \mathrm{E}+05$ & $6.57 \mathrm{E}+03$ & $9.11 \mathrm{E}+05$ \\
\hline br85 & $1.96 \mathrm{E}+05$ & $2.68 \mathrm{E}+07$ & $2.19 \mathrm{E}+05$ & $3.04 \mathrm{E}+07$ \\
\hline br87 & $2.84 \mathrm{E}+05$ & $3.89 \mathrm{E}+07$ & $3.21 \mathrm{E}+05$ & $4.45 \mathrm{E}+07$ \\
\hline $\operatorname{cd} 112$ & $0.00 \mathrm{E}+00$ & $0.00 \mathrm{E}+00$ & $0.00 \mathrm{E}+00$ & $0.00 \mathrm{E}+00$ \\
\hline cd114 & $0.00 \mathrm{E}+00$ & $0.00 \mathrm{E}+00$ & $0.00 \mathrm{E}+00$ & $0.00 \mathrm{E}+00$ \\
\hline ce140 & $0.00 \mathrm{E}+00$ & $0.00 \mathrm{E}+00$ & $0.00 \mathrm{E}+00$ & $0.00 \mathrm{E}+00$ \\
\hline ce141 & $1.27 \mathrm{E}+06$ & $1.73 \mathrm{E}+08$ & $1.29 \mathrm{E}+06$ & $1.79 \mathrm{E}+08$ \\
\hline ce142 & $0.00 \mathrm{E}+00$ & $0.00 \mathrm{E}+00$ & $0.00 \mathrm{E}+00$ & $0.00 \mathrm{E}+00$ \\
\hline ce143 & $1.16 \mathrm{E}+06$ & $1.59 \mathrm{E}+08$ & $1.21 \mathrm{E}+06$ & $1.67 \mathrm{E}+08$ \\
\hline ce144 & $9.84 \mathrm{E}+05$ & $1.35 \mathrm{E}+08$ & $1.06 \mathrm{E}+06$ & $1.47 \mathrm{E}+08$ \\
\hline ce145 & $7.94 \mathrm{E}+05$ & $1.09 \mathrm{E}+08$ & $8.20 \mathrm{E}+05$ & $1.14 \mathrm{E}+08$ \\
\hline $\mathrm{cm} 242$ & $6.59 \mathrm{E}+04$ & $9.02 \mathrm{E}+06$ & $4.27 \mathrm{E}+04$ & $5.92 \mathrm{E}+06$ \\
\hline $\mathrm{cm} 243$ & $2.39 \mathrm{E}+01$ & $3.28 \mathrm{E}+03$ & $1.55 \mathrm{E}+01$ & $2.14 \mathrm{E}+03$ \\
\hline $\mathrm{cm} 244$ & $3.70 \mathrm{E}+03$ & $5.07 \mathrm{E}+05$ & $3.06 \mathrm{E}+03$ & $4.24 \mathrm{E}+05$ \\
\hline $\mathrm{cm} 245$ & $4.58 \mathrm{E}-01$ & $6.28 \mathrm{E}+01$ & $3.35 \mathrm{E}-01$ & $4.65 \mathrm{E}+01$ \\
\hline $\mathrm{cm} 246$ & $1.03 \mathrm{E}-01$ & $1.41 \mathrm{E}+01$ & $8.71 \mathrm{E}-02$ & $1.21 \mathrm{E}+01$ \\
\hline $\operatorname{co58}$ & $2.21 \mathrm{E}-13$ & $3.03 \mathrm{E}-11$ & $1.83 \mathrm{E}-13$ & $2.53 \mathrm{E}-11$ \\
\hline $\operatorname{co6} 60$ & $1.11 \mathrm{E}-11$ & $1.52 \mathrm{E}-09$ & $1.23 \mathrm{E}-11$ & $1.71 \mathrm{E}-09$ \\
\hline $\operatorname{cs} 133$ & $0.00 \mathrm{E}+00$ & $0.00 \mathrm{E}+00$ & $0.00 \mathrm{E}+00$ & $0.00 \mathrm{E}+00$ \\
\hline $\operatorname{cs} 134$ & $1.42 \mathrm{E}+05$ & $1.94 \mathrm{E}+07$ & $1.60 \mathrm{E}+05$ & $2.22 \mathrm{E}+07$ \\
\hline $\operatorname{cs} 135$ & $6.09 \mathrm{E}-01$ & $8.34 \mathrm{E}+01$ & $5.91 \mathrm{E}-01$ & $8.19 \mathrm{E}+01$ \\
\hline $\operatorname{cs} 136$ & $3.85 \mathrm{E}+04$ & $5.27 \mathrm{E}+06$ & $3.80 \mathrm{E}+04$ & $5.27 \mathrm{E}+06$ \\
\hline $\operatorname{cs} 137$ & $1.08 \mathrm{E}+05$ & $1.48 \mathrm{E}+07$ & $1.16 \mathrm{E}+05$ & $1.62 \mathrm{E}+07$ \\
\hline $\operatorname{cs} 138$ & $1.46 \mathrm{E}+06$ & $2.01 \mathrm{E}+08$ & $1.49 \mathrm{E}+06$ & $2.06 \mathrm{E}+08$ \\
\hline $\operatorname{cs} 139$ & $1.36 \mathrm{E}+06$ & $1.87 \mathrm{E}+08$ & $1.39 \mathrm{E}+06$ & $1.92 \mathrm{E}+08$ \\
\hline $\operatorname{cs} 140$ & $1.13 \mathrm{E}+06$ & $1.55 \mathrm{E}+08$ & $1.17 \mathrm{E}+06$ & $1.63 \mathrm{E}+08$ \\
\hline cs141 & $9.02 \mathrm{E}+05$ & $1.24 \mathrm{E}+08$ & $9.22 \mathrm{E}+05$ & $1.28 \mathrm{E}+08$ \\
\hline eu151 & $0.00 \mathrm{E}+00$ & $0.00 \mathrm{E}+00$ & $0.00 \mathrm{E}+00$ & $0.00 \mathrm{E}+00$ \\
\hline
\end{tabular}


Table 11 (continued)

\begin{tabular}{|c|c|c|c|c|}
\hline \multirow{2}{*}{ Nuclide } & \multicolumn{2}{|c|}{ MOX core } & \multicolumn{2}{|c|}{ LEU core } \\
\hline & Curies/MTHM & Total curies & Curies/MTHM & Total curies \\
\hline eu153 & $0.00 \mathrm{E}+00$ & $0.00 \mathrm{E}+00$ & $0.00 \mathrm{E}+00$ & $0.00 \mathrm{E}+00$ \\
\hline eu154 & $6.80 \mathrm{E}+03$ & $9.32 \mathrm{E}+05$ & $6.44 \mathrm{E}+03$ & $8.93 \mathrm{E}+05$ \\
\hline eu155 & $4.26 \mathrm{E}+03$ & $5.84 \mathrm{E}+05$ & $4.12 \mathrm{E}+03$ & $5.71 \mathrm{E}+05$ \\
\hline $\operatorname{gd} 154$ & $0.00 \mathrm{E}+00$ & $0.00 \mathrm{E}+00$ & $0.00 \mathrm{E}+00$ & $0.00 \mathrm{E}+00$ \\
\hline $\operatorname{gd} 155$ & $0.00 \mathrm{E}+00$ & $0.00 \mathrm{E}+00$ & $0.00 \mathrm{E}+00$ & $0.00 \mathrm{E}+00$ \\
\hline gd156 & $0.00 \mathrm{E}+00$ & $0.00 \mathrm{E}+00$ & $0.00 \mathrm{E}+00$ & $0.00 \mathrm{E}+00$ \\
\hline gd157 & $0.00 \mathrm{E}+00$ & $0.00 \mathrm{E}+00$ & $0.00 \mathrm{E}+00$ & $0.00 \mathrm{E}+00$ \\
\hline $\operatorname{gd} 158$ & $0.00 \mathrm{E}+00$ & $0.00 \mathrm{E}+00$ & $0.00 \mathrm{E}+00$ & $0.00 \mathrm{E}+00$ \\
\hline i129 & $3.11 \mathrm{E}-02$ & $4.26 \mathrm{E}+00$ & $2.90 \mathrm{E}-02$ & $4.02 \mathrm{E}+00$ \\
\hline i130 & $1.59 \mathrm{E}+04$ & $2.18 \mathrm{E}+06$ & $1.63 \mathrm{E}+04$ & $2.26 \mathrm{E}+06$ \\
\hline i131 & $7.93 \mathrm{E}+05$ & $1.09 \mathrm{E}+08$ & $7.78 \mathrm{E}+05$ & $1.08 \mathrm{E}+08$ \\
\hline i132 & $1.15 \mathrm{E}+06$ & $1.58 \mathrm{E}+08$ & $1.14 \mathrm{E}+06$ & $1.58 \mathrm{E}+08$ \\
\hline i133 & $1.59 \mathrm{E}+06$ & $2.18 \mathrm{E}+08$ & $1.60 \mathrm{E}+06$ & $2.22 \mathrm{E}+08$ \\
\hline i134 & $1.78 \mathrm{E}+06$ & $2.43 \mathrm{E}+08$ & $1.79 \mathrm{E}+06$ & $2.49 \mathrm{E}+08$ \\
\hline i135 & $1.52 \mathrm{E}+06$ & $2.09 \mathrm{E}+08$ & $1.52 \mathrm{E}+06$ & $2.11 \mathrm{E}+08$ \\
\hline $\mathrm{i} 136 \mathrm{~m}$ & $3.59 \mathrm{E}+05$ & $4.92 \mathrm{E}+07$ & $3.49 \mathrm{E}+05$ & $4.83 \mathrm{E}+07$ \\
\hline $\mathrm{kr} 83 \mathrm{~m}$ & $8.79 \mathrm{E}+04$ & $1.20 \mathrm{E}+07$ & $9.54 \mathrm{E}+04$ & $1.32 \mathrm{E}+07$ \\
\hline kr85 & $1.09 \mathrm{E}+04$ & $1.49 \mathrm{E}+06$ & $1.36 \mathrm{E}+04$ & $1.89 \mathrm{E}+06$ \\
\hline $\mathrm{kr} 85 \mathrm{~m}$ & $1.89 \mathrm{E}+05$ & $2.59 \mathrm{E}+07$ & $2.12 \mathrm{E}+05$ & $2.93 \mathrm{E}+07$ \\
\hline kr87 & $3.62 \mathrm{E}+05$ & $4.96 \mathrm{E}+07$ & $4.07 \mathrm{E}+05$ & $5.64 \mathrm{E}+07$ \\
\hline kr88 & $4.78 \mathrm{E}+05$ & $6.54 \mathrm{E}+07$ & $5.40 \mathrm{E}+05$ & $7.48 \mathrm{E}+07$ \\
\hline kr89 & $5.92 \mathrm{E}+05$ & $8.11 \mathrm{E}+07$ & $6.75 \mathrm{E}+05$ & $9.36 \mathrm{E}+07$ \\
\hline kr90 & $6.07 \mathrm{E}+05$ & $8.32 \mathrm{E}+07$ & $7.02 \mathrm{E}+05$ & $9.74 \mathrm{E}+07$ \\
\hline la139 & $0.00 \mathrm{E}+00$ & $0.00 \mathrm{E}+00$ & $0.00 \mathrm{E}+00$ & $0.00 \mathrm{E}+00$ \\
\hline la140 & $1.40 \mathrm{E}+06$ & $1.91 \mathrm{E}+08$ & $1.43 \mathrm{E}+06$ & $1.98 \mathrm{E}+08$ \\
\hline la141 & $1.26 \mathrm{E}+06$ & $1.73 \mathrm{E}+08$ & $1.29 \mathrm{E}+06$ & $1.78 \mathrm{E}+08$ \\
\hline la142 & $1.21 \mathrm{E}+06$ & $1.66 \mathrm{E}+08$ & $1.24 \mathrm{E}+06$ & $1.72 \mathrm{E}+08$ \\
\hline la143 & $1.15 \mathrm{E}+06$ & $1.58 \mathrm{E}+08$ & $1.20 \mathrm{E}+06$ & $1.66 \mathrm{E}+08$ \\
\hline mo100 & $4.43 \mathrm{E}-13$ & $6.06 \mathrm{E}-11$ & $4.78 \mathrm{E}-13$ & $6.63 \mathrm{E}-11$ \\
\hline mo92 & $0.00 \mathrm{E}+00$ & $0.00 \mathrm{E}+00$ & $0.00 \mathrm{E}+00$ & $0.00 \mathrm{E}+00$ \\
\hline mo94 & $0.00 \mathrm{E}+00$ & $0.00 \mathrm{E}+00$ & $0.00 \mathrm{E}+00$ & $0.00 \mathrm{E}+00$ \\
\hline mo95 & $0.00 \mathrm{E}+00$ & $0.00 \mathrm{E}+00$ & $0.00 \mathrm{E}+00$ & $0.00 \mathrm{E}+00$ \\
\hline mo96 & $0.00 \mathrm{E}+00$ & $0.00 \mathrm{E}+00$ & $0.00 \mathrm{E}+00$ & $0.00 \mathrm{E}+00$ \\
\hline mo97 & $0.00 \mathrm{E}+00$ & $0.00 \mathrm{E}+00$ & $0.00 \mathrm{E}+00$ & $0.00 \mathrm{E}+00$ \\
\hline mo98 & $0.00 \mathrm{E}+00$ & $0.00 \mathrm{E}+00$ & $0.00 \mathrm{E}+00$ & $0.00 \mathrm{E}+00$ \\
\hline mo99 & $1.44 \mathrm{E}+06$ & $1.97 \mathrm{E}+08$ & $1.45 \mathrm{E}+06$ & $2.01 \mathrm{E}+08$ \\
\hline nb95 & $1.26 \mathrm{E}+06$ & $1.73 \mathrm{E}+08$ & $1.32 \mathrm{E}+06$ & $1.84 \mathrm{E}+08$ \\
\hline nb97 & $1.31 \mathrm{E}+06$ & $1.80 \mathrm{E}+08$ & $1.34 \mathrm{E}+06$ & $1.86 \mathrm{E}+08$ \\
\hline nb97m & $1.84 \mathrm{E}+03$ & $2.52 \mathrm{E}+05$ & $1.63 \mathrm{E}+03$ & $2.26 \mathrm{E}+05$ \\
\hline nd142 & $0.00 \mathrm{E}+00$ & $0.00 \mathrm{E}+00$ & $0.00 \mathrm{E}+00$ & $0.00 \mathrm{E}+00$ \\
\hline nd143 & $0.00 \mathrm{E}+00$ & $0.00 \mathrm{E}+00$ & $0.00 \mathrm{E}+00$ & $0.00 \mathrm{E}+00$ \\
\hline nd144 & $1.03 \mathrm{E}-09$ & $1.42 \mathrm{E}-07$ & $1.28 \mathrm{E}-09$ & $1.77 \mathrm{E}-07$ \\
\hline nd145 & $0.00 \mathrm{E}+00$ & $0.00 \mathrm{E}+00$ & $0.00 \mathrm{E}+00$ & $0.00 \mathrm{E}+00$ \\
\hline nd146 & $0.00 \mathrm{E}+00$ & $0.00 \mathrm{E}+00$ & $0.00 \mathrm{E}+00$ & $0.00 \mathrm{E}+00$ \\
\hline nd147 & $5.05 \mathrm{E}+05$ & $6.91 \mathrm{E}+07$ & $5.12 \mathrm{E}+05$ & $7.09 \mathrm{E}+07$ \\
\hline nd148 & $0.00 \mathrm{E}+00$ & $0.00 \mathrm{E}+00$ & $0.00 \mathrm{E}+00$ & $0.00 \mathrm{E}+00$ \\
\hline nd150 & $5.76 \mathrm{E}-14$ & $7.89 \mathrm{E}-12$ & $5.85 \mathrm{E}-14$ & $8.11 \mathrm{E}-12$ \\
\hline np237 & $2.30 \mathrm{E}-01$ & $3.15 \mathrm{E}+01$ & $3.15 \mathrm{E}-01$ & $4.37 \mathrm{E}+01$ \\
\hline np238 & $1.92 \mathrm{E}+05$ & $2.63 \mathrm{E}+07$ & $2.74 \mathrm{E}+05$ & $3.80 \mathrm{E}+07$ \\
\hline
\end{tabular}


Table 11 (continued)

\begin{tabular}{|c|c|c|c|c|}
\hline \multirow{2}{*}{ Nuclide } & \multicolumn{2}{|c|}{ MOX core } & \multicolumn{2}{|c|}{ LEU core } \\
\hline & Curies/MTHM & Total curies & Curies/MTHM & Total curies \\
\hline np239 & $1.37 \mathrm{E}+07$ & $1.88 \mathrm{E}+09$ & $1.40 \mathrm{E}+07$ & $\begin{array}{c}1.94 \mathrm{E}+09 \\
\end{array}$ \\
\hline np240 & $6.66 \mathrm{E}+03$ & $9.13 \mathrm{E}+05$ & $7.04 \mathrm{E}+03$ & $9.76 \mathrm{E}+05$ \\
\hline pd105 & $0.00 \mathrm{E}+00$ & $0.00 \mathrm{E}+00$ & $0.00 \mathrm{E}+00$ & $0.00 \mathrm{E}+00$ \\
\hline pd107 & $1.46 \mathrm{E}-01$ & $2.00 \mathrm{E}+01$ & $1.09 \mathrm{E}-01$ & $1.51 \mathrm{E}+01$ \\
\hline pd108 & $0.00 \mathrm{E}+00$ & $0.00 \mathrm{E}+00$ & $0.00 \mathrm{E}+00$ & $0.00 \mathrm{E}+00$ \\
\hline pd110 & $0.00 \mathrm{E}+00$ & $0.00 \mathrm{E}+00$ & $0.00 \mathrm{E}+00$ & $0.00 \mathrm{E}+00$ \\
\hline pm147 & $1.77 \mathrm{E}+05$ & $2.43 \mathrm{E}+07$ & $1.87 \mathrm{E}+05$ & $2.59 \mathrm{E}+07$ \\
\hline pr143 & $1.14 \mathrm{E}+06$ & $1.57 \mathrm{E}+08$ & $1.19 \mathrm{E}+06$ & $1.65 \mathrm{E}+08$ \\
\hline pr144 & $9.90 \mathrm{E}+05$ & $1.36 \mathrm{E}+08$ & $1.06 \mathrm{E}+06$ & $1.48 \mathrm{E}+08$ \\
\hline pr145 & $7.94 \mathrm{E}+05$ & $1.09 \mathrm{E}+08$ & $8.21 \mathrm{E}+05$ & $1.14 \mathrm{E}+08$ \\
\hline pu237 & $3.62 \mathrm{E}+00$ & $4.96 \mathrm{E}+02$ & $4.08 \mathrm{E}+00$ & $5.65 \mathrm{E}+02$ \\
\hline pu238 & $2.20 \mathrm{E}+03$ & $3.02 \mathrm{E}+05$ & $2.75 \mathrm{E}+03$ & $3.81 \mathrm{E}+05$ \\
\hline pu239 & $4.57 \mathrm{E}+02$ & $6.26 \mathrm{E}+04$ & $3.03 \mathrm{E}+02$ & $4.20 \mathrm{E}+04$ \\
\hline pu240 & $8.67 \mathrm{E}+02$ & $1.19 \mathrm{E}+05$ & $4.85 \mathrm{E}+02$ & $6.73 \mathrm{E}+04$ \\
\hline pu241 & $1.71 \mathrm{E}+05$ & $2.35 \mathrm{E}+07$ & $1.09 \mathrm{E}+05$ & $1.52 \mathrm{E}+07$ \\
\hline pu242 & $2.48 \mathrm{E}+00$ & $3.39 \mathrm{E}+02$ & $1.84 \mathrm{E}+00$ & $2.55 \mathrm{E}+02$ \\
\hline pu243 & $3.12 \mathrm{E}+05$ & $4.27 \mathrm{E}+07$ & $2.51 \mathrm{E}+05$ & $3.49 \mathrm{E}+07$ \\
\hline pu244 & $5.85 \mathrm{E}-07$ & $8.02 \mathrm{E}-05$ & $4.91 \mathrm{E}-07$ & $6.81 \mathrm{E}-05$ \\
\hline pu245 & $1.75 \mathrm{E}+00$ & $2.40 \mathrm{E}+02$ & $1.53 \mathrm{E}+00$ & $2.13 \mathrm{E}+02$ \\
\hline $\mathrm{rb} 86$ & $1.55 \mathrm{E}+03$ & $2.13 \mathrm{E}+05$ & $1.99 \mathrm{E}+03$ & $2.75 \mathrm{E}+05$ \\
\hline $\mathrm{rb} 88$ & $4.87 \mathrm{E}+05$ & $6.67 \mathrm{E}+07$ & $5.49 \mathrm{E}+05$ & $7.61 \mathrm{E}+07$ \\
\hline rb89 & $6.40 \mathrm{E}+05$ & $8.76 \mathrm{E}+07$ & $7.23 \mathrm{E}+05$ & $1.00 \mathrm{E}+08$ \\
\hline $\mathrm{rb} 90$ & $6.39 \mathrm{E}+05$ & $8.75 \mathrm{E}+07$ & $7.35 \mathrm{E}+05$ & $1.02 \mathrm{E}+08$ \\
\hline rb90m & $1.30 \mathrm{E}+05$ & $1.79 \mathrm{E}+07$ & $1.36 \mathrm{E}+05$ & $1.89 \mathrm{E}+07$ \\
\hline rb91 & $7.86 \mathrm{E}+05$ & $1.08 \mathrm{E}+08$ & $8.81 \mathrm{E}+05$ & $1.22 \mathrm{E}+08$ \\
\hline rh103m & $1.30 \mathrm{E}+06$ & $1.78 \mathrm{E}+08$ & $1.20 \mathrm{E}+06$ & $1.66 \mathrm{E}+08$ \\
\hline rh105 & $8.78 \mathrm{E}+05$ & $1.20 \mathrm{E}+08$ & $7.64 \mathrm{E}+05$ & $1.06 \mathrm{E}+08$ \\
\hline rh105m & $2.66 \mathrm{E}+05$ & $3.64 \mathrm{E}+07$ & $2.33 \mathrm{E}+05$ & $3.23 \mathrm{E}+07$ \\
\hline rh106 & $6.14 \mathrm{E}+05$ & $8.41 \mathrm{E}+07$ & $4.96 \mathrm{E}+05$ & $6.88 \mathrm{E}+07$ \\
\hline rh107 & $5.41 \mathrm{E}+05$ & $7.42 \mathrm{E}+07$ & $4.52 \mathrm{E}+05$ & $6.27 \mathrm{E}+07$ \\
\hline ru101 & $0.00 \mathrm{E}+00$ & $0.00 \mathrm{E}+00$ & $0.00 \mathrm{E}+00$ & $0.00 \mathrm{E}+00$ \\
\hline ru102 & $0.00 \mathrm{E}+00$ & $0.00 \mathrm{E}+00$ & $0.00 \mathrm{E}+00$ & $0.00 \mathrm{E}+00$ \\
\hline ru103 & $1.31 \mathrm{E}+06$ & $1.80 \mathrm{E}+08$ & $1.21 \mathrm{E}+06$ & $1.68 \mathrm{E}+08$ \\
\hline ru104 & $0.00 \mathrm{E}+00$ & $0.00 \mathrm{E}+00$ & $0.00 \mathrm{E}+00$ & $0.00 \mathrm{E}+00$ \\
\hline ru105 & $9.44 \mathrm{E}+05$ & $1.29 \mathrm{E}+08$ & $8.26 \mathrm{E}+05$ & $1.15 \mathrm{E}+08$ \\
\hline ru106 & $5.62 \mathrm{E}+05$ & $7.71 \mathrm{E}+07$ & $4.46 \mathrm{E}+05$ & $6.19 \mathrm{E}+07$ \\
\hline ru107 & $5.33 \mathrm{E}+05$ & $7.30 \mathrm{E}+07$ & $4.46 \mathrm{E}+05$ & $6.18 \mathrm{E}+07$ \\
\hline sb125 & $9.92 \mathrm{E}+03$ & $1.36 \mathrm{E}+06$ & $8.90 \mathrm{E}+03$ & $1.23 \mathrm{E}+06$ \\
\hline $\mathrm{sb} 127$ & $7.89 \mathrm{E}+04$ & $1.08 \mathrm{E}+07$ & $7.24 \mathrm{E}+04$ & $1.00 \mathrm{E}+07$ \\
\hline sb129 & $2.36 \mathrm{E}+05$ & $3.23 \mathrm{E}+07$ & $2.20 \mathrm{E}+05$ & $3.05 \mathrm{E}+07$ \\
\hline sb130 & $2.25 \mathrm{E}+05$ & $3.09 \mathrm{E}+07$ & $2.21 \mathrm{E}+05$ & $3.06 \mathrm{E}+07$ \\
\hline sb130m & $2.34 \mathrm{E}+05$ & $3.21 \mathrm{E}+07$ & $2.33 \mathrm{E}+05$ & $3.23 \mathrm{E}+07$ \\
\hline sb133 & $4.63 \mathrm{E}+05$ & $6.34 \mathrm{E}+07$ & $4.85 \mathrm{E}+05$ & $6.72 \mathrm{E}+07$ \\
\hline se84 & $1.43 \mathrm{E}+05$ & $1.96 \mathrm{E}+07$ & $1.58 \mathrm{E}+05$ & $2.19 \mathrm{E}+07$ \\
\hline sm147 & $2.01 \mathrm{E}-06$ & $2.75 \mathrm{E}-04$ & $2.29 \mathrm{E}-06$ & $3.17 \mathrm{E}-04$ \\
\hline sm148 & $4.38 \mathrm{E}-11$ & $6.00 \mathrm{E}-09$ & $5.02 \mathrm{E}-11$ & $6.97 \mathrm{E}-09$ \\
\hline sm149 & $0.00 \mathrm{E}+00$ & $0.00 \mathrm{E}+00$ & $0.00 \mathrm{E}+00$ & $0.00 \mathrm{E}+00$ \\
\hline $\operatorname{sm} 150$ & $0.00 \mathrm{E}+00$ & $0.00 \mathrm{E}+00$ & $0.00 \mathrm{E}+00$ & $0.00 \mathrm{E}+00$ \\
\hline $\mathrm{sm} 151$ & $3.57 \mathrm{E}+02$ & $4.89 \mathrm{E}+04$ & $3.11 \mathrm{E}+02$ & $4.32 \mathrm{E}+04$ \\
\hline
\end{tabular}


Table 11 (continued)

\begin{tabular}{|c|c|c|c|c|}
\hline \multirow{2}{*}{ Nuclide } & \multicolumn{2}{|c|}{ MOX core } & \multicolumn{2}{|c|}{ LEU core } \\
\hline & Curies/MTHM & Total curies & Curies/MTHM & Total curies \\
\hline sm152 & $0.00 \mathrm{E}+00$ & $0.00 \mathrm{E}+00$ & $0.00 \mathrm{E}+00$ & $0.00 \mathrm{E}+00$ \\
\hline sm154 & $0.00 \mathrm{E}+00$ & $0.00 \mathrm{E}+00$ & $0.00 \mathrm{E}+00$ & $0.00 \mathrm{E}+00$ \\
\hline sn130 & $1.40 \mathrm{E}+05$ & $1.91 \mathrm{E}+07$ & $1.43 \mathrm{E}+05$ & $1.98 \mathrm{E}+07$ \\
\hline sr89 & $6.60 \mathrm{E}+05$ & $9.04 \mathrm{E}+07$ & $7.49 \mathrm{E}+05$ & $1.04 \mathrm{E}+08$ \\
\hline sr90 & $6.72 \mathrm{E}+04$ & $9.20 \mathrm{E}+06$ & $8.58 \mathrm{E}+04$ & $1.19 \mathrm{E}+07$ \\
\hline sr91 & $8.50 \mathrm{E}+05$ & $1.16 \mathrm{E}+08$ & $9.44 \mathrm{E}+05$ & $1.31 \mathrm{E}+08$ \\
\hline sr92 & $9.31 \mathrm{E}+05$ & $1.27 \mathrm{E}+08$ & $1.02 \mathrm{E}+06$ & $1.41 \mathrm{E}+08$ \\
\hline sr93 & $1.05 \mathrm{E}+06$ & $1.44 \mathrm{E}+08$ & $1.13 \mathrm{E}+06$ & $1.56 \mathrm{E}+08$ \\
\hline sr94 & $1.04 \mathrm{E}+06$ & $1.43 \mathrm{E}+08$ & $1.11 \mathrm{E}+06$ & $1.54 \mathrm{E}+08$ \\
\hline tc101 & $1.36 \mathrm{E}+06$ & $1.86 \mathrm{E}+08$ & $1.34 \mathrm{E}+06$ & $1.86 \mathrm{E}+08$ \\
\hline tc99 & $1.40 \mathrm{E}+01$ & $1.92 \mathrm{E}+03$ & $1.52 \mathrm{E}+01$ & $2.11 \mathrm{E}+03$ \\
\hline tc99m & $1.28 \mathrm{E}+06$ & $1.75 \mathrm{E}+08$ & $1.28 \mathrm{E}+06$ & $1.78 \mathrm{E}+08$ \\
\hline te $125 \mathrm{~m}$ & $2.19 \mathrm{E}+03$ & $3.01 \mathrm{E}+05$ & $1.97 \mathrm{E}+03$ & $2.73 \mathrm{E}+05$ \\
\hline te 127 & $7.73 \mathrm{E}+04$ & $1.06 \mathrm{E}+07$ & $7.07 \mathrm{E}+04$ & $9.81 \mathrm{E}+06$ \\
\hline te $127 \mathrm{~m}$ & $2.19 \mathrm{E}+03$ & $3.00 \mathrm{E}+05$ & $1.85 \mathrm{E}+03$ & $2.56 \mathrm{E}+05$ \\
\hline te 129 & $2.36 \mathrm{E}+05$ & $3.23 \mathrm{E}+07$ & $2.20 \mathrm{E}+05$ & $3.05 \mathrm{E}+07$ \\
\hline te $129 \mathrm{~m}$ & $1.05 \mathrm{E}+02$ & $1.44 \mathrm{E}+04$ & $9.46 \mathrm{E}+01$ & $1.31 \mathrm{E}+04$ \\
\hline te131 & $7.01 \mathrm{E}+05$ & $9.61 \mathrm{E}+07$ & $6.95 \mathrm{E}+05$ & $9.63 \mathrm{E}+07$ \\
\hline te $131 \mathrm{~m}$ & $1.17 \mathrm{E}+05$ & $1.60 \mathrm{E}+07$ & $1.07 \mathrm{E}+05$ & $1.48 \mathrm{E}+07$ \\
\hline te 132 & $1.12 \mathrm{E}+06$ & $1.53 \mathrm{E}+08$ & $1.11 \mathrm{E}+06$ & $1.54 \mathrm{E}+08$ \\
\hline te133 & $9.08 \mathrm{E}+05$ & $1.24 \mathrm{E}+08$ & $9.22 \mathrm{E}+05$ & $1.28 \mathrm{E}+08$ \\
\hline te $133 \mathrm{~m}$ & $6.65 \mathrm{E}+05$ & $9.11 \mathrm{E}+07$ & $6.71 \mathrm{E}+05$ & $9.31 \mathrm{E}+07$ \\
\hline te134 & $1.38 \mathrm{E}+06$ & $1.89 \mathrm{E}+08$ & $1.43 \mathrm{E}+06$ & $1.98 \mathrm{E}+08$ \\
\hline $\mathrm{u} 234$ & $1.09 \mathrm{E}+00$ & $1.49 \mathrm{E}+02$ & $1.50 \mathrm{E}+00$ & $2.08 \mathrm{E}+02$ \\
\hline u235 & $2.55 \mathrm{E}-02$ & $3.49 \mathrm{E}+00$ & $3.14 \mathrm{E}-02$ & $4.36 \mathrm{E}+00$ \\
\hline u236 & $2.30 \mathrm{E}-01$ & $3.15 \mathrm{E}+01$ & $3.40 \mathrm{E}-01$ & $4.72 \mathrm{E}+01$ \\
\hline u237 & $4.37 \mathrm{E}+05$ & $5.98 \mathrm{E}+07$ & $5.95 \mathrm{E}+05$ & $8.25 \mathrm{E}+07$ \\
\hline u238 & $3.14 \mathrm{E}-01$ & $4.31 \mathrm{E}+01$ & $3.13 \mathrm{E}-01$ & $4.35 \mathrm{E}+01$ \\
\hline u239 & $1.37 \mathrm{E}+07$ & $1.88 \mathrm{E}+09$ & $1.41 \mathrm{E}+07$ & $1.95 \mathrm{E}+09$ \\
\hline xe131m & $1.04 \mathrm{E}+04$ & $1.42 \mathrm{E}+06$ & $1.02 \mathrm{E}+04$ & $1.41 \mathrm{E}+06$ \\
\hline xe133 & $1.54 \mathrm{E}+06$ & $2.11 \mathrm{E}+08$ & $1.54 \mathrm{E}+06$ & $2.14 \mathrm{E}+08$ \\
\hline xe133m & $2.07 \mathrm{E}+04$ & $2.83 \mathrm{E}+06$ & $2.03 \mathrm{E}+04$ & $2.81 \mathrm{E}+06$ \\
\hline xe135 & $5.14 \mathrm{E}+05$ & $7.04 \mathrm{E}+07$ & $4.61 \mathrm{E}+05$ & $6.39 \mathrm{E}+07$ \\
\hline xe135m & $2.50 \mathrm{E}+05$ & $3.43 \mathrm{E}+07$ & $2.41 \mathrm{E}+05$ & $3.34 \mathrm{E}+07$ \\
\hline xe137 & $1.43 \mathrm{E}+06$ & $1.96 \mathrm{E}+08$ & $1.43 \mathrm{E}+06$ & $1.99 \mathrm{E}+08$ \\
\hline xe138 & $1.33 \mathrm{E}+06$ & $1.83 \mathrm{E}+08$ & $1.36 \mathrm{E}+06$ & $1.89 \mathrm{E}+08$ \\
\hline xe139 & $9.64 \mathrm{E}+05$ & $1.32 \mathrm{E}+08$ & $1.01 \mathrm{E}+06$ & $1.39 \mathrm{E}+08$ \\
\hline xe140 & $6.40 \mathrm{E}+05$ & $8.76 \mathrm{E}+07$ & $6.82 \mathrm{E}+05$ & $9.46 \mathrm{E}+07$ \\
\hline y90 & $6.92 \mathrm{E}+04$ & $9.49 \mathrm{E}+06$ & $8.87 \mathrm{E}+04$ & $1.23 \mathrm{E}+07$ \\
\hline y91 & $8.70 \mathrm{E}+05$ & $1.19 \mathrm{E}+08$ & $9.71 \mathrm{E}+05$ & $1.35 \mathrm{E}+08$ \\
\hline $\mathrm{y} 91 \mathrm{~m}$ & $4.87 \mathrm{E}+05$ & $6.67 \mathrm{E}+07$ & $5.40 \mathrm{E}+05$ & $7.49 \mathrm{E}+07$ \\
\hline y92 & $9.41 \mathrm{E}+05$ & $1.29 \mathrm{E}+08$ & $1.03 \mathrm{E}+06$ & $1.43 \mathrm{E}+08$ \\
\hline y93 & $1.08 \mathrm{E}+06$ & $1.48 \mathrm{E}+08$ & $1.16 \mathrm{E}+06$ & $1.60 \mathrm{E}+08$ \\
\hline y94 & $1.15 \mathrm{E}+06$ & $1.58 \mathrm{E}+08$ & $1.22 \mathrm{E}+06$ & $1.69 \mathrm{E}+08$ \\
\hline y95 & $1.21 \mathrm{E}+06$ & $1.66 \mathrm{E}+08$ & $1.27 \mathrm{E}+06$ & $1.76 \mathrm{E}+08$ \\
\hline y96 & $7.16 \mathrm{E}+05$ & $9.81 \mathrm{E}+07$ & $7.62 \mathrm{E}+05$ & $1.06 \mathrm{E}+08$ \\
\hline zr95 & $1.26 \mathrm{E}+06$ & $1.72 \mathrm{E}+08$ & $1.32 \mathrm{E}+06$ & $1.83 \mathrm{E}+08$ \\
\hline zr97 & $1.30 \mathrm{E}+06$ & $1.78 \mathrm{E}+08$ & $1.33 \mathrm{E}+06$ & $1.84 \mathrm{E}+08$ \\
\hline
\end{tabular}


Figure 16 graphically presents the MOX/LEU curie ratios of the total core data presented in Table 11.
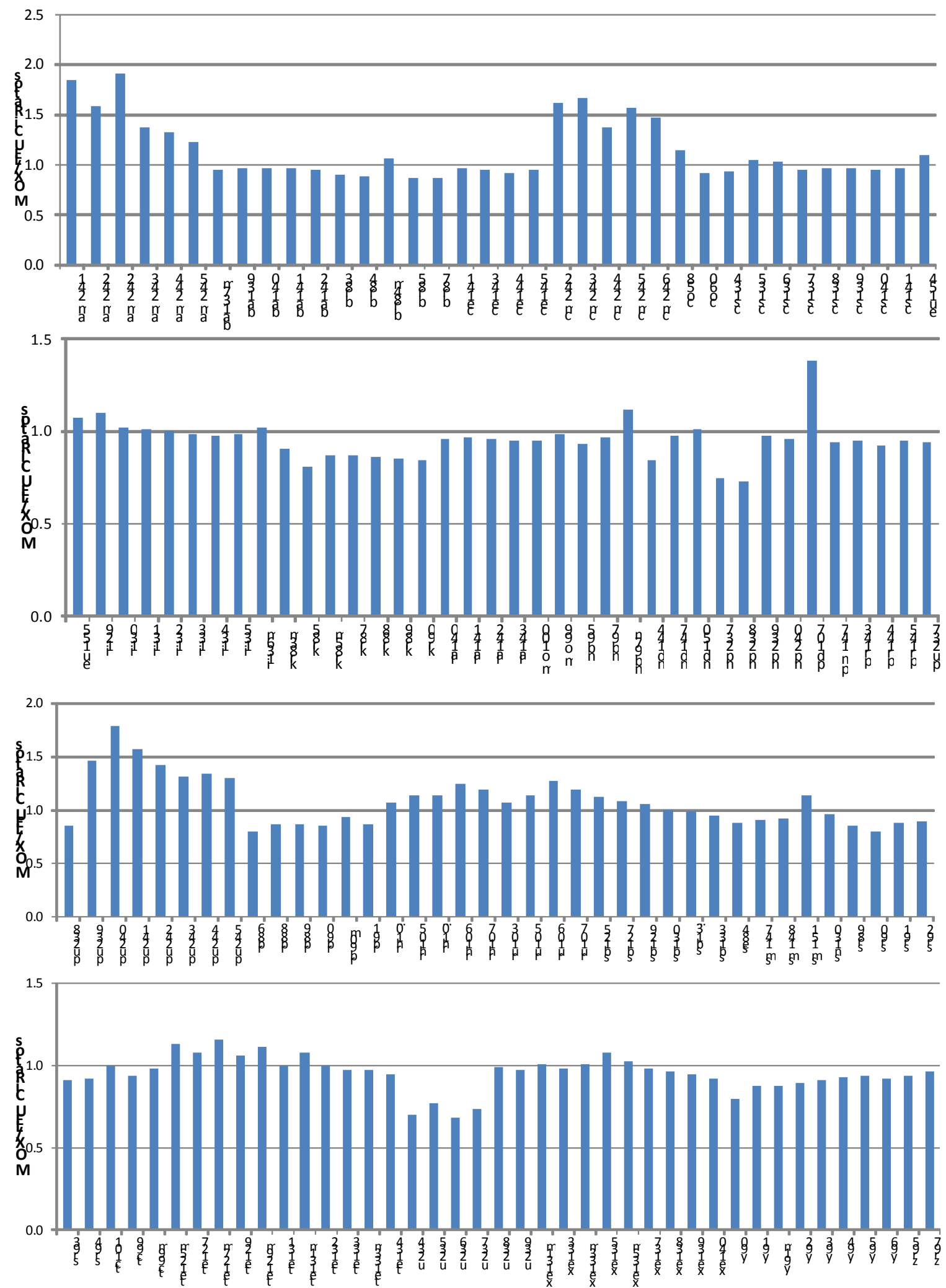

Figure 16. BFN MOX/LEU core-average curie ratios. 


\subsection{Decay Heat}

SQN

The total EOC decay heat trajectories were calculated for each burnup group of each of the SQN MOX and LEU assembly types. This was accomplished by taking the fuel composition at the burnup step closest to the average burnup of each group and simulating a 100-year decay period. The group-average burnups were not exactly the same as those of any particular burnup step, and the next higher burnup value was chosen as the starting point for the decays. Figure 17 presents a comparison of the individual decay heat trajectories, in W/MTHM, of the fuel assemblies in the SQN equilibrium LEU core over a 100 -year period (expressed in days) following discharge from the core. Due to the proprietary nature of the fuel rod loadings, the enrichments have been replaced with letter designations (A, B, etc.). The numerical values are summarized in Table 12. Figure 18, Table 13, and Figure 19, Table 14 present similar comparisons of the individual decay heat trajectories of the LEU and MOX fuel assemblies in the SQN equilibrium MOX core over a 100-year period following discharge from the core. (In the tables and figures, an LEU fuel assembly identification is preceded by an "L." A MOX fuel assembly identification is preceded by an "M." The three burnup classes, low, medium, and high, are designated b1, b2, and b3, respectively.)

The total EOC decay heat trajectories, in watts, for the SQN MOX and LEU cores were obtained by creating new TRITON ft71001 nuclide concentration files for the MOX and LEU cores using the core weighting fractions shown in Table 1 and Table 2, respectively. The new ft71001 files contain the nuclide concentrations of each assembly type, at the burnup step with the burnup closest to the average burnup of a burnup class weighted by their core fractions. The total core average decay heat as a function of time was then calculated by ORIGEN using the core-average set of nuclide concentrations in the new $\mathrm{ft} 71001$ files.

Figure 20 presents the core total decay heat curves decayed for a period of 1 year. The decay heat data are reproduced in tabular form in Table 15. 


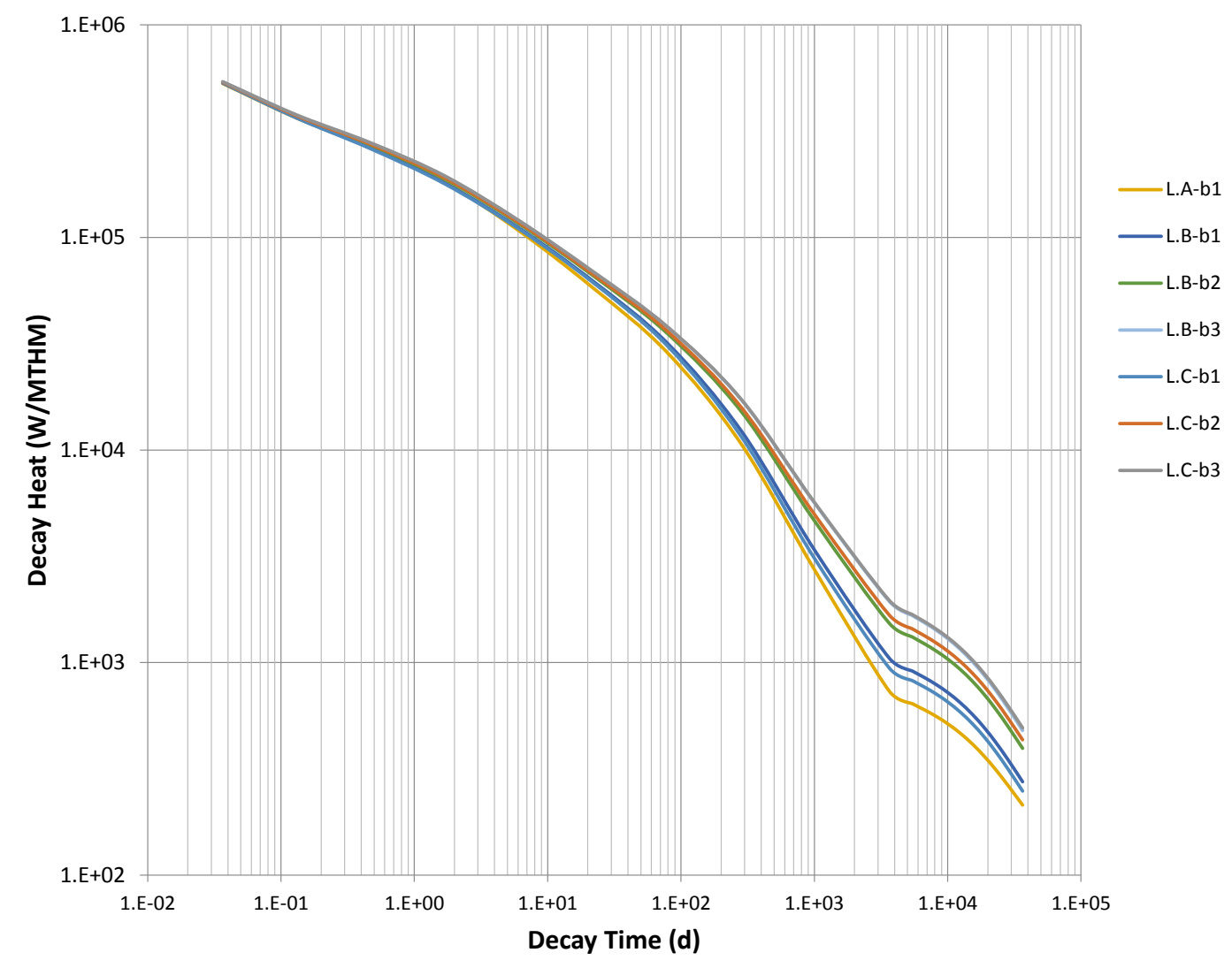

Figure 17. Comparison of assembly decay heat trajectories in the SQN equilibrium LEU core - 100 years.

Table 12. Decay heat (W/MTHM) for the fuel assemblies in the SQN LEU core

\begin{tabular}{|c|c|c|c|c|c|c|c|}
\hline Time (d) & LA-b1 & LB-b1 & LB-b2 & LB-b3 & LC-b1 & LC-b2 & LC-b3 \\
\hline $0.00 \mathrm{E}+00$ & $2.36 \mathrm{E}+06$ & $2.40 \mathrm{E}+06$ & $2.38 \mathrm{E}+06$ & $2.37 \mathrm{E}+06$ & $2.41 \mathrm{E}+06$ & $2.38 \mathrm{E}+06$ & $2.37 \mathrm{E}+06$ \\
\hline $3.65 \mathrm{E}-02$ & $5.31 \mathrm{E}+05$ & $5.33 \mathrm{E}+05$ & $5.36 \mathrm{E}+05$ & $5.39 \mathrm{E}+05$ & $5.34 \mathrm{E}+05$ & $5.38 \mathrm{E}+05$ & $5.40 \mathrm{E}+05$ \\
\hline $1.31 \mathrm{E}-01$ & $3.65 \mathrm{E}+05$ & $3.66 \mathrm{E}+05$ & $3.71 \mathrm{E}+05$ & $3.76 \mathrm{E}+05$ & $3.65 \mathrm{E}+05$ & $3.74 \mathrm{E}+05$ & $3.77 \mathrm{E}+05$ \\
\hline $4.72 \mathrm{E}-01$ & $2.66 \mathrm{E}+05$ & $2.63 \mathrm{E}+05$ & $2.72 \mathrm{E}+05$ & $2.78 \mathrm{E}+05$ & $2.62 \mathrm{E}+05$ & $2.74 \mathrm{E}+05$ & $2.78 \mathrm{E}+05$ \\
\hline $1.70 \mathrm{E}+00$ & $1.82 \mathrm{E}+05$ & $1.80 \mathrm{E}+05$ & $1.88 \mathrm{E}+05$ & $1.94 \mathrm{E}+05$ & $1.79 \mathrm{E}+05$ & $1.91 \mathrm{E}+05$ & $1.95 \mathrm{E}+05$ \\
\hline $6.09 \mathrm{E}+00$ & $1.08 \mathrm{E}+05$ & $1.11 \mathrm{E}+05$ & $1.16 \mathrm{E}+05$ & $1.20 \mathrm{E}+05$ & $1.10 \mathrm{E}+05$ & $1.18 \mathrm{E}+05$ & $1.20 \mathrm{E}+05$ \\
\hline $2.19 \mathrm{E}+01$ & $5.80 \mathrm{E}+04$ & $6.23 \mathrm{E}+04$ & $6.61 \mathrm{E}+04$ & $6.89 \mathrm{E}+04$ & $6.14 \mathrm{E}+04$ & $6.72 \mathrm{E}+04$ & $6.91 \mathrm{E}+04$ \\
\hline $7.87 \mathrm{E}+01$ & $2.88 \mathrm{E}+04$ & $3.19 \mathrm{E}+04$ & $3.54 \mathrm{E}+04$ & $3.80 \mathrm{E}+04$ & $3.10 \mathrm{E}+04$ & $3.64 \mathrm{E}+04$ & $3.81 \mathrm{E}+04$ \\
\hline $2.83 \mathrm{E}+02$ & $1.07 \mathrm{E}+04$ & $1.23 \mathrm{E}+04$ & $1.52 \mathrm{E}+04$ & $1.73 \mathrm{E}+04$ & $1.16 \mathrm{E}+04$ & $1.59 \mathrm{E}+04$ & $1.73 \mathrm{E}+04$ \\
\hline $1.02 \mathrm{E}+03$ & $2.70 \mathrm{E}+03$ & $3.35 \mathrm{E}+03$ & $4.59 \mathrm{E}+03$ & $5.58 \mathrm{E}+03$ & $3.06 \mathrm{E}+03$ & $4.93 \mathrm{E}+03$ & $5.60 \mathrm{E}+03$ \\
\hline $3.65 \mathrm{E}+03$ & $7.35 \mathrm{E}+02$ & $1.05 \mathrm{E}+03$ & $1.53 \mathrm{E}+03$ & $1.94 \mathrm{E}+03$ & $9.44 \mathrm{E}+02$ & $1.67 \mathrm{E}+03$ & $1.96 \mathrm{E}+03$ \\
\hline $5.48 \mathrm{E}+03$ & $6.40 \mathrm{E}+02$ & $9.10 \mathrm{E}+02$ & $1.32 \mathrm{E}+03$ & $1.66 \mathrm{E}+03$ & $8.21 \mathrm{E}+02$ & $1.44 \mathrm{E}+03$ & $1.68 \mathrm{E}+03$ \\
\hline $6.73 \mathrm{E}+03$ & $5.99 \mathrm{E}+02$ & $8.49 \mathrm{E}+02$ & $1.22 \mathrm{E}+03$ & $1.54 \mathrm{E}+03$ & $7.67 \mathrm{E}+02$ & $1.34 \mathrm{E}+03$ & $1.56 \mathrm{E}+03$ \\
\hline $8.26 \mathrm{E}+03$ & $5.58 \mathrm{E}+02$ & $7.87 \mathrm{E}+02$ & $1.13 \mathrm{E}+03$ & $1.42 \mathrm{E}+03$ & $7.10 \mathrm{E}+02$ & $1.24 \mathrm{E}+03$ & $1.43 \mathrm{E}+03$ \\
\hline $1.01 \mathrm{E}+04$ & $5.13 \mathrm{E}+02$ & $7.20 \mathrm{E}+02$ & $1.03 \mathrm{E}+03$ & $1.29 \mathrm{E}+03$ & $6.50 \mathrm{E}+02$ & $1.13 \mathrm{E}+03$ & $1.31 \mathrm{E}+03$ \\
\hline $1.24 \mathrm{E}+04$ & $4.66 \mathrm{E}+02$ & $6.48 \mathrm{E}+02$ & $9.28 \mathrm{E}+02$ & $1.16 \mathrm{E}+03$ & $5.85 \mathrm{E}+02$ & $1.01 \mathrm{E}+03$ & $1.17 \mathrm{E}+03$ \\
\hline $1.53 \mathrm{E}+04$ & $4.15 \mathrm{E}+02$ & $5.73 \mathrm{E}+02$ & $8.19 \mathrm{E}+02$ & $1.02 \mathrm{E}+03$ & $5.18 \mathrm{E}+02$ & $8.95 \mathrm{E}+02$ & $1.03 \mathrm{E}+03$ \\
\hline $1.88 \mathrm{E}+04$ & $3.64 \mathrm{E}+02$ & $4.96 \mathrm{E}+02$ & $7.09 \mathrm{E}+02$ & $8.75 \mathrm{E}+02$ & $4.48 \mathrm{E}+02$ & $7.75 \mathrm{E}+02$ & $8.89 \mathrm{E}+02$ \\
\hline $2.30 \mathrm{E}+04$ & $3.13 \mathrm{E}+02$ & $4.21 \mathrm{E}+02$ & $6.02 \mathrm{E}+02$ & $7.39 \mathrm{E}+02$ & $3.80 \mathrm{E}+02$ & $6.59 \mathrm{E}+02$ & $7.53 \mathrm{E}+02$ \\
\hline $2.83 \mathrm{E}+04$ & $2.66 \mathrm{E}+02$ & $3.51 \mathrm{E}+02$ & $5.02 \mathrm{E}+02$ & $6.14 \mathrm{E}+02$ & $3.17 \mathrm{E}+02$ & $5.50 \mathrm{E}+02$ & $6.28 \mathrm{E}+02$ \\
\hline $3.47 \mathrm{E}+04$ & $2.23 \mathrm{E}+02$ & $2.89 \mathrm{E}+02$ & $4.14 \mathrm{E}+02$ & $5.04 \mathrm{E}+02$ & $2.61 \mathrm{E}+02$ & $4.55 \mathrm{E}+02$ & $5.17 \mathrm{E}+02$ \\
\hline $3.65 \mathrm{E}+04$ & $2.14 \mathrm{E}+02$ & $2.75 \mathrm{E}+02$ & $3.94 \mathrm{E}+02$ & $4.80 \mathrm{E}+02$ & $2.49 \mathrm{E}+02$ & $4.33 \mathrm{E}+02$ & $4.93 \mathrm{E}+02$ \\
\hline
\end{tabular}




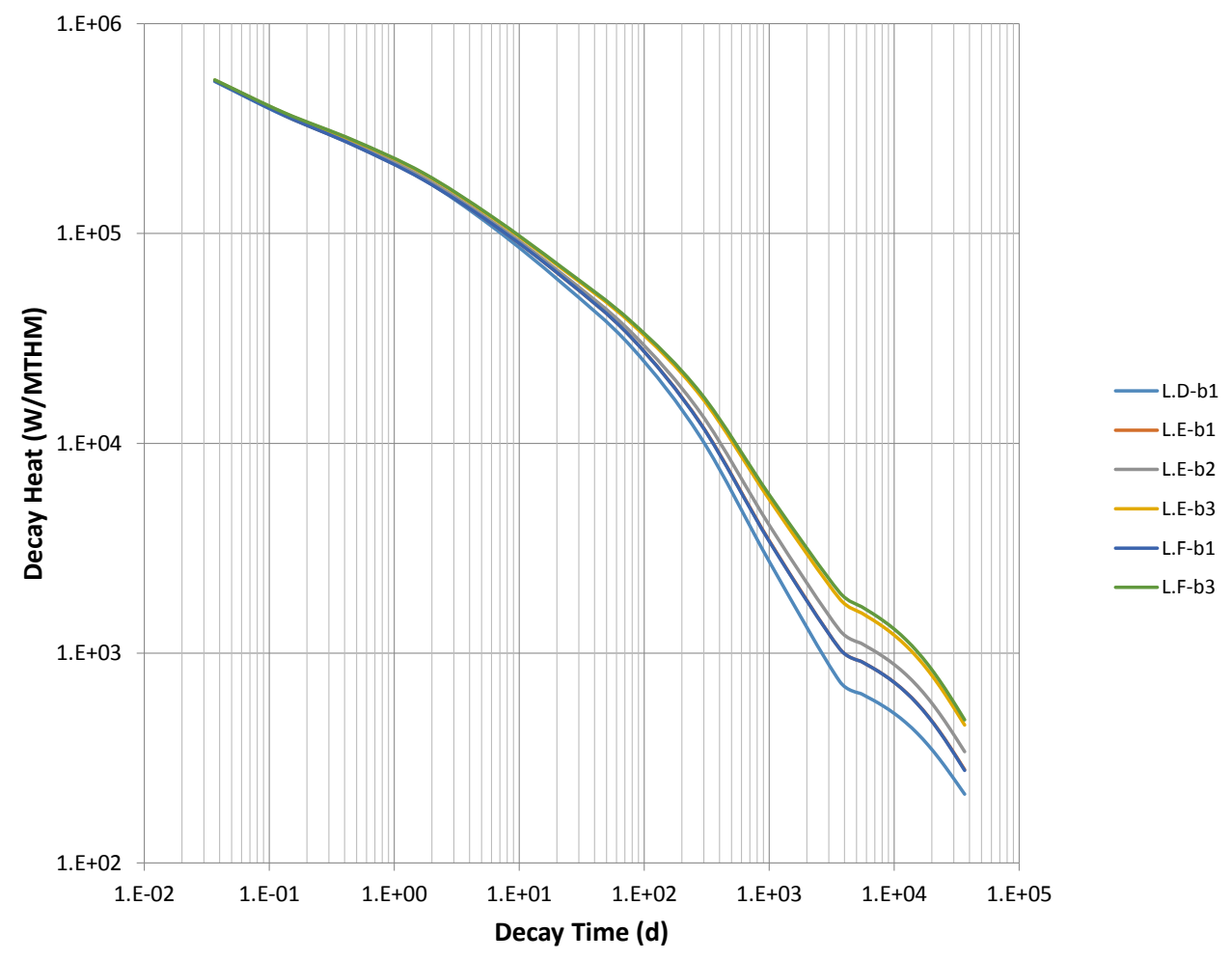

Figure 18. Comparison of assembly decay heat trajectories in the SQN equilibrium MOX core - 100 years, part A.

Table 13. Decay heat (W/MTHM) for the fuel assemblies in the SQN MOX core - part A

\begin{tabular}{||c||c|c|c|c|c|c||}
\hline Time (d) & LD-b1 & LE-b1 & LE-b2 & LE-b3 & LF-b1 & LF-b3 \\
\hline \hline $0.00 \mathrm{E}+00$ & $2.36 \mathrm{E}+06$ & $2.40 \mathrm{E}+06$ & $2.38 \mathrm{E}+06$ & $2.37 \mathrm{E}+06$ & $2.40 \mathrm{E}+06$ & $2.37 \mathrm{E}+06$ \\
\hline $3.65 \mathrm{E}-02$ & $5.31 \mathrm{E}+05$ & $5.33 \mathrm{E}+05$ & $5.35 \mathrm{E}+05$ & $5.39 \mathrm{E}+05$ & $5.33 \mathrm{E}+05$ & $5.40 \mathrm{E}+05$ \\
\hline $1.31 \mathrm{E}-01$ & $3.65 \mathrm{E}+05$ & $3.66 \mathrm{E}+05$ & $3.69 \mathrm{E}+05$ & $3.75 \mathrm{E}+05$ & $3.66 \mathrm{E}+05$ & $3.76 \mathrm{E}+05$ \\
\hline $4.72 \mathrm{E}-01$ & $2.65 \mathrm{E}+05$ & $2.64 \mathrm{E}+05$ & $2.68 \mathrm{E}+05$ & $2.77 \mathrm{E}+05$ & $2.63 \mathrm{E}+05$ & $2.78 \mathrm{E}+05$ \\
\hline $1.70 \mathrm{E}+00$ & $1.82 \mathrm{E}+05$ & $1.81 \mathrm{E}+05$ & $1.85 \mathrm{E}+05$ & $1.93 \mathrm{E}+05$ & $1.81 \mathrm{E}+05$ & $1.95 \mathrm{E}+05$ \\
\hline $6.09 \mathrm{E}+00$ & $1.08 \mathrm{E}+05$ & $1.11 \mathrm{E}+05$ & $1.14 \mathrm{E}+05$ & $1.19 \mathrm{E}+05$ & $1.11 \mathrm{E}+05$ & $1.20 \mathrm{E}+05$ \\
\hline $2.19 \mathrm{E}+01$ & $5.80 \mathrm{E}+04$ & $6.23 \mathrm{E}+04$ & $6.42 \mathrm{E}+04$ & $6.80 \mathrm{E}+04$ & $6.23 \mathrm{E}+04$ & $6.89 \mathrm{E}+04$ \\
\hline $7.87 \mathrm{E}+01$ & $2.88 \mathrm{E}+04$ & $3.19 \mathrm{E}+04$ & $3.38 \mathrm{E}+04$ & $3.73 \mathrm{E}+04$ & $3.19 \mathrm{E}+04$ & $3.80 \mathrm{E}+04$ \\
\hline $2.83 \mathrm{E}+02$ & $1.07 \mathrm{E}+04$ & $1.24 \mathrm{E}+04$ & $1.39 \mathrm{E}+04$ & $1.68 \mathrm{E}+04$ & $1.24 \mathrm{E}+04$ & $1.73 \mathrm{E}+04$ \\
\hline $1.02 \mathrm{E}+03$ & $2.69 \mathrm{E}+03$ & $3.38 \mathrm{E}+03$ & $4.00 \mathrm{E}+03$ & $5.32 \mathrm{E}+03$ & $3.35 \mathrm{E}+03$ & $5.60 \mathrm{E}+03$ \\
\hline $3.65 \mathrm{E}+03$ & $7.35 \mathrm{E}+02$ & $1.05 \mathrm{E}+03$ & $1.29 \mathrm{E}+03$ & $1.82 \mathrm{E}+03$ & $1.05 \mathrm{E}+03$ & $1.95 \mathrm{E}+03$ \\
\hline $5.48 \mathrm{E}+03$ & $6.40 \mathrm{E}+02$ & $9.12 \mathrm{E}+02$ & $1.11 \mathrm{E}+03$ & $1.55 \mathrm{E}+03$ & $9.11 \mathrm{E}+02$ & $1.67 \mathrm{E}+03$ \\
\hline $6.73 \mathrm{E}+03$ & $6.00 \mathrm{E}+02$ & $8.51 \mathrm{E}+02$ & $1.03 \mathrm{E}+03$ & $1.44 \mathrm{E}+03$ & $8.50 \mathrm{E}+02$ & $1.54 \mathrm{E}+03$ \\
\hline $8.26 \mathrm{E}+03$ & $5.58 \mathrm{E}+02$ & $7.89 \mathrm{E}+02$ & $9.57 \mathrm{E}+02$ & $1.33 \mathrm{E}+03$ & $7.88 \mathrm{E}+02$ & $1.42 \mathrm{E}+03$ \\
\hline $1.01 \mathrm{E}+04$ & $5.14 \mathrm{E}+02$ & $7.22 \mathrm{E}+02$ & $8.75 \mathrm{E}+02$ & $1.21 \mathrm{E}+03$ & $7.21 \mathrm{E}+02$ & $1.29 \mathrm{E}+03$ \\
\hline $1.24 \mathrm{E}+04$ & $4.66 \mathrm{E}+02$ & $6.51 \mathrm{E}+02$ & $7.88 \mathrm{E}+02$ & $1.08 \mathrm{E}+03$ & $6.49 \mathrm{E}+02$ & $1.16 \mathrm{E}+03$ \\
\hline $1.53 \mathrm{E}+04$ & $4.15 \mathrm{E}+02$ & $5.76 \mathrm{E}+02$ & $6.97 \mathrm{E}+02$ & $9.53 \mathrm{E}+02$ & $5.74 \mathrm{E}+02$ & $1.02 \mathrm{E}+03$ \\
\hline $1.88 \mathrm{E}+04$ & $3.64 \mathrm{E}+02$ & $4.99 \mathrm{E}+02$ & $6.05 \mathrm{E}+02$ & $8.23 \mathrm{E}+02$ & $4.97 \mathrm{E}+02$ & $8.77 \mathrm{E}+02$ \\
\hline $2.30 \mathrm{E}+04$ & $3.13 \mathrm{E}+02$ & $4.24 \mathrm{E}+02$ & $5.14 \mathrm{E}+02$ & $6.96 \mathrm{E}+02$ & $4.22 \mathrm{E}+02$ & $7.41 \mathrm{E}+02$ \\
\hline $2.83 \mathrm{E}+04$ & $2.65 \mathrm{E}+02$ & $3.55 \mathrm{E}+02$ & $4.30 \mathrm{E}+02$ & $5.80 \mathrm{E}+02$ & $3.52 \mathrm{E}+02$ & $6.16 \mathrm{E}+02$ \\
\hline $3.47 \mathrm{E}+04$ & $2.23 \mathrm{E}+02$ & $2.93 \mathrm{E}+02$ & $3.56 \mathrm{E}+02$ & $4.77 \mathrm{E}+02$ & $2.90 \mathrm{E}+02$ & $5.06 \mathrm{E}+02$ \\
\hline $3.65 \mathrm{E}+04$ & $2.13 \mathrm{E}+02$ & $2.79 \mathrm{E}+02$ & $3.39 \mathrm{E}+02$ & $4.54 \mathrm{E}+02$ & $2.76 \mathrm{E}+02$ & $4.82 \mathrm{E}+02$ \\
\hline
\end{tabular}




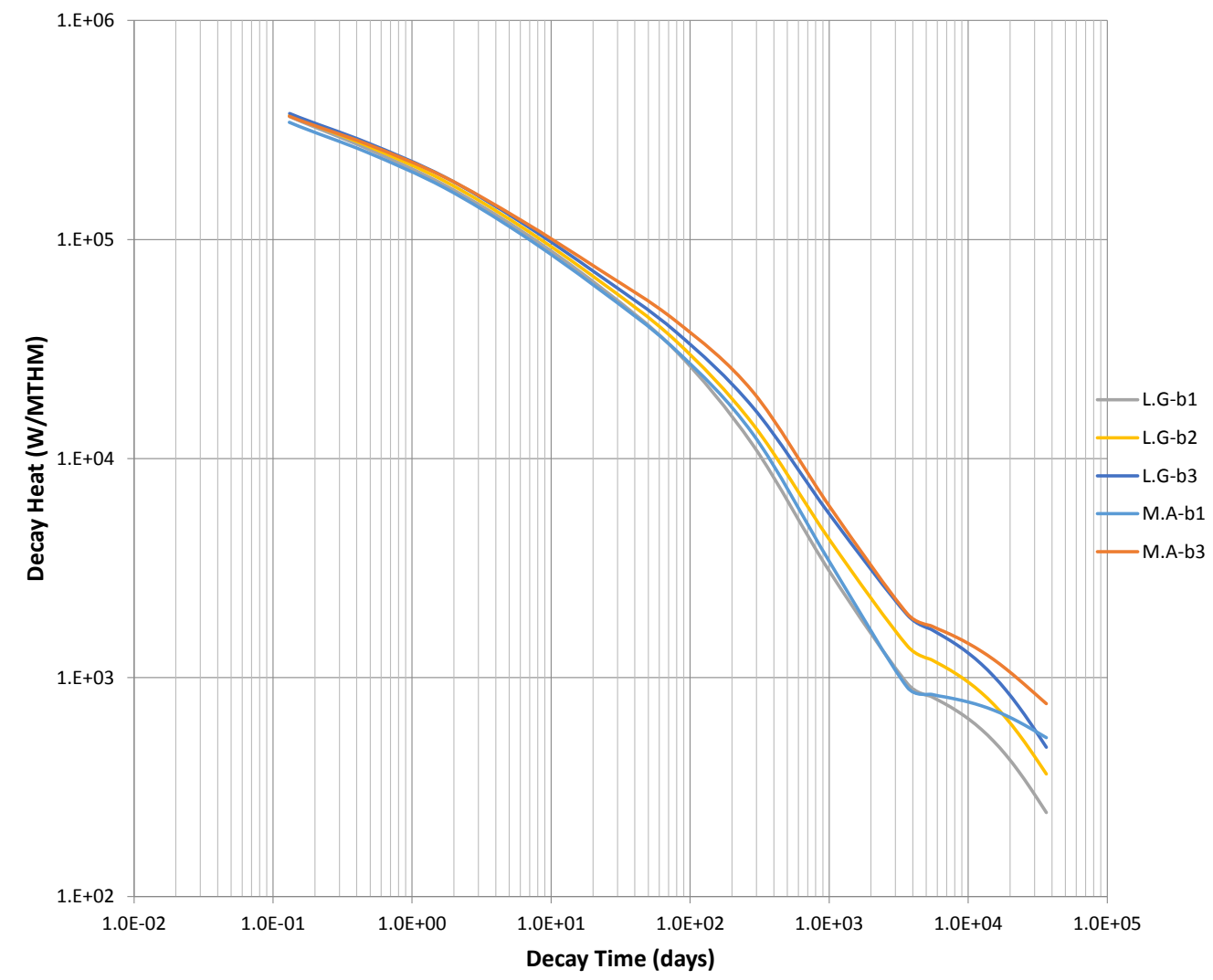

Figure 19. Comparison of assembly decay heat trajectories in the SQN equilibrium MOX core - 100 years, part B.

Table 14. Decay heat (W/MTHM) for the fuel assemblies in the SQN MOX core - part B

\begin{tabular}{|c|c|c|c|c|c||}
\hline \hline Time (d) & LG-b1 & LG-b2 & LG-b3 & MA-b1 & MA-b3 \\
\hline \hline $0.00 \mathrm{E}+00$ & $2.41 \mathrm{E}+06$ & $2.39 \mathrm{E}+06$ & $2.37 \mathrm{E}+06$ & $2.22 \mathrm{E}+06$ & $2.30 \mathrm{E}+06$ \\
\hline $3.65 \mathrm{E}-02$ & $5.33 \mathrm{E}+05$ & $5.36 \mathrm{E}+05$ & $5.39 \mathrm{E}+05$ & $4.98 \mathrm{E}+05$ & $5.21 \mathrm{E}+05$ \\
\hline $1.31 \mathrm{E}-01$ & $3.64 \mathrm{E}+05$ & $3.70 \mathrm{E}+05$ & $3.76 \mathrm{E}+05$ & $3.43 \mathrm{E}+05$ & $3.65 \mathrm{E}+05$ \\
\hline $4.72 \mathrm{E}-01$ & $2.60 \mathrm{E}+05$ & $2.68 \mathrm{E}+05$ & $2.77 \mathrm{E}+05$ & $2.51 \mathrm{E}+05$ & $2.73 \mathrm{E}+05$ \\
\hline $1.70 \mathrm{E}+00$ & $1.78 \mathrm{E}+05$ & $1.86 \mathrm{E}+05$ & $1.93 \mathrm{E}+05$ & $1.73 \mathrm{E}+05$ & $1.93 \mathrm{E}+05$ \\
\hline $6.09 \mathrm{E}+00$ & $1.10 \mathrm{E}+05$ & $1.15 \mathrm{E}+05$ & $1.20 \mathrm{E}+05$ & $1.06 \mathrm{E}+05$ & $1.22 \mathrm{E}+05$ \\
\hline $2.19 \mathrm{E}+01$ & $6.15 \mathrm{E}+04$ & $6.52 \mathrm{E}+04$ & $6.89 \mathrm{E}+04$ & $5.96 \mathrm{E}+04$ & $7.33 \mathrm{E}+04$ \\
\hline $7.87 \mathrm{E}+01$ & $3.10 \mathrm{E}+04$ & $3.45 \mathrm{E}+04$ & $3.79 \mathrm{E}+04$ & $3.13 \mathrm{E}+04$ & $4.25 \mathrm{E}+04$ \\
\hline $2.83 \mathrm{E}+02$ & $1.15 \mathrm{E}+04$ & $1.44 \mathrm{E}+04$ & $1.71 \mathrm{E}+04$ & $1.30 \mathrm{E}+04$ & $2.02 \mathrm{E}+04$ \\
\hline $1.02 \mathrm{E}+03$ & $3.02 \mathrm{E}+03$ & $4.22 \mathrm{E}+03$ & $5.51 \mathrm{E}+03$ & $3.35 \mathrm{E}+03$ & $6.00 \mathrm{E}+03$ \\
\hline $3.65 \mathrm{E}+03$ & $9.38 \mathrm{E}+02$ & $1.40 \mathrm{E}+03$ & $1.93 \mathrm{E}+03$ & $9.03 \mathrm{E}+02$ & $1.96 \mathrm{E}+03$ \\
\hline $5.48 \mathrm{E}+03$ & $8.16 \mathrm{E}+02$ & $1.21 \mathrm{E}+03$ & $1.65 \mathrm{E}+03$ & $8.38 \mathrm{E}+02$ & $1.72 \mathrm{E}+03$ \\
\hline $6.73 \mathrm{E}+03$ & $7.62 \mathrm{E}+02$ & $1.12 \mathrm{E}+03$ & $1.53 \mathrm{E}+03$ & $8.18 \mathrm{E}+02$ & $1.62 \mathrm{E}+03$ \\
\hline $8.26 \mathrm{E}+03$ & $7.05 \mathrm{E}+02$ & $1.04 \mathrm{E}+03$ & $1.41 \mathrm{E}+03$ & $7.97 \mathrm{E}+02$ & $1.53 \mathrm{E}+03$ \\
\hline $1.01 \mathrm{E}+04$ & $6.44 \mathrm{E}+02$ & $9.49 \mathrm{E}+02$ & $1.29 \mathrm{E}+03$ & $7.73 \mathrm{E}+02$ & $1.43 \mathrm{E}+03$ \\
\hline $1.24 \mathrm{E}+04$ & $5.80 \mathrm{E}+02$ & $8.54 \mathrm{E}+02$ & $1.15 \mathrm{E}+03$ & $7.44 \mathrm{E}+02$ & $1.32 \mathrm{E}+03$ \\
\hline $1.53 \mathrm{E}+04$ & $5.11 \mathrm{E}+02$ & $7.54 \mathrm{E}+02$ & $1.01 \mathrm{E}+03$ & $7.10 \mathrm{E}+02$ & $1.21 \mathrm{E}+03$ \\
\hline $1.88 \mathrm{E}+04$ & $4.42 \mathrm{E}+02$ & $6.53 \mathrm{E}+02$ & $8.74 \mathrm{E}+02$ & $6.71 \mathrm{E}+02$ & $1.10 \mathrm{E}+03$ \\
\hline $2.30 \mathrm{E}+04$ & $3.74 \mathrm{E}+02$ & $5.55 \mathrm{E}+02$ & $7.39 \mathrm{E}+02$ & $6.29 \mathrm{E}+02$ & $9.83 \mathrm{E}+02$ \\
\hline $2.83 \mathrm{E}+04$ & $3.10 \mathrm{E}+02$ & $4.63 \mathrm{E}+02$ & $6.15 \mathrm{E}+02$ & $5.85 \mathrm{E}+02$ & $8.78 \mathrm{E}+02$ \\
\hline $3.47 \mathrm{E}+04$ & $2.54 \mathrm{E}+02$ & $3.82 \mathrm{E}+02$ & $5.05 \mathrm{E}+02$ & $5.42 \mathrm{E}+02$ & $7.83 \mathrm{E}+02$ \\
\hline $3.65 \mathrm{E}+04$ & $2.42 \mathrm{E}+02$ & $3.63 \mathrm{E}+02$ & $4.81 \mathrm{E}+02$ & $5.32 \mathrm{E}+02$ & $7.61 \mathrm{E}+02$ \\
\hline
\end{tabular}




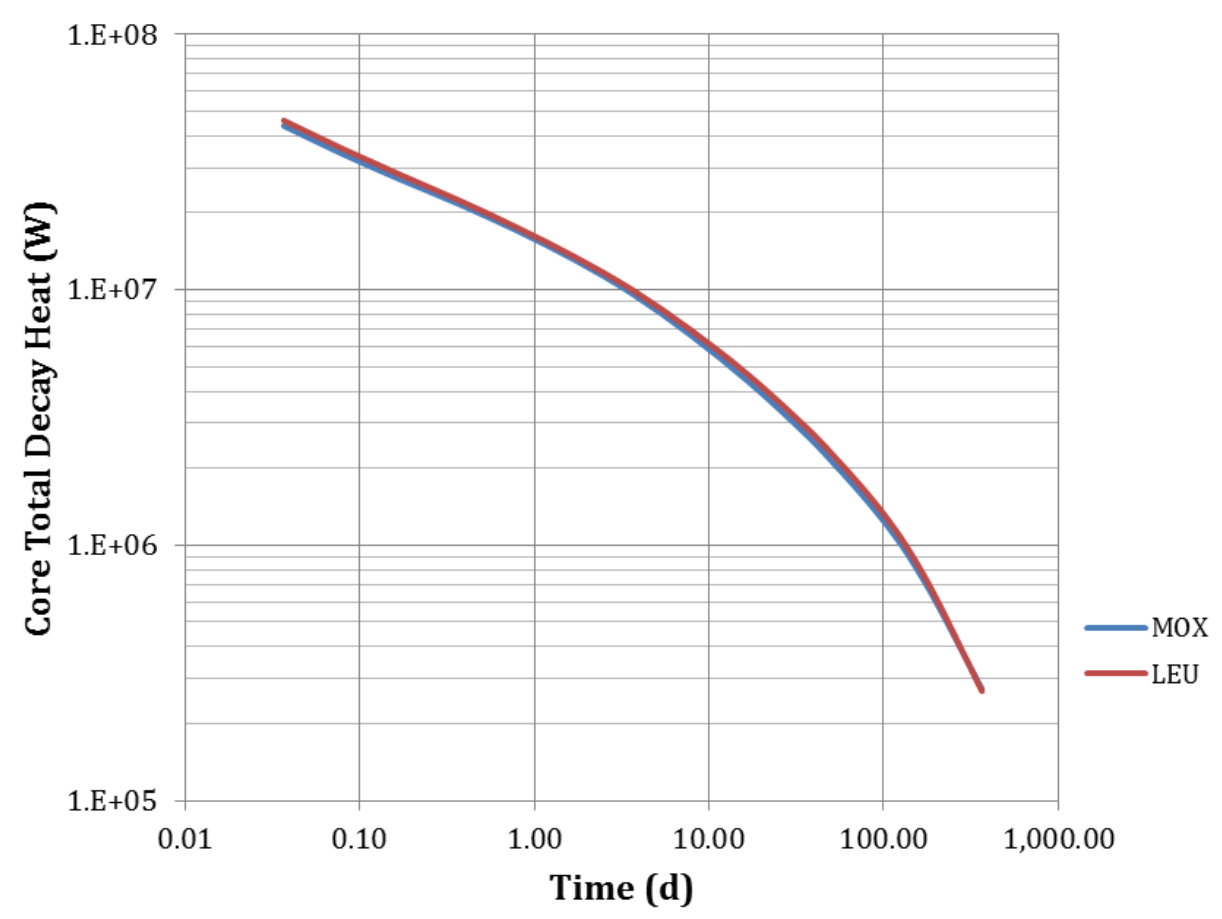

Figure 20. Comparison of decay heat in SQN equilibrium MOX and LEU cores - 1 year.

Table 15. Decay heat for the SQN LEU and MOX cores - 1 year

\begin{tabular}{||c|c|c||c|c|c||}
\hline Time (d) & LEU $(\mathbf{W})$ & MOX $(W)$ & Time $(d)$ & LEU (W) & MOX (W) \\
\hline \hline $0.00 \mathrm{E}+00$ & $2.19 \mathrm{E}+08$ & $2.05 \mathrm{E}+08$ & $5.48 \mathrm{E}+01$ & $2.14 \mathrm{E}+06$ & $2.00 \mathrm{E}+06$ \\
\hline $3.65 \mathrm{E}-03$ & $4.60 \mathrm{E}+07$ & $4.38 \mathrm{E}+07$ & $6.73 \mathrm{E}+01$ & $1.83 \mathrm{E}+06$ & $1.72 \mathrm{E}+06$ \\
\hline $1.02 \mathrm{E}-02$ & $3.58 \mathrm{E}+07$ & $3.41 \mathrm{E}+07$ & $8.26 \mathrm{E}+01$ & $1.56 \mathrm{E}+06$ & $1.46 \mathrm{E}+06$ \\
\hline $2.83 \mathrm{E}-02$ & $2.82 \mathrm{E}+07$ & $2.71 \mathrm{E}+07$ & $1.01 \mathrm{E}+02$ & $1.31 \mathrm{E}+06$ & $1.23 \mathrm{E}+06$ \\
\hline $7.87 \mathrm{E}-02$ & $2.25 \mathrm{E}+07$ & $2.17 \mathrm{E}+07$ & $1.24 \mathrm{E}+02$ & $1.08 \mathrm{E}+06$ & $1.02 \mathrm{E}+06$ \\
\hline $2.19 \mathrm{E}-01$ & $1.76 \mathrm{E}+07$ & $1.71 \mathrm{E}+07$ & $1.53 \mathrm{E}+02$ & $8.64 \mathrm{E}+05$ & $8.21 \mathrm{E}+05$ \\
\hline $6.09 \mathrm{E}-01$ & $1.35 \mathrm{E}+07$ & $1.31 \mathrm{E}+07$ & $1.88 \mathrm{E}+02$ & $6.73 \mathrm{E}+05$ & $6.47 \mathrm{E}+05$ \\
\hline $1.70 \mathrm{E}+00$ & $9.97 \mathrm{E}+06$ & $9.63 \mathrm{E}+06$ & $2.30 \mathrm{E}+02$ & $5.12 \mathrm{E}+05$ & $5.00 \mathrm{E}+05$ \\
\hline $4.72 \mathrm{E}+00$ & $6.95 \mathrm{E}+06$ & $6.63 \mathrm{E}+06$ & $2.83 \mathrm{E}+02$ & $3.84 \mathrm{E}+05$ & $3.82 \mathrm{E}+05$ \\
\hline $1.31 \mathrm{E}+01$ & $4.61 \mathrm{E}+06$ & $4.35 \mathrm{E}+06$ & $3.47 \mathrm{E}+02$ & $2.88 \mathrm{E}+05$ & $2.92 \mathrm{E}+05$ \\
\hline $3.65 \mathrm{E}+01$ & $2.86 \mathrm{E}+06$ & $2.68 \mathrm{E}+06$ & $3.65 \mathrm{E}+02$ & $2.68 \mathrm{E}+05$ & $2.73 \mathrm{E}+05$ \\
\hline \hline \multicolumn{7}{|l}{}
\end{tabular}




\section{BFN}

The total EOC decay heat trajectories were calculated for each burnup group of each of the BFN MOX and LEU assembly types. This was accomplished by taking the fuel composition at the burnup step closest to the average burnup of each group and simulating a 100-year decay period. The group-average burnups were not exactly the same as those of a particular burnup step, and the next higher burnup value was chosen as the starting point for the decays. Figure 21 presents a comparison of the individual decay heat trajectories, in W/MTHM, of the "A" fuel assemblies in the BFN equilibrium LEU core over a 100 -year period (expressed in days) following discharge from the core. The numerical values are summarized in Table 16. These data are presented for BFN LEU core assemblies " $B$ " and " $C$ " in Figure 22, Table 17 and Figure 23, Table 18, respectively.

Similar data for the BFN equilibrium MOX core LEU fuel assemblies "A" and "B" are presented in Figure 24, Table 19 and Figure 25, Table 20; for MOX fuel assemblies " $\mathrm{C}$ " and "D," data are presented in Figure 26, Table 21 and Figure 27, Table 22, respectively.

The total EOC decay heat trajectories, in watts, for the BFN MOX and LEU cores were obtained by creating new TRITON ft71001 nuclide concentration files for the MOX and LEU cores using the core fractions shown in Table 3 and Table 6, respectively. The new ft71001 files contain the nuclide concentrations of each assembly type, at the burnup step with the burnup closest to the average burnup of a burnup class weighted by their core fractions. The total core average decay heat as a function of time was then calculated by ORIGEN using the core-average set of nuclide concentrations in the new ft71001 files.

Figure 28 presents the core total decay heat curves decayed for a period of 1 year. Since the burnup steps did not, in general, occur at the average burnup of the burnup classes, the next step of burnup greater than the class average was chosen for inclusion in the $\mathrm{ft} 71001$ files. The decay heat data are reproduced in tabular form in Table 23. 


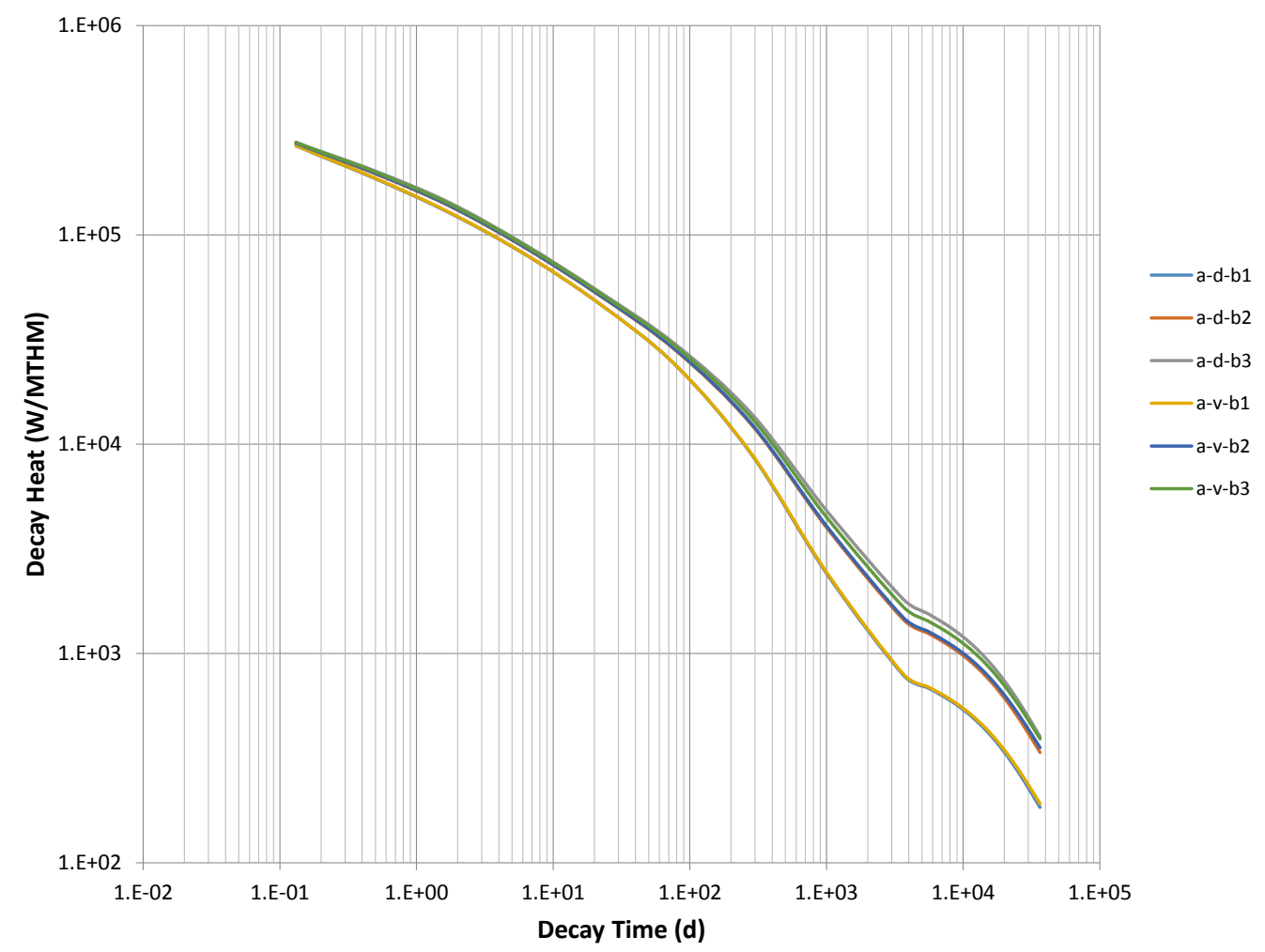

Figure 21. Comparison of " $A$ " assembly decay heat trajectories in the BFN equilibrium LEU core - 100 years.

Table 16. Decay heat (W/MTHM) for the fuel "A" assembly in the BFN LEU core

\begin{tabular}{||c|c|c|c|c|c|c||}
\hline \multirow{2}{*}{ Time (d) } & \multicolumn{3}{|c|}{ Dominant zone } & \multicolumn{3}{c||}{ Vanished zone } \\
\cline { 2 - 7 } & $\mathbf{b 1}$ & $\mathbf{b 2}$ & $\mathbf{b 3}$ & $\mathbf{b 1}$ & $\mathbf{b 2}$ & b3 \\
\hline \hline $0.00 \mathrm{E}+00$ & $1.80 \mathrm{E}+06$ & $1.76 \mathrm{E}+06$ & $1.75 \mathrm{E}+06$ & $1.81 \mathrm{E}+06$ & $1.76 \mathrm{E}+06$ & $1.76 \mathrm{E}+06$ \\
\hline $3.65 \mathrm{E}-02$ & $3.93 \mathrm{E}+05$ & $3.94 \mathrm{E}+05$ & $3.97 \mathrm{E}+05$ & $3.94 \mathrm{E}+05$ & $3.96 \mathrm{E}+05$ & $3.97 \mathrm{E}+05$ \\
\hline $1.31 \mathrm{E}-01$ & $2.67 \mathrm{E}+05$ & $2.73 \mathrm{E}+05$ & $2.77 \mathrm{E}+05$ & $2.68 \mathrm{E}+05$ & $2.74 \mathrm{E}+05$ & $2.76 \mathrm{E}+05$ \\
\hline $4.72 \mathrm{E}-01$ & $1.89 \mathrm{E}+05$ & $1.99 \mathrm{E}+05$ & $2.05 \mathrm{E}+05$ & $1.90 \mathrm{E}+05$ & $2.00 \mathrm{E}+05$ & $2.03 \mathrm{E}+05$ \\
\hline $1.70 \mathrm{E}+00$ & $1.29 \mathrm{E}+05$ & $1.39 \mathrm{E}+05$ & $1.44 \mathrm{E}+05$ & $1.29 \mathrm{E}+05$ & $1.39 \mathrm{E}+05$ & $1.42 \mathrm{E}+05$ \\
\hline $6.09 \mathrm{E}+00$ & $8.14 \mathrm{E}+04$ & $8.75 \mathrm{E}+04$ & $9.06 \mathrm{E}+04$ & $8.16 \mathrm{E}+04$ & $8.79 \mathrm{E}+04$ & $8.95 \mathrm{E}+04$ \\
\hline $2.19 \mathrm{E}+01$ & $4.68 \mathrm{E}+04$ & $5.13 \mathrm{E}+04$ & $5.34 \mathrm{E}+04$ & $4.69 \mathrm{E}+04$ & $5.15 \mathrm{E}+04$ & $5.26 \mathrm{E}+04$ \\
\hline $7.87 \mathrm{E}+01$ & $2.38 \mathrm{E}+04$ & $2.81 \mathrm{E}+04$ & $3.00 \mathrm{E}+04$ & $2.39 \mathrm{E}+04$ & $2.83 \mathrm{E}+04$ & $2.93 \mathrm{E}+04$ \\
\hline $2.83 \mathrm{E}+02$ & $8.94 \mathrm{E}+03$ & $1.24 \mathrm{E}+04$ & $1.40 \mathrm{E}+04$ & $9.00 \mathrm{E}+03$ & $1.25 \mathrm{E}+04$ & $1.33 \mathrm{E}+04$ \\
\hline $1.02 \mathrm{E}+03$ & $2.38 \mathrm{E}+03$ & $3.95 \mathrm{E}+03$ & $4.76 \mathrm{E}+03$ & $2.41 \mathrm{E}+03$ & $4.02 \mathrm{E}+03$ & $4.42 \mathrm{E}+03$ \\
\hline $3.65 \mathrm{E}+03$ & $7.88 \mathrm{E}+02$ & $1.45 \mathrm{E}+03$ & $1.81 \mathrm{E}+03$ & $7.96 \mathrm{E}+02$ & $1.49 \mathrm{E}+03$ & $1.67 \mathrm{E}+03$ \\
\hline $5.48 \mathrm{E}+03$ & $6.87 \mathrm{E}+02$ & $1.25 \mathrm{E}+03$ & $1.55 \mathrm{E}+03$ & $6.94 \mathrm{E}+02$ & $1.28 \mathrm{E}+03$ & $1.43 \mathrm{E}+03$ \\
\hline $6.73 \mathrm{E}+03$ & $6.39 \mathrm{E}+02$ & $1.16 \mathrm{E}+03$ & $1.44 \mathrm{E}+03$ & $6.47 \mathrm{E}+02$ & $1.19 \mathrm{E}+03$ & $1.33 \mathrm{E}+03$ \\
\hline $8.26 \mathrm{E}+03$ & $5.89 \mathrm{E}+02$ & $1.07 \mathrm{E}+03$ & $1.32 \mathrm{E}+03$ & $5.97 \mathrm{E}+02$ & $1.10 \mathrm{E}+03$ & $1.22 \mathrm{E}+03$ \\
\hline $1.01 \mathrm{E}+04$ & $5.36 \mathrm{E}+02$ & $9.71 \mathrm{E}+02$ & $1.19 \mathrm{E}+03$ & $5.43 \mathrm{E}+02$ & $9.96 \mathrm{E}+02$ & $1.11 \mathrm{E}+03$ \\
\hline $1.24 \mathrm{E}+04$ & $4.78 \mathrm{E}+02$ & $8.66 \mathrm{E}+02$ & $1.06 \mathrm{E}+03$ & $4.86 \mathrm{E}+02$ & $8.90 \mathrm{E}+02$ & $9.89 \mathrm{E}+02$ \\
\hline $1.53 \mathrm{E}+04$ & $4.18 \mathrm{E}+02$ & $7.57 \mathrm{E}+02$ & $9.21 \mathrm{E}+02$ & $4.26 \mathrm{E}+02$ & $7.80 \mathrm{E}+02$ & $8.65 \mathrm{E}+02$ \\
\hline $1.88 \mathrm{E}+04$ & $3.57 \mathrm{E}+02$ & $6.47 \mathrm{E}+02$ & $7.83 \mathrm{E}+02$ & $3.65 \mathrm{E}+02$ & $6.69 \mathrm{E}+02$ & $7.41 \mathrm{E}+02$ \\
\hline $2.30 \mathrm{E}+04$ & $2.98 \mathrm{E}+02$ & $5.41 \mathrm{E}+02$ & $6.51 \mathrm{E}+02$ & $3.05 \mathrm{E}+02$ & $5.61 \mathrm{E}+02$ & $6.20 \mathrm{E}+02$ \\
\hline $2.83 \mathrm{E}+04$ & $2.43 \mathrm{E}+02$ & $4.42 \mathrm{E}+02$ & $5.30 \mathrm{E}+02$ & $2.50 \mathrm{E}+02$ & $4.62 \mathrm{E}+02$ & $5.09 \mathrm{E}+02$ \\
\hline $3.47 \mathrm{E}+04$ & $1.95 \mathrm{E}+02$ & $3.56 \mathrm{E}+02$ & $4.24 \mathrm{E}+02$ & $2.02 \mathrm{E}+02$ & $3.75 \mathrm{E}+02$ & $4.12 \mathrm{E}+02$ \\
\hline $3.65 \mathrm{E}+04$ & $1.84 \mathrm{E}+02$ & $3.37 \mathrm{E}+02$ & $4.01 \mathrm{E}+02$ & $1.91 \mathrm{E}+02$ & $3.55 \mathrm{E}+02$ & $3.91 \mathrm{E}+02$ \\
\hline \hline
\end{tabular}




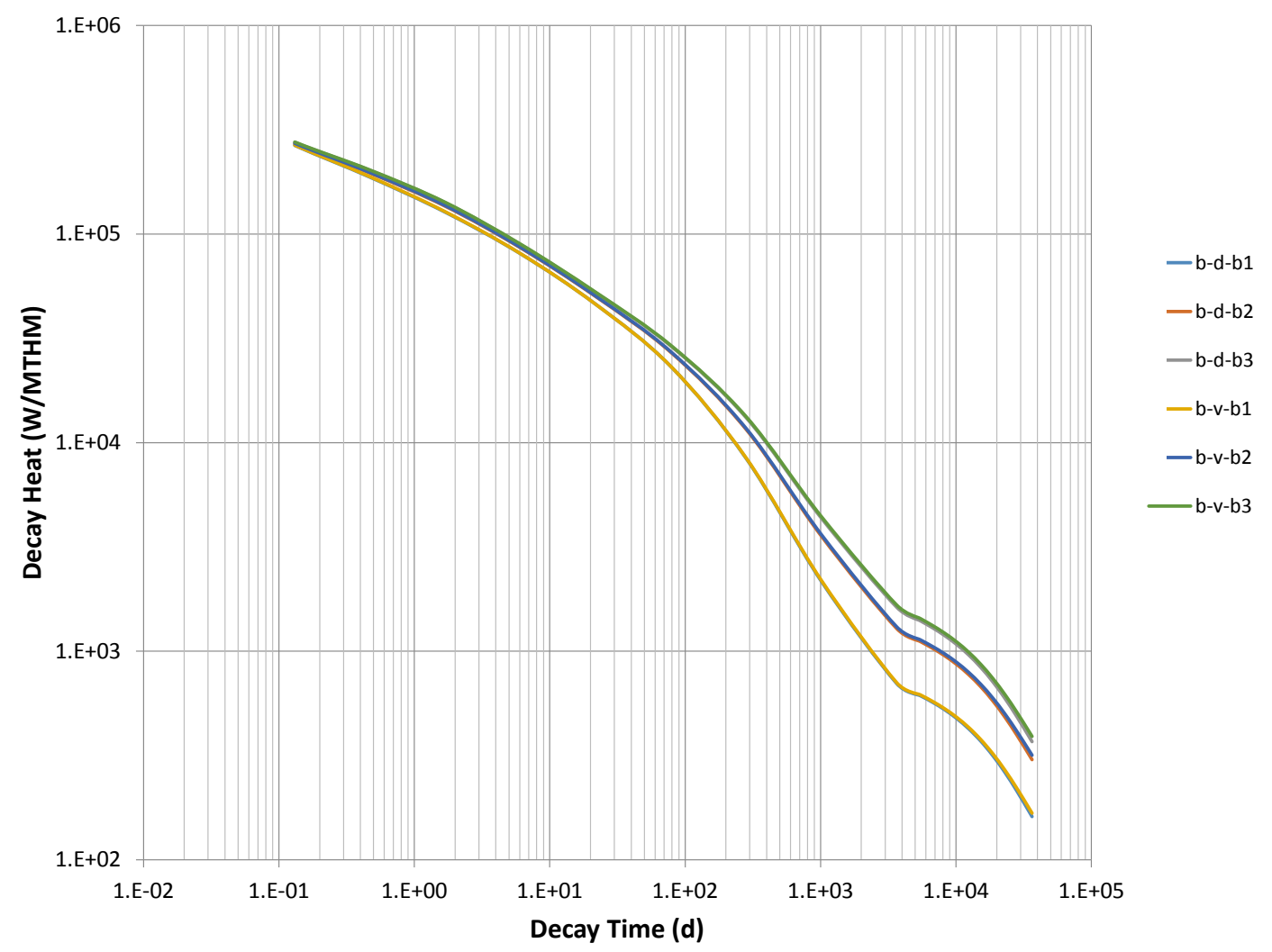

Figure 22. Comparison of "B" assembly decay heat trajectories in the BFN equilibrium LEU core - 100 years.

Table 17. Decay heat (W/MTHM) for the fuel "B" assembly in the BFN LEU core

\begin{tabular}{||c||c|c|c|c|c|c||}
\hline \multirow{2}{*}{ Time (d) } & \multicolumn{3}{|c|}{ Dominant zone } & \multicolumn{3}{c||}{ Vanished zone } \\
\cline { 2 - 7 } & b1 & b2 & b3 & b1 & b2 & b3 \\
\hline \hline $0.00 \mathrm{E}+00$ & $1.81 \mathrm{E}+06$ & $1.76 \mathrm{E}+06$ & $1.75 \mathrm{E}+06$ & $1.81 \mathrm{E}+06$ & $1.77 \mathrm{E}+06$ & $1.76 \mathrm{E}+06$ \\
\hline $3.65 \mathrm{E}-02$ & $3.93 \mathrm{E}+05$ & $3.93 \mathrm{E}+05$ & $3.96 \mathrm{E}+05$ & $3.94 \mathrm{E}+05$ & $3.95 \mathrm{E}+05$ & $3.97 \mathrm{E}+05$ \\
\hline $1.31 \mathrm{E}-01$ & $2.66 \mathrm{E}+05$ & $2.71 \mathrm{E}+05$ & $2.75 \mathrm{E}+05$ & $2.67 \mathrm{E}+05$ & $2.72 \mathrm{E}+05$ & $2.76 \mathrm{E}+05$ \\
\hline $4.72 \mathrm{E}-01$ & $1.87 \mathrm{E}+05$ & $1.96 \mathrm{E}+05$ & $2.02 \mathrm{E}+05$ & $1.88 \mathrm{E}+05$ & $1.97 \mathrm{E}+05$ & $2.03 \mathrm{E}+05$ \\
\hline $1.70 \mathrm{E}+00$ & $1.27 \mathrm{E}+05$ & $1.36 \mathrm{E}+05$ & $1.41 \mathrm{E}+05$ & $1.28 \mathrm{E}+05$ & $1.37 \mathrm{E}+05$ & $1.42 \mathrm{E}+05$ \\
\hline $6.09 \mathrm{E}+00$ & $8.05 \mathrm{E}+04$ & $8.60 \mathrm{E}+04$ & $8.91 \mathrm{E}+04$ & $8.07 \mathrm{E}+04$ & $8.64 \mathrm{E}+04$ & $8.95 \mathrm{E}+04$ \\
\hline $2.19 \mathrm{E}+01$ & $4.61 \mathrm{E}+04$ & $5.02 \mathrm{E}+04$ & $5.23 \mathrm{E}+04$ & $4.61 \mathrm{E}+04$ & $5.04 \mathrm{E}+04$ & $5.26 \mathrm{E}+04$ \\
\hline $7.87 \mathrm{E}+01$ & $2.32 \mathrm{E}+04$ & $2.71 \mathrm{E}+04$ & $2.90 \mathrm{E}+04$ & $2.32 \mathrm{E}+04$ & $2.73 \mathrm{E}+04$ & $2.92 \mathrm{E}+04$ \\
\hline $2.83 \mathrm{E}+02$ & $8.41 \mathrm{E}+03$ & $1.16 \mathrm{E}+04$ & $1.32 \mathrm{E}+04$ & $8.45 \mathrm{E}+03$ & $1.17 \mathrm{E}+04$ & $1.33 \mathrm{E}+04$ \\
\hline $1.02 \mathrm{E}+03$ & $2.16 \mathrm{E}+03$ & $3.57 \mathrm{E}+03$ & $4.35 \mathrm{E}+03$ & $2.19 \mathrm{E}+03$ & $3.63 \mathrm{E}+03$ & $4.42 \mathrm{E}+03$ \\
\hline $3.65 \mathrm{E}+03$ & $7.02 \mathrm{E}+02$ & $1.29 \mathrm{E}+03$ & $1.63 \mathrm{E}+03$ & $7.09 \mathrm{E}+02$ & $1.31 \mathrm{E}+03$ & $1.67 \mathrm{E}+03$ \\
\hline $5.48 \mathrm{E}+03$ & $6.13 \mathrm{E}+02$ & $1.11 \mathrm{E}+03$ & $1.40 \mathrm{E}+03$ & $6.19 \mathrm{E}+02$ & $1.14 \mathrm{E}+03$ & $1.43 \mathrm{E}+03$ \\
\hline $6.73 \mathrm{E}+03$ & $5.70 \mathrm{E}+02$ & $1.04 \mathrm{E}+03$ & $1.30 \mathrm{E}+03$ & $5.76 \mathrm{E}+02$ & $1.06 \mathrm{E}+03$ & $1.33 \mathrm{E}+03$ \\
\hline $8.26 \mathrm{E}+03$ & $5.25 \mathrm{E}+02$ & $9.53 \mathrm{E}+02$ & $1.19 \mathrm{E}+03$ & $5.31 \mathrm{E}+02$ & $9.73 \mathrm{E}+02$ & $1.22 \mathrm{E}+03$ \\
\hline $1.01 \mathrm{E}+04$ & $4.77 \mathrm{E}+02$ & $8.66 \mathrm{E}+02$ & $1.08 \mathrm{E}+03$ & $4.83 \mathrm{E}+02$ & $8.86 \mathrm{E}+02$ & $1.11 \mathrm{E}+03$ \\
\hline $1.24 \mathrm{E}+04$ & $4.25 \mathrm{E}+02$ & $7.73 \mathrm{E}+02$ & $9.61 \mathrm{E}+02$ & $4.32 \mathrm{E}+02$ & $7.92 \mathrm{E}+02$ & $9.89 \mathrm{E}+02$ \\
\hline $1.53 \mathrm{E}+04$ & $3.71 \mathrm{E}+02$ & $6.77 \mathrm{E}+02$ & $8.38 \mathrm{E}+02$ & $3.78 \mathrm{E}+02$ & $6.95 \mathrm{E}+02$ & $8.65 \mathrm{E}+02$ \\
\hline $1.88 \mathrm{E}+04$ & $3.16 \mathrm{E}+02$ & $5.79 \mathrm{E}+02$ & $7.15 \mathrm{E}+02$ & $3.23 \mathrm{E}+02$ & $5.97 \mathrm{E}+02$ & $7.40 \mathrm{E}+02$ \\
\hline $2.30 \mathrm{E}+04$ & $2.63 \mathrm{E}+02$ & $4.84 \mathrm{E}+02$ & $5.96 \mathrm{E}+02$ & $2.70 \mathrm{E}+02$ & $5.01 \mathrm{E}+02$ & $6.20 \mathrm{E}+02$ \\
\hline $2.83 \mathrm{E}+04$ & $2.14 \mathrm{E}+02$ & $3.96 \mathrm{E}+02$ & $4.86 \mathrm{E}+02$ & $2.20 \mathrm{E}+02$ & $4.13 \mathrm{E}+02$ & $5.09 \mathrm{E}+02$ \\
\hline $3.47 \mathrm{E}+04$ & $1.71 \mathrm{E}+02$ & $3.20 \mathrm{E}+02$ & $3.90 \mathrm{E}+02$ & $1.77 \mathrm{E}+02$ & $3.35 \mathrm{E}+02$ & $4.12 \mathrm{E}+02$ \\
\hline $3.65 \mathrm{E}+04$ & $1.61 \mathrm{E}+02$ & $3.03 \mathrm{E}+02$ & $3.69 \mathrm{E}+02$ & $1.68 \mathrm{E}+02$ & $3.18 \mathrm{E}+02$ & $3.90 \mathrm{E}+02$ \\
\hline \hline
\end{tabular}




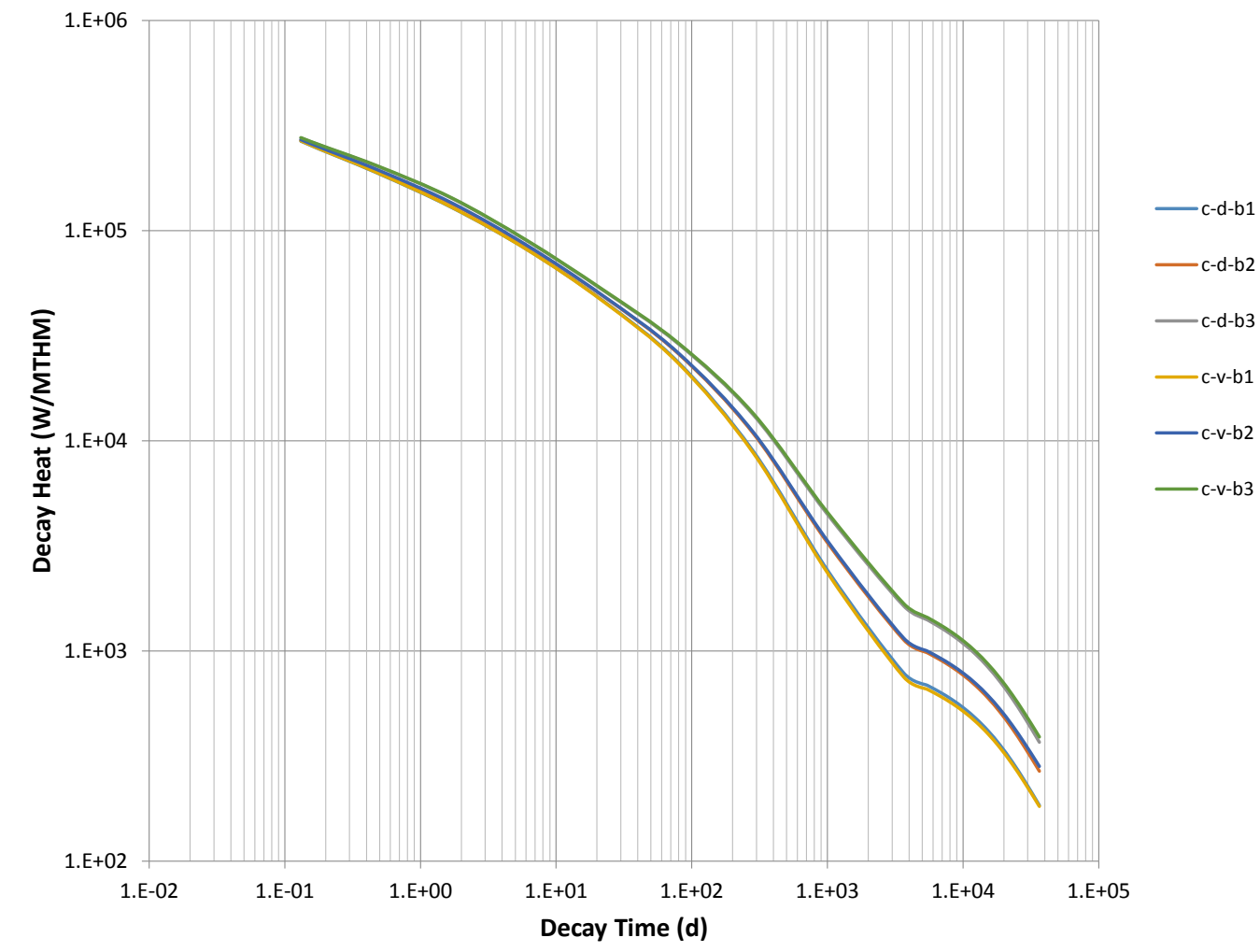

Figure 23. Comparison of "C" assembly decay heat trajectories in the BFN equilibrium LEU core - 100 years.

Table 18. Decay heat (W/MTHM) for the fuel " $C$ " assembly in the BFN LEU core

\begin{tabular}{||c||c|c|c|c|c|c||}
\hline \multirow{2}{*}{ Time (d) } & \multicolumn{3}{|c|}{ Dominant zone } & \multicolumn{3}{c||}{ Vanished zone } \\
\cline { 2 - 7 } & $\mathbf{b 1}$ & $\mathbf{b 2}$ & $\mathbf{b 3}$ & $\mathbf{b 1}$ & b2 & b3 \\
\hline \hline $0.00 \mathrm{E}+00$ & $1.80 \mathrm{E}+06$ & $1.77 \mathrm{E}+06$ & $1.75 \mathrm{E}+06$ & $1.80 \mathrm{E}+06$ & $1.77 \mathrm{E}+06$ & $1.75 \mathrm{E}+06$ \\
\hline $3.65 \mathrm{E}-02$ & $3.93 \mathrm{E}+05$ & $3.93 \mathrm{E}+05$ & $3.97 \mathrm{E}+05$ & $3.94 \mathrm{E}+05$ & $3.94 \mathrm{E}+05$ & $3.98 \mathrm{E}+05$ \\
\hline $1.31 \mathrm{E}-01$ & $2.67 \mathrm{E}+05$ & $2.70 \mathrm{E}+05$ & $2.76 \mathrm{E}+05$ & $2.68 \mathrm{E}+05$ & $2.71 \mathrm{E}+05$ & $2.77 \mathrm{E}+05$ \\
\hline $4.72 \mathrm{E}-01$ & $1.89 \mathrm{E}+05$ & $1.95 \mathrm{E}+05$ & $2.03 \mathrm{E}+05$ & $1.90 \mathrm{E}+05$ & $1.96 \mathrm{E}+05$ & $2.05 \mathrm{E}+05$ \\
\hline $1.70 \mathrm{E}+00$ & $1.29 \mathrm{E}+05$ & $1.35 \mathrm{E}+05$ & $1.43 \mathrm{E}+05$ & $1.29 \mathrm{E}+05$ & $1.36 \mathrm{E}+05$ & $1.44 \mathrm{E}+05$ \\
\hline $6.09 \mathrm{E}+00$ & $8.15 \mathrm{E}+04$ & $8.47 \mathrm{E}+04$ & $8.94 \mathrm{E}+04$ & $8.13 \mathrm{E}+04$ & $8.52 \mathrm{E}+04$ & $9.00 \mathrm{E}+04$ \\
\hline $2.19 \mathrm{E}+01$ & $4.67 \mathrm{E}+04$ & $4.91 \mathrm{E}+04$ & $5.23 \mathrm{E}+04$ & $4.64 \mathrm{E}+04$ & $4.93 \mathrm{E}+04$ & $5.27 \mathrm{E}+04$ \\
\hline $7.87 \mathrm{E}+01$ & $2.38 \mathrm{E}+04$ & $2.62 \mathrm{E}+04$ & $2.91 \mathrm{E}+04$ & $2.36 \mathrm{E}+04$ & $2.64 \mathrm{E}+04$ & $2.94 \mathrm{E}+04$ \\
\hline $2.83 \mathrm{E}+02$ & $8.98 \mathrm{E}+03$ & $1.09 \mathrm{E}+04$ & $1.33 \mathrm{E}+04$ & $8.80 \mathrm{E}+03$ & $1.10 \mathrm{E}+04$ & $1.36 \mathrm{E}+04$ \\
\hline $1.02 \mathrm{E}+03$ & $2.39 \mathrm{E}+03$ & $3.23 \mathrm{E}+03$ & $4.41 \mathrm{E}+03$ & $2.32 \mathrm{E}+03$ & $3.30 \mathrm{E}+03$ & $4.51 \mathrm{E}+03$ \\
\hline $3.65 \mathrm{E}+03$ & $7.87 \mathrm{E}+02$ & $1.13 \mathrm{E}+03$ & $1.64 \mathrm{E}+03$ & $7.52 \mathrm{E}+02$ & $1.15 \mathrm{E}+03$ & $1.68 \mathrm{E}+03$ \\
\hline $5.48 \mathrm{E}+03$ & $6.86 \mathrm{E}+02$ & $9.80 \mathrm{E}+02$ & $1.41 \mathrm{E}+03$ & $6.56 \mathrm{E}+02$ & $9.98 \mathrm{E}+02$ & $1.44 \mathrm{E}+03$ \\
\hline $6.73 \mathrm{E}+03$ & $6.39 \mathrm{E}+02$ & $9.12 \mathrm{E}+02$ & $1.30 \mathrm{E}+03$ & $6.11 \mathrm{E}+02$ & $9.29 \mathrm{E}+02$ & $1.34 \mathrm{E}+03$ \\
\hline $8.26 \mathrm{E}+03$ & $5.89 \mathrm{E}+02$ & $8.40 \mathrm{E}+02$ & $1.19 \mathrm{E}+03$ & $5.64 \mathrm{E}+02$ & $8.57 \mathrm{E}+02$ & $1.23 \mathrm{E}+03$ \\
\hline $1.01 \mathrm{E}+04$ & $5.35 \mathrm{E}+02$ & $7.64 \mathrm{E}+02$ & $1.08 \mathrm{E}+03$ & $5.14 \mathrm{E}+02$ & $7.80 \mathrm{E}+02$ & $1.11 \mathrm{E}+03$ \\
\hline $1.24 \mathrm{E}+04$ & $4.78 \mathrm{E}+02$ & $6.83 \mathrm{E}+02$ & $9.62 \mathrm{E}+02$ & $4.60 \mathrm{E}+02$ & $6.99 \mathrm{E}+02$ & $9.92 \mathrm{E}+02$ \\
\hline $1.53 \mathrm{E}+04$ & $4.18 \mathrm{E}+02$ & $5.98 \mathrm{E}+02$ & $8.38 \mathrm{E}+02$ & $4.03 \mathrm{E}+02$ & $6.14 \mathrm{E}+02$ & $8.67 \mathrm{E}+02$ \\
\hline $1.88 \mathrm{E}+04$ & $3.57 \mathrm{E}+02$ & $5.12 \mathrm{E}+02$ & $7.15 \mathrm{E}+02$ & $3.46 \mathrm{E}+02$ & $5.28 \mathrm{E}+02$ & $7.41 \mathrm{E}+02$ \\
\hline $2.30 \mathrm{E}+04$ & $2.98 \mathrm{E}+02$ & $4.28 \mathrm{E}+02$ & $5.95 \mathrm{E}+02$ & $2.90 \mathrm{E}+02$ & $4.44 \mathrm{E}+02$ & $6.20 \mathrm{E}+02$ \\
\hline $2.83 \mathrm{E}+04$ & $2.43 \mathrm{E}+02$ & $3.51 \mathrm{E}+02$ & $4.85 \mathrm{E}+02$ & $2.38 \mathrm{E}+02$ & $3.66 \mathrm{E}+02$ & $5.09 \mathrm{E}+02$ \\
\hline $3.47 \mathrm{E}+04$ & $1.95 \mathrm{E}+02$ & $2.83 \mathrm{E}+02$ & $3.90 \mathrm{E}+02$ & $1.92 \mathrm{E}+02$ & $2.98 \mathrm{E}+02$ & $4.12 \mathrm{E}+02$ \\
\hline $3.65 \mathrm{E}+04$ & $1.84 \mathrm{E}+02$ & $2.68 \mathrm{E}+02$ & $3.69 \mathrm{E}+02$ & $1.82 \mathrm{E}+02$ & $2.83 \mathrm{E}+02$ & $3.90 \mathrm{E}+02$ \\
\hline
\end{tabular}




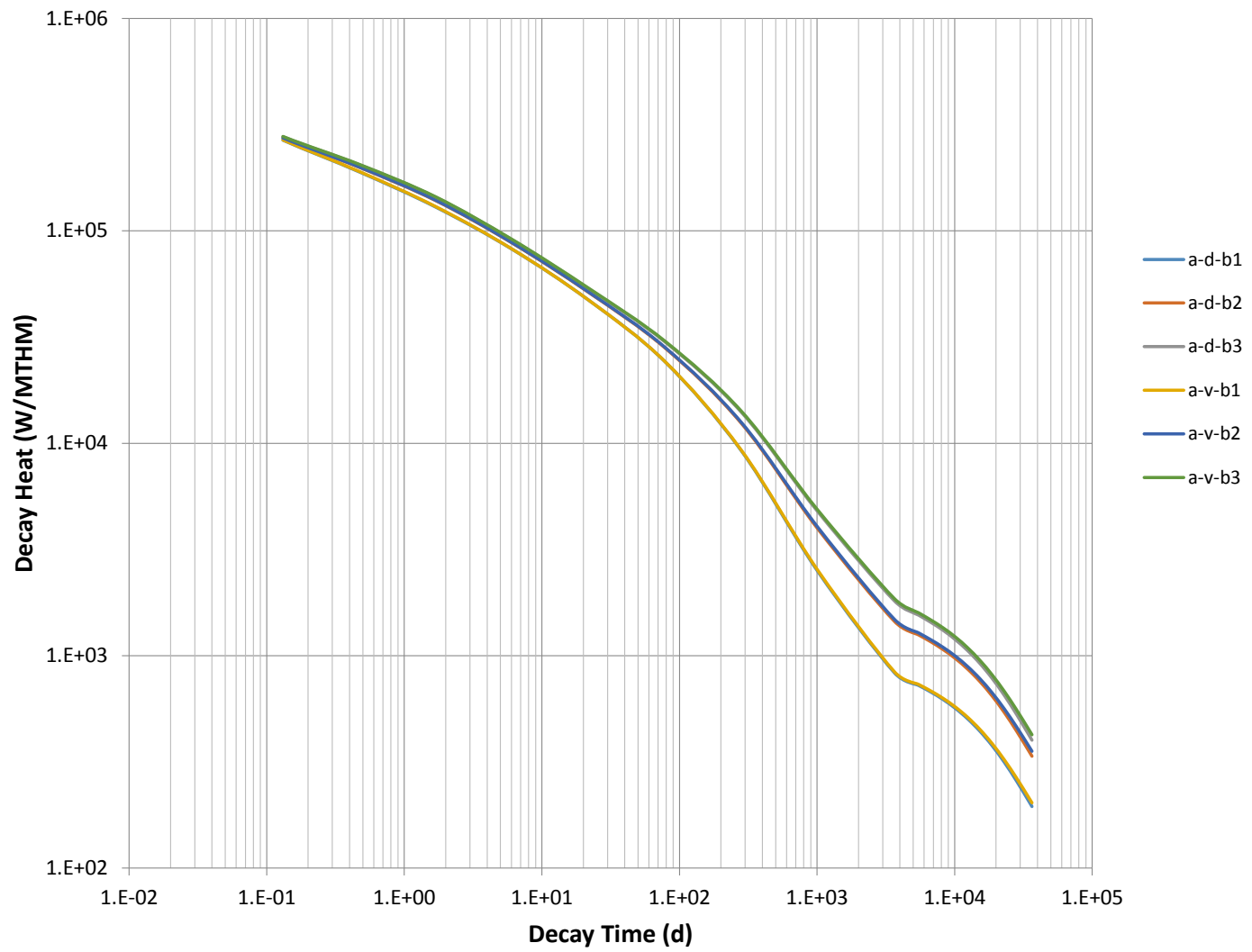

Figure 24. Comparison of " $A$ " assembly decay heat trajectories in the BFN equilibrium MOX core - 100 years.

Table 19. Decay heat (W/MTHM) for the fuel "A" assembly in the BFN MOX core

\begin{tabular}{|c||c|c|c|c|c|c||}
\hline \multirow{2}{*}{ Time (d) } & \multicolumn{3}{|c|}{ Dominant zone } & \multicolumn{3}{c||}{ Vanished zone } \\
\cline { 2 - 7 } & $\mathbf{b 1}$ & $\mathbf{b 2}$ & $\mathbf{b 3}$ & $\mathbf{b 1}$ & $\mathbf{b 2}$ & b3 \\
\hline \hline $0.00 \mathrm{E}+00$ & $1.80 \mathrm{E}+06$ & $1.76 \mathrm{E}+06$ & $1.75 \mathrm{E}+06$ & $1.80 \mathrm{E}+06$ & $1.76 \mathrm{E}+06$ & $1.76 \mathrm{E}+06$ \\
\hline $3.65 \mathrm{E}-02$ & $3.93 \mathrm{E}+05$ & $3.94 \mathrm{E}+05$ & $3.97 \mathrm{E}+05$ & $3.94 \mathrm{E}+05$ & $3.96 \mathrm{E}+05$ & $3.99 \mathrm{E}+05$ \\
\hline $1.31 \mathrm{E}-01$ & $2.67 \mathrm{E}+05$ & $2.73 \mathrm{E}+05$ & $2.77 \mathrm{E}+05$ & $2.68 \mathrm{E}+05$ & $2.74 \mathrm{E}+05$ & $2.78 \mathrm{E}+05$ \\
\hline $4.72 \mathrm{E}-01$ & $1.89 \mathrm{E}+05$ & $1.99 \mathrm{E}+05$ & $2.05 \mathrm{E}+05$ & $1.90 \mathrm{E}+05$ & $2.00 \mathrm{E}+05$ & $2.06 \mathrm{E}+05$ \\
\hline $1.70 \mathrm{E}+00$ & $1.29 \mathrm{E}+05$ & $1.39 \mathrm{E}+05$ & $1.44 \mathrm{E}+05$ & $1.30 \mathrm{E}+05$ & $1.39 \mathrm{E}+05$ & $1.45 \mathrm{E}+05$ \\
\hline $6.09 \mathrm{E}+00$ & $8.18 \mathrm{E}+04$ & $8.75 \mathrm{E}+04$ & $9.06 \mathrm{E}+04$ & $8.21 \mathrm{E}+04$ & $8.79 \mathrm{E}+04$ & $9.11 \mathrm{E}+04$ \\
\hline $2.19 \mathrm{E}+01$ & $4.72 \mathrm{E}+04$ & $5.13 \mathrm{E}+04$ & $5.34 \mathrm{E}+04$ & $4.72 \mathrm{E}+04$ & $5.15 \mathrm{E}+04$ & $5.37 \mathrm{E}+04$ \\
\hline $7.87 \mathrm{E}+01$ & $2.42 \mathrm{E}+04$ & $2.81 \mathrm{E}+04$ & $3.00 \mathrm{E}+04$ & $2.42 \mathrm{E}+04$ & $2.83 \mathrm{E}+04$ & $3.02 \mathrm{E}+04$ \\
\hline $2.83 \mathrm{E}+02$ & $9.19 \mathrm{E}+03$ & $1.24 \mathrm{E}+04$ & $1.40 \mathrm{E}+04$ & $9.25 \mathrm{E}+03$ & $1.25 \mathrm{E}+04$ & $1.41 \mathrm{E}+04$ \\
\hline $1.02 \mathrm{E}+03$ & $2.49 \mathrm{E}+03$ & $3.95 \mathrm{E}+03$ & $4.76 \mathrm{E}+03$ & $2.52 \mathrm{E}+03$ & $4.02 \mathrm{E}+03$ & $4.84 \mathrm{E}+03$ \\
\hline $3.65 \mathrm{E}+03$ & $8.31 \mathrm{E}+02$ & $1.45 \mathrm{E}+03$ & $1.81 \mathrm{E}+03$ & $8.41 \mathrm{E}+02$ & $1.49 \mathrm{E}+03$ & $1.86 \mathrm{E}+03$ \\
\hline $5.48 \mathrm{E}+03$ & $7.24 \mathrm{E}+02$ & $1.25 \mathrm{E}+03$ & $1.55 \mathrm{E}+03$ & $7.33 \mathrm{E}+02$ & $1.28 \mathrm{E}+03$ & $1.59 \mathrm{E}+03$ \\
\hline $6.73 \mathrm{E}+03$ & $6.74 \mathrm{E}+02$ & $1.16 \mathrm{E}+03$ & $1.44 \mathrm{E}+03$ & $6.82 \mathrm{E}+02$ & $1.19 \mathrm{E}+03$ & $1.47 \mathrm{E}+03$ \\
\hline $8.26 \mathrm{E}+03$ & $6.21 \mathrm{E}+02$ & $1.07 \mathrm{E}+03$ & $1.32 \mathrm{E}+03$ & $6.30 \mathrm{E}+02$ & $1.10 \mathrm{E}+03$ & $1.35 \mathrm{E}+03$ \\
\hline $1.01 \mathrm{E}+04$ & $5.65 \mathrm{E}+02$ & $9.71 \mathrm{E}+02$ & $1.19 \mathrm{E}+03$ & $5.73 \mathrm{E}+02$ & $9.96 \mathrm{E}+02$ & $1.23 \mathrm{E}+03$ \\
\hline $1.24 \mathrm{E}+04$ & $5.05 \mathrm{E}+02$ & $8.66 \mathrm{E}+02$ & $1.06 \mathrm{E}+03$ & $5.13 \mathrm{E}+02$ & $8.90 \mathrm{E}+02$ & $1.09 \mathrm{E}+03$ \\
\hline $1.53 \mathrm{E}+04$ & $4.41 \mathrm{E}+02$ & $7.57 \mathrm{E}+02$ & $9.21 \mathrm{E}+02$ & $4.50 \mathrm{E}+02$ & $7.80 \mathrm{E}+02$ & $9.52 \mathrm{E}+02$ \\
\hline $1.88 \mathrm{E}+04$ & $3.77 \mathrm{E}+02$ & $6.47 \mathrm{E}+02$ & $7.83 \mathrm{E}+02$ & $3.86 \mathrm{E}+02$ & $6.69 \mathrm{E}+02$ & $8.13 \mathrm{E}+02$ \\
\hline $2.30 \mathrm{E}+04$ & $3.15 \mathrm{E}+02$ & $5.41 \mathrm{E}+02$ & $6.51 \mathrm{E}+02$ & $3.23 \mathrm{E}+02$ & $5.61 \mathrm{E}+02$ & $6.79 \mathrm{E}+02$ \\
\hline $2.83 \mathrm{E}+04$ & $2.57 \mathrm{E}+02$ & $4.42 \mathrm{E}+02$ & $5.30 \mathrm{E}+02$ & $2.65 \mathrm{E}+02$ & $4.62 \mathrm{E}+02$ & $5.56 \mathrm{E}+02$ \\
\hline $3.47 \mathrm{E}+04$ & $2.06 \mathrm{E}+02$ & $3.56 \mathrm{E}+02$ & $4.24 \mathrm{E}+02$ & $2.14 \mathrm{E}+02$ & $3.75 \mathrm{E}+02$ & $4.49 \mathrm{E}+02$ \\
\hline $3.65 \mathrm{E}+04$ & $1.95 \mathrm{E}+02$ & $3.37 \mathrm{E}+02$ & $4.01 \mathrm{E}+02$ & $2.03 \mathrm{E}+02$ & $3.55 \mathrm{E}+02$ & $4.25 \mathrm{E}+02$ \\
\hline
\end{tabular}




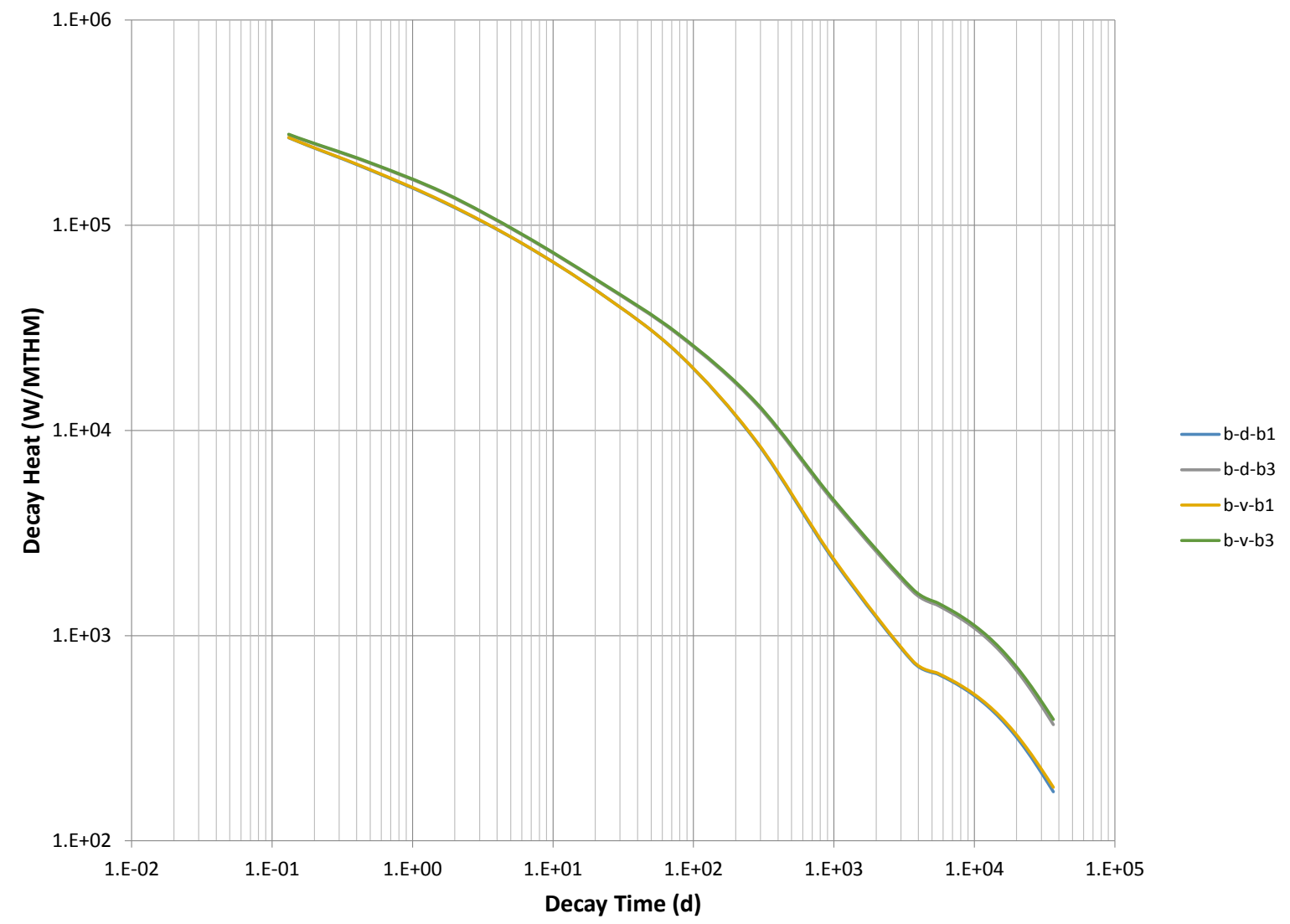

Figure 25. Comparison of "B" assembly decay heat trajectories in the BFN equilibrium MOX core - 100 years.

Table 20. Decay heat (W/MTHM) for the fuel "B" assembly in the BFN MOX core

\begin{tabular}{||c||c|c|c|c|c|c||}
\hline \hline \multirow{2}{*}{ Time (d) } & \multicolumn{2}{|c|}{ Dominant Zone } & \multicolumn{3}{c||}{ Vanished Zone } \\
\cline { 2 - 7 } & $\mathbf{b 1}$ & $\mathbf{b 2}$ & $\mathbf{b 3}$ & $\mathbf{b 1}$ & $\mathbf{b 2}$ & b3 \\
\hline \hline $0.00 \mathrm{E}+00$ & $1.80 \mathrm{E}+06$ & & $1.75 \mathrm{E}+06$ & $1.80 \mathrm{E}+06$ & & $1.75 \mathrm{E}+06$ \\
\hline $3.65 \mathrm{E}-02$ & $3.93 \mathrm{E}+05$ & & $3.97 \mathrm{E}+05$ & $3.94 \mathrm{E}+05$ & & $3.98 \mathrm{E}+05$ \\
\hline $1.31 \mathrm{E}-01$ & $2.67 \mathrm{E}+05$ & & $2.76 \mathrm{E}+05$ & $2.68 \mathrm{E}+05$ & & $2.77 \mathrm{E}+05$ \\
\hline $4.72 \mathrm{E}-01$ & $1.89 \mathrm{E}+05$ & & $2.03 \mathrm{E}+05$ & $1.90 \mathrm{E}+05$ & & $2.5 \mathrm{E}+05$ \\
\hline $1.70 \mathrm{E}+00$ & $1.29 \mathrm{E}+05$ & & $1.43 \mathrm{E}+05$ & $1.29 \mathrm{E}+05$ & & $1.44 \mathrm{E}+05$ \\
\hline $6.09 \mathrm{E}+00$ & $8.10 \mathrm{E}+04$ & & $8.94 \mathrm{E}+04$ & $8.13 \mathrm{E}+04$ & & $9.00 \mathrm{E}+04$ \\
\hline $2.19 \mathrm{E}+01$ & $4.64 \mathrm{E}+04$ & & $5.23 \mathrm{E}+04$ & $4.64 \mathrm{E}+04$ & & $5.27 \mathrm{E}+04$ \\
\hline $7.87 \mathrm{E}+01$ & $2.35 \mathrm{E}+04$ & & $2.91 \mathrm{E}+04$ & $2.36 \mathrm{E}+04$ & & $2.94 \mathrm{E}+04$ \\
\hline $2.83 \mathrm{E}+02$ & $8.71 \mathrm{E}+03$ & & $1.33 \mathrm{E}+04$ & $8.80 \mathrm{E}+03$ & & $1.36 \mathrm{E}+04$ \\
\hline $1.02 \mathrm{E}+03$ & $2.28 \mathrm{E}+03$ & & $4.41 \mathrm{E}+03$ & $2.32 \mathrm{E}+03$ & & $4.51 \mathrm{E}+03$ \\
\hline $3.65 \mathrm{E}+03$ & $7.44 \mathrm{E}+02$ & & $1.64 \mathrm{E}+03$ & $7.52 \mathrm{E}+02$ & & $1.68 \mathrm{E}+03$ \\
\hline $5.48 \mathrm{E}+03$ & $6.49 \mathrm{E}+02$ & & $1.41 \mathrm{E}+03$ & $6.56 \mathrm{E}+02$ & & $1.44 \mathrm{E}+03$ \\
\hline $6.73 \mathrm{E}+03$ & $6.04 \mathrm{E}+02$ & & $1.30 \mathrm{E}+03$ & $6.11 \mathrm{E}+02$ & & $1.34 \mathrm{E}+03$ \\
\hline $8.26 \mathrm{E}+03$ & $5.57 \mathrm{E}+02$ & & $1.19 \mathrm{E}+03$ & $5.64 \mathrm{E}+02$ & & $1.23 \mathrm{E}+03$ \\
\hline $1.01 \mathrm{E}+04$ & $5.06 \mathrm{E}+02$ & & $1.08 \mathrm{E}+03$ & $5.14 \mathrm{E}+02$ & & $1.11 \mathrm{E}+03$ \\
\hline $1.24 \mathrm{E}+04$ & $4.52 \mathrm{E}+02$ & & $9.62 \mathrm{E}+02$ & $4.60 \mathrm{E}+02$ & & $9.92 \mathrm{E}+02$ \\
\hline $1.53 \mathrm{E}+04$ & $3.95 \mathrm{E}+02$ & & $8.38 \mathrm{E}+02$ & $4.03 \mathrm{E}+02$ & & $8.67 \mathrm{E}+02$ \\
\hline $1.88 \mathrm{E}+04$ & $3.37 \mathrm{E}+02$ & & $7.15 \mathrm{E}+02$ & $3.46 \mathrm{E}+02$ & & $7.41 \mathrm{E}+02$ \\
\hline $2.30 \mathrm{E}+04$ & $2.81 \mathrm{E}+02$ & & $5.95 \mathrm{E}+02$ & $2.90 \mathrm{E}+02$ & & $6.20 \mathrm{E}+02$ \\
\hline $2.83 \mathrm{E}+04$ & $2.29 \mathrm{E}+02$ & & $4.85 \mathrm{E}+02$ & $2.38 \mathrm{E}+02$ & & $5.09 \mathrm{E}+02$ \\
\hline $3.47 \mathrm{E}+04$ & $1.83 \mathrm{E}+02$ & & $3.90 \mathrm{E}+02$ & $1.92 \mathrm{E}+02$ & & $4.12 \mathrm{E}+02$ \\
\hline $3.65 \mathrm{E}+04$ & $1.73 \mathrm{E}+02$ & & $3.69 \mathrm{E}+02$ & $1.82 \mathrm{E}+02$ & & $3.90 \mathrm{E}+02$ \\
\hline
\end{tabular}




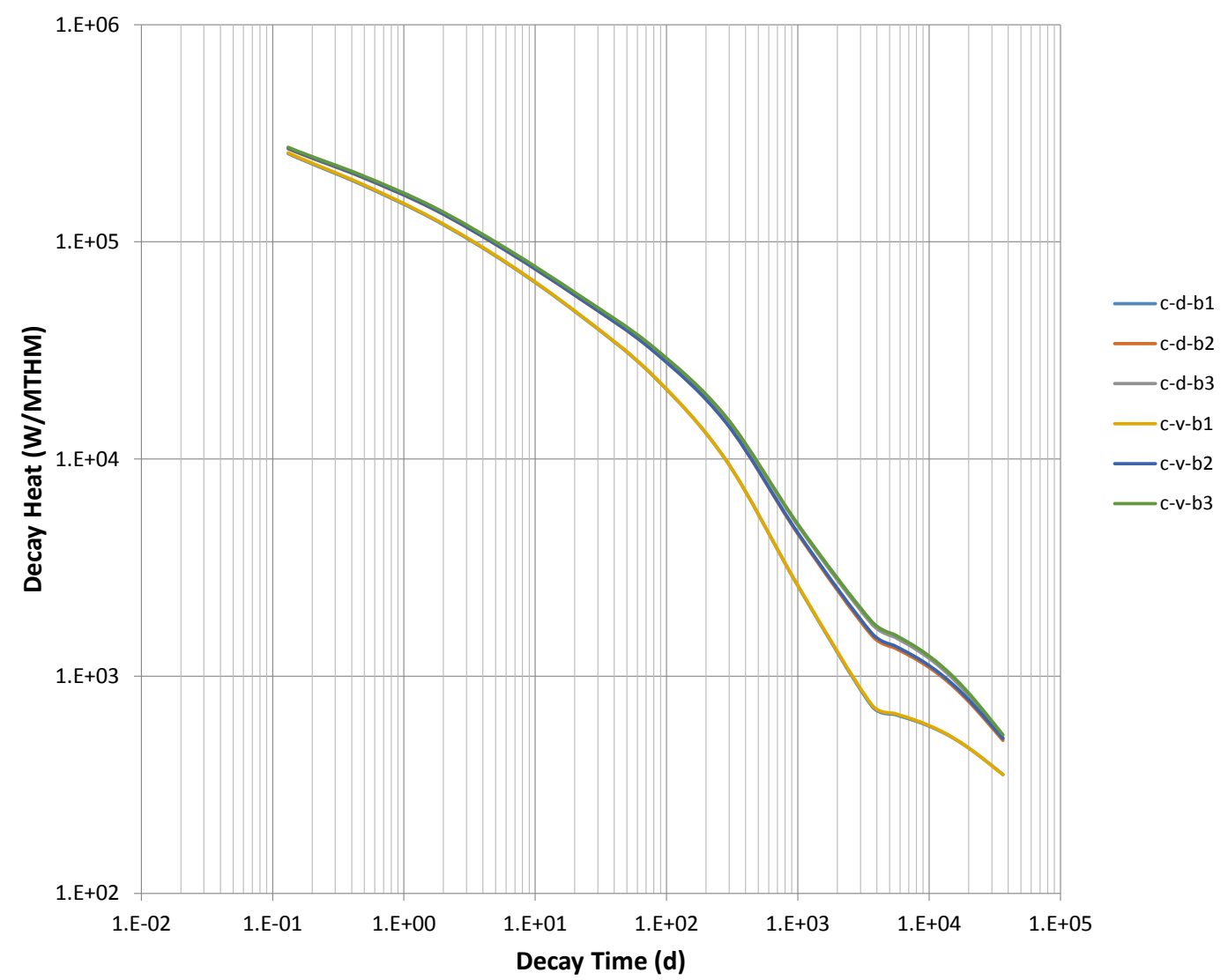

Figure 26. Comparison of " $C$ " assembly decay heat trajectories in the BFN equilibrium MOX core - 100 years.

Table 21. Decay heat (W/MTHM) for the fuel "C" assembly in the BFN MOX core

\begin{tabular}{||c|c|c|c|c|c|c||}
\hline \multirow{2}{*}{ Time (d) } & \multicolumn{3}{|c}{ Dominant zone } & \multicolumn{3}{c||}{ Vanished zone } \\
\cline { 2 - 7 } & b1 & b2 & b3 & b1 & b2 & b3 \\
\hline \hline $0.00 \mathrm{E}+00$ & $1.69 \mathrm{E}+06$ & $1.71 \mathrm{E}+06$ & $1.71 \mathrm{E}+06$ & $1.70 \mathrm{E}+06$ & $1.72 \mathrm{E}+06$ & $1.72 \mathrm{E}+06$ \\
\hline $3.65 \mathrm{E}-02$ & $3.73 \mathrm{E}+05$ & $3.84 \mathrm{E}+05$ & $3.87 \mathrm{E}+05$ & $3.75 \mathrm{E}+05$ & $3.86 \mathrm{E}+05$ & $3.89 \mathrm{E}+05$ \\
\hline $1.31 \mathrm{E}-01$ & $2.55 \mathrm{E}+05$ & $2.68 \mathrm{E}+05$ & $2.71 \mathrm{E}+05$ & $2.57 \mathrm{E}+05$ & $2.70 \mathrm{E}+05$ & $2.73 \mathrm{E}+05$ \\
\hline $4.72 \mathrm{E}-01$ & $1.84 \mathrm{E}+05$ & $1.99 \mathrm{E}+05$ & $2.02 \mathrm{E}+05$ & $1.85 \mathrm{E}+05$ & $2.00 \mathrm{E}+05$ & $2.03 \mathrm{E}+05$ \\
\hline $1.70 \mathrm{E}+00$ & $1.27 \mathrm{E}+05$ & $1.41 \mathrm{E}+05$ & $1.44 \mathrm{E}+05$ & $1.28 \mathrm{E}+05$ & $1.42 \mathrm{E}+05$ & $1.44 \mathrm{E}+05$ \\
\hline $6.09 \mathrm{E}+00$ & $7.96 \mathrm{E}+04$ & $9.03 \mathrm{E}+04$ & $9.23 \mathrm{E}+04$ & $8.01 \mathrm{E}+04$ & $9.08 \mathrm{E}+04$ & $9.27 \mathrm{E}+04$ \\
\hline $2.19 \mathrm{E}+01$ & $4.59 \mathrm{E}+04$ & $5.46 \mathrm{E}+04$ & $5.61 \mathrm{E}+04$ & $4.61 \mathrm{E}+04$ & $5.49 \mathrm{E}+04$ & $5.64 \mathrm{E}+04$ \\
\hline $7.87 \mathrm{E}+01$ & $2.42 \mathrm{E}+04$ & $3.15 \mathrm{E}+04$ & $3.27 \mathrm{E}+04$ & $2.43 \mathrm{E}+04$ & $3.16 \mathrm{E}+04$ & $3.29 \mathrm{E}+04$ \\
\hline $2.83 \mathrm{E}+02$ & $9.90 \mathrm{E}+03$ & $1.47 \mathrm{E}+04$ & $1.56 \mathrm{E}+04$ & $9.91 \mathrm{E}+03$ & $1.48 \mathrm{E}+04$ & $1.57 \mathrm{E}+04$ \\
\hline $1.02 \mathrm{E}+03$ & $2.56 \mathrm{E}+03$ & $4.45 \mathrm{E}+03$ & $4.87 \mathrm{E}+03$ & $2.58 \mathrm{E}+03$ & $4.52 \mathrm{E}+03$ & $4.94 \mathrm{E}+03$ \\
\hline $3.65 \mathrm{E}+03$ & $7.29 \mathrm{E}+02$ & $1.54 \mathrm{E}+03$ & $1.74 \mathrm{E}+03$ & $7.40 \mathrm{E}+02$ & $1.58 \mathrm{E}+03$ & $1.79 \mathrm{E}+03$ \\
\hline $5.48 \mathrm{E}+03$ & $6.66 \mathrm{E}+02$ & $1.35 \mathrm{E}+03$ & $1.51 \mathrm{E}+03$ & $6.74 \mathrm{E}+02$ & $1.38 \mathrm{E}+03$ & $1.55 \mathrm{E}+03$ \\
\hline $6.73 \mathrm{E}+03$ & $6.41 \mathrm{E}+02$ & $1.27 \mathrm{E}+03$ & $1.41 \mathrm{E}+03$ & $6.48 \mathrm{E}+02$ & $1.30 \mathrm{E}+03$ & $1.45 \mathrm{E}+03$ \\
\hline $8.26 \mathrm{E}+03$ & $6.15 \mathrm{E}+02$ & $1.18 \mathrm{E}+03$ & $1.31 \mathrm{E}+03$ & $6.21 \mathrm{E}+02$ & $1.21 \mathrm{E}+03$ & $1.34 \mathrm{E}+03$ \\
\hline $1.01 \mathrm{E}+04$ & $5.87 \mathrm{E}+02$ & $1.09 \mathrm{E}+03$ & $1.20 \mathrm{E}+03$ & $5.91 \mathrm{E}+02$ & $1.12 \mathrm{E}+03$ & $1.23 \mathrm{E}+03$ \\
\hline $1.24 \mathrm{E}+04$ & $5.54 \mathrm{E}+02$ & $9.95 \mathrm{E}+02$ & $1.09 \mathrm{E}+03$ & $5.58 \mathrm{E}+02$ & $1.02 \mathrm{E}+03$ & $1.11 \mathrm{E}+03$ \\
\hline $1.53 \mathrm{E}+04$ & $5.18 \mathrm{E}+02$ & $8.96 \mathrm{E}+02$ & $9.70 \mathrm{E}+02$ & $5.21 \mathrm{E}+02$ & $9.15 \mathrm{E}+02$ & $9.94 \mathrm{E}+02$ \\
\hline $1.88 \mathrm{E}+04$ & $4.79 \mathrm{E}+02$ & $7.95 \mathrm{E}+02$ & $8.52 \mathrm{E}+02$ & $4.81 \mathrm{E}+02$ & $8.12 \mathrm{E}+02$ & $8.74 \mathrm{E}+02$ \\
\hline $2.30 \mathrm{E}+04$ & $4.39 \mathrm{E}+02$ & $6.97 \mathrm{E}+02$ & $7.39 \mathrm{E}+02$ & $4.40 \mathrm{E}+02$ & $7.12 \mathrm{E}+02$ & $7.58 \mathrm{E}+02$ \\
\hline $2.83 \mathrm{E}+04$ & $3.99 \mathrm{E}+02$ & $6.06 \mathrm{E}+02$ & $6.35 \mathrm{E}+02$ & $3.99 \mathrm{E}+02$ & $6.18 \mathrm{E}+02$ & $6.51 \mathrm{E}+02$ \\
\hline $3.47 \mathrm{E}+04$ & $3.61 \mathrm{E}+02$ & $5.24 \mathrm{E}+02$ & $5.43 \mathrm{E}+02$ & $3.61 \mathrm{E}+02$ & $5.35 \mathrm{E}+02$ & $5.58 \mathrm{E}+02$ \\
\hline $3.65 \mathrm{E}+04$ & $3.52 \mathrm{E}+02$ & $5.06 \mathrm{E}+02$ & $5.23 \mathrm{E}+02$ & $3.52 \mathrm{E}+02$ & $5.17 \mathrm{E}+02$ & $5.37 \mathrm{E}+02$ \\
\hline
\end{tabular}




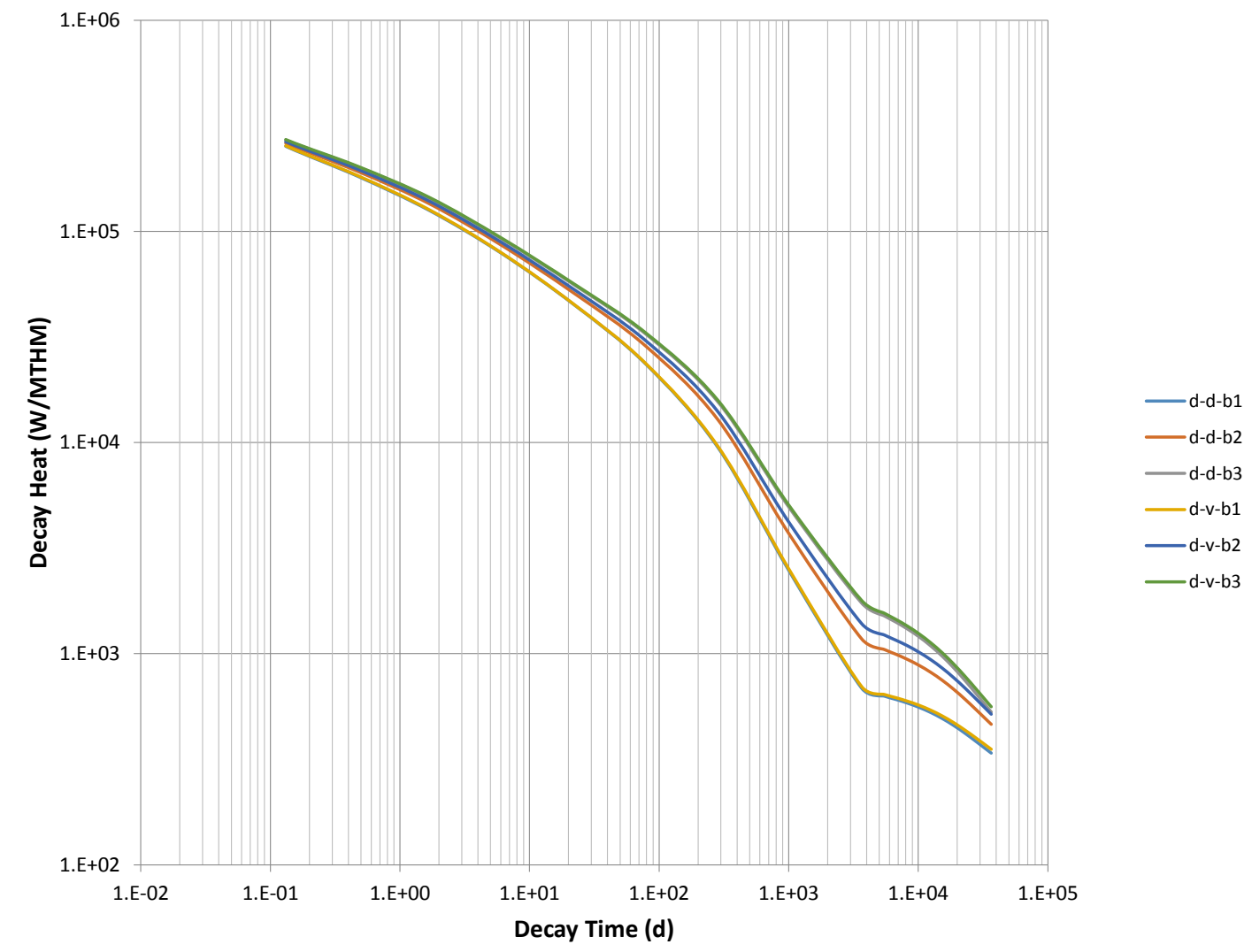

Figure 27. Comparison of "D" assembly decay heat trajectories in the BFN equilibrium MOX core - 100 years.

Table 22. Decay heat (W/MTHM) for the fuel "D" assembly in the BFN MOX core

\begin{tabular}{||c||c|c|c|c|c|c||}
\hline \multirow{2}{*}{ Time (d) } & \multicolumn{3}{|c|}{ Dominant zone } & \multicolumn{3}{c||}{ Vanished zone } \\
\cline { 2 - 7 } & $\mathbf{b 1}$ & $\mathbf{b 2}$ & $\mathbf{b 3}$ & $\mathbf{b 1}$ & $\mathbf{b 2}$ & b3 \\
\hline $0.00 \mathrm{E}+00$ & $1.68 \mathrm{E}+06$ & $1.69 \mathrm{E}+06$ & $1.71 \mathrm{E}+06$ & $1.68 \mathrm{E}+06$ & $1.71 \mathrm{E}+06$ & $1.72 \mathrm{E}+06$ \\
\hline $3.65 \mathrm{E}-02$ & $3.71 \mathrm{E}+05$ & $3.78 \mathrm{E}+05$ & $3.87 \mathrm{E}+05$ & $3.72 \mathrm{E}+05$ & $3.82 \mathrm{E}+05$ & $3.89 \mathrm{E}+05$ \\
\hline $1.31 \mathrm{E}-01$ & $2.54 \mathrm{E}+05$ & $2.62 \mathrm{E}+05$ & $2.71 \mathrm{E}+05$ & $2.54 \mathrm{E}+05$ & $2.66 \mathrm{E}+05$ & $2.72 \mathrm{E}+05$ \\
\hline $4.72 \mathrm{E}-01$ & $1.83 \mathrm{E}+05$ & $1.93 \mathrm{E}+05$ & $2.02 \mathrm{E}+05$ & $1.84 \mathrm{E}+05$ & $1.97 \mathrm{E}+05$ & $2.03 \mathrm{E}+05$ \\
\hline $1.70 \mathrm{E}+00$ & $1.26 \mathrm{E}+05$ & $1.35 \mathrm{E}+05$ & $1.44 \mathrm{E}+05$ & $1.26 \mathrm{E}+05$ & $1.39 \mathrm{E}+05$ & $1.45 \mathrm{E}+05$ \\
\hline $6.09 \mathrm{E}+00$ & $7.87 \mathrm{E}+04$ & $8.59 \mathrm{E}+04$ & $9.23 \mathrm{E}+04$ & $7.89 \mathrm{E}+04$ & $8.86 \mathrm{E}+04$ & $9.29 \mathrm{E}+04$ \\
\hline $2.19 \mathrm{E}+01$ & $4.51 \mathrm{E}+04$ & $5.11 \mathrm{E}+04$ & $5.60 \mathrm{E}+04$ & $4.52 \mathrm{E}+04$ & $5.32 \mathrm{E}+04$ & $5.65 \mathrm{E}+04$ \\
\hline $7.87 \mathrm{E}+01$ & $2.36 \mathrm{E}+04$ & $2.87 \mathrm{E}+04$ & $3.27 \mathrm{E}+04$ & $2.37 \mathrm{E}+04$ & $3.04 \mathrm{E}+04$ & $3.31 \mathrm{E}+04$ \\
\hline $2.83 \mathrm{E}+02$ & $9.52 \mathrm{E}+03$ & $1.29 \mathrm{E}+04$ & $1.57 \mathrm{E}+04$ & $9.62 \mathrm{E}+03$ & $1.40 \mathrm{E}+04$ & $1.59 \mathrm{E}+04$ \\
\hline $1.02 \mathrm{E}+03$ & $2.44 \mathrm{E}+03$ & $3.66 \mathrm{E}+03$ & $4.89 \mathrm{E}+03$ & $2.48 \mathrm{E}+03$ & $4.14 \mathrm{E}+03$ & $4.99 \mathrm{E}+03$ \\
\hline $3.65 \mathrm{E}+03$ & $6.86 \mathrm{E}+02$ & $1.18 \mathrm{E}+03$ & $1.74 \mathrm{E}+03$ & $6.98 \mathrm{E}+02$ & $1.39 \mathrm{E}+03$ & $1.79 \mathrm{E}+03$ \\
\hline $5.48 \mathrm{E}+03$ & $6.29 \mathrm{E}+02$ & $1.05 \mathrm{E}+03$ & $1.51 \mathrm{E}+03$ & $6.40 \mathrm{E}+02$ & $1.23 \mathrm{E}+03$ & $1.55 \mathrm{E}+03$ \\
\hline $6.73 \mathrm{E}+03$ & $6.06 \mathrm{E}+02$ & $9.94 \mathrm{E}+02$ & $1.41 \mathrm{E}+03$ & $6.18 \mathrm{E}+02$ & $1.16 \mathrm{E}+03$ & $1.45 \mathrm{E}+03$ \\
\hline $8.26 \mathrm{E}+03$ & $5.83 \mathrm{E}+02$ & $9.40 \mathrm{E}+02$ & $1.31 \mathrm{E}+03$ & $5.95 \mathrm{E}+02$ & $1.09 \mathrm{E}+03$ & $1.35 \mathrm{E}+03$ \\
\hline $1.01 \mathrm{E}+04$ & $5.56 \mathrm{E}+02$ & $8.81 \mathrm{E}+02$ & $1.20 \mathrm{E}+03$ & $5.69 \mathrm{E}+02$ & $1.02 \mathrm{E}+03$ & $1.24 \mathrm{E}+03$ \\
\hline $1.24 \mathrm{E}+04$ & $5.27 \mathrm{E}+02$ & $8.17 \mathrm{E}+02$ & $1.09 \mathrm{E}+03$ & $5.40 \mathrm{E}+02$ & $9.37 \mathrm{E}+02$ & $1.13 \mathrm{E}+03$ \\
\hline $1.53 \mathrm{E}+04$ & $4.93 \mathrm{E}+02$ & $7.49 \mathrm{E}+02$ & $9.74 \mathrm{E}+02$ & $5.07 \mathrm{E}+02$ & $8.54 \mathrm{E}+02$ & $1.01 \mathrm{E}+03$ \\
\hline $1.88 \mathrm{E}+04$ & $4.57 \mathrm{E}+02$ & $6.79 \mathrm{E}+02$ & $8.58 \mathrm{E}+02$ & $4.71 \mathrm{E}+02$ & $7.69 \mathrm{E}+02$ & $8.93 \mathrm{E}+02$ \\
\hline $2.30 \mathrm{E}+04$ & $4.19 \mathrm{E}+02$ & $6.08 \mathrm{E}+02$ & $7.45 \mathrm{E}+02$ & $4.33 \mathrm{E}+02$ & $6.85 \mathrm{E}+02$ & $7.79 \mathrm{E}+02$ \\
\hline $2.83 \mathrm{E}+04$ & $3.81 \mathrm{E}+02$ & $5.40 \mathrm{E}+02$ & $6.41 \mathrm{E}+02$ & $3.96 \mathrm{E}+02$ & $6.05 \mathrm{E}+02$ & $6.74 \mathrm{E}+02$ \\
\hline $3.47 \mathrm{E}+04$ & $3.46 \mathrm{E}+02$ & $4.78 \mathrm{E}+02$ & $5.50 \mathrm{E}+02$ & $3.61 \mathrm{E}+02$ & $5.33 \mathrm{E}+02$ & $5.82 \mathrm{E}+02$ \\
\hline $3.65 \mathrm{E}+04$ & $3.38 \mathrm{E}+02$ & $4.64 \mathrm{E}+02$ & $5.30 \mathrm{E}+02$ & $3.52 \mathrm{E}+02$ & $5.17 \mathrm{E}+02$ & $5.61 \mathrm{E}+02$ \\
\hline \hline
\end{tabular}




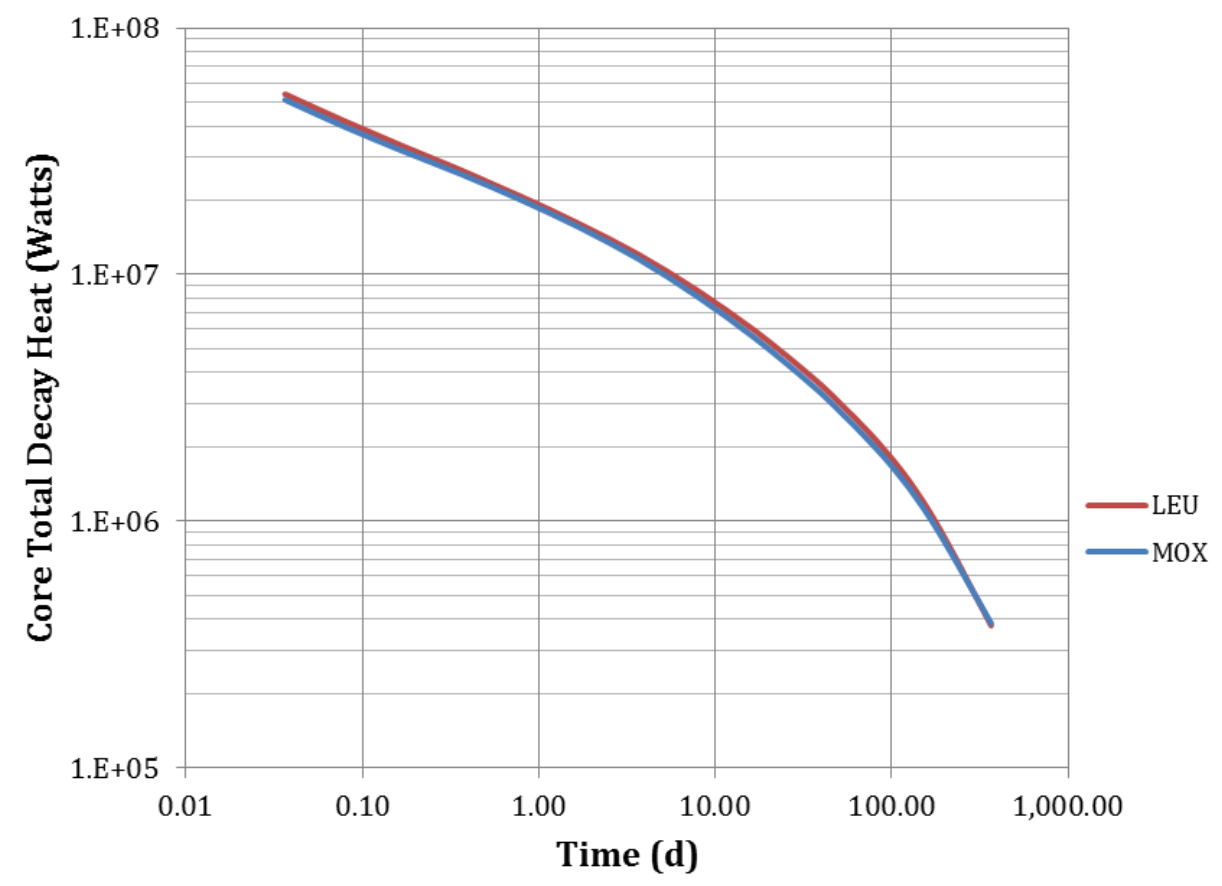

Figure 28. Comparison of decay heat in BFN equilibrium MOX and LEU cores - 1 year.

Table 23. Decay heat for the BFN equilibrium cores -1 year

\begin{tabular}{||c|c|c||c|c|c||}
\hline \hline Time (d) & LEU $(\mathbf{W})$ & MOX $(\mathbf{W})$ & Time $(d)$ & LEU (W) & MOX (W) \\
\hline \hline $0.00 \mathrm{E}+00$ & $2.57 \mathrm{E}+08$ & $2.40 \mathrm{E}+08$ & $5.48 \mathrm{E}+01$ & $2.86 \mathrm{E}+06$ & $2.64 \mathrm{E}+06$ \\
\hline $3.65 \mathrm{E}-03$ & $5.40 \mathrm{E}+07$ & $5.12 \mathrm{E}+07$ & $6.73 \mathrm{E}+01$ & $2.46 \mathrm{E}+06$ & $2.28 \mathrm{E}+06$ \\
\hline $1.02 \mathrm{E}-02$ & $4.21 \mathrm{E}+07$ & $3.99 \mathrm{E}+07$ & $8.26 \mathrm{E}+01$ & $2.11 \mathrm{E}+06$ & $1.95 \mathrm{E}+06$ \\
\hline $2.83 \mathrm{E}-02$ & $3.32 \mathrm{E}+07$ & $3.17 \mathrm{E}+07$ & $1.01 \mathrm{E}+02$ & $1.77 \mathrm{E}+06$ & $1.65 \mathrm{E}+06$ \\
\hline $7.87 \mathrm{E}-02$ & $2.65 \mathrm{E}+07$ & $2.55 \mathrm{E}+07$ & $1.24 \mathrm{E}+02$ & $1.47 \mathrm{E}+06$ & $1.37 \mathrm{E}+06$ \\
\hline $2.19 \mathrm{E}-01$ & $2.08 \mathrm{E}+07$ & $2.02 \mathrm{E}+07$ & $1.53 \mathrm{E}+02$ & $1.18 \mathrm{E}+06$ & $1.12 \mathrm{E}+06$ \\
\hline $6.09 \mathrm{E}-01$ & $1.61 \mathrm{E}+07$ & $1.56 \mathrm{E}+07$ & $1.88 \mathrm{E}+02$ & $9.25 \mathrm{E}+05$ & $8.85 \mathrm{E}+05$ \\
\hline $1.70 \mathrm{E}+00$ & $1.21 \mathrm{E}+07$ & $1.16 \mathrm{E}+07$ & $2.30 \mathrm{E}+02$ & $7.08 \mathrm{E}+05$ & $6.89 \mathrm{E}+05$ \\
\hline $4.72 \mathrm{E}+00$ & $8.64 \mathrm{E}+06$ & $8.17 \mathrm{E}+06$ & $2.83 \mathrm{E}+02$ & $5.35 \mathrm{E}+05$ & $5.32 \mathrm{E}+05$ \\
\hline $1.31 \mathrm{E}+01$ & $5.89 \mathrm{E}+06$ & $5.50 \mathrm{E}+06$ & $3.47 \mathrm{E}+02$ & $4.04 \mathrm{E}+05$ & $4.11 \mathrm{E}+05$ \\
\hline $3.65 \mathrm{E}+01$ & $3.76 \mathrm{E}+06$ & $3.49 \mathrm{E}+06$ & $3.65 \mathrm{E}+02$ & $3.77 \mathrm{E}+05$ & $3.86 \mathrm{E}+05$ \\
\hline
\end{tabular}




\section{SUMMARY}

This study has calculated

- MOX and LEU core average nuclide concentrations and core nuclide loads,

- MOX core to LEU core nuclide ratios,

- radioactivity sources, and

- decay heat curves for individual fuel assemblies and core-average decay heat values

for Sequoyah (PWR) and Browns Ferry (BWR) reactors. The SCALE/TRITON models utilized the mechanical design of the actual SQN and BFN assemblies with current proposed core (Pu-Am) assay and U-235 enrichment loadings (LEU and MOX) at SQN and BFN. The transport models used for the depletion calculations are accurate 2D representations of the proposed assembly lattices including all fuel rod assays/enrichments and locations, geometrical heterogeneities, and cross-section adjustments for individually calculated Dancoff factors in the BWR lattices. The Dancoff factors and the depletion models for the BFN cores used the average void concentration in the dominant and vanished regions of the ATRIUM 10 lattices. The fuel rod Dancoff factors automatically calculated by TRITON were deemed to be of acceptable accuracy for the SQN assemblies and were used as such in the depletion models for the SQN cores.

The SQN lattices were assumed to cover the full axial fuel length. There are no adjustments for grid spacer regions or axial blankets.

The BFN assemblies were assumed to consist of a lower dominant lattice and an upper vanished lattice, where part length rods had disappeared. In some cases, either the lower or upper region may have several lattices of slightly varying enrichment. For this study, the major lattice in the lower and upper zones was assumed to extend for the full length of that zone.

Depletion calculations for chosen lattices were carried out to $\sim 60 \mathrm{GWd} / \mathrm{MTHM}$ burnup to ensure that the burnup ranges attained within the cores were covered. For a given assembly type in a core, there were two to three burnup "classes" that could be characterized as low, medium, and high. The average burnup in each of these classes was determined for each assembly type based on the burnup distributions from the equilibrium core fuel management studies. The nuclide concentrations (g/MTHM) were interpolated at the corresponding average burnup of a burnup class of a particular fuel assembly type. The core load for each nuclide was calculated as the weighted sum of each nuclide concentration times the number of metric tonnes of heavy metals in the core. This same weighting procedure was applied to the radioactive source strengths and the core decay heat generation.

The results of the MOX/LEU concentration ratios were compared to the previously employed (PWR) ratios [3] in Table 7 (for SQN) and in Table 9 (for BFN) of the current report. For many nuclides, the differences between the newly calculated and the previously reported nuclide ratios are minimal $(<1-2 \%)$. The differences for the plutonium isotopes (7-22\%) and for the curium isotopes (18-42\%) are more substantial. These larger differences are the result of the use of more modern, up-to-date computational methods and nuclear data in the current study.

The same modeling methods and nuclear data as used in this study were also used previously to benchmark nuclide measurements in high-burnup BWR assemblies [10]; for those cases, the agreement between the experimental and calculated concentrations for the plutonium isotopes was between 0.7 and $3.4 \%$. The differences noted between the plutonium results documented in the current study and those previously reported [3] are likely due to the use of more modern, up-to-date computational methods and 
nuclear data; the current study used the more recent ENDF-B/VII cross sections as well as the sophisticated cross section self-shielding and geometry capabilities of the TRITON code system available in 2011, which did not exist in 1995.

The conspicuous difference for the nuclide Mo-92 production in the MOX and LEU cores is a direct result of the much greater fission yield of this nuclide by plutonium isotopes compared to the fission yield of this nuclide by $\mathrm{U}-235$. 


\section{REFERENCES}

1. SCALE: A Modular Code System for Performing Standardized Computer Analyses for Licensing Evaluations, ORNL/TM-2005/39, Version 6.1, May 2011.

2. Radionuclide Source Terms for an Environmental Critique of Reactor-Based Plutonium Disposition, ORNL Letter-to-file, ORNL/CF-99/57, September 1999.

3. Supplement to the Surplus Plutonium Disposition Draft Environmental Impact Statement, DOE/EIS0283-DS, April 1999.

4. Task 76 Inputs for Sequoyah 2, Cycle 19, Batch 21, AREVA NP Inc. report 51-9139812-001 (proprietary).

5. TVA Equilibrium MOX Cycle Design and Expanded PFCD Checks, AREVA NP Inc. report 129164116-001, July 2011 (proprietary).

6. SQN CGU Equilibrium Preliminary Fuel Cycle Design, AREVA NP Inc. report 12-9160713-000, September 2011 (proprietary).

7. Feasibility Study of Mixed Oxide (MOX) Fuel in BWR 24-Month Cycle with 120\% Power Uprate and Four MOX Fuel Rod Types, AREVA NP Inc. report 47-9157290-000, April 2011 (proprietary).

8. Feasibility Study of Mixed Oxide (MOX) Fuel in BWR 24-Month Cycle with 120\% Power Uprate, AREVA NP Inc. report 47-9135107-000, June 2010 (proprietary).

9. Brian Ade, NRC Cross Section Production Lessons Learned, ORNL Internal Publications, October 2010.

10. Harold J. Smith, "Modeling depletion simulations for a high-burnup, highly heterogeneous BWR fuel Assembly with SCALE," Proceedings of PHYSOR 2012, April 2012.

11. M. L. Williams, "Resonance Self Shielding Methodologies in SCALE 6," Nuclear Technology 174(2), 149 (2011). 

